\title{
Ir-Catalyzed Regio- and Enantioselective Hydroalkynylation of Tri- substituted Alkene to Access All-Carbon Quaternary Stereocenters
}

\author{
Su-Lei Zhang ${ }^{\mathrm{a}, \neq}$, Wen-Wen Zhang ${ }^{\mathrm{a}, \ddagger}$ and Bi-Jie $\mathrm{Li}^{\mathrm{a}, \mathrm{b} *}$ \\ ${ }^{\mathrm{a}}$ Center of Basic Molecular Science (CBMS), Department of Chemistry, Tsinghua University \\ ${ }^{\mathrm{b}}$ Beijing National Laboratory for Molecular Sciences
}

\section{Table of Contents}

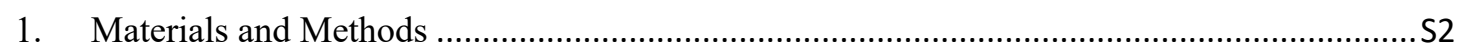

2. Optimization of Reaction Conditions and Substrate Scope Table .......................................S2

3. General Procedure for the Synthesis of Enamides.............................................................

4. General Procedure for the Synthesis of Homopropargyl Amides .........................................S5

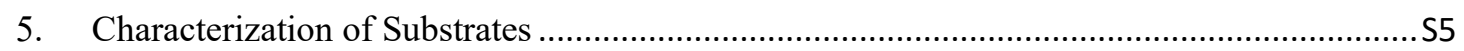

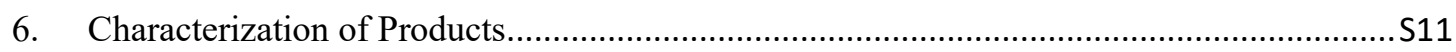

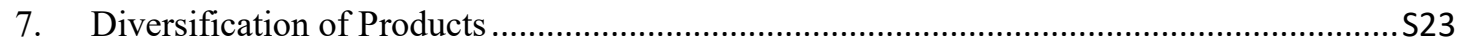

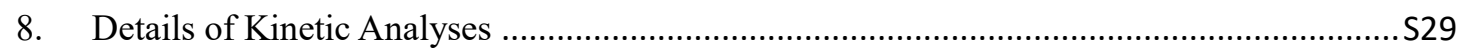

9. Control experiments for E/Z Isomerization versus Enantioselectivity ................................S31

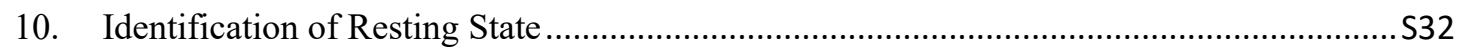

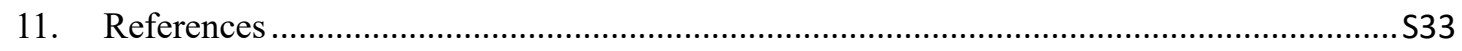

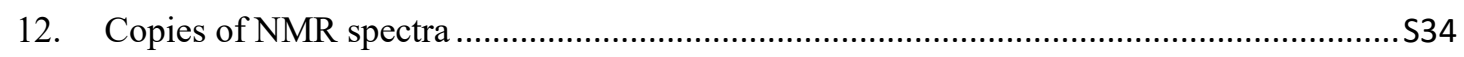

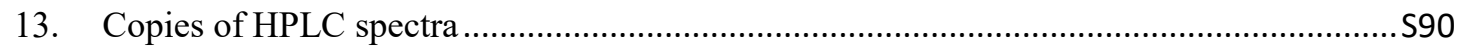




\section{Materials and Methods}

Unless otherwise noted, all reactions were assembled on a Schlenk vacuum line or in a glovebox using oven-dried glassware and were stirred with Teflon-coated magnetic stirring bars. $\left[\operatorname{Ir}(\mathrm{COD})_{2}\right] \mathrm{OTf}$ was prepared according to literature method ${ }^{1}$. $(R)-2,2^{\prime}$-Bis[bis(4-methoxy-3,5dimethylphenyl)phosphino]-4,4',6,6'-tetramethoxy)-1,1'-biphenyl $\quad$ (CAS:1365531-93-6) was purchased from Alfa Aesar and was used as received, other ligands were purchased from Strem Chemicals and were used as received. Anhydrous 1,2-Dichloroethane (DCE) were purchased from Energy Chemical and was used as received, tetrahydrofuran (THF), diethyl ether $\left(\mathrm{Et}_{2} \mathrm{O}\right)$, acetonitrile $\left(\mathrm{CH}_{3} \mathrm{CN}\right)$, dichloromethane (DCM), dimethylformamide (DMF) were degassed by purging with nitrogen and then dried with a solvent purification system containing activated alumina. All other solvents and reagents were used as purchased. All work-up and purification procedures were carried out with reagent grade solvents in air. Reaction temperatures above $23{ }^{\circ} \mathrm{C}$ refer to temperatures of an aluminum heating block or a silicon oil bath, which were controlled by an electronic temperature modulator from IKA. NMR spectra were acquired on NMR spectrometer with $400 \mathrm{MHz}$ for ${ }^{1} \mathrm{H}$ NMR and $101 \mathrm{MHz}$ for ${ }^{13} \mathrm{C}$ NMR at the NMR facility at Center of Basic Molecular Science (CBMS). Chemical shifts $(\delta)$ are reported in ppm relative to the residual solvent signal $\left(\delta=7.26\right.$ for ${ }^{1} \mathrm{H}$ NMR and $\delta=77.0$ for ${ }^{13} \mathrm{C}$ NMR). Data for ${ }^{1} \mathrm{H}$ NMR spectra are reported as follows: chemical shift (multiplicity, coupling constants, number of hydrogens). Abbreviations are as follows: s (singlet), $\mathrm{d}$ (doublet), t (triplet), q (quartet), m (multiplet), br (broad). Infrared (IR) spectra were recorded on a Bruker FT-IR alpha (ATR mode) spectrophotometer. GC data were obtained on a Shimadzu 2010 Plus GC system containing an Rxiß-5ms capillary column. High-resolution mass spectral data was performed on a Thermo Scientific Q Exactive (positive mode) at the Mass Spectrometry Facility, CBMS. Enantiomer excess (ee) values were determined by analytical liquid chromatography (HPLC) analysis on a Shimadzu chromatograph (Daicel chiral columns Chiralpak). Specific rotations were measured on a Jasco P-2000 Polari meter.

\section{Optimization of reaction conditions and substrate scope table}

Effect of various ligands was investigated in the catalytic asymmetric hydroalkynylation reactions of 1 a with 2 (Table S1). It was found that the reactions proceeded in good yields, high regioselectivity and high enantioselectivity using $\mathbf{L 9}$. 
Table S1. Reaction development in the presence of iridium catalyst

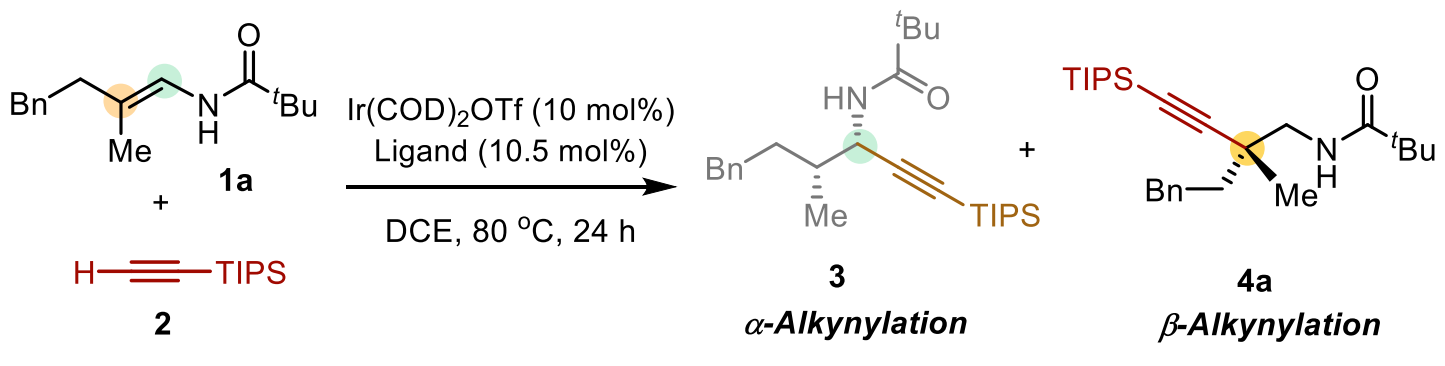<smiles>c1ccc2c(-c3c(Op4c5c(cccc6ccc7ccccc7n64)O5)ccc4ccccc34)cccc2c1</smiles>

L1

$80 \% \alpha$

$<5 \% \beta$

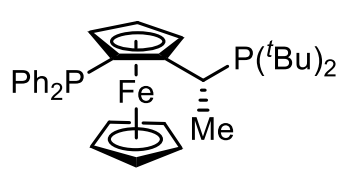

L4

$7 \% \alpha$

$7 \% \beta, 90 \%$ ee<smiles>COc1cc(OC)c(-c2c(OC)cc(OC)cc2-c2ccccc2)c(-c2ccccc2)c1</smiles>

L7
$21 \% \beta, 91 \%$ ee<smiles>CC1(C)O[C@H](CP)[C@H](CP)O1</smiles>

L2

$<5 \% \alpha$

$<5 \% \beta$<smiles>Pc1cccc(-c2ccccc2)c1-c1c(P)ccc2c1OCO2</smiles>

L5

$41 \% \alpha$ $33 \% \beta, 87 \%$ ee<smiles>COc1cc(C)cc(Pc2cc(OC)cc(OC)c2-c2c(OC)cc(OC)cc2Pc2cc(C)cc(C)c2)c1</smiles>

L8

$<5 \% \alpha$

$57 \% \beta, 93 \%$ ee

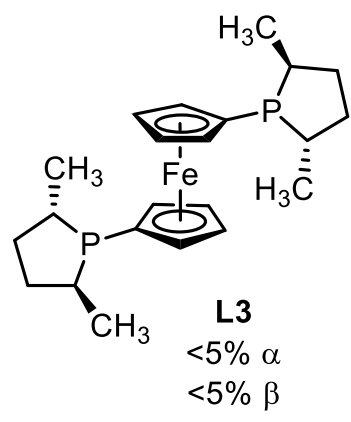<smiles>COc1cc(Pc2ccc3c(c2-c2c(Pc4cc(C(C)(C)C)c(C(C)(C)C)cc4Pc4cc(C(C)(C)C)cc(C(C)(C)C)c4)ccc4c2OCO4)OCO3)cc(CC(C)(C)C)c1OC</smiles>

L6

$<5 \%, \alpha$

$14 \% \beta, 21 \%$ ee<smiles>COc1cc(OC)c(-c2c(OC)cc(OC)cc2Pc2cc(C)c(OC)c(C)c2)c(Pc2cc(C)c(OC)c(C)c2)c1</smiles>

L9

$6 \% \alpha$

$84 \% \beta, 92 \%$ ee

${ }^{a}$ Reaction conditions: $1 \mathrm{a}(0.10 \mathrm{mmol}), 2$ (0.30 mmol), $\operatorname{Ir}(\mathrm{COD})_{2} \mathrm{OTf}(10 \mathrm{~mol} \%)$ and ligand $(10.5$ mol\%) in DCE at $80{ }^{\circ} \mathrm{C}$ for 24 hours. Yields were determined by crude ${ }^{1} \mathrm{H}$ NMR. The ee values were determined by chiral HPLC. DCE, 1,2-dichloroethane. 
Table S2. Scope of Ir-Catalyzed Hydroalkynylation of Enamides.

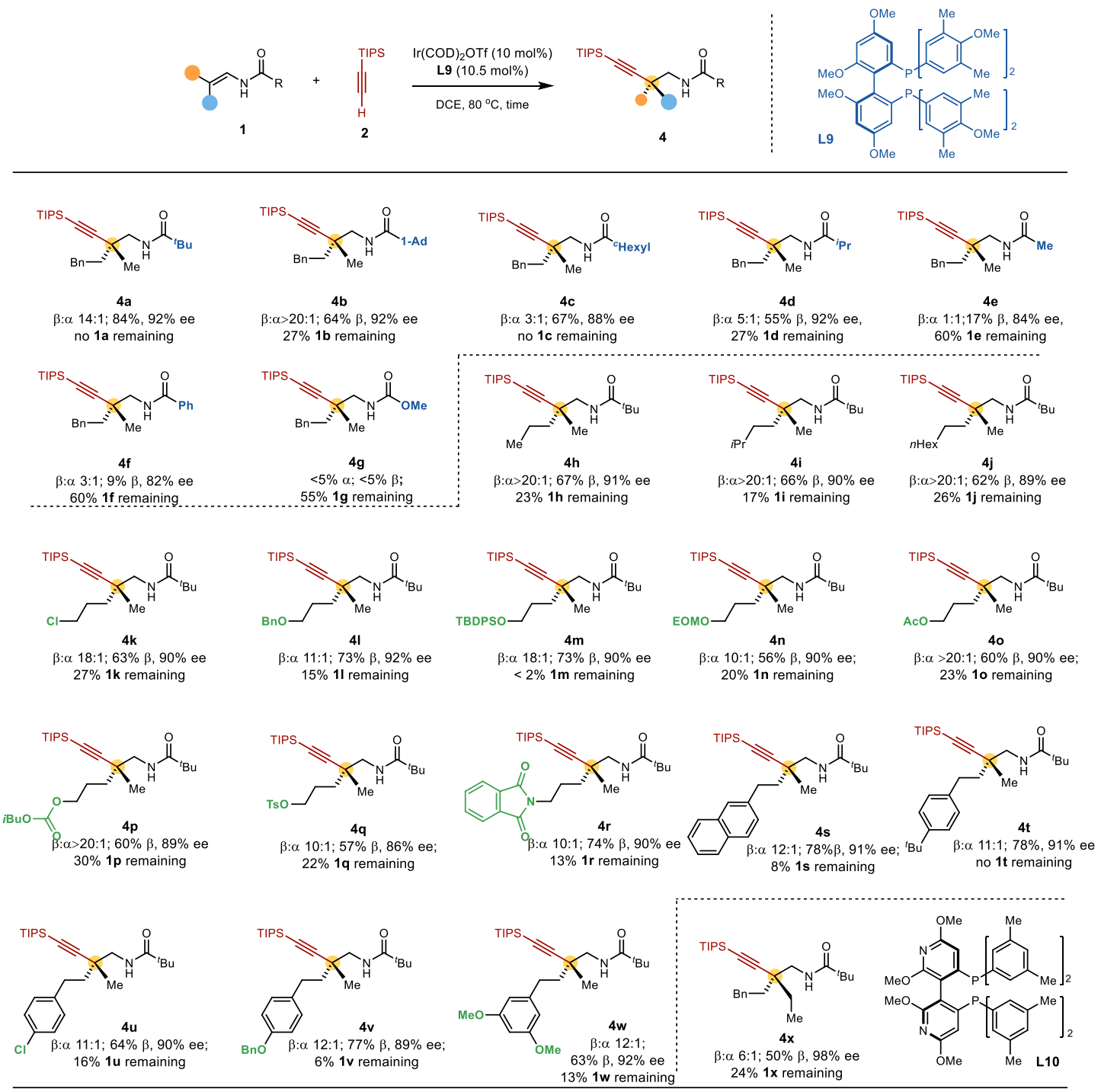

${ }^{a}$ Reaction conditions: $1 \mathbf{a}(0.10 \mathrm{mmol}), \mathbf{2}(0.30 \mathrm{mmol}), \operatorname{Ir}(\mathrm{COD})_{2} \mathrm{OTf}(10 \mathrm{~mol} \%)$ and $\mathbf{L} \mathbf{9}(10.5 \mathrm{~mol} \%)$ in DCE at $80{ }^{\circ} \mathrm{C}$ for 24 hours. The values of $\alpha: \beta$ were determined by crude ${ }^{1} \mathrm{H}$ NMR using 1,3,5-trimethoxybenzene as an internal standard. Isolated yields were reported. The ee values were determined by chiral HPLC.

\section{General Procedure for the Synthesis of Enamides}

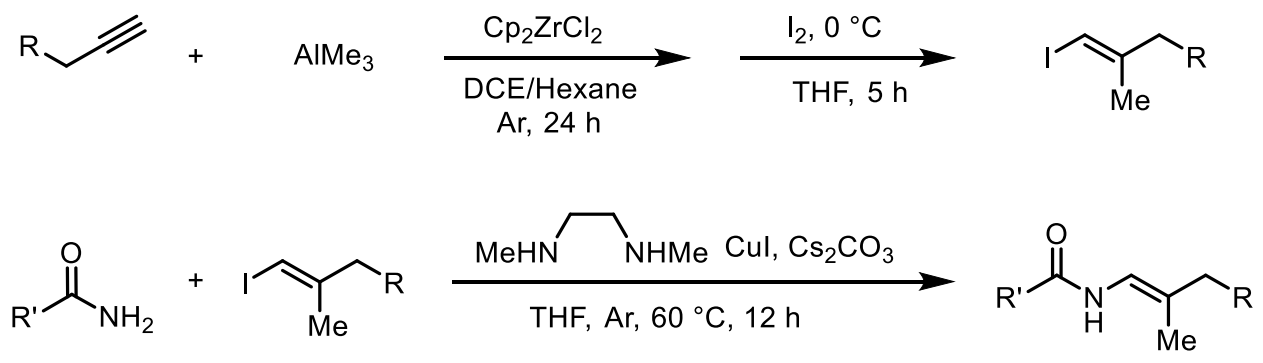

The preparation of substrates was the same as our previous report ${ }^{2}$. $\mathrm{AlMe}_{3}(2.0 \mathrm{M}$ solution in hexane, $20 \mathrm{mmol}$, 2.0 equiv) was added dropwise to a solution of $\mathrm{Cp}_{2} \mathrm{ZrCl}_{2}(10 \mathrm{mmol}, 1.0$ equiv $)$ in DCE $(15 \mathrm{~mL})$ at $0{ }^{\circ} \mathrm{C}^{3}$. After stirring for $1.0 \mathrm{~h}$, alkyne $\left(10 \mathrm{mmol}, 1.0\right.$ equiv) was added at $0{ }^{\circ} \mathrm{C}$ and 
the reaction mixture was stirred for $24 \mathrm{~h}$ at room temperature. The reaction mixture was then cooled to $0{ }^{\circ} \mathrm{C}$ and $\mathrm{I}_{2}(12 \mathrm{mmol}, 1.2$ equiv $)$ in $\mathrm{THF}(15 \mathrm{~mL})$ was added. After $5 \mathrm{~h}$, the reaction mixture was quenched with $\mathrm{H}_{2} \mathrm{O}$ carefully at $0{ }^{\circ} \mathrm{C}$. The organic layer was extracted with ether, washed with $\mathrm{Na}_{2} \mathrm{~S}_{2} \mathrm{O}_{3}$, dried with $\mathrm{Na}_{2} \mathrm{SO}_{4}$, concentrated in vacuo. The crude product was purified by flash chromatography on silica gel with petroleum ether as an eluent, and vinyl iodides was obtained as a single isomer.

A Schlenk tube charged with amide ${ }^{4}(20 \mathrm{mmol}, 2.0$ equiv), copper(I) iodide ( $20 \mathrm{mmol}, 2.0$ equiv), and $\mathrm{Cs}_{2} \mathrm{CO}_{3}$ (20 mmol, 2.0 equiv) was evacuated under high vacuum, backfilled with argon, and closed with a rubber septum. Vinyl iodide (10 mmol, 1.0 equiv) and $N, N^{\prime}$ dimethylethylenediamine ( $20 \mathrm{mmol}, 2.0$ equiv) in THF were added. The mixture was stirred at $60^{\circ} \mathrm{C}$ for $12 \mathrm{~h}$. After filtration over celite, the mixture was diluted with DCM and washed with water. The organic extract was dried over $\mathrm{Na}_{2} \mathrm{SO}_{4}$ and concentrated in vacuo. The crude product was purified by flash chromatography on silica gel with PE/EtOAc mixture as eluent.

\section{General Procedure for the Synthesis of Homopropargyl Amides}

In an $\mathrm{N}_{2}$-filled glovebox, the enamide ( $0.10 \mathrm{mmol}, 1.0$ equiv), $\operatorname{Ir}(\mathrm{COD})_{2} \mathrm{OTf}(5.6 \mathrm{mg}, 0.010$ mmol), $(R)$-Ligand (9.2 $\mathrm{mg}, 0.0105 \mathrm{mmol})$ was weighed into a screw-capped vial. Subsequently, DCE $(0.30 \mathrm{~mL})$ and alkyne $(0.30 \mathrm{mmol}, 3.0$ equiv) were added via syringes. The vial was capped with a Teflon-lined screw cap, and the resulting solution was then removed from the glovebox, placed in an aluminum block and heated to $40{ }^{\circ} \mathrm{C}$ for $1 \mathrm{~h}$ then $80{ }^{\circ} \mathrm{C}$ for $24 \mathrm{~h}$ or $48 \mathrm{~h}$. The reaction mixture was purified directly by column chromatography on silica gel with PE/EtOAc mixture as eluent.

\section{Characterization of Substrates}<smiles>[R]C(=O)N/C=C(\C)CBr</smiles>

$$
\mathrm{R}=\mathrm{Ph}, \mathrm{Me},{ }^{i} \mathrm{Pr},{ }^{t} \mathrm{Bu}
$$

Enamide 1a, 1d, 1e, 1f were the same as the previous report ${ }^{2}$.<smiles>COC(=O)N/C=C(\C)CBr</smiles>

Enamide 1b: ${ }^{1} \mathbf{H}$ NMR $\left(400 \mathrm{MHz}, \mathrm{CDCl}_{3}\right) \delta 7.31-7.26(\mathrm{~m}, 2 \mathrm{H}), 7.21-7.15(\mathrm{~m}, 3 \mathrm{H}), 6.96(\mathrm{~d}, J=$ 
$10.4 \mathrm{~Hz}, 1 \mathrm{H}), 6.65(\mathrm{dq}, J=10.4,1.4 \mathrm{~Hz}, 1 \mathrm{H}), 2.76-2.67(\mathrm{~m}, 2 \mathrm{H}), 2.38-2.26(\mathrm{~m}, 2 \mathrm{H}), 2.07(\mathrm{~s}$, 3H), $1.89(\mathrm{~d}, J=2.9 \mathrm{~Hz}, 6 \mathrm{H}), 1.80-1.69(\mathrm{~m}, 6 \mathrm{H}), 1.67(\mathrm{~d}, J=1.4 \mathrm{~Hz}, 3 \mathrm{H}) .{ }^{13} \mathbf{C} \mathbf{N M R}(101 \mathrm{MHz}$, $\left.\mathrm{CDCl}_{3}\right) \delta 174.7,141.9,128.4,128.3,125.9,117.9,117.7,40.8,39.2,38.8,36.5,34.9,28.1,14.9$.

ESI-HR calcd for $\mathrm{C}_{22} \mathrm{H}_{30} \mathrm{NO}^{+}\left([\mathrm{M}+\mathrm{H}]^{+}\right) 324.2322$, found 342.2313. IR $v\left(\mathrm{~cm}^{-1}\right) 3465,2908,2852$, 2361, 1734, 1493, 1264, 739, 702. M.P. $95-96^{\circ} \mathrm{C}$.<smiles>CC(=CNC(=O)O)CBr</smiles>

Enamide 1c: ${ }^{1} \mathrm{H}$ NMR $\left(400 \mathrm{MHz}, \mathrm{CDCl}_{3}\right) \delta 7.31-7.26(\mathrm{~m}, 2 \mathrm{H}), 7.17(\mathrm{~d}, J=7.5 \mathrm{~Hz}, 3 \mathrm{H}), 6.73(\mathrm{~s}$, 1H), $6.63(\mathrm{~d}, J=10.4 \mathrm{~Hz}, 1 \mathrm{H}), 2.79-2.64(\mathrm{~m}, 2 \mathrm{H}), 2.39-2.25(\mathrm{~m}, 2 \mathrm{H}), 2.13(\mathrm{tt}, J=11.7,3.6 \mathrm{~Hz}$, $1 \mathrm{H}), 1.89(\mathrm{~d}, J=12.8 \mathrm{~Hz}, 2 \mathrm{H}), 1.81(\mathrm{~d}, J=11.9 \mathrm{~Hz}, 2 \mathrm{H}), 1.73-1.62(\mathrm{~m}, 4 \mathrm{H}), 1.47(\mathrm{q}, J=12.0 \mathrm{~Hz}$, 2H), $1.37-1.18(\mathrm{~m}, 3 \mathrm{H}) .{ }^{13} \mathrm{C}$ NMR $\left(101 \mathrm{MHz}, \mathrm{CDCl}_{3}\right) \delta 172.9,141.9,128.4,128.3,125.9,117.7$, 117.6, 45.6, 38.7, 34.8, 29.7, 25.7, 14.9. ESI-HR calcd for $\mathrm{C}_{18} \mathrm{H}_{26} \mathrm{NO}^{+}\left([\mathrm{M}+\mathrm{H}]^{+}\right)$272.2009, found 272.2000. IR $v\left(\mathrm{~cm}^{-1}\right)$ 3298, 2931, 2854, 1639, 1517, 1453, 1253, 1213, 956, 847, 693. M.P. 126 $127^{\circ} \mathrm{C}$.<smiles>COC(=O)N/C=C(\C)CBr</smiles>

Enamide 1g: ${ }^{1} \mathbf{H}$ NMR $\left(400 \mathrm{MHz}, \mathrm{CDCl}_{3}\right) \delta 7.27(\mathrm{~d}, J=7.1 \mathrm{~Hz}, 2 \mathrm{H}), 7.22-7.13(\mathrm{~m}, 3 \mathrm{H}), 6.32(\mathrm{~d}$, $J=10.6 \mathrm{~Hz}, 1 \mathrm{H}), 6.10$ (d, $J=9.1 \mathrm{~Hz}, 1 \mathrm{H}), 3.72(\mathrm{~s}, 3 \mathrm{H}), 2.77-2.64(\mathrm{~m}, 2 \mathrm{H}), 2.34-2.23(\mathrm{~m}, 2 \mathrm{H})$, $1.61(\mathrm{~d}, J=1.4 \mathrm{~Hz}, 3 \mathrm{H}) .{ }^{13} \mathbf{C}$ NMR $\left(101 \mathrm{MHz}, \mathrm{CDCl}_{3}\right) \delta 154.2,141.8,128.3,125.8,118.5,115.8$, 52.3, 38.5, 34.8, 14.5. ESI-HR calcd for $\mathrm{C}_{13} \mathrm{H}_{18} \mathrm{NO}_{2}{ }^{+}\left([\mathrm{M}+\mathrm{H}]^{+}\right)$220.1332, found 220.1327. IR v $\left(\mathrm{cm}^{-1}\right) 3033,2952,1498,1454,1265,737,700$.<smiles>CCCCC(=O)N/C=C(\CC)CCC</smiles>

Enamide 1h: ${ }^{1} \mathbf{H}$ NMR $\left(400 \mathrm{MHz}, \mathrm{CDCl}_{3}\right) \delta 6.97(\mathrm{~s}, 1 \mathrm{H}), 6.56(\mathrm{dq}, J=10.2,1.3 \mathrm{~Hz}, 1 \mathrm{H}), 1.99(\mathrm{t}$, $J=7.5 \mathrm{~Hz}, 2 \mathrm{H}), 1.60$ (d, $J=1.5 \mathrm{~Hz}, 3 \mathrm{H}), 1.43$ (h, $J=7.3 \mathrm{~Hz}, 2 \mathrm{H}), 1.24$ (s, 9H), 0.88 (t, $J=7.3 \mathrm{~Hz}$, 3H). ${ }^{13} \mathrm{C}$ NMR (101 MHz, $\left.\mathrm{CDCl}_{3}\right) \delta 175.1,118.5,117.4,77.4,77.0,76.7,38.8,38.7,27.5,21.0$, 14.5, 13.7. ESI-HR calcd for $\mathrm{C}_{11} \mathrm{H}_{22} \mathrm{NO}^{+}\left([\mathrm{M}+\mathrm{H}]^{+}\right)$184.1696, found 184.1691. IR $v\left(\mathrm{~cm}^{-1}\right) 2959$, 2930, 1648, 1493, 1265, 1195, 734, 703. M.P. 57 - $58^{\circ} \mathrm{C}$. 
<smiles>CCCCC(=O)N/C=C(\C)CCC(C)C</smiles>

Enamide 1i: ${ }^{1} \mathbf{H}$ NMR $\left(400 \mathrm{MHz}, \mathrm{CDCl}_{3}\right) \delta 6.97(\mathrm{~s}, 1 \mathrm{H}), 6.58(\mathrm{dq}, J=10.2,1.4 \mathrm{~Hz}, 1 \mathrm{H}), 2.02(\mathrm{t}, J$ $=7.4 \mathrm{~Hz}, 2 \mathrm{H}), 1.65-1.60(\mathrm{~m}, 3 \mathrm{H}), 1.59-1.47(\mathrm{~m}, 1 \mathrm{H}), 1.34-1.28(\mathrm{~m}, 2 \mathrm{H}), 1.26(\mathrm{~s}, 9 \mathrm{H}), 0.90(\mathrm{~d}$, $J=6.6 \mathrm{~Hz}, 6 \mathrm{H}) .{ }^{13} \mathbf{C} \mathbf{N M R}\left(101 \mathrm{MHz}, \mathrm{CDCl}_{3}\right) \delta 175.1,119.0,117.1,38.8,37.2,34.5,27.6,27.5$, 22.5, 14.6. ESI-HR calcd for $\mathrm{C}_{13} \mathrm{H}_{26} \mathrm{NO}^{+}\left([\mathrm{M}+\mathrm{H}]^{+}\right)$212.2009, found 212.2003. IR $v\left(\mathrm{~cm}^{-1}\right) 2956$, 2927, 1660, 1492, 1264, 733, 703.<smiles>CC(=CNC(=O)Br)CC[14CH3]</smiles>

Enamide 1j: ${ }^{1} \mathbf{H}$ NMR (400 MHz, $\left.\mathrm{CDCl}_{3}\right) \delta 6.95(\mathrm{~s}, 1 \mathrm{H}), 6.56(\mathrm{dq}, J=10.3,1.5 \mathrm{~Hz}, 1 \mathrm{H}), 2.00(\mathrm{t}, J$ $=6.7 \mathrm{~Hz}, 2 \mathrm{H}), 1.64-1.58(\mathrm{~m}, 3 \mathrm{H}), 1.47-1.34(\mathrm{~m}, 2 \mathrm{H}), 1.33-1.18(\mathrm{~m}, 19 \mathrm{H}), 0.88(\mathrm{t}, J=6.7 \mathrm{~Hz}$, 3H). ${ }^{13} \mathbf{C}$ NMR (101 MHz, $\left.\mathrm{CDCl}_{3}\right) \delta 175.1,118.8,117.2,38.8,36.6,31.9,29.5,29.3,27.9,27.5$, 22.7, 14.6, 14.1. ESI-HR calcd for $\mathrm{C}_{16} \mathrm{H}_{32} \mathrm{NO}^{+}\left([\mathrm{M}+\mathrm{H}]^{+}\right) 254.2478$, found 254.2468. IR $v\left(\mathrm{~cm}^{-1}\right)$ 2924, 1657, 1493, 1264, 734, 704.<smiles>CC(=CNC(=O)Br)CCCCl</smiles>

Enamide 1k: ${ }^{1} \mathbf{H}$ NMR $\left(400 \mathrm{MHz}, \mathrm{CDCl}_{3}\right) \delta 7.01(\mathrm{~s}, 1 \mathrm{H}), 6.61(\mathrm{dq}, J=10.3,1.4 \mathrm{~Hz}, 1 \mathrm{H}), 3.51$ (t, $J=6.6 \mathrm{~Hz}, 2 \mathrm{H}), 2.17(\mathrm{t}, J=6.6 \mathrm{~Hz}, 2 \mathrm{H}), 1.96-1.81(\mathrm{~m}, 2 \mathrm{H}), 1.63(\mathrm{~d}, J=1.5 \mathrm{~Hz}, 3 \mathrm{H}), 1.25(\mathrm{~s}, 9 \mathrm{H})$. ${ }^{13} \mathrm{C}$ NMR $\left(101 \mathrm{MHz}, \mathrm{CDCl}_{3}\right) \delta 175.2,118.3,116.6,44.4,38.8,33.8,30.8,27.5,14.5$. ESI-HR calcd for $\mathrm{C}_{11} \mathrm{H}_{21} \mathrm{ClNO}^{+}\left([\mathrm{M}+\mathrm{H}]^{+}\right)$218.1306, found 218.1297. IR $v\left(\mathrm{~cm}^{-1}\right) 3469,3340,2958,1647$, 1492, 1198, 653, 542. M.P. $65-66^{\circ} \mathrm{C}$.<smiles>CCCCCC(C)=CNC(=O)CCCOCc1ccccc1</smiles>

Enamide 1I: ${ }^{1} \mathrm{H}$ NMR $\left(400 \mathrm{MHz}, \mathrm{CDCl}_{3}\right) \delta 7.34(\mathrm{~d}, J=3.7 \mathrm{~Hz}, 4 \mathrm{H}), 7.31-7.26(\mathrm{~m}, 1 \mathrm{H}), 6.96(\mathrm{~d}$, $J=10.3 \mathrm{~Hz}, 1 \mathrm{H}), 6.59$ (dq, $J=10.4,1.4 \mathrm{~Hz}, 1 \mathrm{H}), 4.50(\mathrm{~s}, 2 \mathrm{H}), 3.46$ (t, $J=6.5 \mathrm{~Hz}, 2 \mathrm{H}), 2.15-2.06$ (m, 2H), $1.79-1.69(\mathrm{~m}, 2 \mathrm{H}), 1.62(\mathrm{~d}, J=1.4 \mathrm{~Hz}, 3 \mathrm{H}), 1.24$ (s, 9H). ${ }^{13} \mathbf{C} \mathbf{~ N M R}\left(101 \mathrm{MHz}, \mathrm{CDCl}_{3}\right)$ $\delta 175.1,138.6,128.3,127.6,127.5,117.9,117.6,72.9,69.9,38.8,33.2,28.0,27.5,14.5$. ESI-HR 
calcd for $\mathrm{C}_{18} \mathrm{H}_{28} \mathrm{NO}_{2}^{+}\left([\mathrm{M}+\mathrm{H}]^{+}\right)$290.2115, found 290.2105. IR $v\left(\mathrm{~cm}^{-1}\right) 3469,3351,2957,2867$, 1652, 1494, 1454, 1197, 1102, 735, 698.<smiles>CCCCOCCC/C(C)=C/NC(=O)CCCCCCC(C)C</smiles>

Enamide 1 m: ${ }^{1} \mathrm{H}$ NMR $\left(400 \mathrm{MHz}, \mathrm{CDCl}_{3}\right) \delta 7.72-7.65(\mathrm{~m}, 4 \mathrm{H}), 7.40(\mathrm{tddd}, J=8.6,6.7,2.8,1.5$ $\mathrm{Hz}, 6 \mathrm{H}), 6.97$ (d, $J=10.3 \mathrm{~Hz}, 1 \mathrm{H}), 6.60(\mathrm{dq}, J=10.3,1.4 \mathrm{~Hz}, 1 \mathrm{H}), 3.67$ (t, $J=6.3 \mathrm{~Hz}, 2 \mathrm{H}), 2.17-$ $2.07(\mathrm{~m}, 2 \mathrm{H}), 1.74-1.64(\mathrm{~m}, 2 \mathrm{H}), 1.60(\mathrm{~d}, J=1.4 \mathrm{~Hz}, 3 \mathrm{H}), 1.26(\mathrm{~s}, 9 \mathrm{H}), 1.07$ (s, 9H). ${ }^{13} \mathbf{C}$ NMR $\left(101 \mathrm{MHz}, \mathrm{CDCl}_{3}\right) \delta 175.0,135.5,133.9,129.5,127.6,118.2,117.4,63.4,38.8,33.0,30.9,27.5$, 26.8, 19.2, 14.6. ESI-HR calcd for $\mathrm{C}_{27} \mathrm{H}_{40} \mathrm{NO}_{2} \mathrm{Si}^{+}\left([\mathrm{M}+\mathrm{H}]^{+}\right) 438.2823$, found 438.2814. IR $v\left(\mathrm{~cm}^{-}\right.$ 1) $3472,3352,2958,2932,2858,1662,1491,1109,738,702,505$. M.P. $67-68^{\circ} \mathrm{C}$.<smiles>CCOCOCCC/C(C)=C/NC(=O)CC(C)(C)C</smiles>

Enamide 1n: ${ }^{1} \mathbf{H}$ NMR $\left(400 \mathrm{MHz}, \mathrm{CDCl}_{3}\right) \delta 6.96(\mathrm{~d}, J=10.2 \mathrm{~Hz}, 1 \mathrm{H}), 6.58(\mathrm{dq}, J=10.3,1.5 \mathrm{~Hz}$, 1H), $4.65(\mathrm{~s}, 2 \mathrm{H}), 3.59$ (q, $J=7.1 \mathrm{~Hz}, 2 \mathrm{H}), 3.51(\mathrm{t}, J=6.5 \mathrm{~Hz}, 2 \mathrm{H}), 2.14-2.05(\mathrm{~m}, 2 \mathrm{H}), 1.75-1.65$ $(\mathrm{m}, 2 \mathrm{H}), 1.62(\mathrm{~d}, J=1.5 \mathrm{~Hz}, 3 \mathrm{H}), 1.23(\mathrm{~s}, 9 \mathrm{H}), 1.21(\mathrm{t}, J=7.1 \mathrm{~Hz}, 3 \mathrm{H}) .{ }^{13} \mathbf{C} \mathbf{N M R}\left(101 \mathrm{MHz}, \mathrm{CDCl}_{3}\right)$ $\delta$ 175.1, 117.7, 117.7, 95.1, 67.2, 63.1, 38.8, 33.2, 28.0, 27.5, 15.1, 14.5. ESI-HR calcd for $\mathrm{C}_{14} \mathrm{H}_{28} \mathrm{NO}_{3}{ }^{+}\left([\mathrm{M}+\mathrm{H}]^{+}\right)$258.2064, found 258.2057. IR $v\left(\mathrm{~cm}^{-1}\right) 3471,3351,2934,2873,1659,1492$, $1265,1195,1114,1038,733,702$.<smiles>CC(=O)OCCC/C(C)=C/NC(=O)CC(C)C</smiles>

Enamide 1o: ${ }^{1} \mathrm{H}$ NMR $\left(400 \mathrm{MHz}, \mathrm{CDCl}_{3}\right) \delta 6.97(\mathrm{~d}, J=10.3 \mathrm{~Hz}, 1 \mathrm{H}), 6.57(\mathrm{dq}, J=10.3,1.4 \mathrm{~Hz}$, $1 \mathrm{H}), 4.02(\mathrm{t}, J=6.6 \mathrm{~Hz}, 2 \mathrm{H}), 2.11-2.04(\mathrm{~m}, 2 \mathrm{H}), 2.03(\mathrm{~s}, 3 \mathrm{H}), 1.73(\mathrm{dq}, J=9.3,6.7 \mathrm{~Hz}, 2 \mathrm{H}), 1.61$ $(\mathrm{d}, J=1.3 \mathrm{~Hz}, 3 \mathrm{H}), 1.22(\mathrm{~s}, 9 \mathrm{H}) .{ }^{13} \mathbf{C}$ NMR $\left(101 \mathrm{MHz}, \mathrm{CDCl}_{3}\right) \delta 175.1,171.1,118.0,117.0,63.8$, 38.8, 32.8, 27.4, 26.8, 20.9, 14.4. ESI-HR calcd for $\mathrm{C}_{13} \mathrm{H}_{24} \mathrm{NO}_{3}{ }^{+}\left([\mathrm{M}+\mathrm{H}]^{+}\right)$242.1751, found 242.1742. IR $v\left(\mathrm{~cm}^{-1}\right) 3351,2958,1737,1650,1494,1366,1232,1195,1041,606$.<smiles>CCC(C)C(=O)N/C=C(\C)CCCOC(=O)OC(C)(C)C</smiles> 
Enamide 1p: ${ }^{1} \mathbf{H}$ NMR $\left(400 \mathrm{MHz}, \mathrm{CDCl}_{3}\right) \delta 6.97(\mathrm{~s}, 1 \mathrm{H}), 6.58(\mathrm{~d}, J=10.3 \mathrm{~Hz}, 1 \mathrm{H}), 4.09(\mathrm{t}, J=6.6$ $\mathrm{Hz}, 2 \mathrm{H}), 3.89(\mathrm{~d}, J=6.7 \mathrm{~Hz}, 2 \mathrm{H}), 2.10(\mathrm{t}, J=7.6 \mathrm{~Hz}, 2 \mathrm{H}), 2.02-1.90(\mathrm{~m}, 1 \mathrm{H}), 1.78(\mathrm{p}, J=6.9 \mathrm{~Hz}$, 2H), $1.61(\mathrm{~s}, 3 \mathrm{H}), 1.22(\mathrm{~s}, 9 \mathrm{H}), 0.93(\mathrm{~d}, J=6.7 \mathrm{~Hz}, 6 \mathrm{H}) .{ }^{13} \mathbf{C}$ NMR $\left(101 \mathrm{MHz}, \mathrm{CDCl}_{3}\right) \delta$ 175.1, 155.4, 118.0, 116.9, 74.0, 67.3, 38.8, 32.7, 27.8, 27.5, 26.9, 18.9, 14.4. ESI-HR calcd for $\mathrm{C}_{16} \mathrm{H}_{30} \mathrm{NO}_{4}{ }^{+}\left([\mathrm{M}+\mathrm{H}]^{+}\right)$300.2169, found 300.2155. IR $v\left(\mathrm{~cm}^{-1}\right) 3469,2962,1741,1663,1491,1263$, $1195,733,703$.<smiles>CC(=CNC(=O)CCCC(C)(C)C)CCCC(C)(C)C</smiles>

Enamide 1q: ${ }^{1} \mathbf{H}$ NMR $\left(400 \mathrm{MHz}, \mathrm{CDCl}_{3}\right) \delta 7.78(\mathrm{~d}, \mathrm{~J}=8.1 \mathrm{~Hz}, 2 \mathrm{H}), 7.35(\mathrm{~d}, \mathrm{~J}=8.0 \mathrm{~Hz}, 2 \mathrm{H}), 6.94$ $(\mathrm{d}, \mathrm{J}=10.3 \mathrm{~Hz}, 1 \mathrm{H}), 6.55-6.44(\mathrm{~m}, 1 \mathrm{H}), 4.00(\mathrm{t}, \mathrm{J}=6.4 \mathrm{~Hz}, 2 \mathrm{H}), 2.45(\mathrm{~s}, 3 \mathrm{H}), 2.03(\mathrm{t}, \mathrm{J}=7.6 \mathrm{~Hz}$, 2H), $1.83-1.70(\mathrm{~m}, 2 \mathrm{H}), 1.57(\mathrm{~d}, \mathrm{~J}=1.4 \mathrm{~Hz}, 3 \mathrm{H}), 1.23(\mathrm{~s}, 9 \mathrm{H}) .{ }^{13} \mathbf{C ~ N M R}\left(101 \mathrm{MHz}, \mathrm{CDCl}_{3}\right) \delta$ 175.1, 144.7, 133.0, 129.8, 127.8, 118.2, 116.3, 70.0, 38.8, 32.4, 27.4, 27.2, 21.6, 14.4. ESI-HR calcd for $\mathrm{C}_{18} \mathrm{H}_{28} \mathrm{NO}_{4} \mathrm{~S}^{+}\left([\mathrm{M}+\mathrm{H}]^{+}\right)$354.1734, found 354.1723. IR $v\left(\mathrm{~cm}^{-1}\right)$ 2962, 1662, 1494, 1357 , $1189,1176,925,737,555$.<smiles>CC(=CNC(=O)C(C)(C)C)CCCN1C(=O)c2ccccc2C1=O</smiles>

Enamide 1r: ${ }^{1} \mathrm{H}$ NMR $\left(400 \mathrm{MHz}, \mathrm{CDCl}_{3}\right) \delta 7.82(\mathrm{dd}, J=5.4,3.1 \mathrm{~Hz}, 2 \mathrm{H}), 7.77-7.62(\mathrm{~m}, 2 \mathrm{H})$, $6.96(\mathrm{~d}, J=10.4 \mathrm{~Hz}, 1 \mathrm{H}), 6.59$ (dq, $J=10.3,1.3 \mathrm{~Hz}, 1 \mathrm{H}), 3.71-3.58(\mathrm{~m}, 2 \mathrm{H}), 2.08$ (t, $J=7.6 \mathrm{~Hz}$, 2H), $1.86-1.70(\mathrm{~m}, 2 \mathrm{H}), 1.62(\mathrm{~d}, J=1.4 \mathrm{~Hz}, 3 \mathrm{H}), 1.22(\mathrm{~s}, 9 \mathrm{H}) .{ }^{13} \mathbf{C}$ NMR $\left(101 \mathrm{MHz}, \mathrm{CDCl}_{3}\right) \delta$ 175.0, 168.3, 133.8, 132.1, 123.1, 118.0, 116.8, 38.8, 37.6, 33.9, 27.4, 26.6, 14.3.ESI-HR calcd for $\mathrm{C}_{19} \mathrm{H}_{25} \mathrm{~N}_{2} \mathrm{O}_{3}{ }^{+}\left([\mathrm{M}+\mathrm{H}]^{+}\right) 329.1860$, found 329.1849. IR $v\left(\mathrm{~cm}^{-1}\right) 3468,2957,2871,1708,1661,1493$, 1396, 1037, 720, 530. M.P. 114 - $115^{\circ} \mathrm{C}$.<smiles>CC(=CNC(=O)C(C)(C)C)CCc1ccc2ccccc2c1</smiles>

Enamide 1s: ${ }^{1} \mathbf{H}$ NMR $\left(400 \mathrm{MHz}, \mathrm{CDCl}_{3}\right) \delta 7.88-7.70(\mathrm{~m}, 3 \mathrm{H}), 7.68-7.57(\mathrm{~m}, 1 \mathrm{H}), 7.50-7.38$ (m, 2H), $7.34(\mathrm{dd}, J=8.4,1.8 \mathrm{~Hz}, 1 \mathrm{H}), 6.99(\mathrm{~d}, J=10.2 \mathrm{~Hz}, 1 \mathrm{H}), 6.68(\mathrm{dq}, J=10.3,1.4 \mathrm{~Hz}, 1 \mathrm{H})$, $2.95-2.83(\mathrm{~m}, 2 \mathrm{H}), 2.48-2.37(\mathrm{~m}, 2 \mathrm{H}), 1.70(\mathrm{~d}, J=1.4 \mathrm{~Hz}, 3 \mathrm{H}), 1.25(\mathrm{~s}, 9 \mathrm{H}) .{ }^{13} \mathbf{C}$ NMR (101 
$\left.\mathrm{MHz}, \mathrm{CDCl}_{3}\right) \delta 175.1,139.4,133.6,132.0,127.9,127.6,127.4,127.2,126.3,125.9,125.1,117.9$, 38.8, 38.6, 35.0, 27.5, 14.8. ESI-HR calcd for $\mathrm{C}_{20} \mathrm{H}_{26} \mathrm{NO}^{+}\left([\mathrm{M}+\mathrm{H}]^{+}\right)$296.2009, found 296.2000. IR $v\left(\mathrm{~cm}^{-1}\right) 3469,3342,3053,2961,1652,1493,1264,1197,733,703$. M.P. $107-108^{\circ} \mathrm{C}$.<smiles>C/C(=C\NC(=O)CC(C)(C)C)CCc1ccc(C(C)(C)C)cc1</smiles>

Enamide 1t: ${ }^{1} \mathbf{H}$ NMR $\left(400 \mathrm{MHz}, \mathrm{CDCl}_{3}\right) \delta 7.37-7.28(\mathrm{~m}, 2 \mathrm{H}), 7.12(\mathrm{~d}, J=8.3 \mathrm{~Hz}, 2 \mathrm{H}), 6.99(\mathrm{~d}$, $J=10.3 \mathrm{~Hz}, 1 \mathrm{H}), 6.65(\mathrm{dq}, J=10.3,1.3 \mathrm{~Hz}, 1 \mathrm{H}), 2.77-2.64(\mathrm{~m}, 2 \mathrm{H}), 2.36-2.29(\mathrm{~m}, 2 \mathrm{H}), 1.68(\mathrm{~d}$, $J=1.4 \mathrm{~Hz}, 3 \mathrm{H}), 1.31(\mathrm{~s}, 9 \mathrm{H}), 1.25(\mathrm{~s}, 9 \mathrm{H}) .{ }^{13} \mathbf{C}$ NMR $\left(101 \mathrm{MHz}, \mathrm{CDCl}_{3}\right) \delta 175.1,148.6,138.8$, $127.9,125.2,118.2,117.7,38.9,38.7,34.3,34.3,31.4,27.5,14.8$. ESI-HR calcd for $\mathrm{C}_{20} \mathrm{H}_{32} \mathrm{NO}^{+}$ $\left([\mathrm{M}+\mathrm{H}]^{+}\right)$302.2478, found 302.2470. IR $v\left(\mathrm{~cm}^{-1}\right)$ 3472, 3340, 2961, 2906, 1652, 1495, 1265, 1197, 734, 703. M.P. $116-117^{\circ} \mathrm{C}$.<smiles>CC(C)CCc1ccc(Cl)cc1</smiles>

Enamide 1u: ${ }^{1} \mathbf{H}$ NMR $\left(400 \mathrm{MHz}, \mathrm{CDCl}_{3}\right) \delta 7.12(\mathrm{ddt}, J=8.5,5.5,2.5 \mathrm{~Hz}, 2 \mathrm{H}), 7.04-6.89(\mathrm{~m}, 3 \mathrm{H})$, $6.61(\mathrm{dq}, J=10.3,1.4 \mathrm{~Hz}, 1 \mathrm{H}), 2.74-2.65(\mathrm{~m}, 2 \mathrm{H}), 2.34-2.25(\mathrm{~m}, 2 \mathrm{H}), 1.65(\mathrm{~d}, J=1.4 \mathrm{~Hz}, 3 \mathrm{H}), 1.24$ (s, 9H). ${ }^{13} \mathbf{C}$ NMR $\left(101 \mathrm{MHz}, \mathrm{CDCl}_{3}\right) \delta 175.2,162.5,160.0,137.4,129.6,129.6,118.0,117.5,115.2$, 115.0, 77.3, 77.0, 76.7, 38.9, 38.8, 33.9, 27.5, 14.8. ESI-HR calcd for $\mathrm{C}_{16} \mathrm{H}_{23} \mathrm{ClNO}^{+}\left([\mathrm{M}+\mathrm{H}]^{+}\right)$ 280.1463, found 280.1868. IR $v\left(\mathrm{~cm}^{-1}\right) 3470,3340,2961,2933,1650,1508,1492,1219,1196,825$, 734. M.P. $69-70^{\circ} \mathrm{C}$.<smiles>CC(=CNC(=O)CCc1ccc(OCc2ccccc2)cc1)CCc1ccccc1</smiles>

Enamide 1v: ${ }^{1} \mathbf{H}$ NMR $\left(400 \mathrm{MHz}, \mathrm{CDCl}_{3}\right) \delta 7.48-7.41(\mathrm{~m}, 2 \mathrm{H}), 7.38(\mathrm{ddd}, J=7.5,6.7,1.4 \mathrm{~Hz}$, 2H), $7.35-7.29(\mathrm{~m}, 1 \mathrm{H}), 7.14-7.05(\mathrm{~m}, 2 \mathrm{H}), 6.97(\mathrm{~d}, J=10.3 \mathrm{~Hz}, 1 \mathrm{H}), 6.93-6.86(\mathrm{~m}, 2 \mathrm{H}), 6.63$ (dq, $J=10.4,1.3 \mathrm{~Hz}, 1 \mathrm{H}), 5.04(\mathrm{~s}, 2 \mathrm{H}), 2.73-2.60(\mathrm{~m}, 2 \mathrm{H}), 2.29(\mathrm{dd}, J=9.5,6.7 \mathrm{~Hz}, 2 \mathrm{H}), 1.66(\mathrm{~d}$, $J=1.4 \mathrm{~Hz}, 3 \mathrm{H}), 1.25(\mathrm{~s}, 9 \mathrm{H}) .{ }^{13} \mathbf{C}$ NMR $\left(101 \mathrm{MHz}, \mathrm{CDCl}_{3}\right) \delta 175.1,157.0,137.2,134.2,129.2$, 128.5, 127.9, 127.4, 118.0, 117.8, 114.7, 70.0, 38.9, 38.8, 33.9, 27.5, 14.8. ESI-HR calcd for $\mathrm{C}_{23} \mathrm{H}_{30} \mathrm{NO}_{2}{ }^{+}\left([\mathrm{M}+\mathrm{H}]^{+}\right) 352.2271$, found 352.2262. IR $v\left(\mathrm{~cm}^{-1}\right) 3469,3350,2960,2931,1656,1509$, 1493, 1234, 1196, 734, 697. M.P. 114 - $115^{\circ} \mathrm{C}$. 
<smiles>COc1cc(CC/C(C)=C/NC(=O)C(C)(C)C)cc(OC)c1</smiles>

Enamide 1w: ${ }^{1} \mathrm{H}$ NMR $\left(400 \mathrm{MHz}, \mathrm{CDCl}_{3}\right) \delta 6.98(\mathrm{~d}, J=10.4 \mathrm{~Hz}, 1 \mathrm{H}), 6.64(\mathrm{dq}, J=10.3,1.3 \mathrm{~Hz}$, $1 \mathrm{H}), 6.34(\mathrm{~d}, J=2.3 \mathrm{~Hz}, 2 \mathrm{H}), 6.30(\mathrm{t}, J=2.3 \mathrm{~Hz}, 1 \mathrm{H}), 3.78(\mathrm{~s}, 6 \mathrm{H}), 2.72-2.60(\mathrm{~m}, 2 \mathrm{H}), 2.31(\mathrm{dd}$, $J=9.6,6.6 \mathrm{~Hz}, 2 \mathrm{H}), 1.66(\mathrm{~d}, J=1.4 \mathrm{~Hz}, 3 \mathrm{H}), 1.24(\mathrm{~s}, 9 \mathrm{H}) .{ }^{13} \mathbf{C} \mathbf{N M R}\left(101 \mathrm{MHz}, \mathrm{CDCl}_{3}\right) \delta 175.1$, $160.7,144.3,117.8,117.8,106.3,97.8,55.2,38.8,38.5,35.2,27.5,14.8$. ESI-HR calcd for $\mathrm{C}_{18} \mathrm{H}_{28} \mathrm{NO}_{3}{ }^{+}\left([\mathrm{M}+\mathrm{H}]^{+}\right)$306.2064, found 306.2055. IR $v\left(\mathrm{~cm}^{-1}\right)$ 3469, 2959, 1660, 1595, 1490, 1461 , 1204, 1149, 733, 700. M.P. $68-69^{\circ} \mathrm{C}$.<smiles>CC/C(=C\NC(=O)Br)CBr</smiles>

Enamide 1 $\mathbf{x}$ was prepared according to the general procedure starting from 4-phenyl-1-butyne $(1.30 \mathrm{~g}, 10.0 \mathrm{mmol})$, triethylaluminum $(1.0 \mathrm{M}$ solution in toluene, $20 \mathrm{mmol})$ and pivalamide $(2.02$ g, $20.0 \mathrm{mmol}$ ). The crude product was purified by flash chromatography on silica gel using PE/EtOAc as eluent: ${ }^{1} \mathrm{H}$ NMR $\left(400 \mathrm{MHz}, \mathrm{CDCl}_{3}\right) \delta 7.28(\mathrm{t}, J=7.5 \mathrm{~Hz}, 2 \mathrm{H}), 7.21-7.14(\mathrm{~m}, 3 \mathrm{H})$, $7.05(\mathrm{~s}, 1 \mathrm{H}), 6.62(\mathrm{~d}, J=10.4 \mathrm{~Hz}, 1 \mathrm{H}), 2.76-2.68(\mathrm{~m}, 2 \mathrm{H}), 2.37-2.28(\mathrm{~m}, 2 \mathrm{H}), 2.06(\mathrm{q}, J=7.6$ $\mathrm{Hz}, 2 \mathrm{H}), 1.24$ (s, 9H), 1.06 (t, $J=7.6 \mathrm{~Hz}, 3 \mathrm{H}) .{ }^{13} \mathbf{C}$ NMR $\left(101 \mathrm{MHz}, \mathrm{CDCl}_{3}\right) \delta 175.1,142.0,128.4$, 128.3, 125.9, 123.9, 117.2, 38.9, 36.2, 35.0, 27.5, 22.4, 12.2. ESI-HR calcd for $\mathrm{C}_{17} \mathrm{H}_{26} \mathrm{NO}^{+}\left([\mathrm{M}+\mathrm{H}]^{+}\right)$ 260.2009, found 260.2001. IR $v\left(\mathrm{~cm}^{-1}\right)$ 2962, 2928, 1660, 1494, 1265, 736, 700. M.P. $71-72{ }^{\circ} \mathrm{C}$.<smiles>C/C(=C/NC(=O)CBr)CBr</smiles>

Enamide 1a-Z was the same as the previous report ${ }^{6}$.

\section{Characterization of Products}

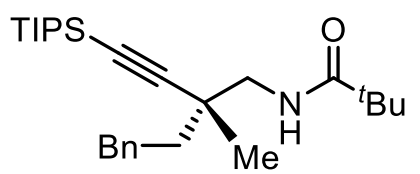

Amide 4a was prepared according to the general procedure starting from $1 \mathbf{a}(24.5 \mathrm{mg}, 0.10 \mathrm{mmol})$ and reaction mixture was heated at $80{ }^{\circ} \mathrm{C}$ for $24 \mathrm{~h}$. The crude product was purified by flash 
chromatography on silica gel $(\mathrm{PE} / \mathrm{EtOAc}=9: 1)$ and $\mathbf{4 a}$ was obtained as a colorless oil $(36.0 \mathrm{mg}$, yield $=84 \%) .{ }^{1} \mathbf{H}$ NMR $\left(400 \mathrm{MHz}, \mathrm{CDCl}_{3}\right) \delta 7.31-7.24(\mathrm{~m}, 2 \mathrm{H}), 7.22-7.14(\mathrm{~m}, 3 \mathrm{H}), 6.06(\mathrm{t}, J=$ $5.6 \mathrm{~Hz}, 1 \mathrm{H}), 3.38$ (dd, $J=12.9,6.5 \mathrm{~Hz}, 1 \mathrm{H}), 3.27$ (dd, $J=12.9,5.3 \mathrm{~Hz}, 1 \mathrm{H}), 2.79$ (dq, $J=13.1,7.4$, $6.5 \mathrm{~Hz}, 2 \mathrm{H}), 1.84-1.58(\mathrm{~m}, 2 \mathrm{H}), 1.24(\mathrm{~s}, 3 \mathrm{H}), 1.22(\mathrm{~s}, 9 \mathrm{H}), 1.11(\mathrm{~d}, J=3.8 \mathrm{~Hz}, 21 \mathrm{H}) .{ }^{13} \mathbf{C} \mathbf{N M R}$ $\left(101 \mathrm{MHz}, \mathrm{CDCl}_{3}\right) \delta 178.3,142.2,128.4,128.3,125.8,112.6,83.0,48.0,41.6,38.8,37.7,31.5$, 29.7, 27.6, 27.6, 24.4, 18.7, 11.2. ESI-HR calcd for $\mathrm{C}_{27} \mathrm{H}_{46} \mathrm{NOSi}^{+}\left([\mathrm{M}+\mathrm{H}]^{+}\right) 428.3343$, found 428.3333. IR $v\left(\mathrm{~cm}^{-1}\right) 3451,3379,2942,2864,2162,1662,1514,883,740,699,676,661 .[\alpha]^{25} \mathrm{D}=$ $+14.7\left(c=0.90, \mathrm{CHCl}_{3}\right)$ for a $92 \%$ ee sample. The enantiomeric purity of this compound was determined by HPLC analysis in comparison with authentic racemic material (Chiralpak ID, hexane : $\left.{ }^{i} \mathrm{PrOH}=99: 1,1.0 \mathrm{~mL} / \mathrm{min}, 210 \mathrm{~nm}\right): \mathrm{t}_{\text {major }}=17.009 \mathrm{~min}, \mathrm{t}_{\mathrm{minor}}=18.805 \mathrm{~min}$.<smiles>[Y10][C@@](C#CS[In])(CBr)CNC(=O)[AlH2]</smiles>

Amide $4 \mathbf{b}$ was prepared according to the general procedure starting from $1 \mathbf{b}(32.3 \mathrm{mg}, 0.10 \mathrm{mmol})$ and reaction mixture was heated at $80{ }^{\circ} \mathrm{C}$ for $24 \mathrm{~h}$. The crude product was purified by flash chromatography on silica gel $(\mathrm{PE} / \mathrm{EtOAc}=10: 1)$ and $\mathbf{4 b}$ was obtained as a white solid (32.1 $\mathrm{mg}$, yield = 64\%). ${ }^{1} \mathbf{H}$ NMR $\left(400 \mathrm{MHz}, \mathrm{CDCl}_{3}\right) \delta 7.32-7.25(\mathrm{~m}, 2 \mathrm{H}), 7.22-7.13(\mathrm{~m}, 3 \mathrm{H}), 6.05(\mathrm{~s}, 1 \mathrm{H})$, $3.37(\mathrm{dd}, J=12.9,6.6 \mathrm{~Hz}, 1 \mathrm{H}), 3.26(\mathrm{dd}, J=12.9,5.2 \mathrm{~Hz}, 1 \mathrm{H}), 2.79(\mathrm{dq}, J=12.7,7.3,6.3 \mathrm{~Hz}, 2 \mathrm{H})$, $2.04(\mathrm{~s}, 3 \mathrm{H}), 1.88(\mathrm{~d}, J=3.0 \mathrm{~Hz}, 6 \mathrm{H}), 1.81-1.64(\mathrm{~m}, 8 \mathrm{H}), 1.23(\mathrm{~s}, 3 \mathrm{H}), 1.11(\mathrm{~s}, 21 \mathrm{H}) .{ }^{13} \mathbf{C} \mathbf{~ N M R}$ $\left(101 \mathrm{MHz}, \mathrm{CDCl}_{3}\right) \delta 177.8,142.2,128.4,128.3,125.8,112.6,82.9,47.6,41.5,40.7,39.4,37.8$, 36.5, 31.5, 28.1, 24.4, 18.7, 11.2. ESI-HR calcd for $\mathrm{C}_{33} \mathrm{H}_{52} \mathrm{NOSi}^{+}\left([\mathrm{M}+\mathrm{H}]^{+}\right)$506.3813, found 506.3800. IR $v\left(\mathrm{~cm}^{-1}\right)$ 2910, 2864, 2360, 2341, 1656, 1514, 1455, 1264, 734, 701. M.P. 79 - $80{ }^{\circ} \mathrm{C}$. $[\alpha]^{25} \mathrm{D}=+9.43\left(c=1.21, \mathrm{CHCl}_{3}\right)$ for a $92 \%$ ee sample. The enantiomeric purity of this compound was determined by HPLC analysis in comparison with authentic racemic material (Chiralpak IC, hexane $\left.:{ }^{i} \mathrm{PrOH}=96: 4,1.0 \mathrm{~mL} / \mathrm{min}, 207 \mathrm{~nm}\right): \mathrm{t}_{\text {major }}=9.931 \mathrm{~min}, \mathrm{t}_{\text {minor }}=9.093 \mathrm{~min}$.<smiles>[Y19][C@@](C#CCSC)(CBr)CNC(=O)O</smiles>

Amide $4 \mathbf{c}$ was prepared according to the general procedure starting from $1 \mathbf{c}(27.1 \mathrm{mg}, 0.10 \mathrm{mmol})$ and reaction mixture was heated at $80{ }^{\circ} \mathrm{C}$ for $24 \mathrm{~h}$. The crude product was purified by flash 
chromatography on silica gel $(\mathrm{PE} / \mathrm{EtOAc}=10: 1)$ and $\mathbf{4 c}$ was obtained as a white solid (30.4 $\mathrm{mg}$, yield = 67\%). ${ }^{1} \mathbf{H}$ NMR $\left(400 \mathrm{MHz}, \mathrm{CDCl}_{3}\right) \delta 7.32-7.24(\mathrm{~m}, 2 \mathrm{H}), 7.17(\mathrm{~d}, J=7.9 \mathrm{~Hz}, 3 \mathrm{H}), 5.84(\mathrm{~s}$, $1 \mathrm{H}), 3.35(\mathrm{dd}, J=13.0,6.6 \mathrm{~Hz}, 1 \mathrm{H}), 3.26(\mathrm{dd}, J=12.9,5.4 \mathrm{~Hz}, 1 \mathrm{H}), 2.78(\mathrm{dq}, J=12.8,7.4,6.3 \mathrm{~Hz}$, 2H), $2.09(\mathrm{tt}, J=11.7,3.5 \mathrm{~Hz}, 1 \mathrm{H}), 1.89(\mathrm{~d}, J=12.9 \mathrm{~Hz}, 2 \mathrm{H}), 1.84-1.75(\mathrm{~m}, 3 \mathrm{H}), 1.71-1.62(\mathrm{~m}$, 2H), $1.43(\mathrm{qd}, J=12.0,3.2 \mathrm{~Hz}, 2 \mathrm{H}), 1.30-1.20(\mathrm{~m}, 6 \mathrm{H}), 1.10(\mathrm{~s}, 21 \mathrm{H}) .{ }^{13} \mathrm{C}$ NMR $(101 \mathrm{MHz}$, $\left.\mathrm{CDCl}_{3}\right) \delta 175.9,142.2,128.4,128.3,125.8,112.5,83.1,47.7,45.8,41.4,37.7,31.5,29.9,29.8,25.8$, 24.4, 18.7, 11.1. ESI-HR calcd for $\mathrm{C}_{29} \mathrm{H}_{48} \mathrm{NOSi}^{+}\left([\mathrm{M}+\mathrm{H}]^{+}\right) 454.3500$, found 454.3490. IR $v\left(\mathrm{~cm}^{-1}\right)$ 2933, 2864, 2361, 1668, 1515, 1264, 734, 701. M.P. $97-98^{\circ} \mathrm{C} .[\alpha]^{25} \mathrm{D}=+13.5\left(c=1.01, \mathrm{CHCl}_{3}\right)$ for an $88 \%$ ee sample. The enantiomeric purity of this compound was determined by HPLC analysis in comparison with authentic racemic material (Chiralpak IF, hexane : ${ }^{i} \mathrm{PrOH}=98: 2,1.0 \mathrm{~mL} / \mathrm{min}$, $208 \mathrm{~nm}$ ): $\mathrm{t}_{\text {major }}=17.003 \mathrm{~min}, \mathrm{t}_{\text {minor }}=19.202 \mathrm{~min}$.<smiles>CCCC(=O)NC[C@@](C)(CBr)CC#CS</smiles>

Amide 4d was prepared according to the general procedure starting from $1 \mathbf{d}(23.1 \mathrm{mg}, 0.10 \mathrm{mmol})$ and reaction mixture was heated at $80{ }^{\circ} \mathrm{C}$ for $24 \mathrm{~h}$. The crude product was purified by flash chromatography on silica gel $(\mathrm{PE} / \mathrm{EtOAc}=5: 1)$ and $\mathbf{4 d}$ was obtained as a pale yellow oil (22.8 $\mathrm{mg}$, yield $=55 \%) .{ }^{1} \mathbf{H}$ NMR $\left(400 \mathrm{MHz}, \mathrm{CDCl}_{3}\right) \delta 7.35-7.24(\mathrm{~m}, 2 \mathrm{H}), 7.18(\mathrm{dd}, J=7.8,6.0 \mathrm{~Hz}, 3 \mathrm{H})$, $5.82(\mathrm{t}, J=6.0 \mathrm{~Hz}, 1 \mathrm{H}), 3.37(\mathrm{dd}, J=13.0,6.6 \mathrm{~Hz}, 1 \mathrm{H}), 3.27(\mathrm{dd}, J=13.0,5.4 \mathrm{~Hz}, 1 \mathrm{H}), 2.79(\mathrm{dq}, J$ $=12.8,7.3,6.3 \mathrm{~Hz}, 2 \mathrm{H}), 2.37(\mathrm{p}, J=6.9 \mathrm{~Hz}, 1 \mathrm{H}), 1.77(\mathrm{ddd}, J=13.3,11.1,6.1 \mathrm{~Hz}, 1 \mathrm{H}), 1.72-1.61$ $(\mathrm{m}, 1 \mathrm{H}), 1.25(\mathrm{~s}, 3 \mathrm{H}), 1.18(\mathrm{dd}, J=6.9,2.9 \mathrm{~Hz}, 6 \mathrm{H}), 1.11(\mathrm{~d}, J=3.6 \mathrm{~Hz}, 21 \mathrm{H}) .{ }^{13} \mathbf{C} \mathbf{N M R}(101 \mathrm{MHz}$, $\left.\mathrm{CDCl}_{3}\right) \delta 176.8,142.2,128.4,128.4,125.8,112.5,83.1,47.9,41.5,37.7,35.9,31.5,24.4,19.7,19.6$, 18.7, 11.2. ESI-HR calcd for $\mathrm{C}_{26} \mathrm{H}_{44} \mathrm{NOSi}^{+}\left([\mathrm{M}+\mathrm{H}]^{+}\right) 414.3187$, found 414.3178. IR $v\left(\mathrm{~cm}^{-1}\right) 3309$, $2941,2965,1652,1462,883,699,677 .[\alpha]^{25} \mathrm{D}=+16.4\left(c=1.0, \mathrm{CHCl}_{3}\right)$ for a $92 \%$ ee sample. The enantiomeric purity of this compound was determined by HPLC analysis in comparison with authentic racemic material (Chiralpak ID, hexane : ${ }^{i} \mathrm{PrOH}=98.5: 1.5,1.0 \mathrm{~mL} / \mathrm{min}, 210 \mathrm{~nm}$ ): $\mathrm{t}_{\text {major }}=$ $17.969 \mathrm{~min}, \mathrm{t}_{\mathrm{minor}}=19.354 \mathrm{~min}$.<smiles>CC(=O)NC[C@](C)(C#CSC(C)C)CBr</smiles> 
Amide 4e was prepared according to the general procedure starting from 1e $(20.3 \mathrm{mg}, 0.10 \mathrm{mmol})$ and reaction mixture was heated at $80{ }^{\circ} \mathrm{C}$ for $24 \mathrm{~h}$. The crude product was purified by flash chromatography on silica gel $(\mathrm{PE} / \mathrm{EtOAc}=2: 1)$ and $\mathbf{4 e}$ was obtained as a colorless oil (6.6 mg, yield $=17 \%) .{ }^{1} \mathbf{H}$ NMR $\left(400 \mathrm{MHz}, \mathrm{CDCl}_{3}\right) \delta 7.27(\mathrm{~d}, \mathrm{~J}=8.3 \mathrm{~Hz}, 2 \mathrm{H}), 7.18(\mathrm{~d}, \mathrm{~J}=7.6 \mathrm{~Hz}, 3 \mathrm{H}), 5.77(\mathrm{t}, \mathrm{J}$ $=6.2 \mathrm{~Hz}, 1 \mathrm{H}), 3.31(\mathrm{qd}, \mathrm{J}=13.0,6.0 \mathrm{~Hz}, 2 \mathrm{H}), 2.79(\mathrm{dq}, \mathrm{J}=12.6,7.4,6.2 \mathrm{~Hz}, 2 \mathrm{H}), 2.00(\mathrm{~s}, 3 \mathrm{H})$, $1.79(\mathrm{ddd}, \mathrm{J}=17.2,9.3,4.6 \mathrm{~Hz}, 1 \mathrm{H}), 1.67$ (ddd, J = 13.3, 9.9, $6.4 \mathrm{~Hz}, 1 \mathrm{H}), 1.26(\mathrm{~s}, 3 \mathrm{H}), 1.10$ (d, $\mathrm{J}=$ $3.4 \mathrm{~Hz}, 21 \mathrm{H}) .{ }^{13} \mathbf{C}$ NMR $\left(101 \mathrm{MHz}, \mathrm{CDCl}_{3}\right) \delta 169.9,142.2,128.4,128.4,125.9,112.3,83.2,48.4$, 41.4, 37.5, 31.6, 24.5, 23.4, 18.7, 11.1. ESI-HR calcd for $\mathrm{C}_{24} \mathrm{H}_{40} \mathrm{NOSi}^{+}\left([\mathrm{M}+\mathrm{H}]^{+}\right) 386.2874$, found 386.2865. IR $v\left(\mathrm{~cm}^{-1}\right) 3294,2941,2864,2165,1654,1556,1461,883,675 .[\alpha]^{25}{ }_{\mathrm{D}}=+8.1(c=1.0$, $\mathrm{CHCl}_{3}$ ) for an $84 \%$ ee sample. The enantiomeric purity of this compound was determined by HPLC analysis in comparison with authentic racemic material (Chiralpak IC, hexane $:{ }^{i} \mathrm{PrOH}=98.5: 1.5$, $1.0 \mathrm{~mL} / \mathrm{min}, 210 \mathrm{~nm}): \mathrm{t}_{\text {major }}=73.713 \mathrm{~min}, \mathrm{t}_{\mathrm{minor}}=59.746 \mathrm{~min}$.<smiles>C[C@](C#CS[18F])(CBr)CNC(=O)c1ccccc1</smiles>

Amide 4f was prepared according to the general procedure starting from $\mathbf{1 f}(26.5 \mathrm{mg}, 0.10 \mathrm{mmol})$ and reaction mixture was heated at $80^{\circ} \mathrm{C}$ for $24 \mathrm{~h}$. The crude product was purified by flash chromatography on silica gel $(\mathrm{PE} / \mathrm{EtOAc}=2: 1)$ and $\mathbf{4 f}$ was obtained as a white solid $(4.0 \mathrm{mg}$, yield $=9 \%) .{ }^{1} \mathbf{H}$ NMR $\left(400 \mathrm{MHz}, \mathrm{CDCl}_{3}\right) \delta 7.82-7.74(\mathrm{~m}, 2 \mathrm{H}), 7.51(\mathrm{t}, J=7.3 \mathrm{~Hz}, 1 \mathrm{H}), 7.43(\mathrm{t}, J=7.5$ Hz, 2H), $7.30(\mathrm{~s}, 2 \mathrm{H}), 7.19(\mathrm{~d}, J=6.8 \mathrm{~Hz}, 3 \mathrm{H}), 6.59-6.42(\mathrm{~m}, 1 \mathrm{H}), 3.65-3.42(\mathrm{~m}, 2 \mathrm{H}), 2.86(\mathrm{tq}$, $J=12.8,7.6,6.7 \mathrm{~Hz}, 2 \mathrm{H}), 1.92-1.68(\mathrm{~m}, 2 \mathrm{H}), 1.33(\mathrm{~s}, 3 \mathrm{H}), 1.10(\mathrm{~s}, 21 \mathrm{H}) .{ }^{13} \mathrm{C}$ NMR $(101 \mathrm{MHz}$, $\left.\mathrm{CDCl}_{3}\right) \delta 167.4,142.1,134.6,131.5,128.6,128.5,128.4,126.8,125.9,112.4,48.6,41.6,37.9,31.6$, 24.6, 18.7, 11.2. ESI-HR calcd for $\mathrm{C}_{29} \mathrm{H}_{42} \mathrm{NOSi}^{+}\left([\mathrm{M}+\mathrm{H}]^{+}\right) 448.3030$, found 448.3019. IR $v\left(\mathrm{~cm}^{-1}\right)$ 3054, 1422, 1264, 896, 703. M.P. $80-81^{\circ} \mathrm{C} .[\alpha]^{25} \mathrm{D}=+22.3\left(c=1.0, \mathrm{CHCl}_{3}\right)$ for an $82 \%$ ee sample. The enantiomeric purity of this compound was determined by HPLC analysis in comparison with authentic racemic material (Chiralpak $\mathrm{IH}$, hexane : ${ }^{i} \mathrm{PrOH}=97: 3,1.0 \mathrm{~mL} / \mathrm{min}, 210 \mathrm{~nm}$ ): $\mathrm{t}_{\text {major }}=$ $9.418 \mathrm{~min}, \mathrm{t}_{\mathrm{minor}}=10.958 \mathrm{~min}$.<smiles>CCCC(=O)NC[C@@]([14CH3])(C#CS[18F])CCO</smiles> 
Amide $4 \mathbf{h}$ was prepared according to the general procedure starting from $1 \mathbf{h}(18.3 \mathrm{mg}, 0.10 \mathrm{mmol})$ and reaction mixture was heated at $80{ }^{\circ} \mathrm{C}$ for $48 \mathrm{~h}$. The crude product was purified by flash chromatography on silica gel $(\mathrm{PE} / \mathrm{EtOAc}=10: 1)$ and $\mathbf{4 h}$ was obtained as a light yellow oil (24.5 mg, yield = 67\%). ${ }^{1} \mathbf{H}$ NMR $\left(400 \mathrm{MHz}, \mathrm{CDCl}_{3}\right) \delta 5.99(\mathrm{~s}, 1 \mathrm{H}), 3.25(\mathrm{dd}, J=12.8,6.5 \mathrm{~Hz}, 1 \mathrm{H}), 3.14$ $(\mathrm{dd}, J=12.9,5.2 \mathrm{~Hz}, 1 \mathrm{H}), 1.50-1.35(\mathrm{~m}, 3 \mathrm{H}), 1.33-1.25(\mathrm{~m}, 1 \mathrm{H}), 1.17(\mathrm{~s}, 9 \mathrm{H}), 1.10(\mathrm{~s}, 3 \mathrm{H}), 1.02$ (s, 21H), $0.88(\mathrm{t}, J=6.9 \mathrm{~Hz}, 3 \mathrm{H}) .{ }^{13} \mathbf{C}$ NMR $\left(101 \mathrm{MHz}, \mathrm{CDCl}_{3}\right) \delta 178.3,113.2,82.2,48.2,41.7$, 38.8, 37.5, 27.6, 24.4, 18.6, 18.1, 14.6, 11.1. ESI-HR calcd for $\mathrm{C}_{22} \mathrm{H}_{44} \mathrm{NOSi}^{+}\left([\mathrm{M}+\mathrm{H}]^{+}\right) 366.3187$, found 366.3174. IR $v\left(\mathrm{~cm}^{-1}\right) 2959,2943,2866,1661,1514,1265,734 .[\alpha]^{25} \mathrm{D}=+3.95(c=0.760$, $\mathrm{CHCl}_{3}$ ) for a $91 \%$ ee sample. The enantiomeric purity of this compound was determined by HPLC analysis in comparison with authentic racemic material (Chiralpak IC, hexane : ${ }^{i} \mathrm{PrOH}=99: 1,0.5$ $\mathrm{mL} / \mathrm{min}, 220 \mathrm{~nm}$ ): $\mathrm{t}_{\text {major }}=22.744 \mathrm{~min}, \mathrm{t}_{\text {minor }}=22.035 \mathrm{~min}$.<smiles>CC(C)CC[C@](C)(C#CS[In])CNC(=O)CBr</smiles>

Amide 4i was prepared according to the general procedure starting from $1 \mathbf{i}(21.1 \mathrm{mg}, 0.10 \mathrm{mmol})$ and reaction mixture was heated at $80{ }^{\circ} \mathrm{C}$ for $48 \mathrm{~h}$. The crude product was purified by flash chromatography on silica gel $(\mathrm{PE} / \mathrm{EtOAc}=10: 1)$ and $\mathbf{4 i}$ was obtained as a pale yellow oil $(26.0 \mathrm{mg}$, yield $=66 \%) .{ }^{1} \mathbf{H}$ NMR $\left(400 \mathrm{MHz}, \mathrm{CDCl}_{3}\right) \delta 6.05(\mathrm{~s}, 1 \mathrm{H}), 3.30(\mathrm{dd}, J=12.9,6.5 \mathrm{~Hz}, 1 \mathrm{H}), 3.20(\mathrm{dd}$, $J=12.9,5.2 \mathrm{~Hz}, 1 \mathrm{H}), 1.56-1.43(\mathrm{~m}, 2 \mathrm{H}), 1.41-1.32(\mathrm{~m}, 3 \mathrm{H}), 1.22(\mathrm{~s}, 9 \mathrm{H}), 1.15(\mathrm{~s}, 3 \mathrm{H}), 1.07(\mathrm{~s}$, 21H), $0.88(\mathrm{~d}, J=6.6 \mathrm{~Hz}, 6 \mathrm{H}) .{ }^{13} \mathrm{C}$ NMR $\left(101 \mathrm{MHz}, \mathrm{CDCl}_{3}\right) \delta 178.3,113.1,82.3,48.3,38.8,37.6$, 37.1, 33.9, 28.4, 27.6, 24.4, 22.6, 22.5, 18.6, 11.2. ESI-HR calcd for $\mathrm{C}_{24} \mathrm{H}_{48} \mathrm{NOSi}^{+}\left([\mathrm{M}+\mathrm{H}]^{+}\right)$ 394.3500, found 394.3490. IR $v\left(\mathrm{~cm}^{-1}\right) 2957,2866,1663,1513,1463,1265,919,737,676 .[\alpha]^{25} \mathrm{D}$ $=+1.92\left(c=0.937, \mathrm{CHCl}_{3}\right)$ for a $90 \%$ ee sample. The enantiomeric purity of this compound was determined by HPLC analysis in comparison with authentic racemic material (Chiralpak IC, hexane : $\left.{ }^{i} \mathrm{PrOH}=98.5: 1.5,1.0 \mathrm{~mL} / \mathrm{min}, 210 \mathrm{~nm}\right): \mathrm{t}_{\text {major }}=31.829 \mathrm{~min}, \mathrm{t}_{\mathrm{minor}}=30.714 \mathrm{~min}$.

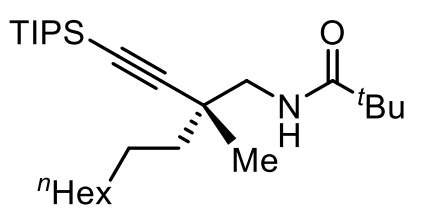

Amide $\mathbf{4} \mathbf{j}$ was prepared according to the general procedure starting from $\mathbf{1 j}$ ( $25.3 \mathrm{mg}, 0.10 \mathrm{mmol})$ and reaction mixture was heated at $80{ }^{\circ} \mathrm{C}$ for $48 \mathrm{~h}$. The crude product was purified by flash 
chromatography on silica gel $(\mathrm{PE} / \mathrm{EtOAc}=10: 1)$ and $\mathbf{4} \mathbf{j}$ was obtained as a pale yellow oil $(27.0 \mathrm{mg}$, yield $=62 \%) .{ }^{1} \mathbf{H}$ NMR $\left(400 \mathrm{MHz}, \mathrm{CDCl}_{3}\right) \delta 6.04(\mathrm{~s}, 1 \mathrm{H}), 3.30(\mathrm{dd}, J=12.9,6.5 \mathrm{~Hz}, 1 \mathrm{H}), 3.18(\mathrm{dd}$, $J=12.8,5.2 \mathrm{~Hz}, 1 \mathrm{H}), 1.50-1.40(\mathrm{~m}, 3 \mathrm{H}), 1.35-1.24(\mathrm{~m}, 11 \mathrm{H}), 1.21(\mathrm{~s}, 9 \mathrm{H}), 1.15(\mathrm{~s}, 3 \mathrm{H}), 1.07(\mathrm{~s}$, 21H), $0.88(\mathrm{t}, J=6.7 \mathrm{~Hz}, 3 \mathrm{H}) .{ }^{13} \mathbf{C} \mathbf{N M R}\left(101 \mathrm{MHz}, \mathrm{CDCl}_{3}\right) \delta 178.2,113.2,82.2,48.3,39.4,38.8$, 37.6, 31.9, 30.0, 29.5, 29.2, 27.6, 24.8, 24.4, 22.6, 18.6, 14.1, 11.2. ESI-HR calcd for $\mathrm{C}_{27} \mathrm{H}_{54} \mathrm{NOSi}^{+}$ $\left([\mathrm{M}+\mathrm{H}]^{+}\right)$436.3969, found 436.3954. IR $v\left(\mathrm{~cm}^{-1}\right)$ 3449, 2958, 2929, 2864, 1662, 1514, 1264, 883, 736. $[\alpha]^{25} \mathrm{D}=+3.25\left(c=0.893, \mathrm{CHCl}_{3}\right)$ for a $89 \%$ ee sample. The enantiomeric purity of this compound was determined by HPLC analysis in comparison with authentic racemic material (Chiralpak IC, hexane : ${ }^{i} \mathrm{PrOH}=99: 1,0.5 \mathrm{~mL} / \mathrm{min}, 210 \mathrm{~nm}$ ): $\mathrm{t}_{\text {major }}=25.131 \mathrm{~min}, \mathrm{t}_{\mathrm{minor}}=24.091 \mathrm{~min}$.<smiles>CCC[C@@](C)(C#CS[In])CC(=O)NCC(C)(C)CCCCl</smiles>

Amide 4k was prepared according to the general procedure starting from $1 \mathbf{k}(21.8 \mathrm{mg}, 0.10 \mathrm{mmol})$ and reaction mixture was heated at $80{ }^{\circ} \mathrm{C}$ for $48 \mathrm{~h}$. The crude product was purified by flash chromatography on silica gel $(\mathrm{PE} / \mathrm{EtOAc}=10: 1)$ and $\mathbf{4 k}$ was obtained as a light yellow oil (25.2 $\mathrm{mg}$, yield $=63 \%) .{ }^{1} \mathbf{H} \mathbf{~ N M R}\left(400 \mathrm{MHz}, \mathrm{CDCl}_{3}\right) \delta 5.98(\mathrm{~s}, 1 \mathrm{H}), 3.56-3.44(\mathrm{~m}, 2 \mathrm{H}), 3.22(\mathrm{qd}, J=$ 12.9, $6.0 \mathrm{~Hz}, 2 \mathrm{H}), 1.96-1.85(\mathrm{~m}, 2 \mathrm{H}), 1.64-1.52(\mathrm{~m}, 1 \mathrm{H}), 1.48-1.38(\mathrm{~m}, 1 \mathrm{H}), 1.16(\mathrm{~s}, 9 \mathrm{H}), 1.13$ (s, 3H), $1.02(\mathrm{~s}, 21 \mathrm{H}) .{ }^{13} \mathrm{C}$ NMR $\left(101 \mathrm{MHz}, \mathrm{CDCl}_{3}\right) \delta 178.3,112.2,83.0,48.1,45.2,38.8,37.3$, 36.3, 28.3, 27.6, 24.4, 18.6, 11.1. ESI-HR calcd for $\mathrm{C}_{22} \mathrm{H}_{43} \mathrm{NOSi}^{+}\left([\mathrm{M}+\mathrm{H}]^{+}\right) 400.2797$, found 400.2786. IR $v\left(\mathrm{~cm}^{-1}\right) 3451,2959,2944,2865,2360,2341,1663,1514,1462,1265,735 .[\alpha]^{25} \mathrm{D}=-$ $1.82\left(c=0.770, \mathrm{CHCl}_{3}\right)$ for a $90 \%$ ee sample. The enantiomeric purity of this compound was determined by HPLC analysis in comparison with authentic racemic material (Chiralpak IC, hexane : $\left.{ }^{i} \mathrm{PrOH}=99: 1,0.6 \mathrm{~mL} / \mathrm{min}, 203 \mathrm{~nm}\right): \mathrm{t}_{\text {major }}=48.244 \mathrm{~min}, \mathrm{t}_{\mathrm{minor}}=46.034 \mathrm{~min}$.

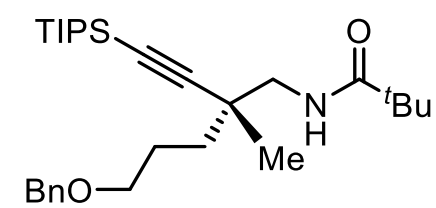

Amide $4 \mathbf{l}$ was prepared according to the general procedure starting from 11 (35.1 $\mathrm{mg}, 0.10 \mathrm{mmol})$ and reaction mixture was heated at $80{ }^{\circ} \mathrm{C}$ for $48 \mathrm{~h}$. The crude product was purified by flash chromatography on silica gel $(\mathrm{PE} / \mathrm{EtOAc}=4: 1)$ and $\mathbf{4 l}$ was obtained as a colorless oil (34.4 mg, yield = 73\%). ${ }^{1} \mathbf{H}$ NMR $\left(400 \mathrm{MHz}, \mathrm{CDCl}_{3}\right) \delta 7.33(\mathrm{~d}, J=5.7 \mathrm{~Hz}, 4 \mathrm{H}), 7.29-7.22(\mathrm{~m}, 1 \mathrm{H}), 6.03(\mathrm{t}$, 
$J=5.8 \mathrm{~Hz}, 1 \mathrm{H}), 4.49(\mathrm{~s}, 2 \mathrm{H}), 3.48(\mathrm{t}, J=6.4 \mathrm{~Hz}, 2 \mathrm{H}), 3.32(\mathrm{dd}, J=12.9,6.5 \mathrm{~Hz}, 1 \mathrm{H}), 3.21(\mathrm{dd}, J=$ 12.9, $5.3 \mathrm{~Hz}, 1 \mathrm{H}), 1.80$ (tt, $J=12.4,6.4 \mathrm{~Hz}, 2 \mathrm{H}), 1.57$ (ddd, $J=13.3,10.6,5.8 \mathrm{~Hz}, 1 \mathrm{H}), 1.44$ (ddd, $J=13.4,11.0,5.8 \mathrm{~Hz}, 1 \mathrm{H}), 1.24-1.18(\mathrm{~m}, 9 \mathrm{H}), 1.17(\mathrm{~s}, 3 \mathrm{H}), 1.07$ (d, $J=4.2 \mathrm{~Hz}, 21 \mathrm{H}) .{ }^{13} \mathbf{C}$ NMR $\left(101 \mathrm{MHz}, \mathrm{CDCl}_{3}\right) \delta 178.3,138.6,128.3,127.5,127.4,112.7,82.5,72.7,70.4,48.1,38.7,37.3$, 35.8, 27.5, 25.2, 24.3, 18.6, 11.1. ESI-HR calcd for $\mathrm{C}_{29} \mathrm{H}_{50} \mathrm{NO}_{2} \mathrm{Si}^{+}\left([\mathrm{M}+\mathrm{H}]^{+}\right) 472.3605$, found 472.3594. IR $v\left(\mathrm{~cm}^{-1}\right) 3018,2943,2865,1659,1515,1215,750,668 .[\alpha]^{25} \mathrm{D}=+16.9(c=0.35$, $\mathrm{CHCl}_{3}$ ) for a $92 \%$ ee sample. The enantiomeric purity of this compound was determined by HPLC analysis in comparison with authentic racemic material (Chiralpak ODH, hexane : ${ }^{i} \mathrm{PrOH}=99: 1$, $1.0 \mathrm{~mL} / \mathrm{min}, 210 \mathrm{~nm}): \mathrm{t}_{\text {major }}=12.096 \mathrm{~min}, \mathrm{t}_{\mathrm{minor}}=9.876 \mathrm{~min}$.

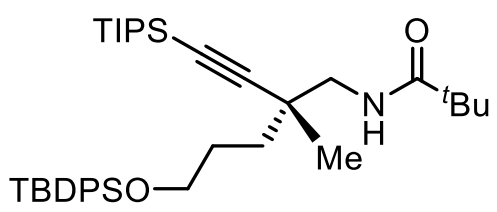

Amide 4m was prepared according to the general procedure starting from $1 \mathbf{m}(43.7 \mathrm{mg}, 0.10 \mathrm{mmol})$ and reaction mixture was heated at $80{ }^{\circ} \mathrm{C}$ for $48 \mathrm{~h}$. The crude product was purified by flash chromatography on silica gel $(\mathrm{PE} / \mathrm{EtOAc}=16: 1)$ and $\mathbf{4 m}$ was obtained as a colorless oil $(45.5 \mathrm{mg}$, yield $=73 \%) .{ }^{1} \mathbf{H}$ NMR $\left(400 \mathrm{MHz}, \mathrm{CDCl}_{3}\right) \delta 7.70-7.58(\mathrm{~m}, 4 \mathrm{H}), 7.48-7.31(\mathrm{~m}, 6 \mathrm{H}), 6.03(\mathrm{t}, J=$ $5.8 \mathrm{~Hz}, 1 \mathrm{H}), 3.67(\mathrm{td}, J=6.2,1.8 \mathrm{~Hz}, 2 \mathrm{H}), 3.33(\mathrm{dd}, J=12.8,6.6 \mathrm{~Hz}, 1 \mathrm{H}), 3.18(\mathrm{dd}, J=12.9,5.1$ $\mathrm{Hz}, 1 \mathrm{H}), 1.86-1.66(\mathrm{~m}, 2 \mathrm{H}), 1.66-1.55(\mathrm{~m}, 1 \mathrm{H}), 1.48-1.34(\mathrm{~m}, 1 \mathrm{H}), 1.21(\mathrm{~s}, 9 \mathrm{H}), 1.16(\mathrm{~s}, 3 \mathrm{H})$, $1.12-1.05(\mathrm{~m}, 21 \mathrm{H}), 1.04$ (s, 9H). ${ }^{13} \mathbf{C}$ NMR (101 MHz, $\left.\mathrm{CDCl}_{3}\right) \delta$ 178.3, 135.5, 134.0, 133.9, 129.5, $127.6,112.8,82.5,64.0,48.3,38.8,37.2,35.8,28.2,27.6,26.8,24.4,19.2,18.7,11.2$. ESI-HR calcd for $\mathrm{C}_{38} \mathrm{H}_{62} \mathrm{NO}_{2} \mathrm{Si}_{2}{ }^{+}\left([\mathrm{M}+\mathrm{H}]^{+}\right)$620.4314, found 620.4301. IR $v\left(\mathrm{~cm}^{-1}\right) 3018,2943,2864,1662$, $1515,1463,1215,1110,751,704,668 .[\alpha]^{25} \mathrm{D}=+2.7\left(c=1.0, \mathrm{CHCl}_{3}\right)$ for a $90 \%$ ee sample. The enantiomeric purity of this compound was determined by HPLC analysis in comparison with authentic racemic material (Chiralpak IC, hexane : ${ }^{i} \mathrm{PrOH}=99: 1,1.0 \mathrm{~mL} / \mathrm{min}, 210 \mathrm{~nm}$ ): $\mathrm{t}_{\text {major }}=$ $26.505 \mathrm{~min}, \mathrm{t}_{\mathrm{minor}}=25.521 \mathrm{~min}$.<smiles>CCCCC(=O)NC[C@@](C)(C#CS[In])CCCOC</smiles>

Amide $4 \mathbf{n}$ was prepared according to the general procedure starting from $1 \mathbf{n}(25.7 \mathrm{mg}, 0.10 \mathrm{mmol})$ and reaction mixture was heated at $80{ }^{\circ} \mathrm{C}$ for $48 \mathrm{~h}$. The crude product was purified by flash 
chromatography on silica gel $(\mathrm{PE} / \mathrm{EtOAc}=4: 1)$ and $\mathbf{4 n}$ was obtained as a colorless oil (24.6 mg, yield $=56 \%) .{ }^{1} \mathbf{H}$ NMR $\left(400 \mathrm{MHz}, \mathrm{CDCl}_{3}\right) \delta 6.02(\mathrm{t}, J=5.8 \mathrm{~Hz}, 1 \mathrm{H}), 4.64(\mathrm{~s}, 2 \mathrm{H}), 3.55(\mathrm{dq}, J=$ 12.5, $6.8 \mathrm{~Hz}, 4 \mathrm{H}), 3.31(\mathrm{dd}, J=12.9,6.5 \mathrm{~Hz}, 1 \mathrm{H}), 3.19(\mathrm{dd}, J=12.9,5.2 \mathrm{~Hz}, 1 \mathrm{H}), 1.74(\mathrm{dtd}, J=9.5$, 6.4, 3.3 Hz, 2H), $1.53(\mathrm{ddd}, J=13.2,9.9,6.4 \mathrm{~Hz}, 1 \mathrm{H}), 1.42(\mathrm{ddd}, J=13.2,10.5,6.3 \mathrm{~Hz}, 1 \mathrm{H}), 1.20$ (s, 12H), $1.16(\mathrm{~s}, 3 \mathrm{H}), 1.06(\mathrm{~d}, J=3.9 \mathrm{~Hz}, 21 \mathrm{H}) .{ }^{13} \mathrm{C} \mathbf{N M R}\left(101 \mathrm{MHz}, \mathrm{CDCl}_{3}\right) \delta 178.3,112.7,94.9$, 82.6, 67.8, 63.1, 48.1, 38.8, 37.3, 35.9, 27.5, 25.3, 24.3, 18.6, 15.1, 11.1. ESI-HR calcd for $\mathrm{C}_{25} \mathrm{H}_{50} \mathrm{NO}_{3} \mathrm{Si}^{+}\left([\mathrm{M}+\mathrm{H}]^{+}\right)$440.3554, found 440.3544. IR $v\left(\mathrm{~cm}^{-1}\right)$ 3016, 2944, 2866, 1662, 1517, $1215,748,668 .[\alpha]^{25} \mathrm{D}=+2.2\left(c=1.0, \mathrm{CHCl}_{3}\right)$ for a $90 \%$ ee sample. The enantiomeric purity of this compound was determined by HPLC analysis in comparison with authentic racemic material (Chiralpak IE, hexane : ${ }^{i} \operatorname{PrOH}=98: 2,1.0 \mathrm{~mL} / \mathrm{min}, 210 \mathrm{~nm}$ ): $\mathrm{t}_{\text {major }}=20.557 \mathrm{~min}, \mathrm{t}_{\text {minor }}=19.797 \mathrm{~min}$.<smiles>[Y19][C@](C#CS[InH])(CCCOC(C)=O)CNC(=O)CC(C)C</smiles>

Amide 40 was prepared according to the general procedure starting from $10(42.4 \mathrm{mg}, 0.10 \mathrm{mmol})$ and reaction mixture was heated at $80{ }^{\circ} \mathrm{C}$ for $24 \mathrm{~h}$. The crude product was purified by flash chromatography on silica gel $(\mathrm{PE} / \mathrm{EtOAc}=4: 1)$ and $\mathbf{4 o}$ was obtained as pale yellow oil $(25.4 \mathrm{mg}$, yield $=60 \%) .{ }^{1} \mathbf{H}$ NMR $\left(400 \mathrm{MHz}, \mathrm{CDCl}_{3}\right) \delta 6.01(\mathrm{t}, J=5.9 \mathrm{~Hz}, 1 \mathrm{H}), 4.05(\mathrm{t}, J=6.4 \mathrm{~Hz}, 2 \mathrm{H}), 3.29$ $(\mathrm{dd}, J=12.9,6.4 \mathrm{~Hz}, 1 \mathrm{H}), 3.21(\mathrm{dd}, J=12.9,5.5 \mathrm{~Hz}, 1 \mathrm{H}), 2.01(\mathrm{~s}, 3 \mathrm{H}), 1.80(\operatorname{tdd}, J=9.3,7.6,4.4$ $\mathrm{Hz}, 2 \mathrm{H}), 1.52$ (ddd, $J=13.2,10.0,6.5 \mathrm{~Hz}, 1 \mathrm{H}), 1.39$ (ddd, $J=13.4,10.5,6.5 \mathrm{~Hz}, 1 \mathrm{H}), 1.19$ (s, 9H), $1.15(\mathrm{~s}, 3 \mathrm{H}), 1.05(\mathrm{~d}, J=3.6 \mathrm{~Hz}, 21 \mathrm{H}) .{ }^{13} \mathrm{C}$ NMR $\left(101 \mathrm{MHz}, \mathrm{CDCl}_{3}\right) \delta 178.3,171.0,112.3,82.9$, 64.5, 48.1, 38.8, 37.3, 35.5, 27.5, 24.3, 24.2, 20.9, 18.6, 11.1. ESI-HR calcd for $\mathrm{C}_{24} \mathrm{H}_{46} \mathrm{NO}_{3} \mathrm{Si}^{+}$ $\left([\mathrm{M}+\mathrm{H}]^{+}\right)$424.3241, found 424.3231. IR $v\left(\mathrm{~cm}^{-1}\right)$ 3019, 2960, 2866, 1733, 1662, 1517, 1643, 1215, $754,668 .[\alpha]^{25} \mathrm{D}=-4.0\left(c=0.5, \mathrm{CHCl}_{3}\right)$ for a $90 \%$ ee sample. The enantiomeric purity of this compound was determined by HPLC analysis in comparison with authentic racemic material (Chiralpak ID, hexane : ${ }^{i} \mathrm{PrOH}=96: 4,1.0 \mathrm{~mL} / \mathrm{min}, 210 \mathrm{~nm}$ ): $\mathrm{t}_{\text {major }}=10.897 \mathrm{~min}, \mathrm{t}_{\mathrm{minor}}=10.030 \mathrm{~min}$.<smiles>CC(C)(C)CC(=O)NC[C@@](C)(C#CS[In])CCCOC(=O)OC(C)(C)C</smiles>

Amide $4 \mathbf{p}$ was prepared according to the general procedure starting from $1 \mathbf{p}(29.9 \mathrm{mg}, 0.10 \mathrm{mmol})$ 
and reaction mixture was heated at $80{ }^{\circ} \mathrm{C}$ for $24 \mathrm{~h}$. The crude product was purified by flash chromatography on silica gel $(\mathrm{PE} / \mathrm{EtOAc}=10: 1)$ and $\mathbf{4 p}$ was obtained as a colorless oil $(29.1 \mathrm{mg}$, yield $=60 \%) .{ }^{1} \mathbf{H}$ NMR $\left(400 \mathrm{MHz}, \mathrm{CDCl}_{3}\right) \delta 6.02(\mathrm{~s}, 1 \mathrm{H}), 4.14(\mathrm{t}, J=6.5 \mathrm{~Hz}, 2 \mathrm{H}), 3.91(\mathrm{~d}, J=6.7$ $\mathrm{Hz}, 2 \mathrm{H}), 3.32(\mathrm{dd}, J=12.9,6.5 \mathrm{~Hz}, 1 \mathrm{H}), 3.22(\mathrm{dd}, J=13.0,5.4 \mathrm{~Hz}, 1 \mathrm{H}), 2.03-1.93(\mathrm{~m}, 1 \mathrm{H}), 1.91$ $-1.81(\mathrm{~m}, 2 \mathrm{H}), 1.62-1.51(\mathrm{~m}, 1 \mathrm{H}), 1.50-1.39(\mathrm{~m}, 1 \mathrm{H}), 1.21(\mathrm{~s}, 9 \mathrm{H}), 1.17(\mathrm{~s}, 3 \mathrm{H}), 1.06(\mathrm{~s}, 21 \mathrm{H})$, $0.95(\mathrm{~d}, J=6.7 \mathrm{~Hz}, 6 \mathrm{H}) .{ }^{13} \mathbf{C} \mathbf{N M R}\left(101 \mathrm{MHz}, \mathrm{CDCl}_{3}\right) \delta 175.1,155.3,118.0,116.8,77.3,77.0,76.7$, 73.9, 67.2, 38.7, 32.6, 27.7, 27.4, 26.8, 18.8, 14.4. ESI-HR calcd for $\mathrm{C}_{27} \mathrm{H}_{52} \mathrm{NO}_{4} \mathrm{Si}^{+}\left([\mathrm{M}+\mathrm{H}]^{+}\right)$ 482.366, found 482.3645. IR $v\left(\mathrm{~cm}^{-1}\right) 3054,2961,2866,2361,2341,1742,1663,1515,1303,734$, 704. $[\alpha]^{25} \mathrm{D}=+4.49\left(c=1.18, \mathrm{CHCl}_{3}\right)$ for an $89 \%$ ee sample. The enantiomeric purity of this compound was determined by HPLC analysis in comparison with authentic racemic material (Chiralpak IC, hexane : $\mathrm{i}-\mathrm{PrOH}=97: 3,1.0 \mathrm{~mL} / \mathrm{min}, 207 \mathrm{~nm}$ ): $\mathrm{t}_{\text {major }}=26.457 \mathrm{~min}, \mathrm{t}_{\text {minor }}=23.232$ $\min$.

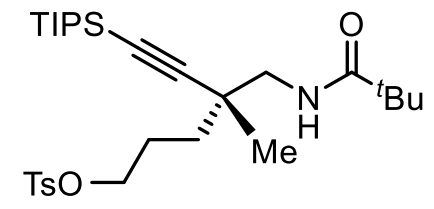

Amide $\mathbf{4 q}$ was prepared according to the general procedure starting from $1 \mathbf{q}(35.3 \mathrm{mg}, 0.10 \mathrm{mmol})$ and reaction mixture was heated at $80{ }^{\circ} \mathrm{C}$ for $24 \mathrm{~h}$. The crude product was purified by flash chromatography on silica gel $(\mathrm{PE} / \mathrm{EtOAc}=10: 1)$ and $\mathbf{4 q}$ was obtained as a pale yellow oil $(30.8 \mathrm{mg}$, yield $=57 \%) .{ }^{1} \mathbf{H}$ NMR $\left(400 \mathrm{MHz}, \mathrm{CDCl}_{3}\right) \delta 7.86-7.70(\mathrm{~m}, 2 \mathrm{H}), 7.33(\mathrm{~d}, J=8.1 \mathrm{~Hz}, 2 \mathrm{H}), 5.98(\mathrm{t}$, $J=6.0 \mathrm{~Hz}, 1 \mathrm{H}), 4.02(\mathrm{t}, J=6.3 \mathrm{~Hz}, 2 \mathrm{H}), 3.26(\mathrm{dd}, J=13.0,6.5 \mathrm{~Hz}, 1 \mathrm{H}), 3.16(\mathrm{dd}, J=13.0,5.6 \mathrm{~Hz}$, 1H), 2.44 (s, 3H), $1.91-1.76(\mathrm{~m}, 2 \mathrm{H}), 1.47$ (ddd, $J=13.3,10.5,5.9 \mathrm{~Hz}, 1 \mathrm{H}), 1.39-1.29(\mathrm{~m}, 1 \mathrm{H})$, $1.18(\mathrm{~s}, 9 \mathrm{H}), 1.11(\mathrm{~s}, 3 \mathrm{H}), 1.04(\mathrm{~d}, J=3.5 \mathrm{~Hz}, 21 \mathrm{H}) .{ }^{13} \mathbf{C} \mathbf{N M R}\left(101 \mathrm{MHz}, \mathrm{CDCl}_{3}\right) \delta 178.3,144.7$, $133.1,129.8,129.8,127.8,111.9,83.1,70.6,47.9,38.7,37.2,34.9,27.5,24.7,24.2,21.6,18.6,11.1$. ESI-HR calcd for $\mathrm{C}_{29} \mathrm{H}_{50} \mathrm{NO}_{4} \mathrm{SSi}^{+}\left([\mathrm{M}+\mathrm{H}]^{+}\right)$536.3224, found 536.3210. IR $v\left(\mathrm{~cm}^{-1}\right) 3019,2944$, $2866,1660,1517,1362,1214,750,668 .[\alpha]^{25} \mathrm{D}=+3.6\left(c=1.0, \mathrm{CHCl}_{3}\right)$ for an $86 \%$ ee sample. The enantiomeric purity of this compound was determined by HPLC analysis in comparison with authentic racemic material (Chiralpak IF, hexane : ${ }^{i} \mathrm{PrOH}=97: 3,1.0 \mathrm{~mL} / \mathrm{min}, 210 \mathrm{~nm}$ ): $\mathrm{t}_{\text {major }}=$ $45.915 \mathrm{~min}, \mathrm{t}_{\mathrm{minor}}=51.986 \mathrm{~min}$. 


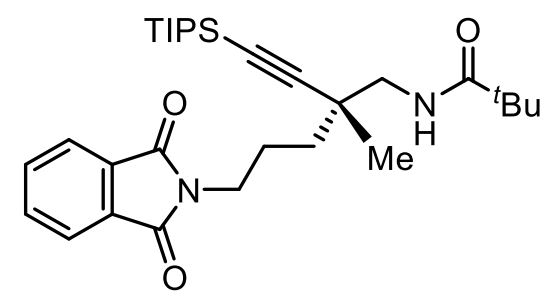

Amide $4 \mathbf{r}$ was prepared according to the general procedure starting from $1 \mathbf{r}(32.8 \mathrm{mg}, 0.10 \mathrm{mmol})$ and reaction mixture was heated at $80{ }^{\circ} \mathrm{C}$ for $48 \mathrm{~h}$. The crude product was purified by flash chromatography on silica gel $(\mathrm{PE} / \mathrm{EtOAc}=4: 1)$ and $\mathbf{4 r}$ was obtained as a pale yellow oil $(37.8 \mathrm{mg}$, yield $=74 \%) .{ }^{1} \mathbf{H}$ NMR $\left(400 \mathrm{MHz}, \mathrm{CDCl}_{3}\right) \delta 7.83(\mathrm{dd}, J=5.4,3.1 \mathrm{~Hz}, 2 \mathrm{H}), 7.71(\mathrm{dd}, J=5.4,3.1$ $\mathrm{Hz}, 2 \mathrm{H}), 5.98(\mathrm{t}, J=5.9 \mathrm{~Hz}, 1 \mathrm{H}), 3.70(\mathrm{t}, J=6.9 \mathrm{~Hz}, 2 \mathrm{H}), 3.30(\mathrm{dd}, J=12.9,6.7 \mathrm{~Hz}, 1 \mathrm{H}), 3.16(\mathrm{dd}$, $J=12.9,5.2 \mathrm{~Hz}, 1 \mathrm{H}), 1.88(\operatorname{tp}, J=10.0,6.9 \mathrm{~Hz}, 2 \mathrm{H}), 1.53(\mathrm{ddd}, J=13.3,10.4,6.3 \mathrm{~Hz}, 1 \mathrm{H}), 1.45-$ $1.35(\mathrm{~m}, 1 \mathrm{H}), 1.18(\mathrm{~s}, 9 \mathrm{H}), 1.13(\mathrm{~s}, 3 \mathrm{H}), 1.03(\mathrm{~d}, J=3.2 \mathrm{~Hz}, 21 \mathrm{H}) .{ }^{13} \mathbf{C} \mathbf{N M R}\left(101 \mathrm{MHz}, \mathrm{CDCl}_{3}\right) \delta$ $178.3,168.2,133.8,132.1,123.2,112.3,82.9,48.2,38.7,38.1,37.3,36.4,27.5,24.4,24.2,18.6$, 11.1. ESI-HR calcd for $\mathrm{C}_{30} \mathrm{H}_{47} \mathrm{~N}_{2} \mathrm{O}_{3} \mathrm{Si}^{+}\left([\mathrm{M}+\mathrm{H}]^{+}\right)$511.3350, found 511.3341. IR $v\left(\mathrm{~cm}^{-1}\right) 3449$, $3019,2943,2865,1713,1214,750,668 .[\alpha]^{25}=-2.5\left(c=1.0, \mathrm{CHCl}_{3}\right)$ for a $90 \%$ ee sample. The enantiomeric purity of this compound was determined by HPLC analysis in comparison with authentic racemic material (Chiralpak IF, hexane : ${ }^{i} \mathrm{PrOH}=95: 5,1.0 \mathrm{~mL} / \mathrm{min}, 210 \mathrm{~nm}$ ): $\mathrm{t}_{\text {major }}=$ $22.790 \mathrm{~min}, \mathrm{t}_{\mathrm{minor}}=24.902 \mathrm{~min}$.

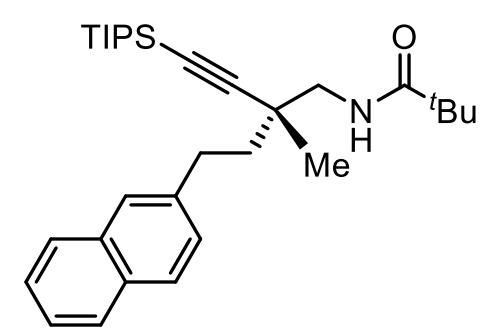

Amide 4s was prepared according to the general procedure starting from $1 \mathrm{~s}$ (29.5 $\mathrm{mg}, 0.10 \mathrm{mmol})$ and reaction mixture was heated at $80{ }^{\circ} \mathrm{C}$ for $24 \mathrm{~h}$. The crude product was purified by flash chromatography on silica gel $(\mathrm{PE} / \mathrm{EtOAc}=10: 1)$ and $\mathbf{4 s}$ was obtained as a colorless oil (37.3 mg, yield = 78\%). ${ }^{1} \mathbf{H}$ NMR $\left(400 \mathrm{MHz}, \mathrm{CDCl}_{3}\right) \delta 7.84-7.73(\mathrm{~m}, 3 \mathrm{H}), 7.62(\mathrm{~d}, J=1.6 \mathrm{~Hz}, 1 \mathrm{H}), 7.49-$ $7.38(\mathrm{~m}, 2 \mathrm{H}), 7.32(\mathrm{dd}, J=8.4,1.8 \mathrm{~Hz}, 1 \mathrm{H}), 6.18-5.96(\mathrm{~m}, 1 \mathrm{H}), 3.48-3.24(\mathrm{~m}, 2 \mathrm{H}), 2.97(\mathrm{tt}, J=$ 10.2, 7.1 Hz, 2H), $1.94-1.70(\mathrm{~m}, 2 \mathrm{H}), 1.28(\mathrm{~s}, 3 \mathrm{H}), 1.23(\mathrm{~s}, 9 \mathrm{H}), 1.18-1.03(\mathrm{~m}, 21 \mathrm{H}) .{ }^{13} \mathbf{C}$ NMR $\left(101 \mathrm{MHz}, \mathrm{CDCl}_{3}\right) \delta 178.3,139.7,133.6,132.0,128.0,127.6,127.4,127.2,126.3,125.9,125.1$, 112.6, 83.0, 48.0, 41.4, 38.8, 37.8, 31.7, 27.6, 24.4, 18.7, 11.2. ESI-HR calcd for $\mathrm{C}_{31} \mathrm{H}_{48} \mathrm{NOSi}^{+}$ $\left([\mathrm{M}+\mathrm{H}]^{+}\right)$478.3500, found 478.3488. IR $v\left(\mathrm{~cm}^{-1}\right) 3448,3018,2942,2865,1661,1513,1214,749$, 
668. $[\alpha]^{25} \mathrm{D}=+17.0\left(c=1.0, \mathrm{CHCl}_{3}\right)$ for a $91 \%$ ee sample. The enantiomeric purity of this compound was determined by HPLC analysis in comparison with authentic racemic material (Chiralpak ODH, hexane $\left.:{ }^{i} \mathrm{PrOH}=99: 1,1.0 \mathrm{~mL} / \mathrm{min}, 210 \mathrm{~nm}\right): \mathrm{t}_{\text {major }}=28.710 \mathrm{~min}, \mathrm{t}_{\text {minor }}=12.410 \mathrm{~min}$.

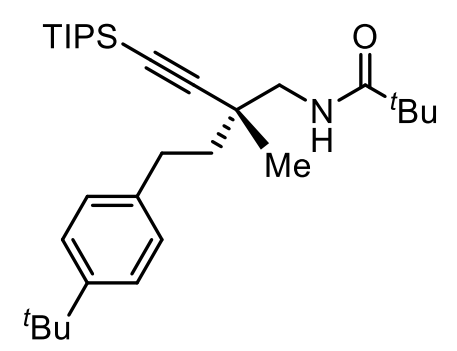

Amide 4t was prepared according to the general procedure starting from $1 \mathbf{t}(30.1 \mathrm{mg}, 0.10 \mathrm{mmol})$ and reaction mixture was heated at $80{ }^{\circ} \mathrm{C}$ for $24 \mathrm{~h}$. The crude product was purified by flash chromatography on silica gel $(\mathrm{PE} / \mathrm{EtOAc}=10: 1)$ and $\mathbf{4 t}$ was obtained as a colorless oil (37.7 $\mathrm{mg}$, yield $=78 \%) .{ }^{1} \mathbf{H}$ NMR $\left(400 \mathrm{MHz}, \mathrm{CDCl}_{3}\right) \delta 7.39-7.28(\mathrm{~m}, 2 \mathrm{H}), 7.18-7.09(\mathrm{~m}, 2 \mathrm{H}), 6.05(\mathrm{t}, J=$ $5.8 \mathrm{~Hz}, 1 \mathrm{H}), 3.38(\mathrm{dd}, J=12.9,6.7 \mathrm{~Hz}, 1 \mathrm{H}), 3.25(\mathrm{dd}, J=12.9,5.1 \mathrm{~Hz}, 1 \mathrm{H}), 2.87-2.68(\mathrm{~m}, 2 \mathrm{H})$, $1.84-1.59(\mathrm{~m}, 2 \mathrm{H}), 1.31(\mathrm{~s}, 10 \mathrm{H}), 1.24(\mathrm{~s}, 3 \mathrm{H}), 1.22(\mathrm{~s}, 9 \mathrm{H}), 1.11(\mathrm{~d}, J=3.9 \mathrm{~Hz}, 21 \mathrm{H}) .{ }^{13} \mathbf{C} \mathbf{N M R}$ $\left(101 \mathrm{MHz}, \mathrm{CDCl}_{3}\right) \delta 178.3,148.6,139.1,128.0,125.3,112.6,82.9,48.1,41.6,38.8,37.7,34.3$, 31.4, 31.3, 30.9, 27.6, 24.4, 18.7, 11.2. ESI-HR calcd for $\mathrm{C}_{31} \mathrm{H}_{54} \mathrm{NOSi}^{+}\left([\mathrm{M}+\mathrm{H}]^{+}\right) 484.3969$, found 484.3956. IR $v\left(\mathrm{~cm}^{-1}\right) 3019,2961,2866,1661,1516,1214,752,668 .[\alpha]^{25} \mathrm{D}=+7.8\left(c=0.26, \mathrm{CHCl}_{3}\right)$ for a $91 \%$ ee sample. The enantiomeric purity of this compound was determined by HPLC analysis in comparison with authentic racemic material (Chiralpak ODH, hexane $:{ }^{i} \mathrm{PrOH}=98: 2,1.0 \mathrm{~mL} / \mathrm{min}$, $210 \mathrm{~nm}): \mathrm{t}_{\mathrm{major}}=4.471 \mathrm{~min}, \mathrm{t}_{\mathrm{minor}}=4.067 \mathrm{~min}$.

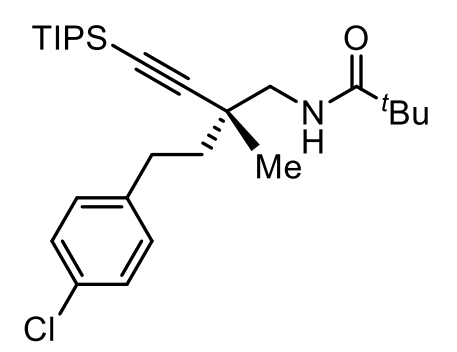

Amide $4 \mathbf{u}$ was prepared according to the general procedure starting from $1 \mathbf{u}(28.0 \mathrm{mg}, 0.10 \mathrm{mmol})$ and reaction mixture was heated at $80{ }^{\circ} \mathrm{C}$ for $48 \mathrm{~h}$. The crude product was purified by flash chromatography on silica gel $(\mathrm{PE} / \mathrm{EtOAc}=10: 1)$ and $\mathbf{4} \mathbf{u}$ was obtained as a white solid $(29.5 \mathrm{mg}$, yield $=64 \%) .{ }^{1} \mathbf{H}$ NMR $\left(400 \mathrm{MHz}, \mathrm{CDCl}_{3}\right) \delta 7.20-7.03(\mathrm{~m}, 2 \mathrm{H}), 7.03-6.87(\mathrm{~m}, 2 \mathrm{H}), 6.05(\mathrm{t}, J=$ $5.9 \mathrm{~Hz}, 1 \mathrm{H}), 3.36(\mathrm{dd}, J=13.0,6.5 \mathrm{~Hz}, 1 \mathrm{H}), 3.27(\mathrm{dd}, J=12.9,5.4 \mathrm{~Hz}, 1 \mathrm{H}), 2.76(\mathrm{tt}, J=11.1,7.3$ 
$\mathrm{Hz}, 2 \mathrm{H}), 1.80-1.51(\mathrm{~m}, 2 \mathrm{H}), 1.23(\mathrm{~s}, 3 \mathrm{H}), 1.21(\mathrm{~s}, 9 \mathrm{H}), 1.10(\mathrm{~d}, J=3.5 \mathrm{~Hz}, 21 \mathrm{H}) .{ }^{13} \mathbf{C}$ NMR $(101$ $\left.\mathrm{MHz}, \mathrm{CDCl}_{3}\right) \delta 178.3,162.5,160.0,137.8,137.8,129.7,129.6,115.2,115.0,112.5,83.0,47.9,41.6$, 38.8, 37.7, 30.7, 27.6, 24.4, 18.7, 11.2. ESI-HR calcd for $\mathrm{C}_{27} \mathrm{H}_{45} \mathrm{ClNOSi}^{+}\left([\mathrm{M}+\mathrm{H}]^{+}\right) 462.2953$, found 462.3361. IR $v\left(\mathrm{~cm}^{-1}\right) 3018,2944,2866,1661,1510,1215,751,668$. M.P. $59-60{ }^{\circ} \mathrm{C} .[\alpha]^{25} \mathrm{D}$ $=+8.9\left(c=0.70, \mathrm{CHCl}_{3}\right)$ for a $90 \%$ ee sample. The enantiomeric purity of this compound was determined by HPLC analysis in comparison with authentic racemic material (Chiralpak IC, hexane : $\left.{ }^{i} \mathrm{PrOH}=98: 2,1.0 \mathrm{~mL} / \mathrm{min}, 210 \mathrm{~nm}\right): \mathrm{t}_{\text {major }}=12.901 \mathrm{~min}, \mathrm{t}_{\mathrm{minor}}=11.894 \mathrm{~min}$.

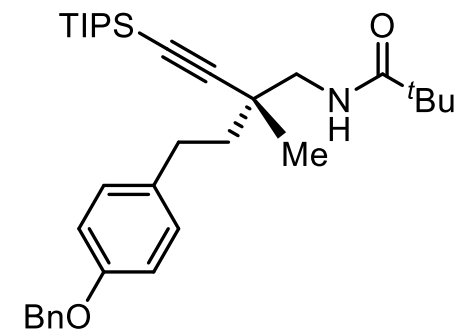

Amide $4 \mathbf{v}$ was prepared according to the general procedure starting from $1 \mathbf{v}(35.1 \mathrm{mg}, 0.10 \mathrm{mmol})$ and reaction mixture was heated at $80{ }^{\circ} \mathrm{C}$ for $24 \mathrm{~h}$. The crude product was purified by flash chromatography on silica gel $(\mathrm{PE} / \mathrm{EtOAc}=5: 1)$ and $\mathbf{4 v}$ was obtained as a light yellow oil $(41.2 \mathrm{mg}$, yield $=77 \%) .{ }^{1} \mathbf{H}$ NMR $\left(400 \mathrm{MHz}, \mathrm{CDCl}_{3}\right) \delta 7.47-7.35(\mathrm{~m}, 4 \mathrm{H}), 7.35-7.29(\mathrm{~m}, 1 \mathrm{H}), 7.14-7.05$ (m, 2H), $6.97-6.81(\mathrm{~m}, 2 \mathrm{H}), 6.05(\mathrm{t}, J=5.9 \mathrm{~Hz}, 1 \mathrm{H}), 5.05(\mathrm{~s}, 2 \mathrm{H}), 3.37$ (dd, $J=12.9,6.6 \mathrm{~Hz}, 1 \mathrm{H})$, $3.26(\mathrm{dd}, J=12.9,5.2 \mathrm{~Hz}, 1 \mathrm{H}), 2.74(\mathrm{dq}, J=12.9,7.5,6.4 \mathrm{~Hz}, 2 \mathrm{H}), 1.82-1.54(\mathrm{~m}, 2 \mathrm{H}), 1.23(\mathrm{~s}$, 3H), $1.22(\mathrm{~s}, 9 \mathrm{H}), 1.11(\mathrm{~d}, J=3.7 \mathrm{~Hz}, 21 \mathrm{H}) .{ }^{13} \mathbf{C} \mathbf{~ N M R}\left(101 \mathrm{MHz}, \mathrm{CDCl}_{3}\right) \delta$ 178.3, 157.0, 137.2, 134.6, 129.2, 128.5, 127.8, 127.4, 114.8, 112.6, 82.9, 70.0, 48.0, 41.8, 38.8, 37.7, 30.6, 27.6, 24.4, 18.7, 11.2. ESI-HR calcd for $\mathrm{C}_{34} \mathrm{H}_{52} \mathrm{NO}_{2} \mathrm{Si}^{+}\left([\mathrm{M}+\mathrm{H}]^{+}\right)$534.3762, found 534.3750. IR $v\left(\mathrm{~cm}^{-1}\right) 3018$, $2928,2865,1661,1511,1215,745,668 .[\alpha]^{25} \mathrm{D}=+9.0\left(c=1.0, \mathrm{CHCl}_{3}\right)$ for an $89 \%$ ee sample. The enantiomeric purity of this compound was determined by HPLC analysis in comparison with authentic racemic material (Chiralpak ODH, hexane $:{ }^{i} \mathrm{PrOH}=99: 1,1.0 \mathrm{~mL} / \mathrm{min}, 210 \mathrm{~nm}$ ): $\mathrm{t}_{\text {major }}=$ $27.856 \mathrm{~min}, \mathrm{t}_{\mathrm{minor}}=30.792 \mathrm{~min}$.<smiles>[M][C@](C#CS[InH])(CCc1cc(OC)cc(OC)c1)CNC(=O)CC(C)(C)C</smiles> 
Amide 4w was prepared according to the general procedure starting from $\mathbf{1 w}(30.5 \mathrm{mg}, 0.10 \mathrm{mmol})$ and reaction mixture was heated at $80{ }^{\circ} \mathrm{C}$ for $24 \mathrm{~h}$. The crude product was purified by flash chromatography on silica gel $(\mathrm{PE} / \mathrm{EtOAc}=5: 1)$ and $\mathbf{4 w}$ was obtained as a colorless oil $(30.7 \mathrm{mg}$, yield $=63 \%) .{ }^{1} \mathbf{H}$ NMR $\left(400 \mathrm{MHz}, \mathrm{CDCl}_{3}\right) \delta 6.34(\mathrm{~d}, J=2.3 \mathrm{~Hz}, 2 \mathrm{H}), 6.30(\mathrm{t}, J=2.3 \mathrm{~Hz}, 1 \mathrm{H}), 6.04$ (t, $J=5.9 \mathrm{~Hz}, 1 \mathrm{H}), 3.77(\mathrm{~s}, 6 \mathrm{H}), 3.37(\mathrm{dd}, J=13.0,6.6 \mathrm{~Hz}, 1 \mathrm{H}), 3.25(\mathrm{dd}, J=12.9,5.2 \mathrm{~Hz}, 1 \mathrm{H})$, $2.73(\mathrm{dq}, J=12.8,7.2,6.3 \mathrm{~Hz}, 2 \mathrm{H}), 1.84-1.57(\mathrm{~m}, 2 \mathrm{H}), 1.23(\mathrm{~s}, 3 \mathrm{H}), 1.21(\mathrm{~s}, 9 \mathrm{H}), 1.10(\mathrm{~d}, J=3.7$ $\mathrm{Hz}, 21 \mathrm{H}) .{ }^{13} \mathrm{C}$ NMR $\left(101 \mathrm{MHz}, \mathrm{CDCl}_{3}\right) \delta 178.3,160.8,144.7,112.6,106.4,98.0,82.9,55.2,48.0$, 41.4, 38.8, 37.7, 31.9, 27.6, 24.4, 18.7, 11.2. ESI-HR calcd for $\mathrm{C}_{29} \mathrm{H}_{50} \mathrm{NO}_{3} \mathrm{Si}^{+}\left([\mathrm{M}+\mathrm{H}]^{+}\right) 488.3554$, found 488.3544. IR $v\left(\mathrm{~cm}^{-1}\right) 3019,2400,1657,1597,1518,1463,1214,745,668 .[\alpha]^{25} \mathrm{D}=+10.6(c$ $=1.0, \mathrm{CHCl}_{3}$ ) for a $92 \%$ ee sample. The enantiomeric purity of this compound was determined by HPLC analysis in comparison with authentic racemic material (Chiralpak IC, hexane : ${ }^{i} \mathrm{PrOH}=97: 3$, $1.0 \mathrm{~mL} / \mathrm{min}, 210 \mathrm{~nm}): \mathrm{t}_{\text {major }}=28.694 \mathrm{~min}, \mathrm{t}_{\text {minor }}=25.703 \mathrm{~min}$.<smiles>CC[C@](C#CCS)(CBr)CNC(=O)CBr</smiles>

Amide 4x was prepared according to the general procedure starting from $\mathbf{1 x}(13.0 \mathrm{mg}, 0.050 \mathrm{mmol})$ and alkyne (45 uL, $0.20 \mathrm{mmol}$ ) using ligand $\mathbf{L 1 0}$ (442905-33-1) and reaction mixture was heated at $80^{\circ} \mathrm{C}$ for $48 \mathrm{~h}$. The crude product was purified by flash chromatography on silica gel $(\mathrm{PE} / \mathrm{EtOAc}=$ $10: 1)$ and $\mathbf{4 x}$ was obtained as a colourless oil $(11.1 \mathrm{mg}$, yield $=50 \%) .{ }^{\mathbf{1}} \mathbf{H} \mathbf{~ N M R}\left(400 \mathrm{MHz}, \mathrm{CDCl}_{3}\right) \delta$ $7.29(\mathrm{~d}, \mathrm{~J}=7.7 \mathrm{~Hz}, 2 \mathrm{H}), 7.18(\mathrm{td}, \mathrm{J}=7.3,6.7,1.5 \mathrm{~Hz}, 3 \mathrm{H}), 6.00(\mathrm{~s}, 1 \mathrm{H}), 3.47-3.21(\mathrm{~m}, 2 \mathrm{H}), 2.85$ $-2.58(\mathrm{~m}, 2 \mathrm{H}), 1.80-1.66(\mathrm{~m}, 2 \mathrm{H}), 1.62-1.51(\mathrm{~m}, 2 \mathrm{H}), 1.21(\mathrm{~s}, 9 \mathrm{H}), 1.10(\mathrm{~d}, \mathrm{~J}=3.6 \mathrm{~Hz}, 21 \mathrm{H})$, $1.06-0.96$ (m, 3H). ${ }^{13} \mathbf{C}$ NMR (101 MHz, $\left.\mathrm{CDCl}_{3}\right) \delta 178.3,142.4,128.5,128.4,125.9,111.8,84.0$, 45.3, 41.6, 38.8, 38.5, 31.2, 29.4, 27.6, 18.7, 18.7, 11.2, 8.8. ESI-HR calcd for $\mathrm{C}_{28} \mathrm{H}_{48} \mathrm{NOSi}^{+}$ $\left([\mathrm{M}+\mathrm{H}]^{+}\right)$442.3500, found 442.3488. IR $v\left(\mathrm{~cm}^{-1}\right)$ 3054, 2927, 2361, 2341, 1264, 896, 734, 704. $[\alpha]^{25}=-11.5\left(c=0.555, \mathrm{CHCl}_{3}\right)$ for a $98 \%$ ee sample. The enantiomeric purity of this compound was determined by HPLC analysis in comparison with authentic racemic material (Chiralpak ID, hexane $\left.:{ }^{i} \mathrm{PrOH}=98: 2,0.7 \mathrm{~mL} / \mathrm{min}, 205 \mathrm{~nm}\right): \mathrm{t}_{\text {major }}=10.985 \mathrm{~min}, \mathrm{t}_{\text {minor }}=12.097 \mathrm{~min}$.

\section{Diversification of Products}


<smiles>C#C[C@](C)(CBr)CNC(=O)Br</smiles>

Amide 5a: To a solution of $4 \mathbf{a}(0.1 \mathrm{mmol}, 1.0$ equiv) in THF $(0.40 \mathrm{~mL})$ was added TBAF $(0.20$ mmol, 2.0 equiv). The solution was stirred at rt. After the reaction was completed, the solution was extracted with DCM and washed with $1 \mathrm{M} \mathrm{HCl}$ solution. The organic extract was dried over $\mathrm{Na}_{2} \mathrm{SO}_{4}$ and concentrated in vacuo. The crude product was purified by flash chromatography on silica gel with $\mathrm{PE} /$ EtOAc mixture as eluent and $\mathbf{5 a}$ was obtained as a colorless solid (26.9 $\mathrm{mg}, 99 \%$ yield). ${ }^{1} \mathbf{H}$ NMR $\left(400 \mathrm{MHz}, \mathrm{CDCl}_{3}\right) \delta 7.32-7.24(\mathrm{~m}, 2 \mathrm{H}), 7.19(\mathrm{~d}, \mathrm{~J}=7.2 \mathrm{~Hz}, 3 \mathrm{H}), 6.01(\mathrm{~s}, 1 \mathrm{H}), 3.33(\mathrm{qd}, \mathrm{J}$ $=13.1,6.1 \mathrm{~Hz}, 2 \mathrm{H}), 2.86-2.66(\mathrm{~m}, 2 \mathrm{H}), 2.27(\mathrm{~s}, 1 \mathrm{H}), 1.83-1.73(\mathrm{~m}, 1 \mathrm{H}), 1.73-1.63(\mathrm{~m}, 1 \mathrm{H})$, $1.23(\mathrm{~d}, \mathrm{~J}=4.9 \mathrm{~Hz}, 12 \mathrm{H}) .{ }^{13} \mathrm{C}$ NMR $\left(101 \mathrm{MHz}, \mathrm{CDCl}_{3}\right) \delta 178.4,142.0,128.4,128.4,125.9,88.1$, 71.0, 47.6, 41.1, 38.9, 36.8, 31.2, 27.6, 24.2. ESI-HR calcd for $\mathrm{C}_{18} \mathrm{H}_{26} \mathrm{NO}^{+}\left([\mathrm{M}+\mathrm{H}]^{+}\right) 272.2009$, found 272.2002. IR $v\left(\mathrm{~cm}^{-1}\right) 3305,3018,2967,1658,1519,1215,749,668 .[\alpha]^{25} \mathrm{D}=+2.0(c=1.0$, $\mathrm{CHCl}_{3}$ ) for a $92 \%$ ee sample. The enantiomeric purity of this compound was determined by HPLC analysis in comparison with authentic racemic material (Chiralpak IC, hexane : ${ }^{i} \mathrm{PrOH}=99: 1,1.0$ $\mathrm{mL} / \mathrm{min}, 210 \mathrm{~nm}$ ): $\mathrm{t}_{\text {major }}=90.357 \mathrm{~min}, \mathrm{t}_{\text {minor }}=84.506 \mathrm{~min}$.
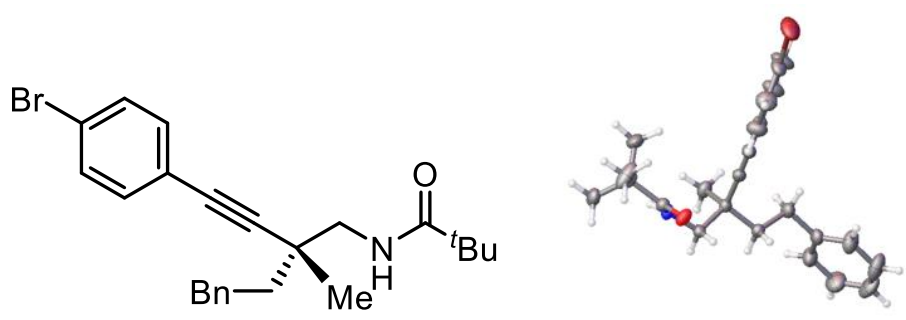

Amide 5b: In an argon filled glovebox, 5a (0.10 mmol, 1.0 equiv), $\mathrm{PdCl}_{2}\left(\mathrm{PPh}_{3}\right)_{2}(0.045 \mathrm{mmol}, 0.45$ equiv), $\mathrm{CuI}$ (0.045 mmol, 0.45 equiv) were weighed into a one-dram screw-capped vial. Subsequently, THF (1.0 mL), NEt $3(1.0 \mathrm{~mL})$ and 1-bromo-4-iodobenzene ( $0.12 \mathrm{mmol}, 1.2$ equiv) were added. The vial was capped with a Teflon-lined screw cap and the resulting solution was then removed from the glovebox, placed in a pre-heated aluminum block at $60{ }^{\circ} \mathrm{C}$ for $12 \mathrm{~h}$. The reaction mixture was concentrated and purified directly by column chromatography on silica gel with $\mathrm{PE} /$ EtOAc mixture as eluent and $\mathbf{5 b}$ was obtained as a white solid (35.2 $\mathrm{mg}, 83 \%$ yield). Single crystal suitable for X-ray analysis was obtained from slow diffusion of petroleum ether into a solution of $\mathbf{5 b}$ in DCM at room temperature. Data for the crystal structures reported in this paper is deposited at the Cambridge Crystallographic Data Centre (CCDC) under the deposition numbers 
CCDC 1971888. ${ }^{1} \mathbf{H}$ NMR (400 MHz, $\left.\mathrm{CDCl}_{3}\right) \delta 7.48-7.42(\mathrm{~m}, 2 \mathrm{H}), 7.31-7.23(\mathrm{~m}, 4 \mathrm{H}), 7.23-$ $7.15(\mathrm{~m}, 3 \mathrm{H}), 6.01(\mathrm{~s}, 1 \mathrm{H}), 3.48-3.33(\mathrm{~m}, 2 \mathrm{H}), 2.89-2.76(\mathrm{~m}, 2 \mathrm{H}), 1.92-1.69(\mathrm{~m}, 2 \mathrm{H}), 1.31(\mathrm{~s}$, 3H), 1.23 (s, 9H). ${ }^{13}$ C NMR (101 MHz, $\left.\mathrm{CDCl}_{3}\right) \delta$ 178.3, 142.0, 133.0, 131.6, 128.4, 128.3, 125.9, 122.2, 122.0, 94.4, 82.4, 47.7, 41.2, 38.8, 37.5, 31.4, 27.6, 24.3. ESI-HR calcd for $\mathrm{C}_{24} \mathrm{H}_{28} \mathrm{BrNNaOSi}^{+}\left([\mathrm{M}+\mathrm{Na}]^{+}\right)$448.1246, found 448.1242. IR $v\left(\mathrm{~cm}^{-1}\right) 3454,3374,2964,1659,1515$, $1485,1264,733,701$. M.P. $73-74^{\circ} \mathrm{C} .[\alpha]^{25} \mathrm{D}=+53.2\left(c=1.76, \mathrm{CHCl}_{3}\right)$ for a $92 \%$ ee sample. The enantiomeric purity of this compound was determined by HPLC analysis in comparison with authentic racemic material (Chiralpak IA, hexane $:{ }^{i} \operatorname{PrOH}=99: 1,1.0 \mathrm{~mL} / \mathrm{min}, 245 \mathrm{~nm}$ ): $\mathrm{t}_{\text {major }}=$ $31.400 \mathrm{~min}, \mathrm{t}_{\mathrm{minor}}=40.195 \mathrm{~min}$.<smiles>C=CC(CBr)(CBr)CNC(=O)Br</smiles>

Amide 5c: 5a ( $0.10 \mathrm{mmol}, 1.0$ equiv) and Lindlar's catalyst (palladium, $5 \%$ on calcium carbonate, lead poisoned, $2.7 \mathrm{mg}$ ) were placed in a $10 \mathrm{~mL}$ Schlenk tube. The tube was evacuated and refilled with hydrogen through a hydrogen balloon. After addition of $1.0 \mathrm{~mL}$ of EtOAc, the mixture was stirred at room temperature for $24 \mathrm{~h}$ under balloon pressure of hydrogen. The reaction mixture was filtered through Celite and concentrated. The residue was then purified directly by column chromatography on silica gel with PE/EtOAc mixture as eluent and $\mathbf{5} \mathbf{c}$ was obtained as a colorless oil (25.2 mg, 92\% yield). ${ }^{1} \mathbf{H}$ NMR $\left(400 \mathrm{MHz}, \mathrm{CDCl}_{3}\right) \delta 7.33-7.23$ (m, 2H), 7.17 (td, $J=7.4,6.8$, $1.6 \mathrm{~Hz}, 3 \mathrm{H}), 5.77$ (dd, $J=17.6,10.8 \mathrm{~Hz}, 1 \mathrm{H}), 5.61(\mathrm{~s}, 1 \mathrm{H}), 5.21$ (dd, $J=10.8,1.2 \mathrm{~Hz}, 1 \mathrm{H}), 5.07$ (dd, $J=17.6,1.2 \mathrm{~Hz}, 1 \mathrm{H}), 3.33(\mathrm{dd}, J=13.3,6.8 \mathrm{~Hz}, 1 \mathrm{H}), 3.12(\mathrm{dd}, J=13.3,4.9 \mathrm{~Hz}, 1 \mathrm{H}), 2.66-2.46$ $(\mathrm{m}, 2 \mathrm{H}), 1.71-1.55(\mathrm{~m}, 2 \mathrm{H}), 1.18(\mathrm{~s}, 9 \mathrm{H}), 1.06(\mathrm{~s}, 3 \mathrm{H}) .{ }^{13} \mathbf{C} \mathbf{N M R}\left(101 \mathrm{MHz}, \mathrm{CDCl}_{3}\right) \delta 178.2$, 144.5, 142.6, 128.4, 128.4, 128.3, 128.3, 125.7, 114.4, 46.9, 41.2, 40.7, 38.8, 30.4, 27.6, 27.6, 20.4. ESI-HR calcd for $\mathrm{C}_{18} \mathrm{H}_{28} \mathrm{NO}^{+}\left([\mathrm{M}+\mathrm{H}]^{+}\right)$274.2165, found 274.2158. IR $v\left(\mathrm{~cm}^{-1}\right) 3453,2965,2247$, $1653,1519,1201,1005,730,649 .[\alpha]^{25} \mathrm{D}=+4.5\left(c=1.0, \mathrm{CHCl}_{3}\right)$ for a $92 \%$ ee sample. The enantiomeric purity of this compound was determined by HPLC analysis in comparison with authentic racemic material (Chiralpak ID, hexane $:{ }^{i} \mathrm{PrOH}=99: 1,1.0 \mathrm{~mL} / \mathrm{min}, 210 \mathrm{~nm}$ ): $\mathrm{t}_{\text {major }}=$ $53.933 \mathrm{~min}, \mathrm{t}_{\text {minor }}=61.524 \mathrm{~min}$. 
<smiles>CC[C@](CCBr)(CNC(=O)Br)CN(C)C</smiles>

Amide 5d: 5a ( $0.10 \mathrm{mmol}, 1.0$ equiv) and palladium $10 \%$ on carbon $(9.0 \mathrm{mg})$ were placed in a 10 $\mathrm{mL}$ Schlenk tube. The tube was evacuated and refilled with hydrogen through a hydrogen balloon. After addition of $1.0 \mathrm{~mL}$ of methanol, the mixture was stirred at room temperature for $24 \mathrm{~h}$ under balloon pressure of hydrogen. The reaction mixture was filtered through Celite and concentrated. The crude product was purified directly by column chromatography on silica gel with PE/EtOAc mixture as eluent and $\mathbf{5 d}$ was obtained as a colorless oil (24.8 $\mathrm{mg}, 90 \%$ yield). ${ }^{1} \mathbf{H}$ NMR (400 MHz, $\left.\mathrm{CDCl}_{3}\right) \delta 7.28(\mathrm{ddt}, J=8.9,6.8,1.2 \mathrm{~Hz}, 2 \mathrm{H}), 7.18(\mathrm{ddq}, J=6.9,3.4,1.8,1.3 \mathrm{~Hz}, 3 \mathrm{H}), 5.59(\mathrm{~s}, 1 \mathrm{H})$, $3.19(\mathrm{~d}, J=6.2 \mathrm{~Hz}, 2 \mathrm{H}), 2.57(\mathrm{dd}, J=10.5,6.9 \mathrm{~Hz}, 2 \mathrm{H}), 1.53-1.43(\mathrm{~m}, 2 \mathrm{H}), 1.40-1.25(\mathrm{~m}, 2 \mathrm{H})$, $1.21(\mathrm{~s}, 9 \mathrm{H}), 0.94-0.86(\mathrm{~m}, 6 \mathrm{H}) .{ }^{13} \mathbf{C}$ NMR $\left(101 \mathrm{MHz}, \mathrm{CDCl}_{3}\right) \delta 178.1,143.0,128.4,128.3,125.7$, $46.1,39.4,38.9,36.9,30.1,29.9,27.7,22.5,7.9$. ESI-HR calcd for $\mathrm{C}_{18} \mathrm{H}_{30} \mathrm{NO}^{+}\left([\mathrm{M}+\mathrm{H}]^{+}\right) 276.2322$, found 276.2313. IR $v\left(\mathrm{~cm}^{-1}\right) 3476,3365,2961,2933,2869,1645,1520,1456,1265,1199,733,699$. $[\alpha]^{25} \mathrm{D}=-8.5\left(c=1.0, \mathrm{CHCl}_{3}\right)$ for a $92 \%$ ee sample. The enantiomeric purity of this compound was determined by HPLC analysis in comparison with authentic racemic material (Chiralpak OJH, hexane $\left.:{ }^{i} \mathrm{PrOH}=99: 1,1.0 \mathrm{~mL} / \mathrm{min}, 210 \mathrm{~nm}\right): \mathrm{t}_{\mathrm{major}}=10.828 \mathrm{~min}, \mathrm{t}_{\mathrm{minor}}=14.184 \mathrm{~min}$.<smiles>CC(CBr)(CNC(=O)Br)C(=O)OCc1ccccc1</smiles>

Acid 5e: Amide 5a (0.10 mmol, 1.0 equiv), $\mathrm{NaIO}_{4}(0.40 \mathrm{mmol}, 4$ equiv.) were placed in a $10 \mathrm{~mL}$ flask. $\mathrm{CCl}_{4}(0.20 \mathrm{~mL}), \mathrm{MeCN}(0.20 \mathrm{~mL}), \mathrm{H}_{2} \mathrm{O}(0.30 \mathrm{~mL})$ and $\mathrm{RuCl}_{3}(1.0 \mathrm{mg}, 5 \mathrm{~mol} \%)$ were successively added into the flask. The reaction was stirred at $\mathrm{rt}$ for $2 \mathrm{~h}$. After the completion of the reaction, the reaction mixture was extracted with DCM. The combined organic layers were dried over anhydrous $\mathrm{Na}_{2} \mathrm{SO}_{4}$. After removal of the solvent, the residue was purified by silica gel chromatography and $\mathbf{5 e}$ was obtained as a white solid (12.5 mg, 55\% yield). ${ }^{1} \mathbf{H}$ NMR (400 MHz, $\left.\mathrm{CDCl}_{3}\right) \delta 7.33-7.22(\mathrm{~m}, 2 \mathrm{H}), 7.22-7.12(\mathrm{~m}, 3 \mathrm{H}), 6.39(\mathrm{t}, J=6.3 \mathrm{~Hz}, 1 \mathrm{H}), 3.52(\mathrm{qd}, J=13.8,6.3$ $\mathrm{Hz}, 2 \mathrm{H}), 2.64$ (tt, $J=10.8,7.3 \mathrm{~Hz}, 2 \mathrm{H}), 1.90(\mathrm{~m}, 2 \mathrm{H}), 1.28(\mathrm{~s}, 3 \mathrm{H}), 1.21(\mathrm{~s}, 9 \mathrm{H}) .{ }^{13} \mathbf{C}$ NMR (101 $\left.\mathrm{MHz}, \mathrm{CDCl}_{3}\right) \delta 181.4,179.0,141.6,128.4,128.3,126.0,46.8,44.9,38.8,38.7,30.7,27.5,20.4$. ESI-HR calcd for $\mathrm{C}_{17} \mathrm{H}_{26} \mathrm{NO}_{3}{ }^{+}\left([\mathrm{M}+\mathrm{H}]^{+}\right)$292.1907, found 292.1896. IR $v\left(\mathrm{~cm}^{-1}\right) 3450,2962,1706$, 
$1622,1527,1264,1206,1138,735,701$. M.P. $113-114^{\circ} \mathrm{C} .[\alpha]^{25} \mathrm{D}=-9.2\left(c=1.0, \mathrm{CHCl}_{3}\right)$.<smiles>C[C@](CBr)(CNC(=O)Br)c1cn([13CH3])nn1</smiles>

Triazole 5f: Amide 5a ( $0.10 \mathrm{mmol}, 1.0$ equiv) and copper thiophene-2-carboxylate $(0.020 \mathrm{mmol}$, 0.2 equiv) were added in toluene $(0.60 \mathrm{~mL})$. The reaction mixture was cooled in an ice-water bath. Subsequently, sulfonyl azide $(0.20 \mathrm{mmol}, 2.0$ equiv) was added slowly, and the reaction mixture was allowed to warm to room temperature and stirred until completion as judged by TLC. Saturated aq $\mathrm{NH}_{4} \mathrm{Cl}(10 \mathrm{~mL})$ was added and the mixture was extracted with EtOAc $(2 \times 10 \mathrm{~mL})$, dried over $\mathrm{Na}_{2} \mathrm{SO}_{4}$. After removal of the solvent, the residue was purified by silica gel chromatography and $\mathbf{5 f}$ was obtained as a pale yellow oil (42.8 $\mathrm{mg}, 91 \%$ yield). ${ }^{1} \mathbf{H} \mathbf{~ N M R}\left(400 \mathrm{MHz}, \mathrm{CDCl}_{3}\right) \delta 8.00(\mathrm{~d}, J=$ $8.5 \mathrm{~Hz}, 2 \mathrm{H}), 7.91(\mathrm{~s}, 1 \mathrm{H}), 7.39(\mathrm{~d}, J=8.3 \mathrm{~Hz}, 2 \mathrm{H}), 7.27-7.19(\mathrm{~m}, 2 \mathrm{H}), 7.17-7.11(\mathrm{~m}, 1 \mathrm{H}), 7.10$ $-7.03(\mathrm{~m}, 2 \mathrm{H}), 6.58(\mathrm{t}, J=6.0 \mathrm{~Hz}, 1 \mathrm{H}), 3.73(\mathrm{dd}, J=13.5,7.0 \mathrm{~Hz}, 1 \mathrm{H}), 3.34(\mathrm{dd}, J=13.5,4.9 \mathrm{~Hz}$, 1H), $2.64-2.50$ (m, 1H), 2.45 (s, 3H), 2.37 (ddd, $J=18.3,11.9,5.3 \mathrm{~Hz}, 1 \mathrm{H}), 1.94$ (qdd, $J=13.6$, 11.7, $5.3 \mathrm{~Hz}, 2 \mathrm{H}), 1.37$ (s, 3H), $1.12(\mathrm{~s}, 9 \mathrm{H}) .{ }^{13} \mathbf{C} \mathbf{~ N M R}\left(101 \mathrm{MHz}, \mathrm{CDCl}_{3}\right) \delta$ 178.4, 154.0, 147.4, $141.7,132.9,130.5,128.6,128.3,128.2,125.9,120.1,47.2,40.6,38.7,38.4,30.3,27.5,22.7,21.8$. ESI-HR calcd for $\mathrm{C}_{25} \mathrm{H}_{33} \mathrm{~N}_{4} \mathrm{O}_{3} \mathrm{~S}^{+}\left([\mathrm{M}+\mathrm{H}]^{+}\right) 469.2268$, found 469.2261. IR $v\left(\mathrm{~cm}^{-1}\right) 3380,2964$, $2869,1657,1521,1392,1195,1178,1005,701,674,588,544 .[\alpha]^{25} \mathrm{D}=+6.0\left(c=1.0, \mathrm{CHCl}_{3}\right)$ for a $92 \%$ ee sample. The enantiomeric purity of this compound was determined by HPLC analysis in comparison with authentic racemic material (Chiralpak IE, hexane : ${ }^{i} \mathrm{PrOH}=80: 20,1.0 \mathrm{~mL} / \mathrm{min}$, $210 \mathrm{~nm}): \mathrm{t}_{\text {major }}=24.265 \mathrm{~min}, \mathrm{t}_{\mathrm{minor}}=22.585 \mathrm{~min}$.<smiles>CC(C)(C)C(=O)NC[C@](C)(CBr)c1cc2ccccc2n1[13CH3]</smiles>

Indole 5g: Amide 5a (0.10 mmol, 1.0 equiv), $\mathrm{PdCl}_{2}\left(\mathrm{PPh}_{3}\right)_{2}(0.005 \mathrm{mmol}, 5 \mathrm{~mol} \%)$, $\mathrm{CuI}(0.005$ mmol, $5 \mathrm{~mol} \%)$, N-tosyl-2-iodobenzene $(0.15 \mathrm{mmol}, 1.5$ equiv $)$ were placed in a $10 \mathrm{~mL}$ Schlenk tube. The tube was evacuated and refilled with nitrogen. $\mathrm{DMF}(0.40 \mathrm{~mL})$ and $\mathrm{Et}_{3} \mathrm{~N}(0.20 \mathrm{mmol}, 2.0$ equiv) were successively added into the tube through syringes. The reaction was stirred at $40{ }^{\circ} \mathrm{C}$ for $3 \mathrm{~h}$. After removal of the solvent, the residue was purified by silica gel chromatography and $\mathbf{5 g}$ was obtained as a white solid (37.3 mg, $72 \%$ yield). ${ }^{1} \mathbf{H}$ NMR $\left(400 \mathrm{MHz}, \mathrm{CDCl}_{3}\right) \delta 7.77-7.67(\mathrm{~m}, 2 \mathrm{H})$, 
$7.65(\mathrm{~s}, 1 \mathrm{H}), 7.61-7.54(\mathrm{~m}, 1 \mathrm{H}), 7.35-7.25(\mathrm{~m}, 4 \mathrm{H}), 7.25-7.18(\mathrm{~m}, 3 \mathrm{H}), 7.15(\mathrm{~d}, J=7.9 \mathrm{~Hz}$, 2H), 7.02 (td, $J=7.6,1.2 \mathrm{~Hz}, 1 \mathrm{H}), 5.98(\mathrm{t}, J=6.4 \mathrm{~Hz}, 1 \mathrm{H}), 3.49$ (dd, $J=13.3,6.4 \mathrm{~Hz}, 1 \mathrm{H}), 3.39$ (dd, $J=13.3,6.5 \mathrm{~Hz}, 1 \mathrm{H}), 2.83-2.69(\mathrm{~m}, 2 \mathrm{H}), 2.29$ (s, 3H), 1.83 (ddd, $J=13.4,9.5,7.7 \mathrm{~Hz}, 1 \mathrm{H})$, $1.72(\mathrm{ddd}, J=13.4,9.5,7.8 \mathrm{~Hz}, 1 \mathrm{H}), 1.31(\mathrm{~s}, 3 \mathrm{H}), 1.20(\mathrm{~s}, 9 \mathrm{H}) .{ }^{13} \mathrm{C} \mathbf{N M R}\left(101 \mathrm{MHz}, \mathrm{CDCl}_{3}\right) \delta$ $178.8,143.7,141.6,137.9,136.8,132.1,129.5,129.2,128.5,128.3,127.2,126.0,124.2,120.3$, 114.5, 100.2, 78.1, 47.7, 41.1, 38.9, 37.9, 31.4, 27.6, 24.4, 21.4. ESI-HR calcd for $\mathrm{C}_{31} \mathrm{H}_{37} \mathrm{~N}_{2} \mathrm{O}_{3} \mathrm{~S}^{+}$ $\left([\mathrm{M}+\mathrm{H}]^{+}\right)$517.2519, found 517.2509. IR $v\left(\mathrm{~cm}^{-1}\right) 3422,3020,2967,1650,1522,1493,1290,1214$, 751 , 668. M.P. $139-140^{\circ} \mathrm{C} .[\alpha]^{25} \mathrm{D}=+19.3\left(c=1.0, \mathrm{CHCl}_{3}\right)$ for a $92 \%$ ee sample. The enantiomeric purity of this compound was determined by HPLC analysis in comparison with authentic racemic material (Chiralpak IA, hexane : $\left.{ }^{i} \mathrm{PrOH}=90: 10,1.0 \mathrm{~mL} / \mathrm{min}, 254 \mathrm{~nm}\right): \mathrm{t}_{\text {major }}=14.656 \mathrm{~min}, \mathrm{t}_{\mathrm{minor}}=$ $16.383 \mathrm{~min}$.<smiles>C[C@@](C#CSP)(CN)CBr</smiles>

Amine 5h was prepared according the literature $\operatorname{method}^{5}$. Amide $4 \mathbf{a}(0.10 \mathrm{mmol}, 1.0$ equiv) was dissolved in $2.5 \mathrm{~mL}$ anhydrous DCM under $\mathrm{N}_{2}$. 2-Fluoropyridine ( $0.11 \mathrm{mmol}, 1.1$ equiv) was added and then the mixture was cooled to $-78^{\circ} \mathrm{C}$ and stirred for $5 \mathrm{~min}$. $\mathrm{Tf}_{2} \mathrm{O}(0.11 \mathrm{mmol}, 1.1$ equiv $)$ was added dropwise via syringe and stirred at $-78^{\circ} \mathrm{C}$ for $10 \mathrm{~min}$. The mixture was warmed to $0{ }^{\circ} \mathrm{C}$ and stirred for $10 \mathrm{~min}$. Then EtMgBr solution in THF ( $0.20 \mathrm{mmol}, 2.0$ equiv) was added in one portion at $0{ }^{\circ} \mathrm{C}$ and stirred for $30 \mathrm{~min}$. The mixture was quenched with aqueous $\mathrm{Na}_{2} \mathrm{CO}_{3}$ solution and extracted with EtOAc. It was washed with brine and then concentrated. The residue was dissolved in $1 \mathrm{~mL}$ THF and $3 \mathrm{~mL} 10 \% \mathrm{HCl}$ aqueous solution and then stirred for $1 \mathrm{~h}$ at $\mathrm{rt}$. The mixture was adjusted to $\mathrm{PH} 9-10$ with aqueous $\mathrm{Na}_{2} \mathrm{CO}_{3}$ solution and extracted with $\mathrm{Et}_{2} \mathrm{O}$. The organic phase was washed with water, brine and dried over $\mathrm{Na}_{2} \mathrm{SO}_{4}$. After removal of the solvent, $\mathbf{5} \mathbf{h}$ was obtained as a pale yellow oil $\left(27.5 \mathrm{mg}, 80 \%\right.$ yield). ${ }^{1} \mathbf{H}$ NMR $\left(400 \mathrm{MHz}, \mathrm{CDCl}_{3}\right) \delta 7.29(\mathrm{dd}, J=8.2,6.8 \mathrm{~Hz}$, 2H), $7.19(\mathrm{dd}, J=8.4,7.2 \mathrm{~Hz}, 3 \mathrm{H}), 2.91-2.74(\mathrm{~m}, 2 \mathrm{H}), 2.73-2.54(\mathrm{~m}, 2 \mathrm{H}), 1.87-1.76(\mathrm{~m}, 1 \mathrm{H})$, $1.63-1.53(\mathrm{~m}, 1 \mathrm{H}), 1.36(\mathrm{~s}, 2 \mathrm{H}), 1.24(\mathrm{~s}, 3 \mathrm{H}), 1.17-1.00(\mathrm{~m}, 21 \mathrm{H}) .{ }^{13} \mathbf{C} \mathbf{~ N M R}\left(101 \mathrm{MHz}, \mathrm{CDCl}_{3}\right)$ $\delta 142.6,128.4,128.3,125.7,113.1,82.5,52.7,41.4,39.3,31.7,24.0,18.7,11.2$. ESI-HR calcd for $\mathrm{C}_{22} \mathrm{H}_{38} \mathrm{NSi}^{+}\left([\mathrm{M}+\mathrm{H}]^{+}\right) 344.2768$, found 344.2758. IR $v\left(\mathrm{~cm}^{-1}\right)$ 3027, 2942, 2864, 2160, 1668, 1462 , $1265,883,738,700 .[\alpha]^{25}{ }_{D}=+16.6\left(c=1.0, \mathrm{CHCl}_{3}\right)$ for a $92 \%$ ee sample. The enantiomeric purity 
of this compound was determined by HPLC analysis in comparison with authentic racemic material (Chiralpak IG, hexane : $\left.{ }^{i} \mathrm{PrOH}=99.5: 0.5,1.0 \mathrm{~mL} / \mathrm{min}, 210 \mathrm{~nm}\right): \mathrm{t}_{\text {major }}=13.117 \mathrm{~min}, \mathrm{t}_{\mathrm{minor}}=10.125$ $\min$.<smiles>CC(C)(C)C(=O)N1CCC[C@](C)(C#C[In]S)C1</smiles>

Piperidine 5i: 4q ( $0.050 \mathrm{mmol}, 1.0$ equiv) was dissolved in $2 \mathrm{~mL}$ anhydrous THF, NaH ( $0.10 \mathrm{mmol}$, 2.0 equiv) was added. The mixture was stirred at $60^{\circ} \mathrm{C}$ until completion was indicated by TLC. It was quenched by saturated $\mathrm{NH}_{4} \mathrm{Cl}$ and extracted with EtOAc. The organic phase was washed with water, brine and dried over $\mathrm{Na}_{2} \mathrm{SO}_{4}$. After removal of the solvent, $\mathbf{5 i}$ was obtained as a pale yellow oil $(12.0 \mathrm{mg}, 66 \%$ yield $) . \quad{ }^{1} \mathrm{H}$ NMR $\left(400 \mathrm{MHz}, \mathrm{CDCl}_{3}\right) \delta 4.14(\mathrm{~d}, J=12.8 \mathrm{~Hz}, 1 \mathrm{H}), 4.03(\mathrm{dd}, J=$ 11.3, $6.9 \mathrm{~Hz}, 1 \mathrm{H}), 3.00$ (ddd, $J=13.5,10.3,3.1 \mathrm{~Hz}, 1 \mathrm{H}), 2.83$ (d, $J=12.9 \mathrm{~Hz}, 1 \mathrm{H}), 1.86$ (ddt, $J=$ 17.0, 6.7, 3.3 Hz, 2H), 1.59 (dddd, $J=11.3,6.3,5.0,3.0 \mathrm{~Hz}, 1 \mathrm{H}), 1.43$ (ddd, $J=13.0,11.4,3.7 \mathrm{~Hz}$, 1H), $1.28(\mathrm{~s}, 9 \mathrm{H}), 1.23(\mathrm{~s}, 3 \mathrm{H}), 1.03(\mathrm{~d}, J=4.9 \mathrm{~Hz}, 21 \mathrm{H}) .{ }^{13} \mathbf{C} \mathbf{~ N M R}\left(101 \mathrm{MHz}, \mathrm{CDCl}_{3}\right) \delta 176.4$, 113.0, 81.1, 54.2, 45.9, 38.8, 38.2, 33.3, 28.6, 26.4, 22.7, 18.6, 11.2. ESI-HR calcd for $\mathrm{C}_{22} \mathrm{H}_{42} \mathrm{NOSi}^{+}$ $\left([\mathrm{M}+\mathrm{H}]^{+}\right)$364.3030, found 364.3021. IR $v\left(\mathrm{~cm}^{-1}\right)$ 3019, 2943, 2865, 2400, 1626, 1423, 1214, 748, 668. $[\alpha]^{25} \mathrm{D}=+44\left(c=0.5, \mathrm{CHCl}_{3}\right)$ for an $86 \%$ ee sample. The enantiomeric purity of this compound was determined by HPLC analysis in comparison with authentic racemic material (Chiralpak ODH, hexane $\left.:{ }^{i} \operatorname{PrOH}=99: 1,1.0 \mathrm{~mL} / \mathrm{min}, 210 \mathrm{~nm}\right): \mathrm{t}_{\text {major }}=4.855 \mathrm{~min}$, $\mathrm{t}_{\text {minor }}=5.644 \mathrm{~min}$.

\section{Details of Kinetic Analyses}

\section{General procedure for kinetic measurement using GC}

To a necked tube with magnetic stirrer was added enamide 1a, $\operatorname{Ir}(\mathrm{COD})_{2} \mathrm{OTf},(R)$-DMM-Garphos, DCE, alkyne and dodecane (as internal standard) in glovebox. Then the tube was equipped with rubber stopper and taken outside the glovebox and heated at $60^{\circ} \mathrm{C}$ under a stream of argon. The sample was collected via syringe and pass a short silica gel column with EA as eluent and monitored using GC.

\section{Kinetic isotope effect study}




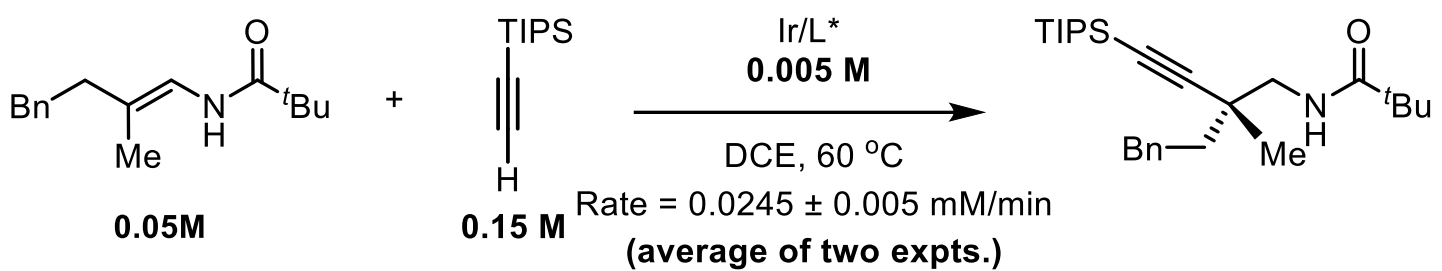

Reaction conditions: enamide $=0.050 \mathrm{M}$, alkyne $=0.15 \mathrm{M}, \mathrm{Ir} / \mathrm{L}^{*}=5.0 \mathrm{mM}, 60^{\circ} \mathrm{C}$. The initial rates were obtained by linear regression of the concentration-time data (between $1 \%$ to $15 \%$ conversion of enamide 1a). The figures for linear regression to obtain the initial rates are shown in Figure S9.
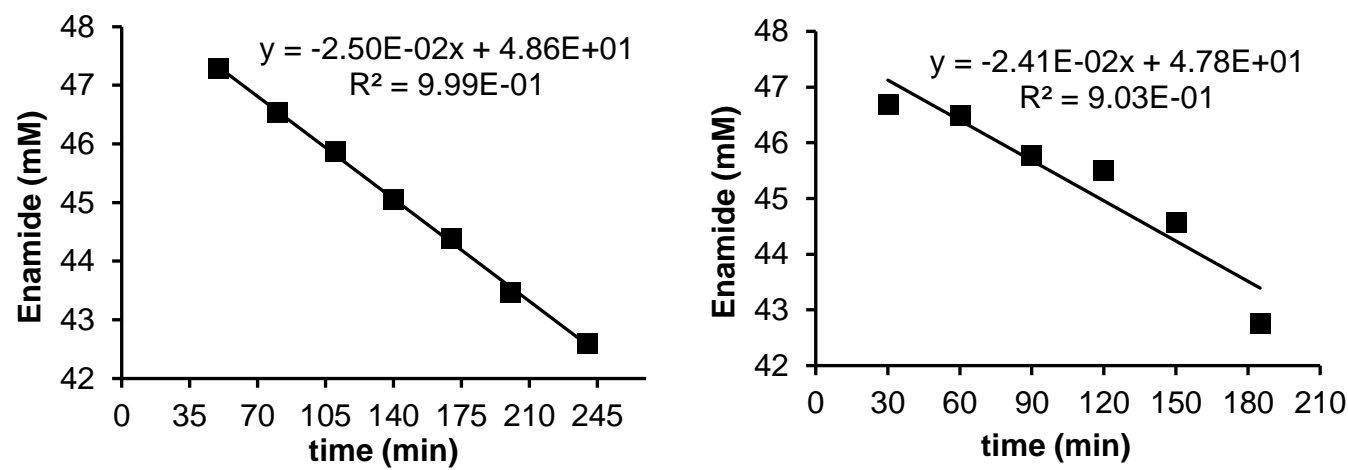

Figure S9. Initial rate of hydroalkynylation of enamide<smiles>[2H]C#C[In-][I-]</smiles>

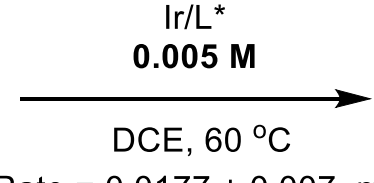

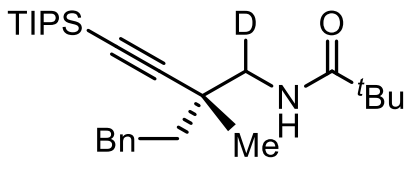

$0.05 \mathrm{M}$

$0.15 \mathrm{M}$

Rate $=0.0177 \pm 0.007 \mathrm{mM} / \mathrm{min}$

(average of two expts.)

$k_{\mathrm{H}} / k_{\mathrm{D}}=1.38$

Reaction conditions: enamide $=0.050 \mathrm{M}, \mathrm{D}$-alkyne $=0.15 \mathrm{M}, \mathrm{Ir} / \mathrm{L}^{*}=5.0 \mathrm{mM}, 60^{\circ} \mathrm{C}$. The initial rates were obtained by linear regression of the concentration-time data ( $1 \%$ to $15 \%$ conversion of enamide 1a). The figures for linear regression to obtain the initial rates are shown in Figure S10.
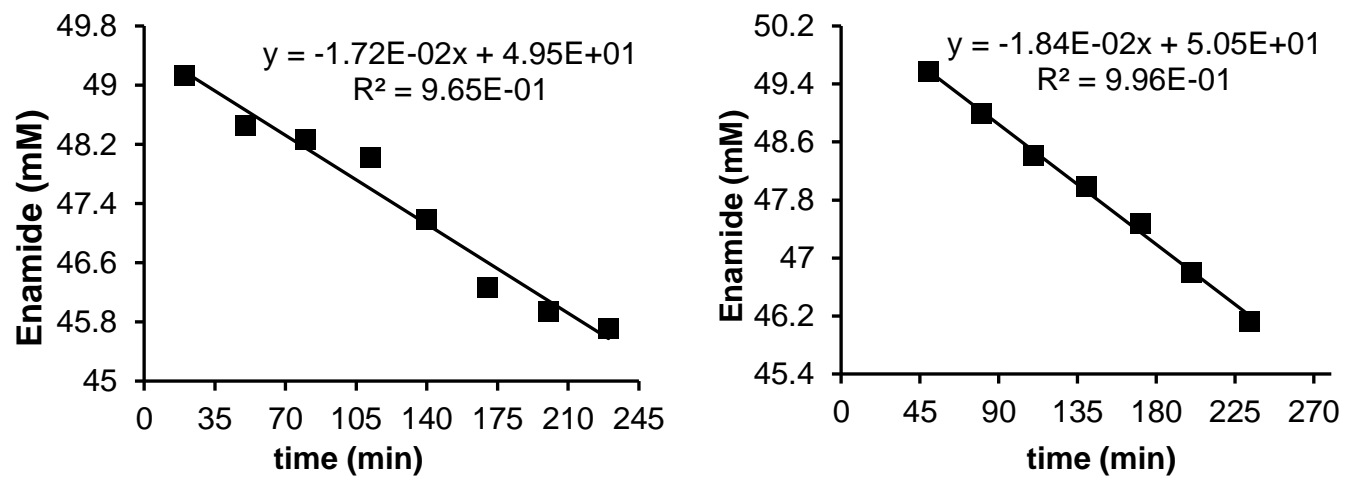

Figure S10. Initial rate of hydroalkynylation of enamide 


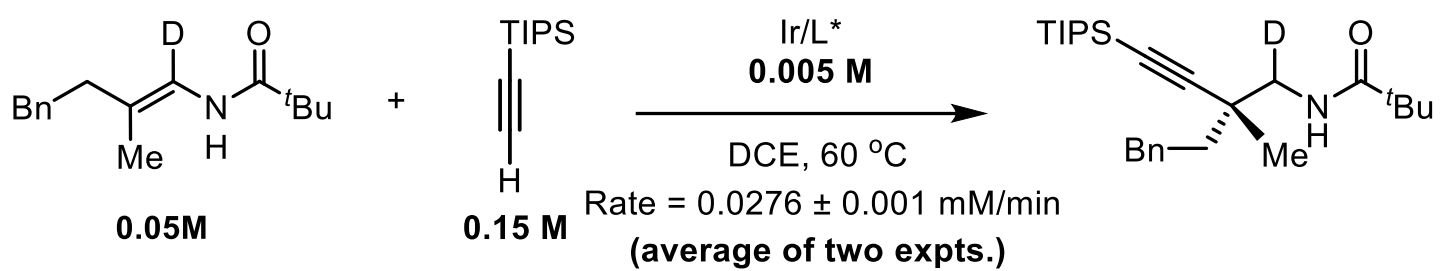

$$
k_{\mathrm{H}} / k_{\mathrm{D}}=0.90
$$

Reaction conditions: D-enamide $=0.050 \mathrm{M}$, alkyne $=0.15 \mathrm{M}, \mathrm{Ir} / \mathrm{L}^{*}=5.0 \mathrm{mM}, 60^{\circ} \mathrm{C}$. The initial rates were obtained by linear regression of the concentration-time data (between $1 \%$ to $15 \%$ conversion of $\mathrm{D}$-enamide 1a). The figures for linear regression to obtain the initial rates are shown in Figure S11.
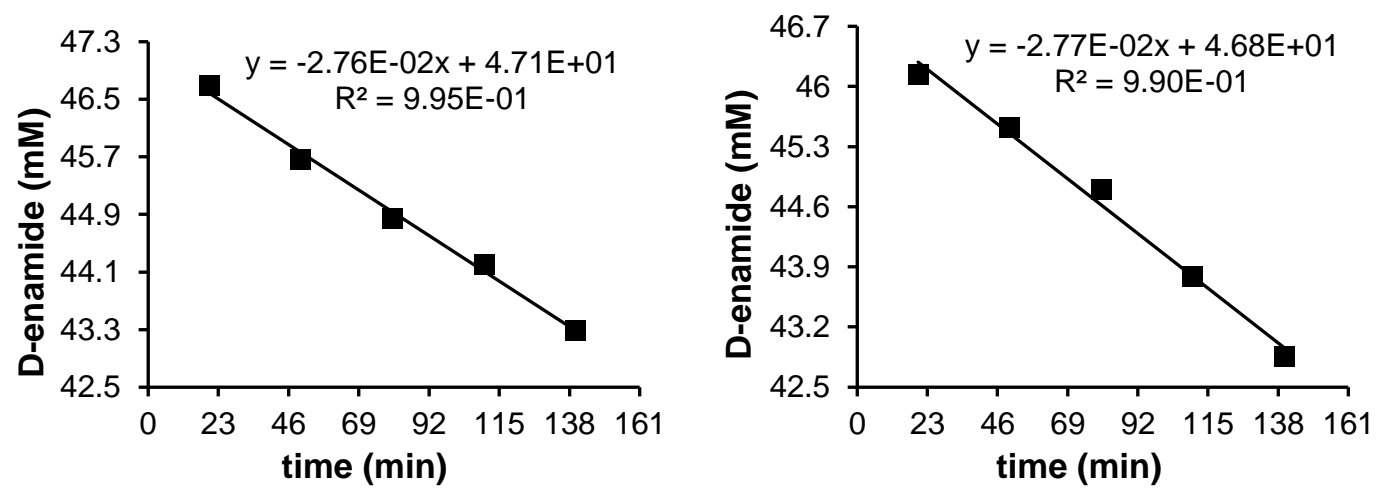

Figure S11. Initial rate of hydroalkynylation of D-enamide<smiles>[R9]/C(NC(=O)Cc1ccccc1)=C(\[2H])CBr</smiles>

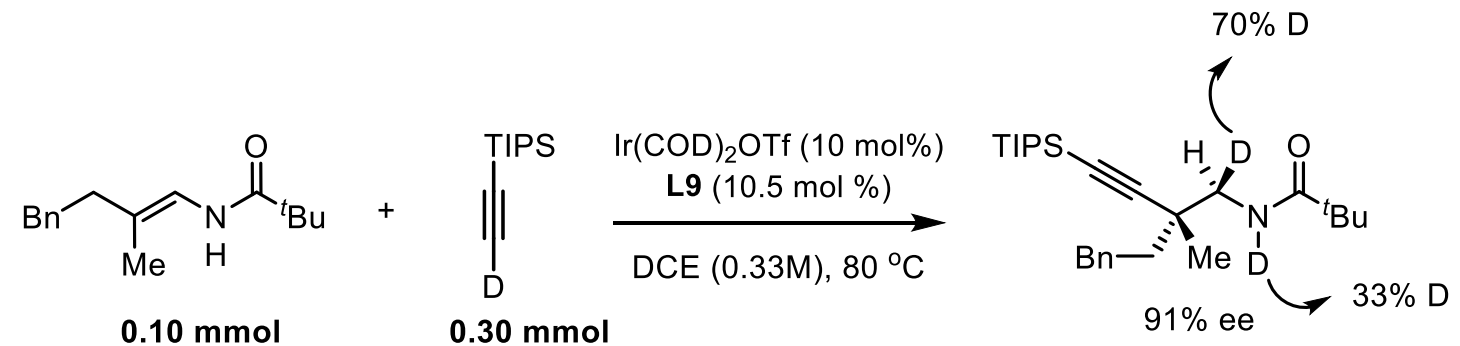

The reactions were conducted according to the general procedure for the synthesis of homopropargyl amides. The deuterium content was analyzed by ${ }^{1} \mathrm{H}$ NMR.

\section{Control experiments for $\mathrm{E} / \mathrm{Z}$ isomerization versus enantioselectivity:}

In an $\mathrm{N}_{2}$-filled glovebox, to a necked tube with magnetic stirrer was added enamide 1a-Z $(0.30$ 
mmol, 1.0 equiv), $\operatorname{Ir}(\mathrm{COD})_{2} \mathrm{OTf}(0.030 \mathrm{mmol}, 10 \mathrm{~mol} \%),(R)$-DMM-Garphos (0.0315 mmol, 10.5 mol \%), DCE (0.9 mL, 0.33M), alkyne (0.90 mmol, 3.0 equiv). Dodecane was added as internal standard. Then the tube was capped with a rubber stopper and taken outside the glovebox and heated at $80{ }^{\circ} \mathrm{C}$ under a stream of argon. At different time, a portion of reaction mixture was taken out via syringe under a stream of argon. The $E / Z$ ratio of remaining enamide and the yield was obtained by GC. The ee of product was obtained by HPLC.

\section{Hydroalkynylation of $Z$-enamide}<smiles>CC(CBr)CBr</smiles>

2

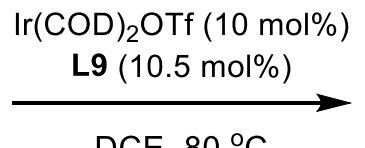

DCE, $80^{\circ} \mathrm{C}$
$\mathrm{Bn}$<smiles>C[C@@H](CBr)CC#C[I-]S</smiles>

3

$\alpha$-Alkynylation<smiles>CC(C)(C)C(=O)NC[C@](C)(C#CS[18F])CBr</smiles>

ent-4a $\beta$-Alkynylation

Table S3. Analysis data in Hydroalkynylation of $Z$-enamide

\begin{tabular}{ccc}
\hline Time (h) & $\mathbf{Z}: \mathbf{E}$ & ee of ent-4a \\
\hline 3 & $49: 1$ & $92 \%$ \\
7 & $49: 1$ & $92 \%$ \\
10 & $49: 1$ & $92 \%$ \\
18 & $49: 1$ & $92 \%$ \\
24 & $49: 1$ & $92 \%$ \\
\hline
\end{tabular}

\section{Identification of Resting State:}

\section{Synthesis and characterization of complex:}

In an $\mathrm{N}_{2}$-filled glovebox, $\operatorname{Ir}(\mathrm{COD})_{2} \mathrm{OTf}(0.10 \mathrm{mmol}, 1.0$ equiv), $(R)$-DMM-Garphos (0.10 mmol, 1.0 equiv) were weighed into a schlenk tube. Anhydrous DCM (3.0 mL) was added via syringe. After $0.5 \mathrm{~h}$, the reaction mixture was concentrated in vacuum. Recrystallized from DCM/hexane gave the titled complex. ${ }^{31} \mathbf{P}$ NMR $\left(162 \mathrm{MHz}, \mathrm{CDCl}_{3}\right) \delta 13.6 .{ }^{1} \mathbf{H}$ NMR $\left(400 \mathrm{MHz}, \mathrm{CDCl}_{3}\right) \delta 7.10$ $(\mathrm{t}, \mathrm{J}=9.5 \mathrm{~Hz}, 8 \mathrm{H}), 6.73-6.65(\mathrm{~m}, 2 \mathrm{H}), 5.96(\mathrm{~d}, \mathrm{~J}=2.2 \mathrm{~Hz}, 2 \mathrm{H}), 4.32(\mathrm{~d}, \mathrm{~J}=7.7 \mathrm{~Hz}, 2 \mathrm{H}), 4.15(\mathrm{q}$, $\mathrm{J}=6.1,5.7 \mathrm{~Hz}, 2 \mathrm{H}), 3.84-3.66(\mathrm{~m}, 18 \mathrm{H}), 3.36(\mathrm{~s}, 6 \mathrm{H}), 2.30(\mathrm{~s}, 14 \mathrm{H}), 2.16(\mathrm{~s}, 16 \mathrm{H}), 1.96(\mathrm{td}, \mathrm{J}=$ 12.0, 11.5, 6.0 Hz, 2H). 


\section{References}

1. Tsuchikama, K.; Kasagawa, M.; Endo, K.; Shibata, T. Org. Lett. 2009, 11, 1821.

2. Zhang, W.-W.; Zhang, S.-L.; Li, B.-J. Angew. Chem. Int. Ed. 2020, 59, 6874.

3. Mousseau, J. J.; Bull, J. A.; Charette, A. B. Angew. Chem. Int. Ed. 2010, 49, 1115.

4. Zheng, J.-S.; Chang, H.-N.; Wang, F.-L.; Liu, L. J. Am. Chem. Soc. 2011, 133, 11080.

5. Bechara, W. S.; Pelletier, G.; Charette, A. B. Nat. Chem., 2012, 4, 228.

6. Bai, X.-Y.; Zhao, W.; Sun, X.; Li, B.-J. J. Am. Chem. Soc. 2019, 141, 19870. 
12. Copies of NMR spectra

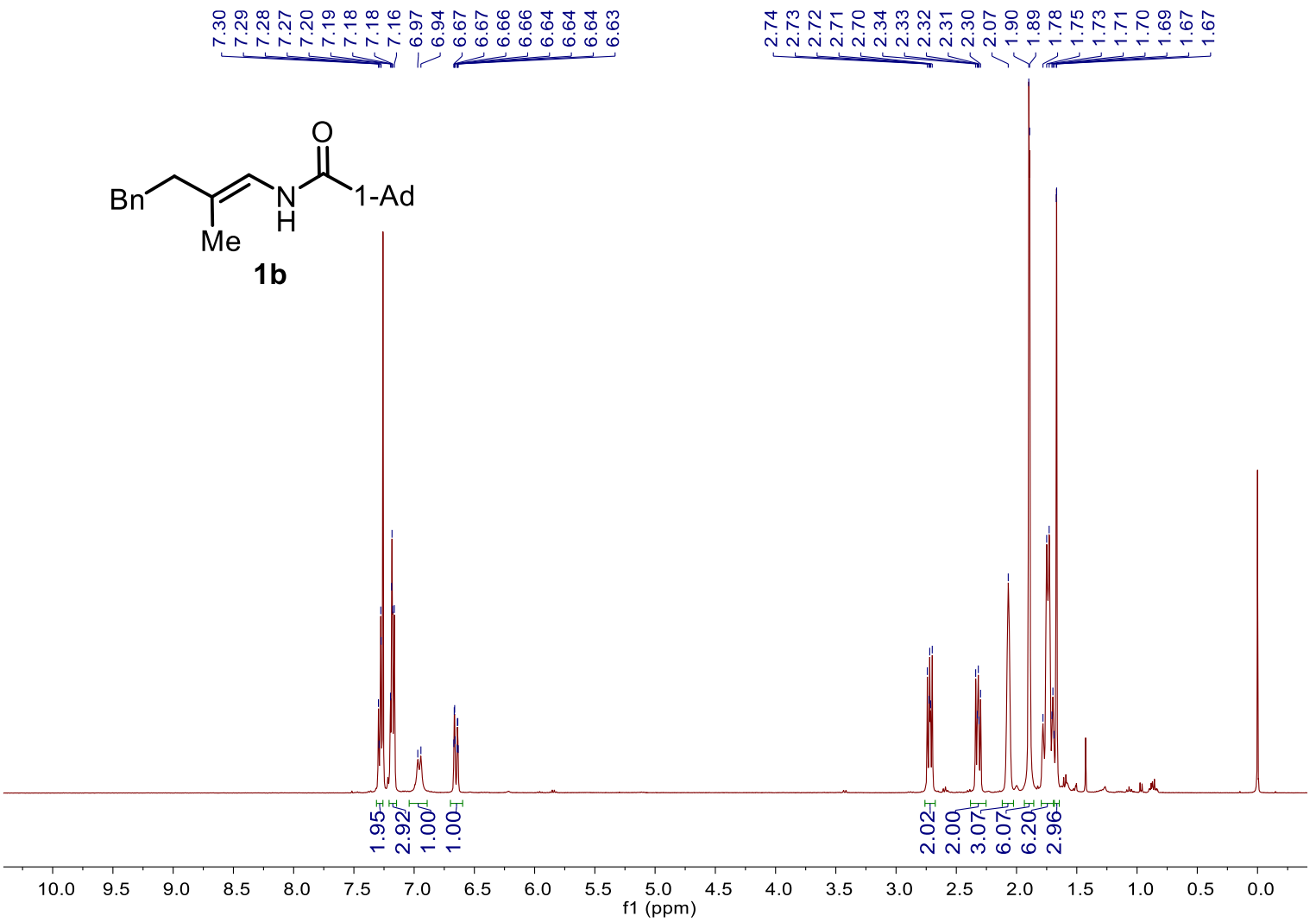

Figure S12. ${ }^{1} \mathbf{H}$ NMR $\left(400 \mathrm{MHz}, \mathrm{CDCl}_{3}\right)$ of $\mathbf{1 b}$<smiles>CC(=CNC(=O)[AlH2])CBr</smiles>

$1 b$

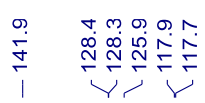

it

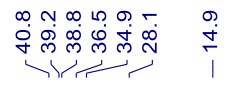
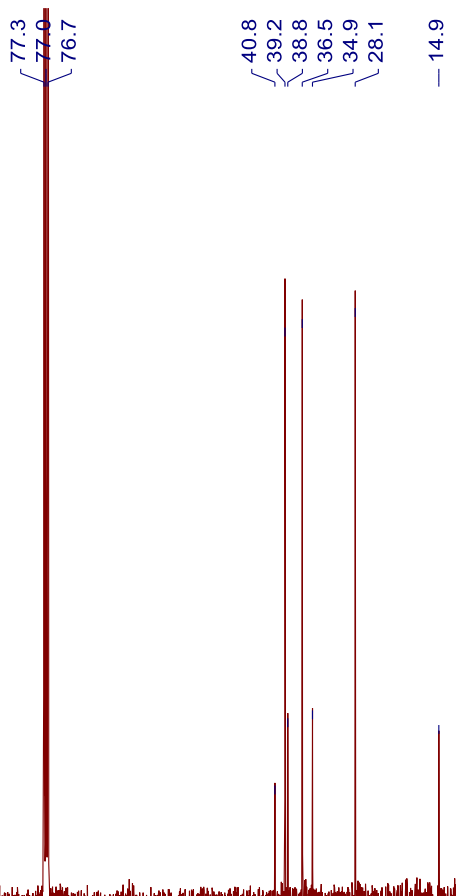

$\begin{array}{lllllllllllll}210 & 200 & 190 & 180 & 170 & 160 & 150 & 140 & 130 & 120 & 110 & 100 & 90\end{array}$

Figure S13. ${ }^{13} \mathbf{C}$ NMR $\left(101 \mathrm{MHz}, \mathrm{CDCl}_{3}\right)$ of $\mathbf{1 b}$ 


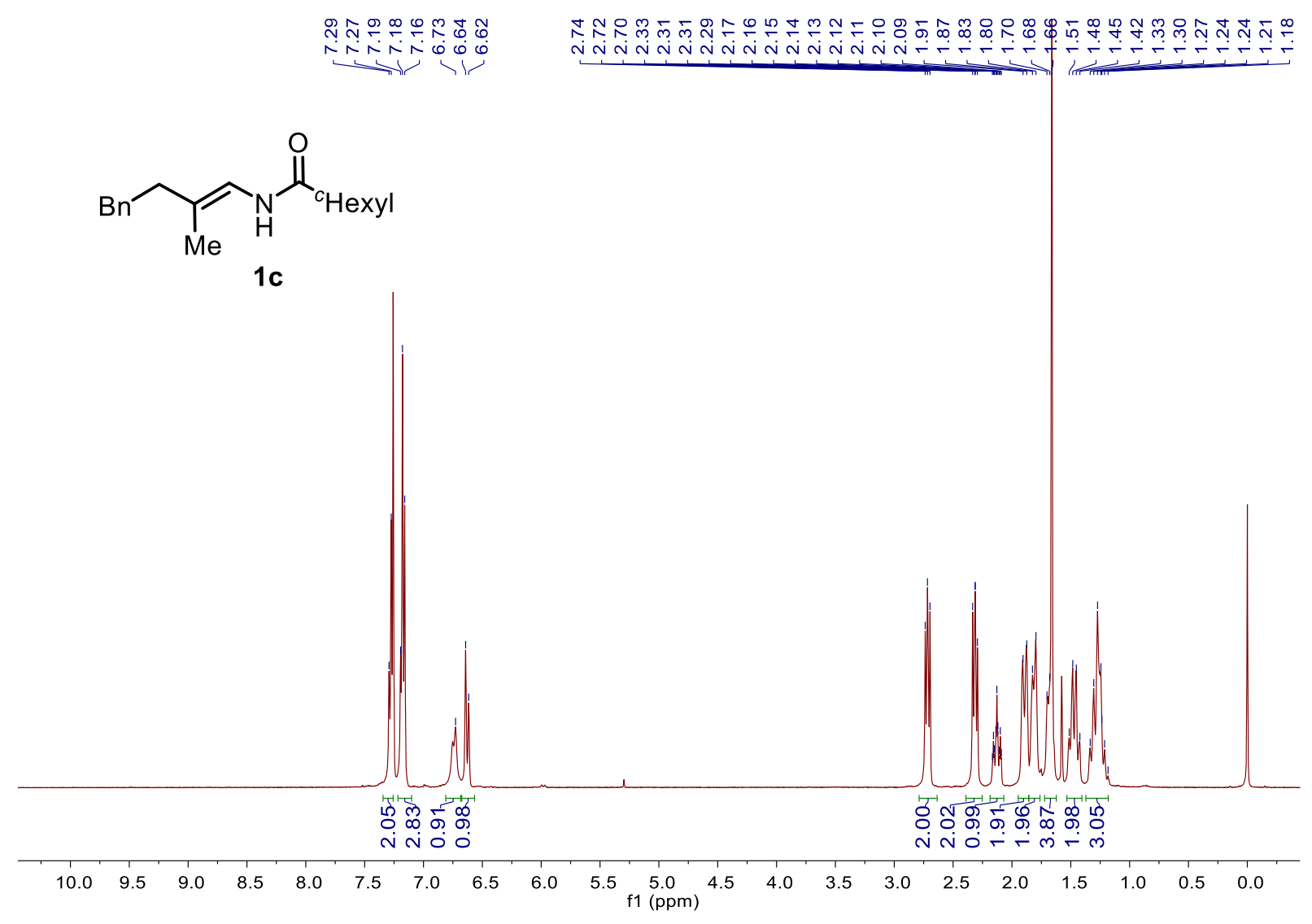

Figure S14. ${ }^{\mathbf{1}} \mathbf{H}$ NMR $\left(400 \mathrm{MHz}, \mathrm{CDCl}_{3}\right)$ of $\mathbf{1 c}$

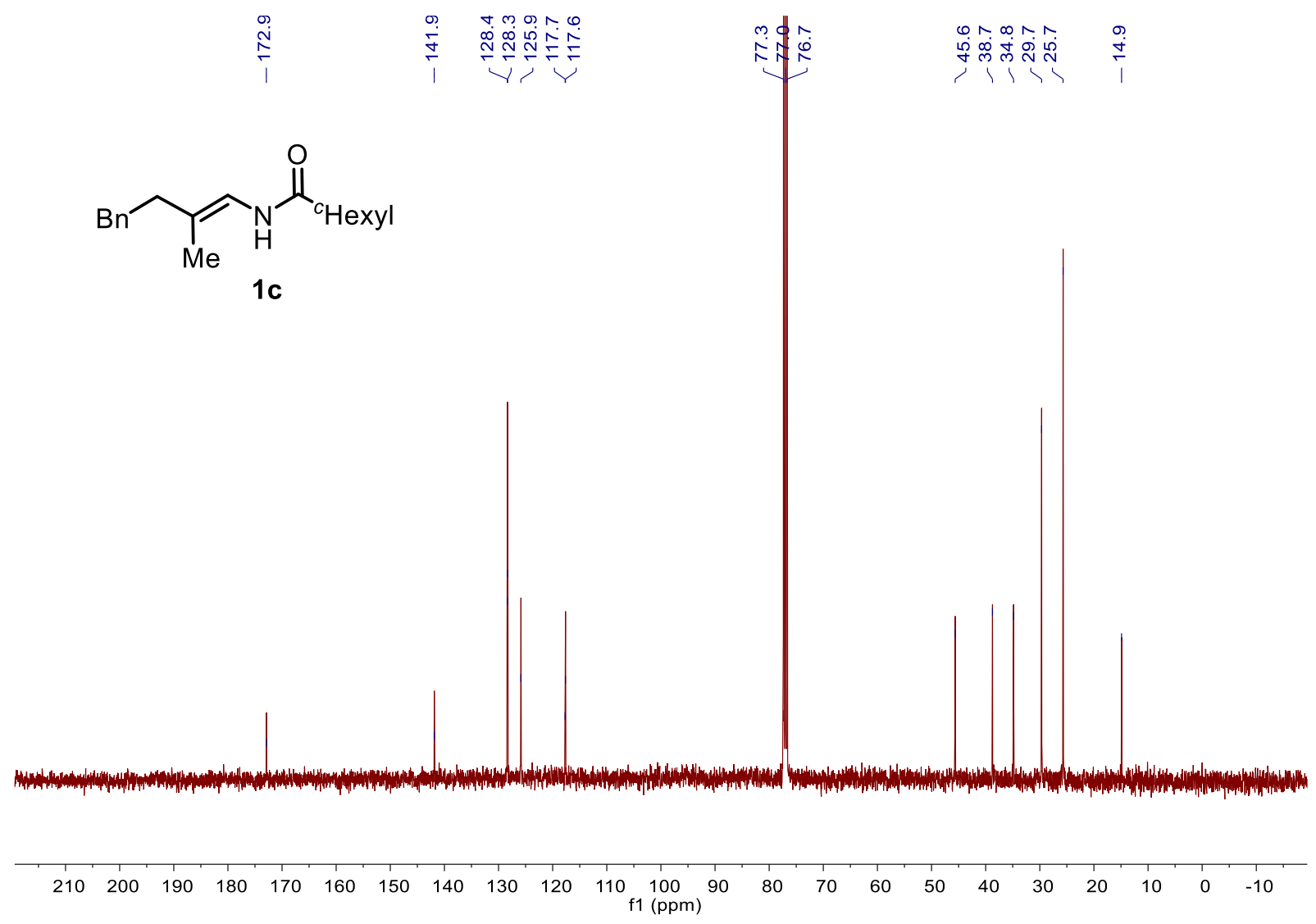

Figure S15. ${ }^{13} \mathbf{C}$ NMR $\left(101 \mathrm{MHz}, \mathrm{CDCl}_{3}\right)$ of 1c 


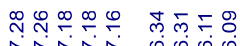

垈证

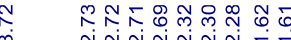

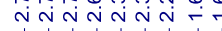

$\overbrace{\mathrm{Me}}^{\mathrm{H}} \overbrace{\mathrm{H}}^{\mathrm{O}}$

$1 \mathrm{~g}$

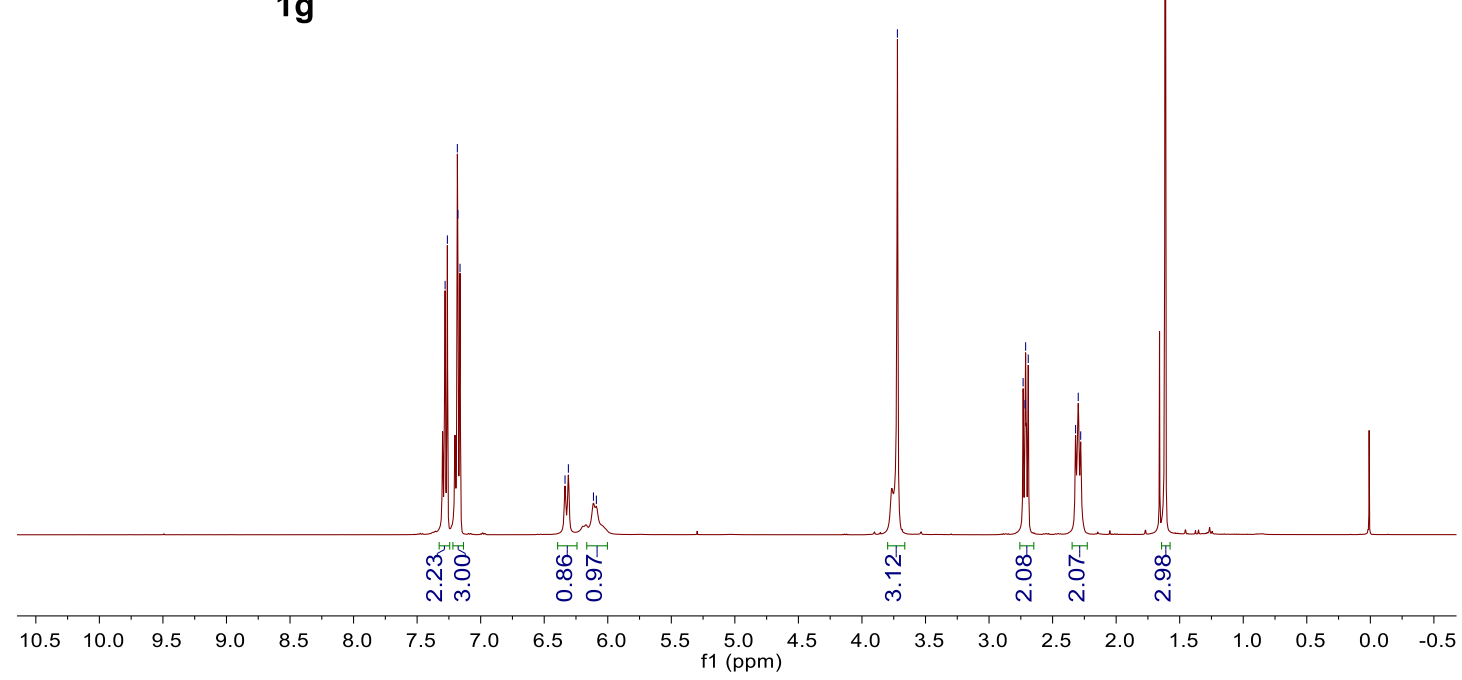

Figure S16. ${ }^{1} \mathbf{H}$ NMR $\left(400 \mathrm{MHz}, \mathrm{CDCl}_{3}\right)$ of $\mathbf{1 g}$

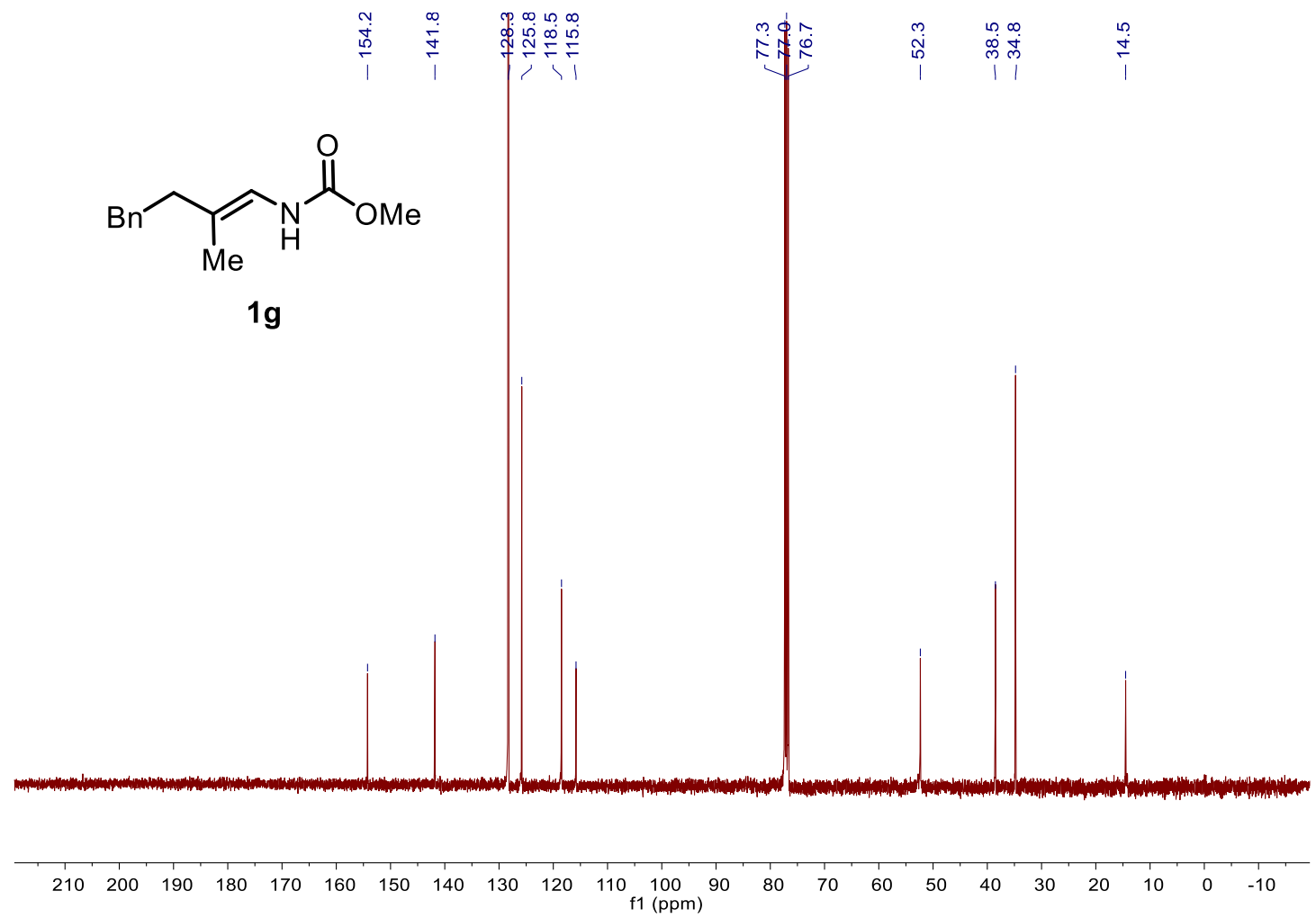

Figure S17. ${ }^{13} \mathbf{C}$ NMR $\left(101 \mathrm{MHz}, \mathrm{CDCl}_{3}\right)$ of $\mathbf{1 g}$ 


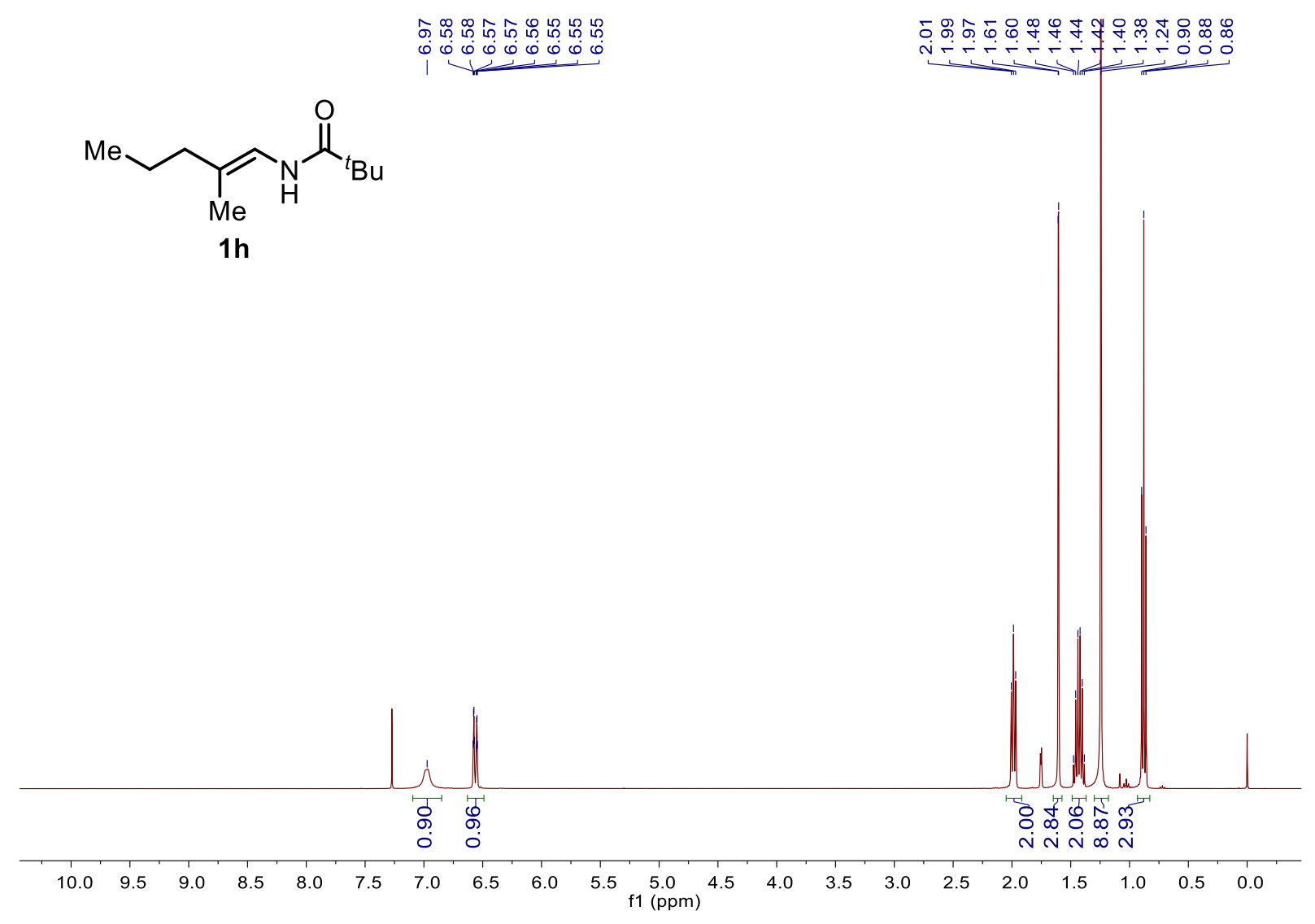

Figure S18. ${ }^{\mathbf{1}} \mathbf{H}$ NMR $\left(400 \mathrm{MHz}, \mathrm{CDCl}_{3}\right)$ of $\mathbf{1 h}$

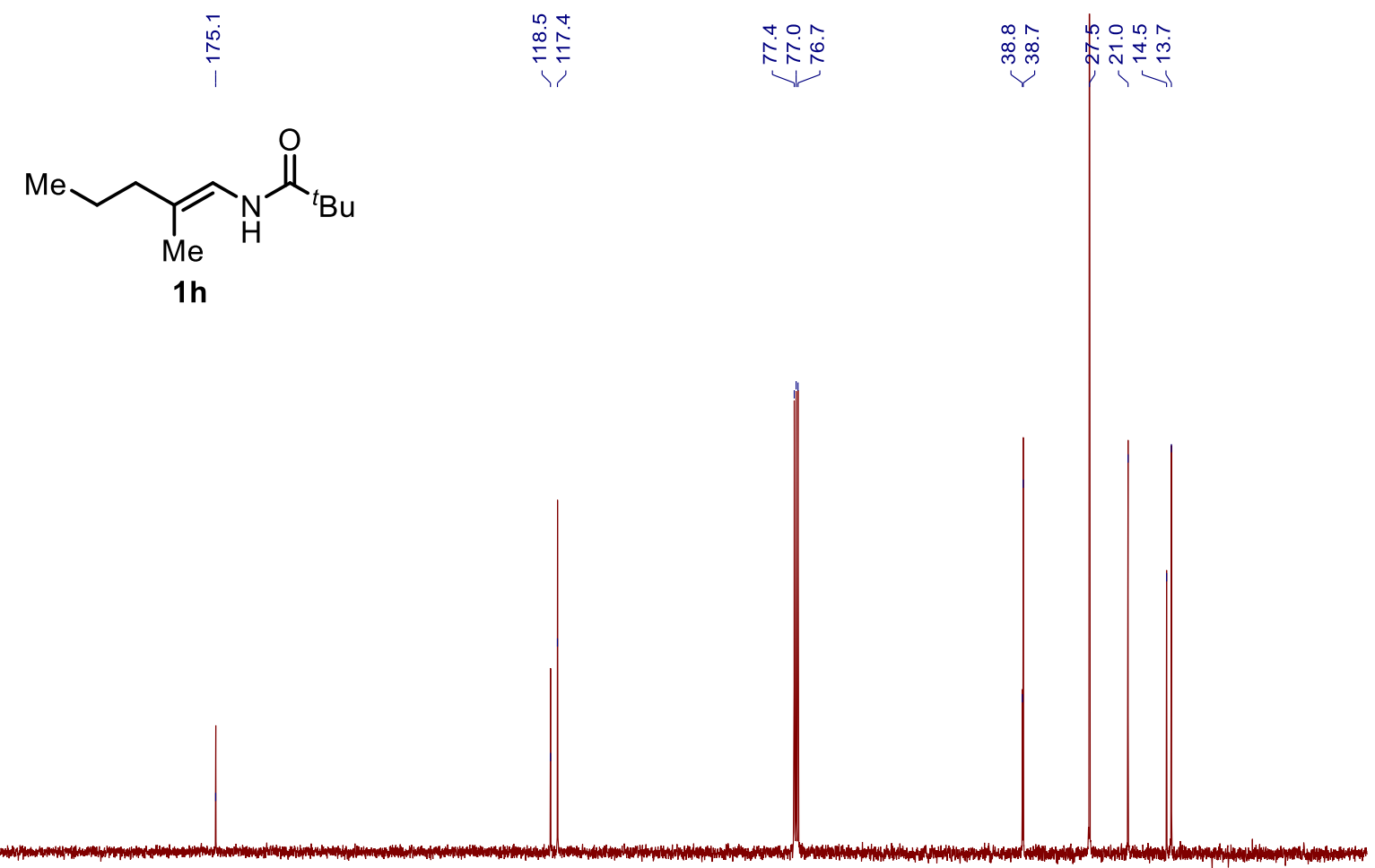

$\begin{array}{lllllllllllllllllllllllllllll}210 & 200 & 190 & 180 & 170 & 160 & 150 & 140 & 130 & 120 & 110 & 100 & 90 & 80 & 70 & 60 & 50 & 40 & 30 & 20 & 10 & 0 & -10\end{array}$

Figure S19. ${ }^{13} \mathbf{C}$ NMR $\left(101 \mathrm{MHz}, \mathrm{CDCl}_{3}\right)$ of $\mathbf{1 h}$ 
<smiles>C/C(=C\NC(=O)CCC(C)C)CCC(C)C</smiles>

Figure S20. ${ }^{1} \mathbf{H}$ NMR $\left(400 \mathrm{MHz}, \mathrm{CDCl}_{3}\right)$ of $\mathbf{1 i}$<smiles>CCCCCC(=CNC(=O)Br)CCCC</smiles>

Figure S21. ${ }^{13} \mathbf{C}$ NMR $\left(101 \mathrm{MHz}, \mathrm{CDCl}_{3}\right)$ of $\mathbf{1 i}$ 


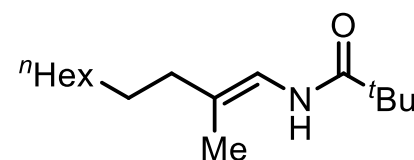

$1 \mathrm{j}$
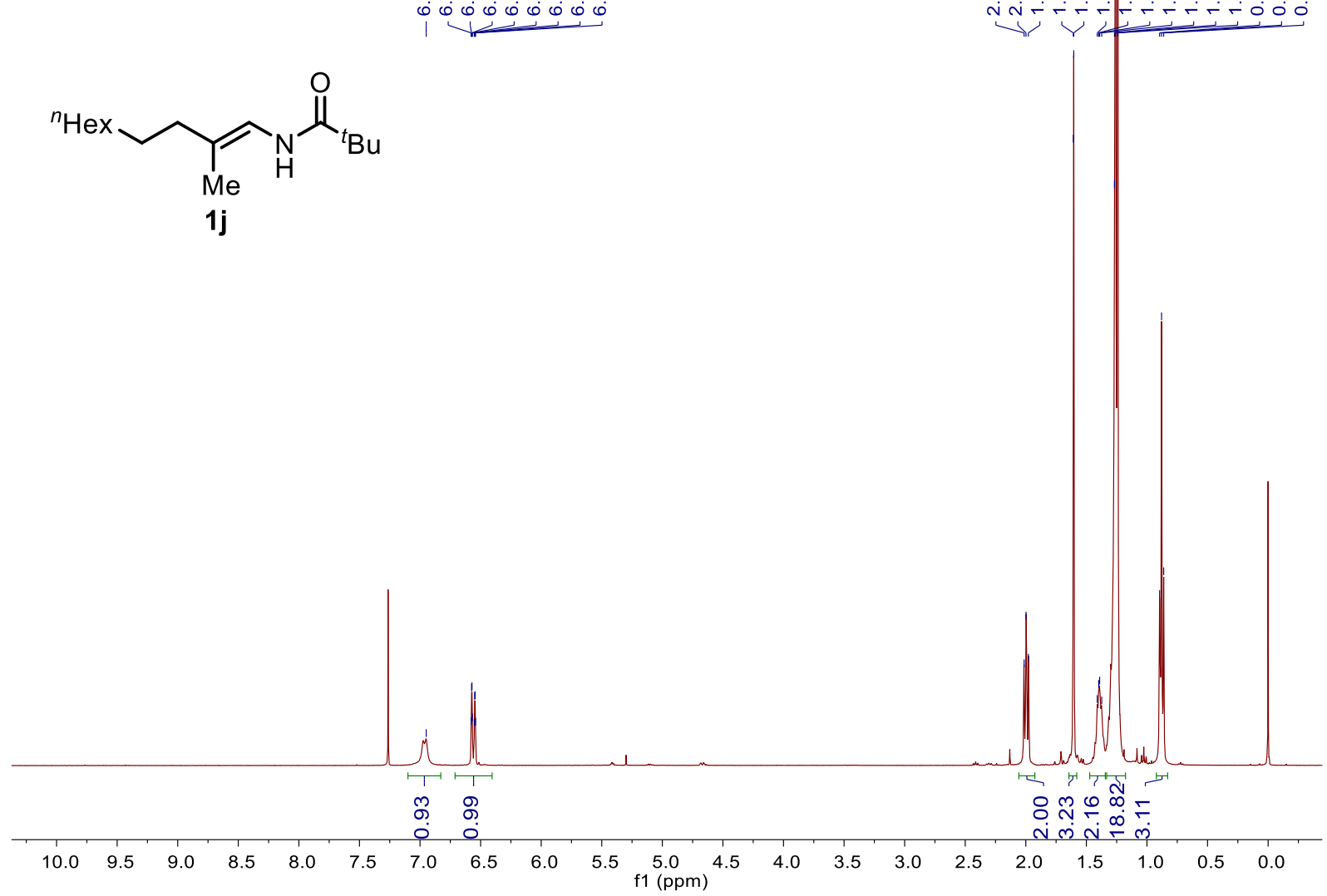

Figure S22. ${ }^{\mathbf{1}} \mathbf{H}$ NMR $\left(400 \mathrm{MHz}, \mathrm{CDCl}_{3}\right)$ of $\mathbf{1 j}$<smiles>CCCCCCCCCCC(C)=CNC(=O)CCC(C)(C)C</smiles>

mog 苑药

$1 \mathrm{j}$

$\begin{array}{llllllllllll}210 & 200 & 190 & 180 & 170 & 160 & 150 & 140 & 130 & 120 & 110 & 100\end{array}$

Figure S23. ${ }^{13} \mathbf{C}$ NMR $\left(101 \mathrm{MHz}, \mathrm{CDCl}_{3}\right)$ of $\mathbf{1 j}$ 


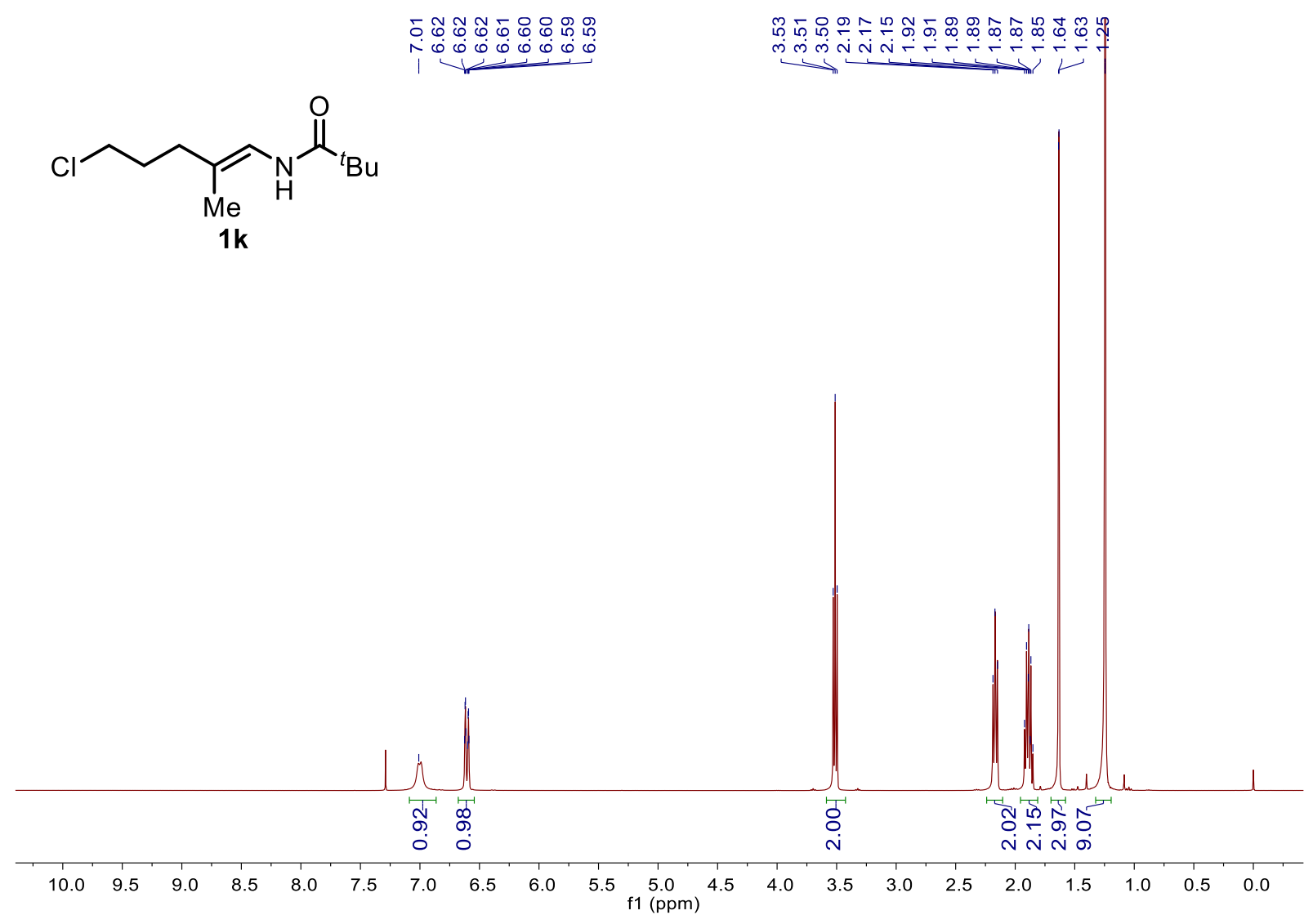

Figure S24. ${ }^{\mathbf{1}} \mathbf{H}$ NMR $\left(400 \mathrm{MHz}, \mathrm{CDCl}_{3}\right)$ of $\mathbf{1 k}$

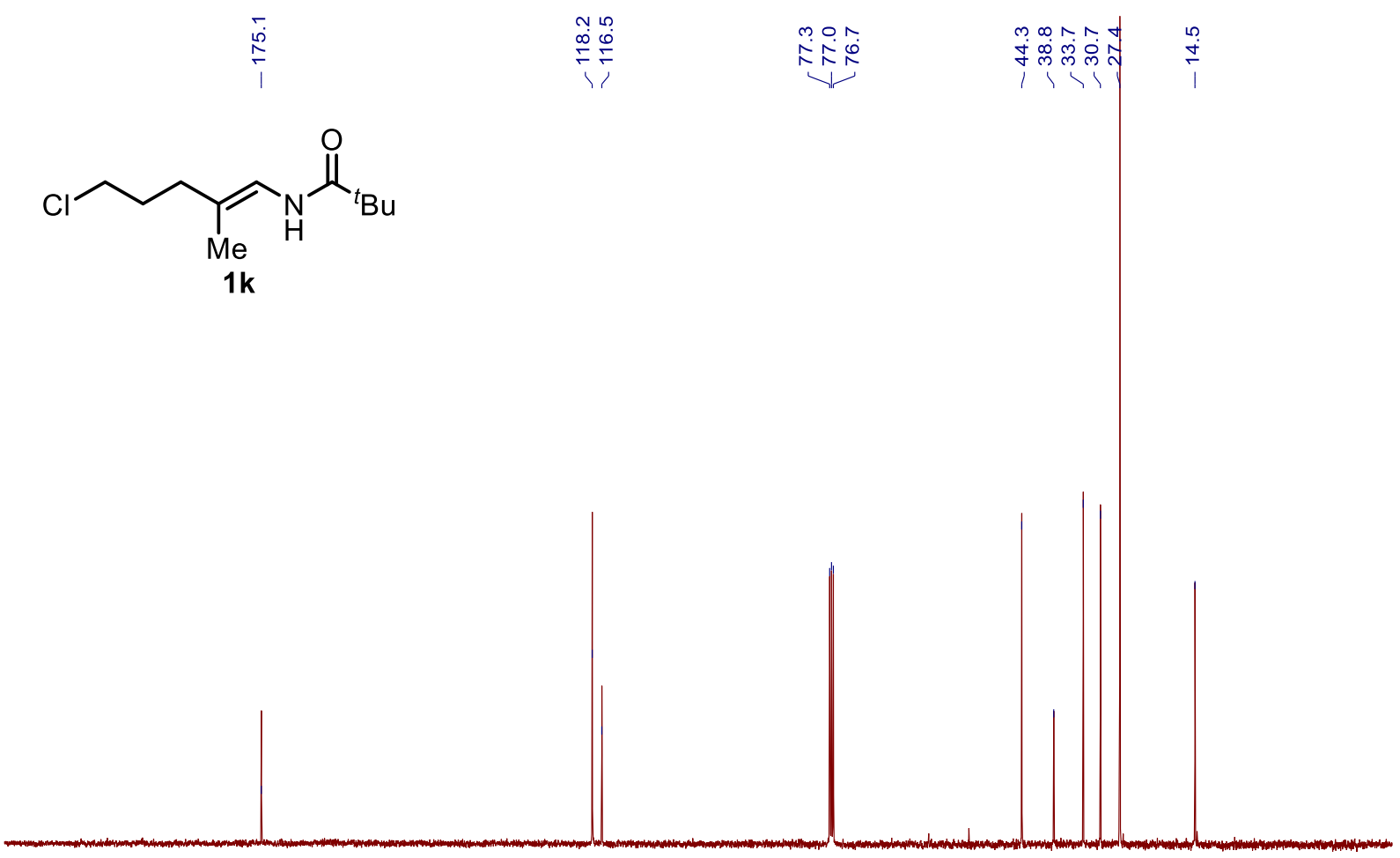

$\begin{array}{lllllllllllllllllllllllllllll}210 & 200 & 190 & 180 & 170 & 160 & 150 & 140 & 130 & 120 & 110 & 100 & 90 & 80 & 70 & 60 & 50 & 40 & 30 & 20 & 10 & 0 & -10\end{array}$

Figure S25. ${ }^{13} \mathbf{C}$ NMR $\left(101 \mathrm{MHz}, \mathrm{CDCl}_{3}\right)$ of $\mathbf{1 k}$ 


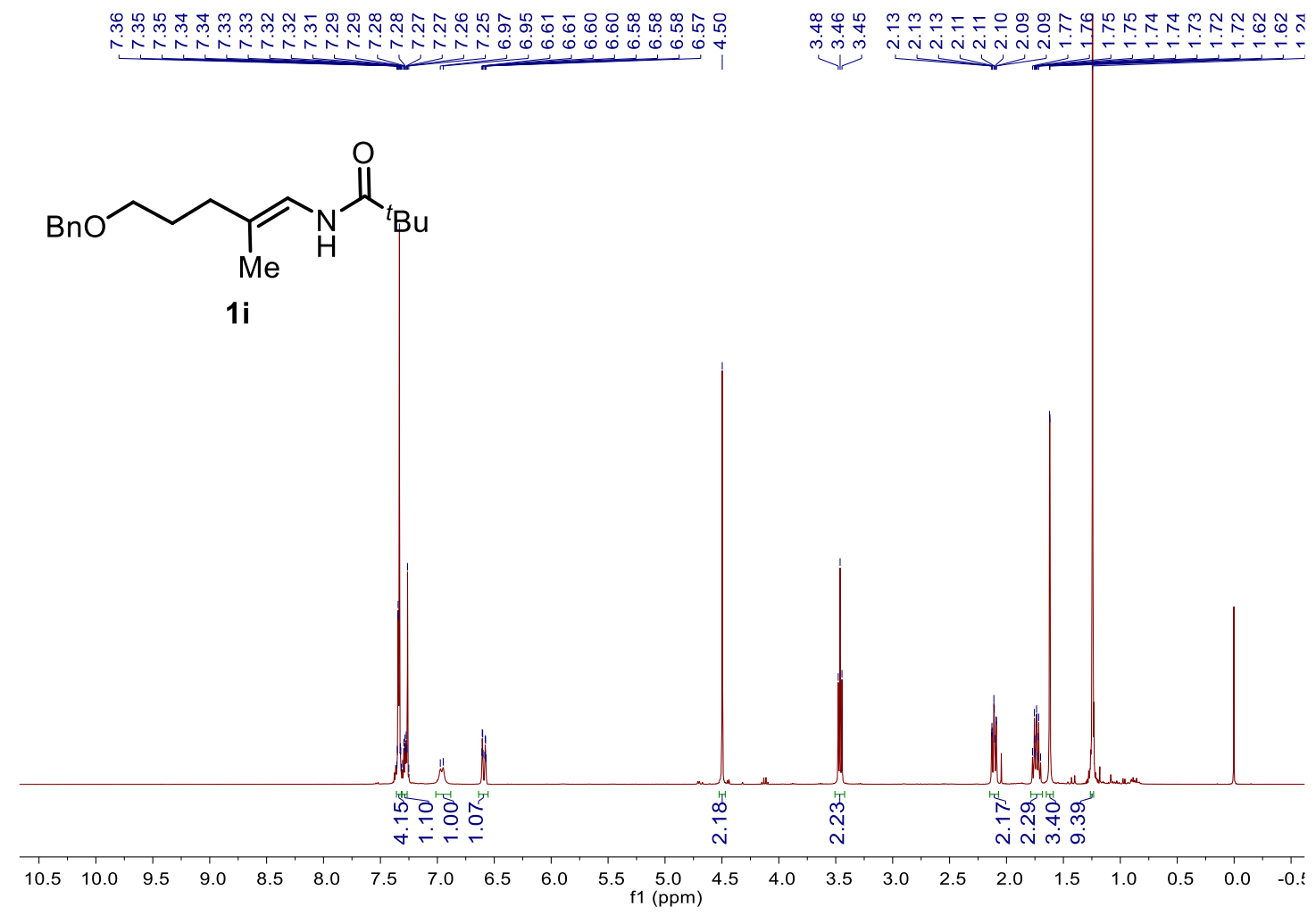

Figure S26. ${ }^{\mathbf{H}} \mathbf{H}$ NMR $\left(400 \mathrm{MHz}, \mathrm{CDCl}_{3}\right)$ of $\mathbf{1 i}$

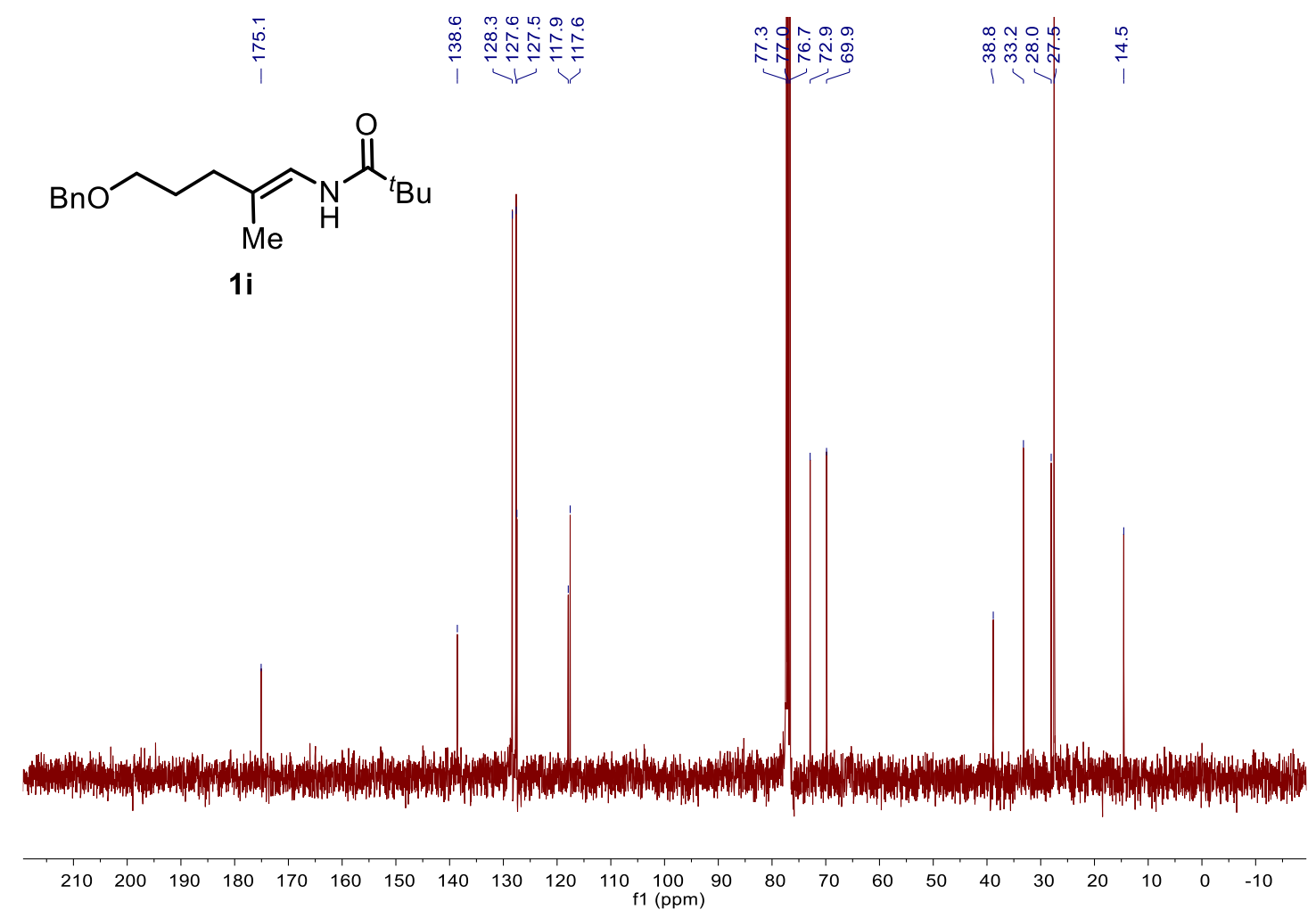

Figure S27. ${ }^{13} \mathbf{C}$ NMR $\left(101 \mathrm{MHz}, \mathrm{CDCl}_{3}\right)$ of $\mathbf{1 i}$ 


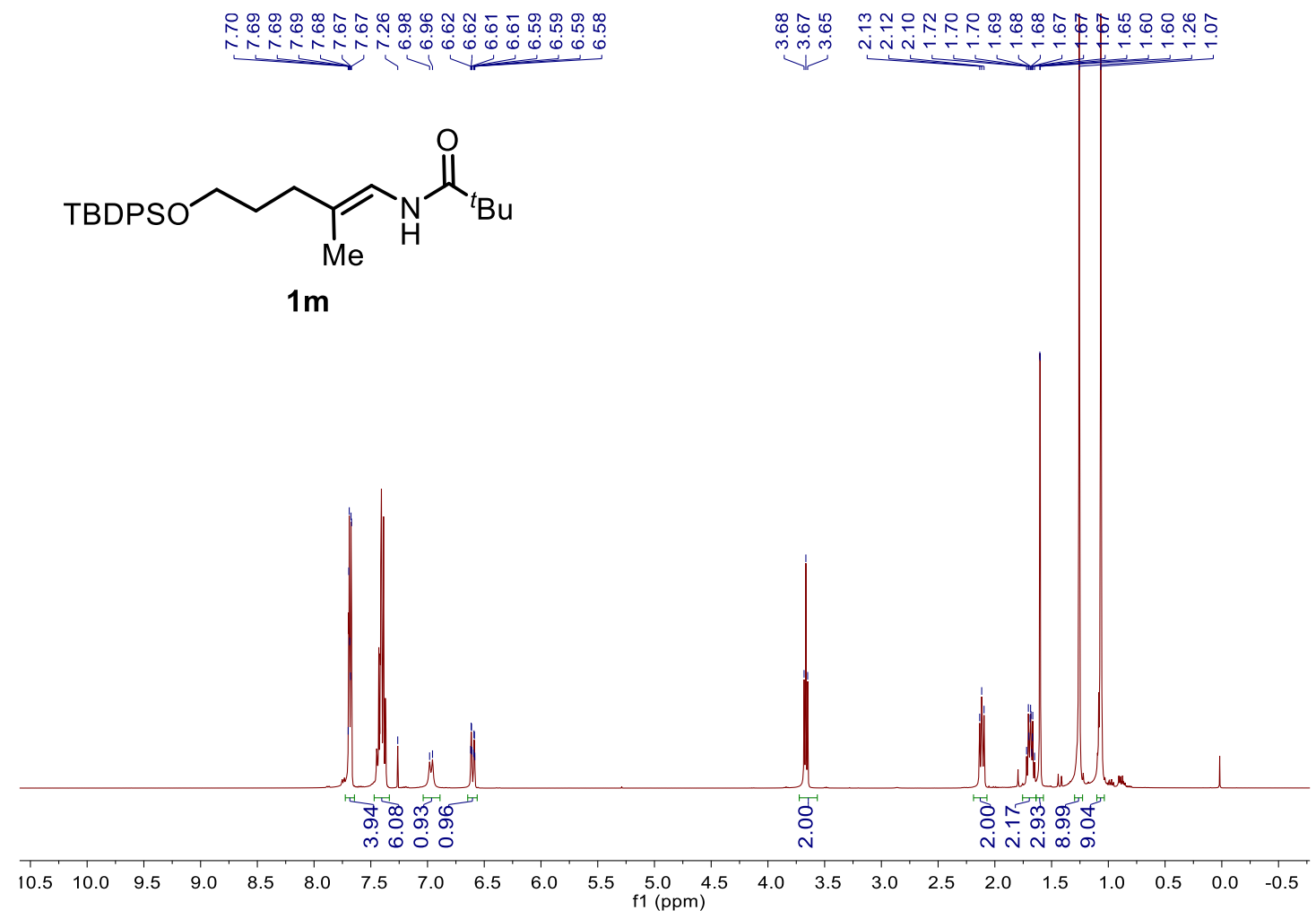

Figure S28. ${ }^{1} \mathbf{H}$ NMR $\left(400 \mathrm{MHz}, \mathrm{CDCl}_{3}\right)$ of $\mathbf{1 m}$

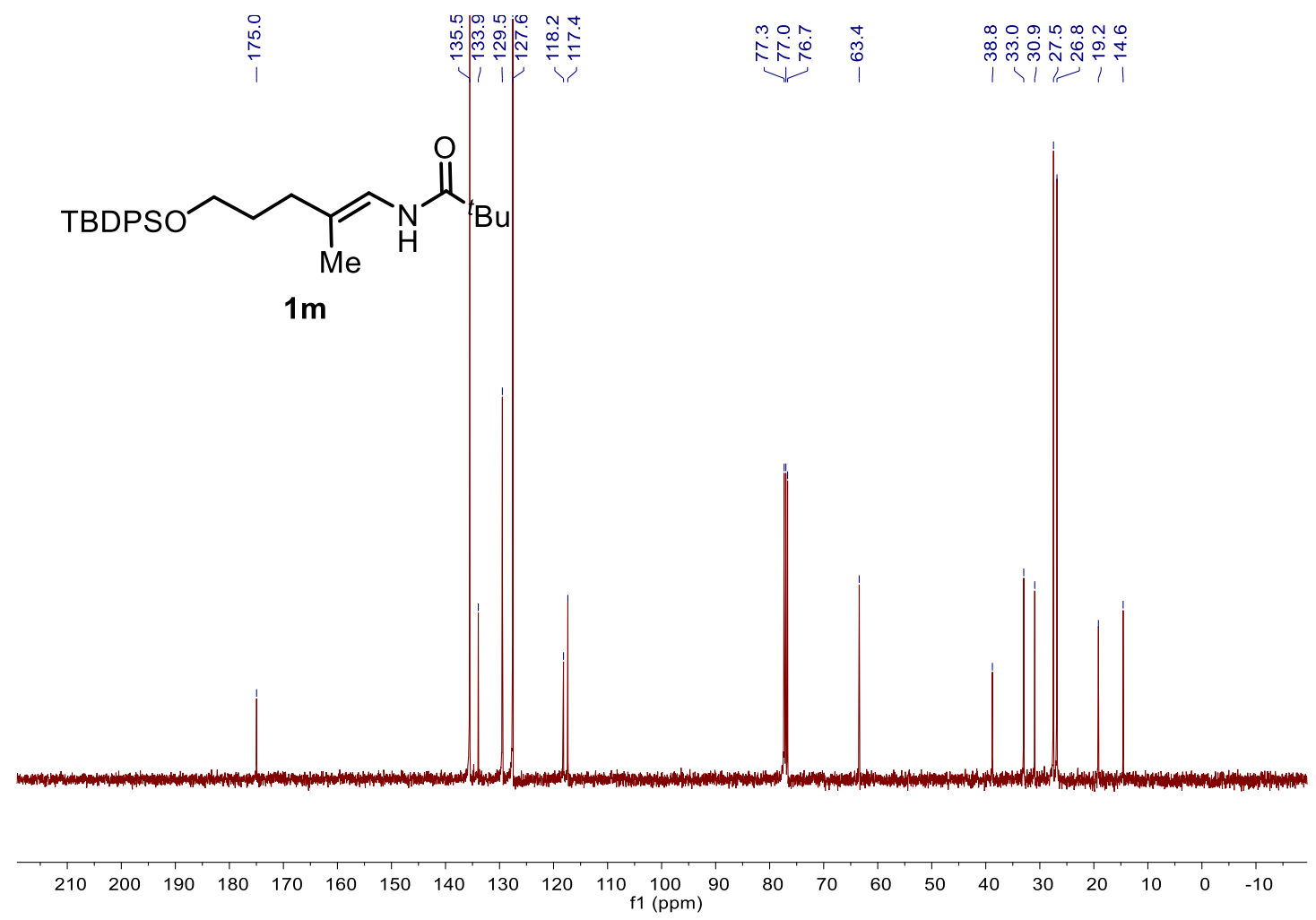

Figure S29. ${ }^{13} \mathbf{C}$ NMR $\left(101 \mathrm{MHz}, \mathrm{CDCl}_{3}\right)$ of $\mathbf{1 m}$ 


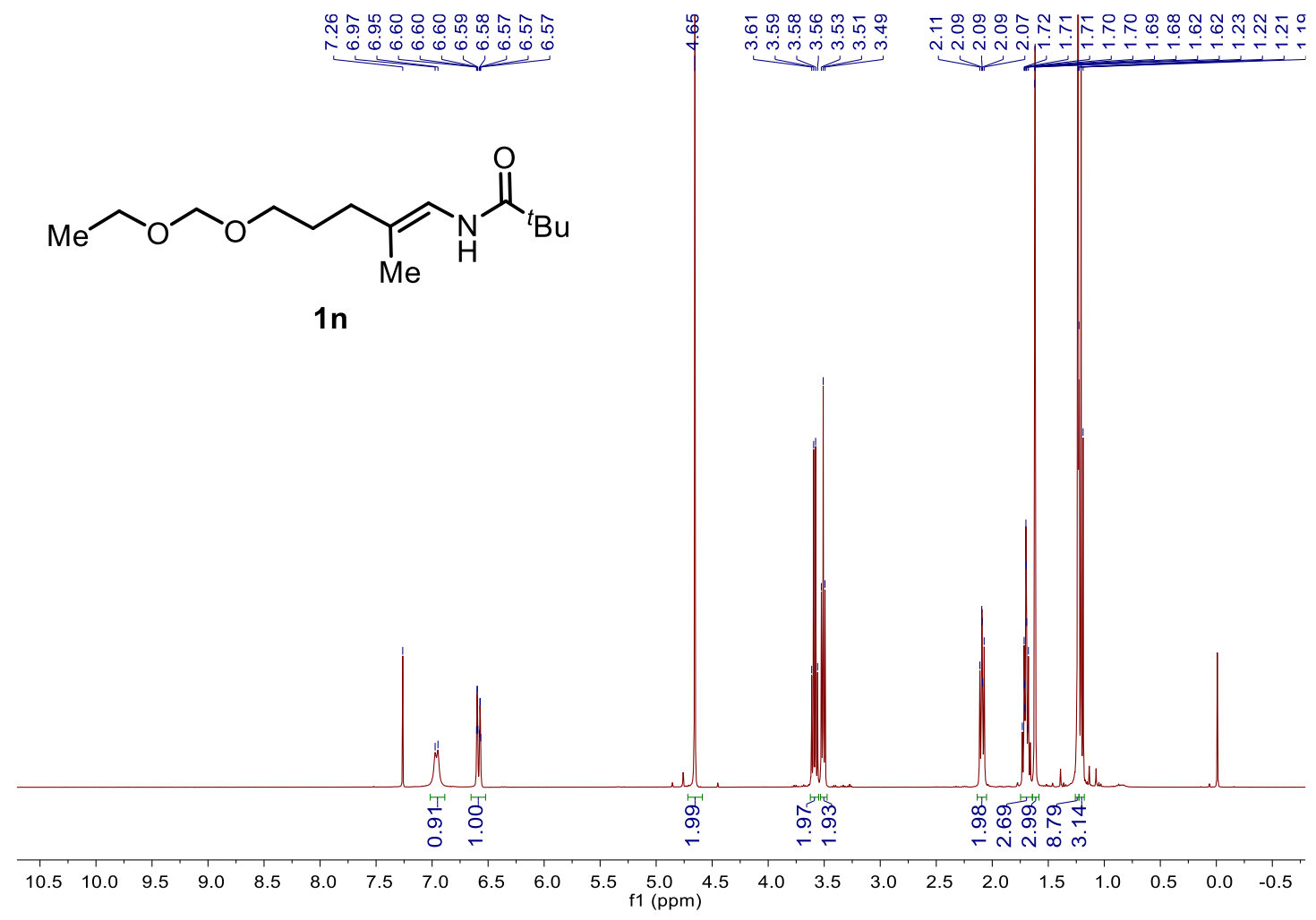

Figure S30. ${ }^{1} \mathbf{H}$ NMR $\left(400 \mathrm{MHz}, \mathrm{CDCl}_{3}\right)$ of $\mathbf{1 n}$

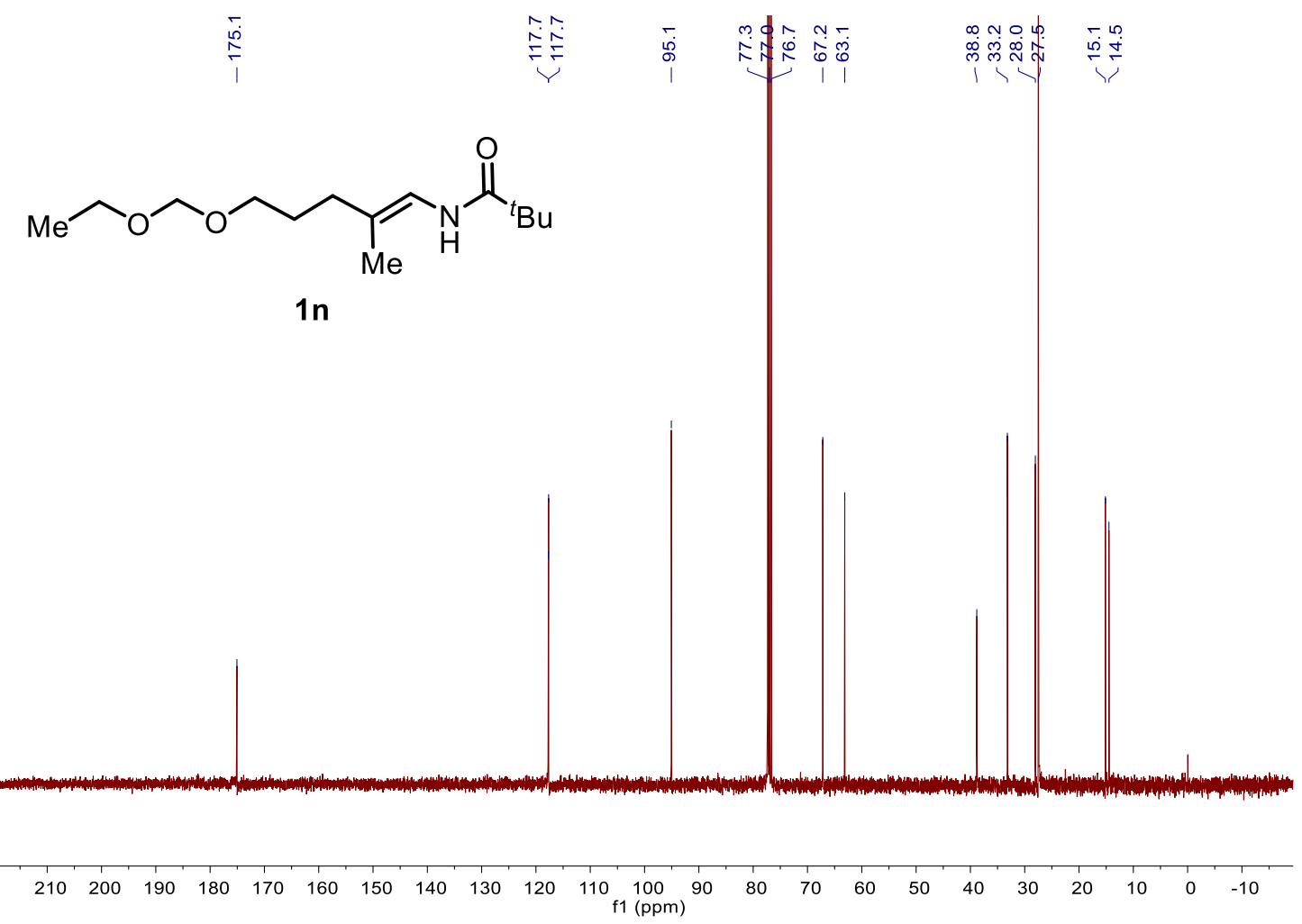

Figure S31. ${ }^{13} \mathbf{C}$ NMR $\left(101 \mathrm{MHz}, \mathrm{CDCl}_{3}\right)$ of $\mathbf{1 n}$ 


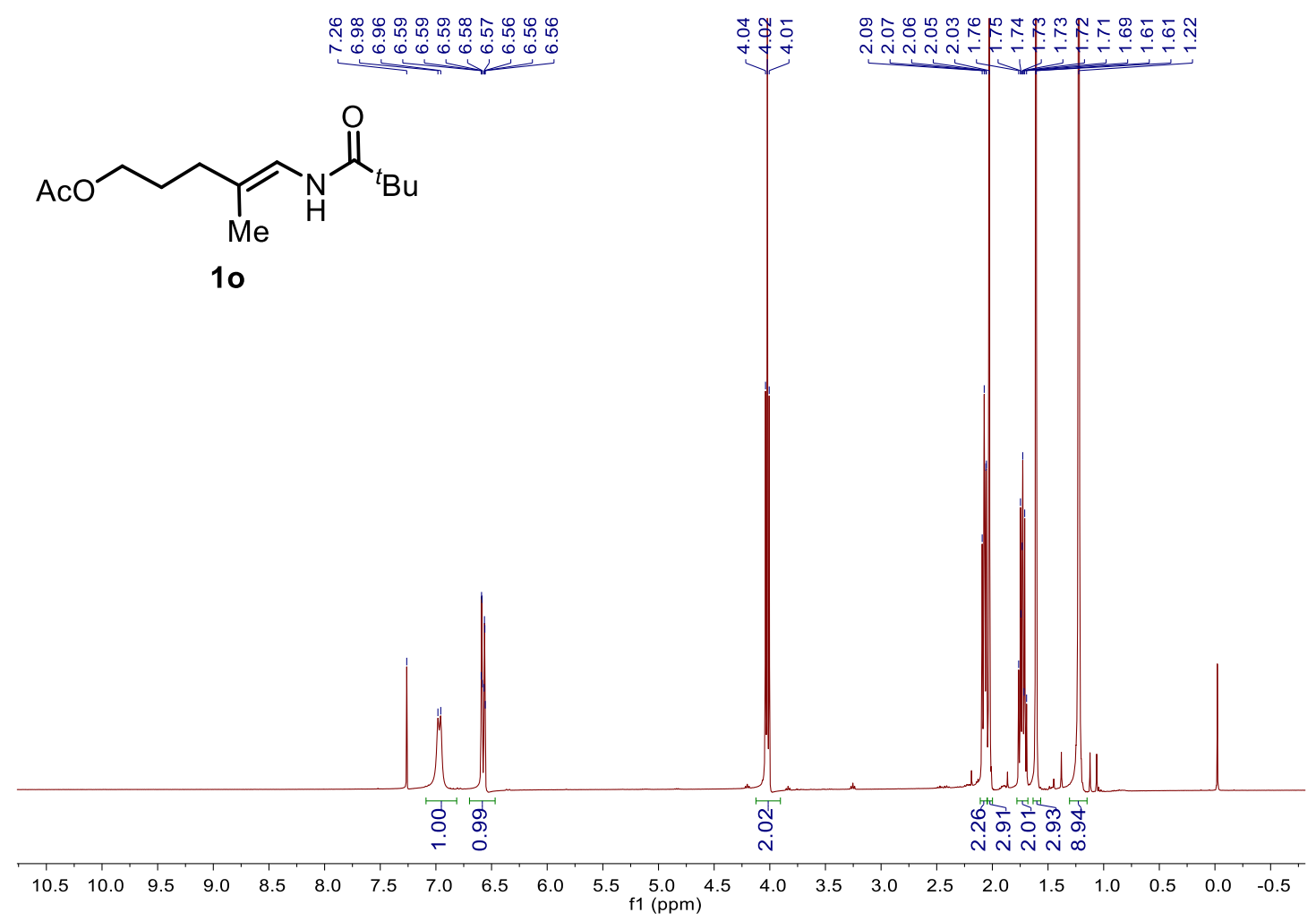

Figure S32. ${ }^{1} \mathbf{H}$ NMR $\left(400 \mathrm{MHz}, \mathrm{CDCl}_{3}\right)$ of $\mathbf{1 o}$

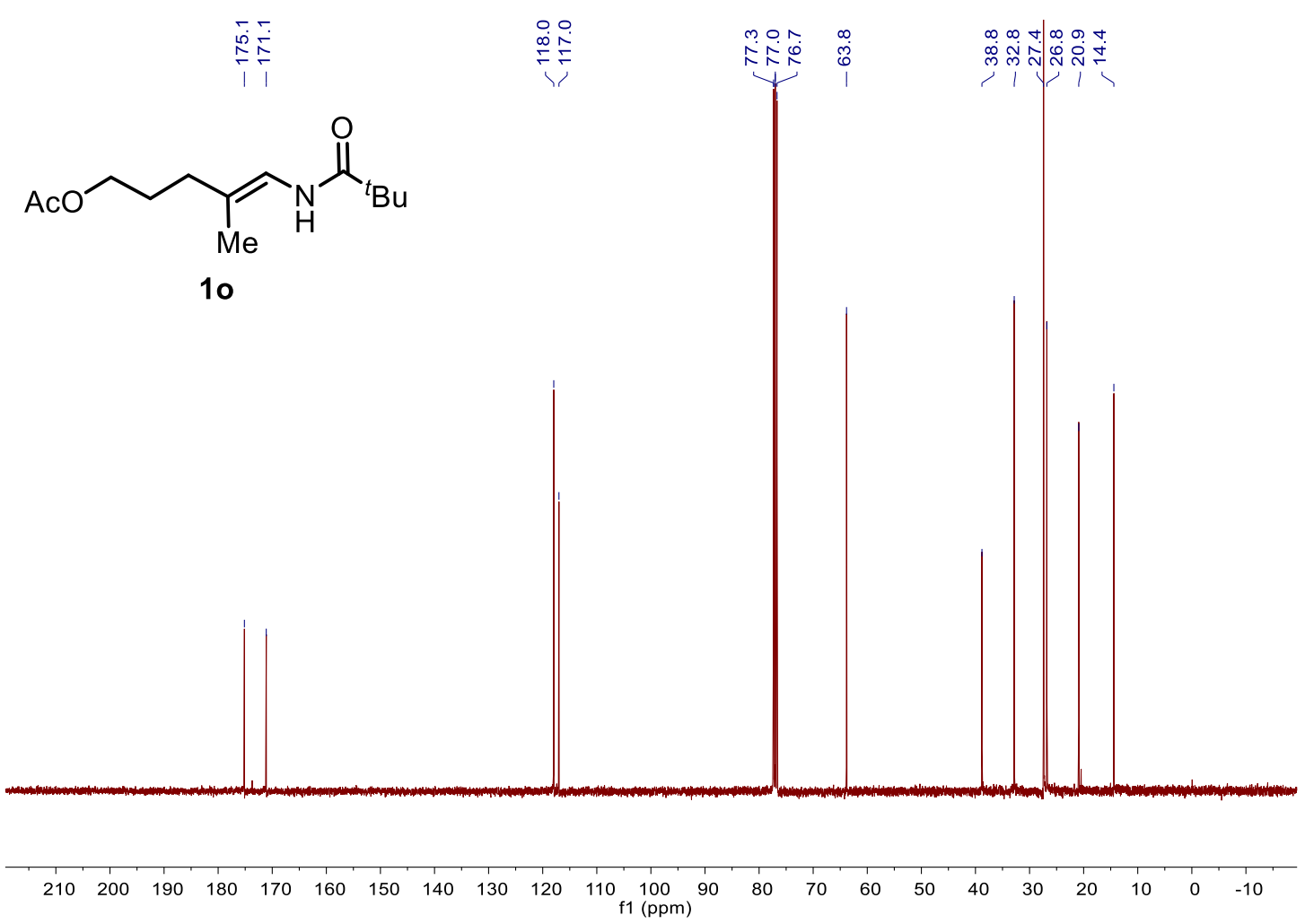

Figure S33. ${ }^{13} \mathbf{C}$ NMR $\left(101 \mathrm{MHz}, \mathrm{CDCl}_{3}\right)$ of $\mathbf{1 0}$ 


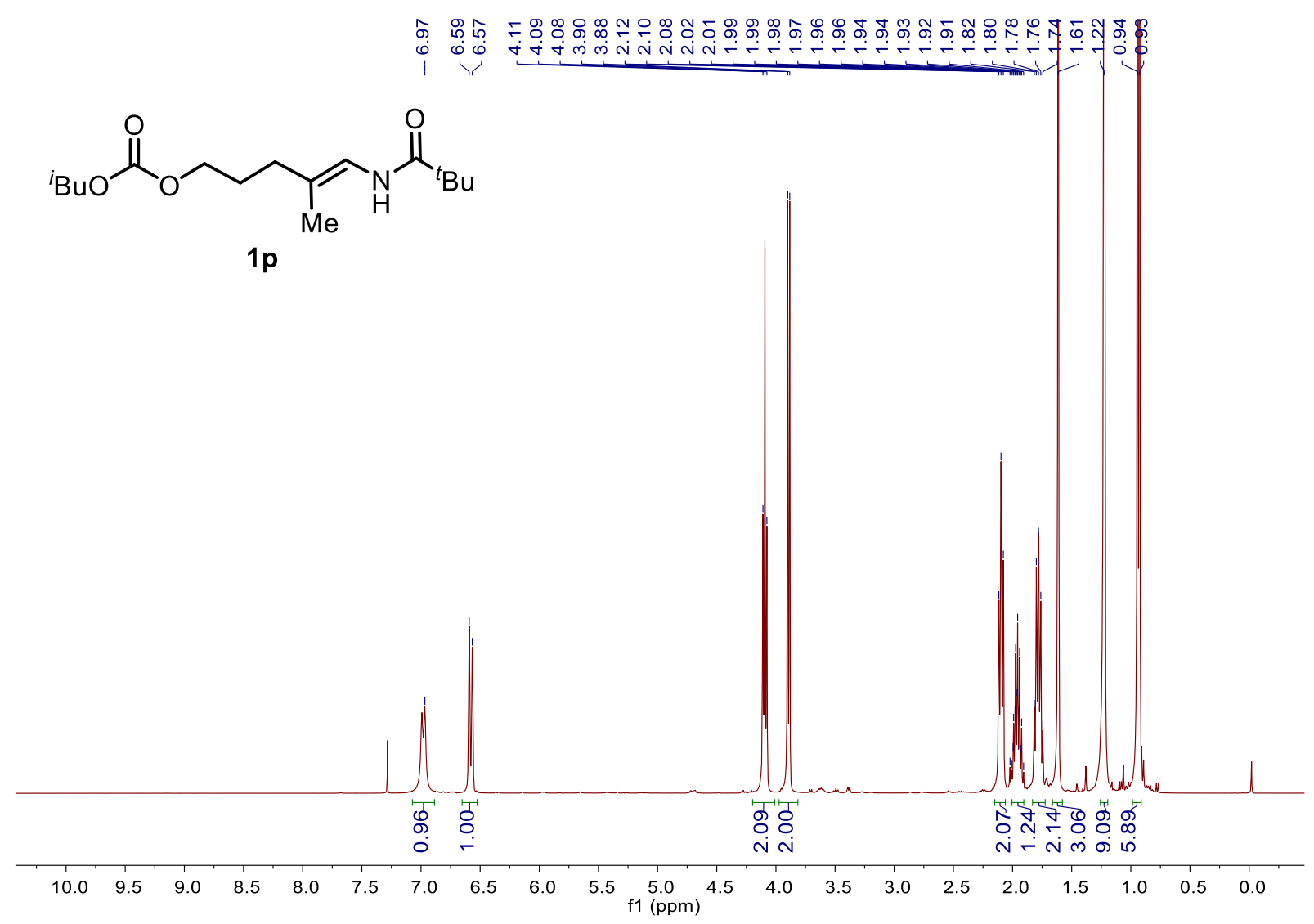

Figure S34. ${ }^{\mathbf{1}} \mathbf{H}$ NMR $\left(400 \mathrm{MHz}, \mathrm{CDCl}_{3}\right)$ of $\mathbf{1 p}$

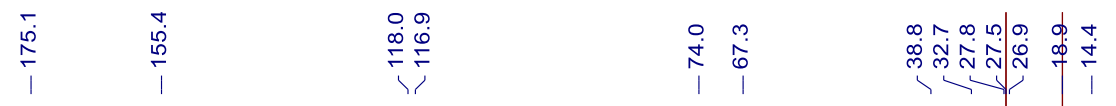<smiles>CCC(=O)OCCC/C(C)=C/NC(=O)OCCCC(C)(C)C</smiles>

$1 p$

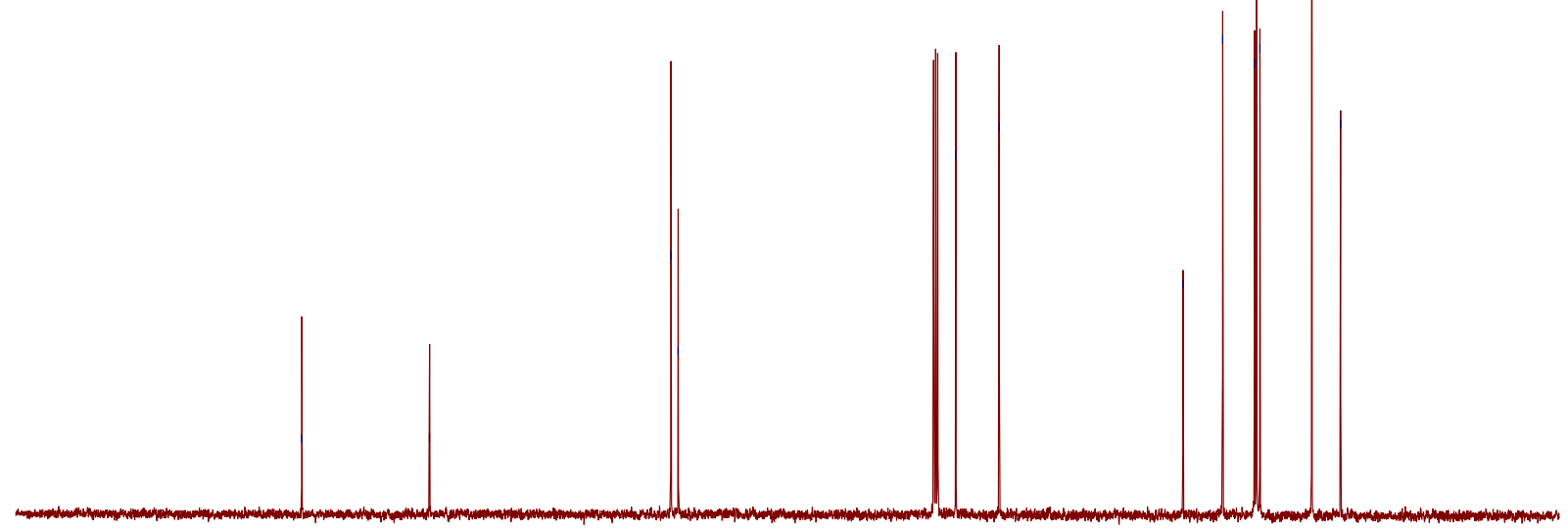

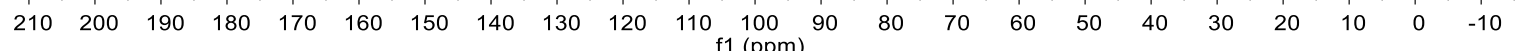

Figure S35. ${ }^{13} \mathbf{C}$ NMR $\left(101 \mathrm{MHz}, \mathrm{CDCl}_{3}\right)$ of $\mathbf{1 p}$ 


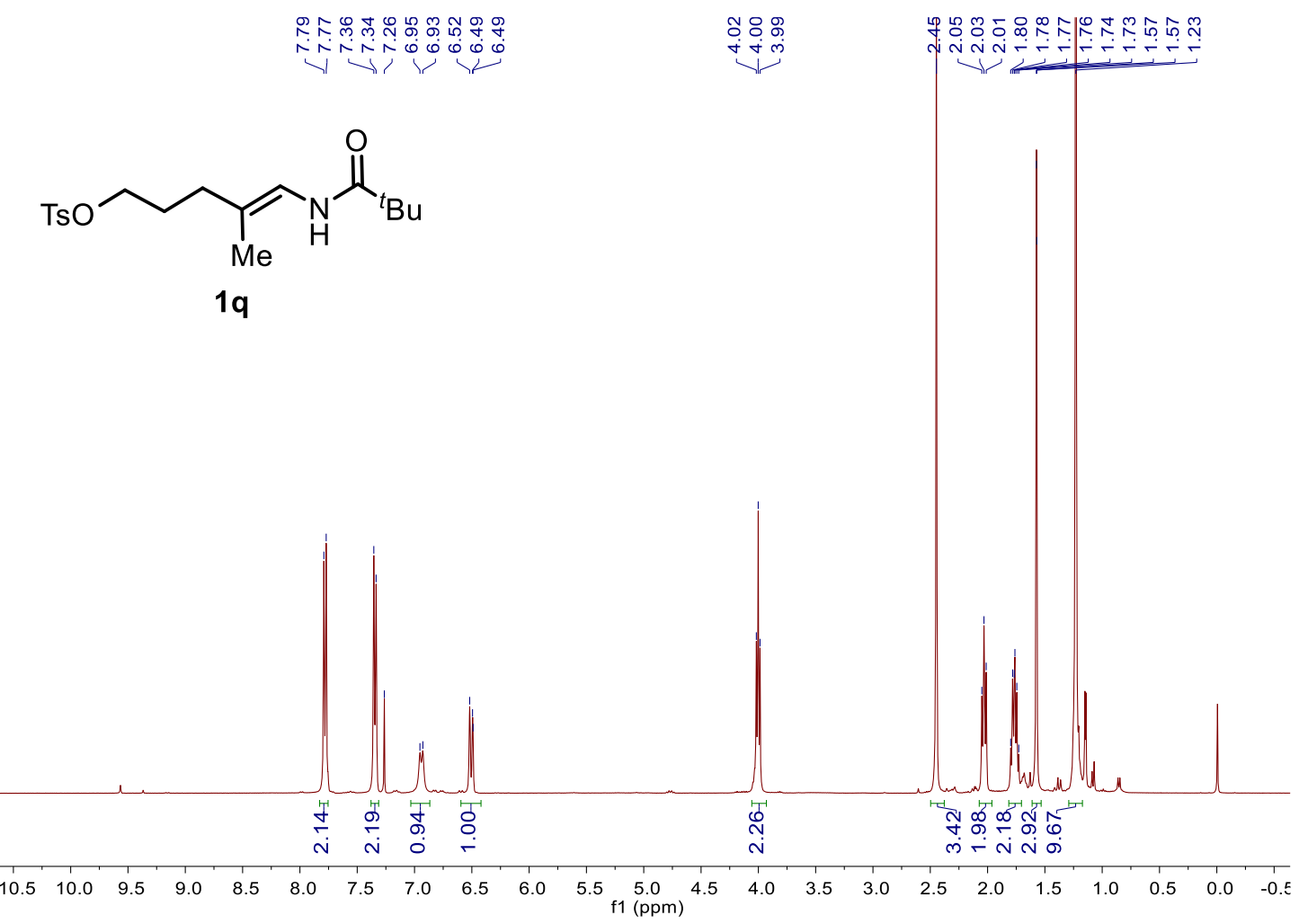

Figure S36. ${ }^{1} \mathbf{H}$ NMR $\left(400 \mathrm{MHz}, \mathrm{CDCl}_{3}\right)$ of $\mathbf{1 q}$

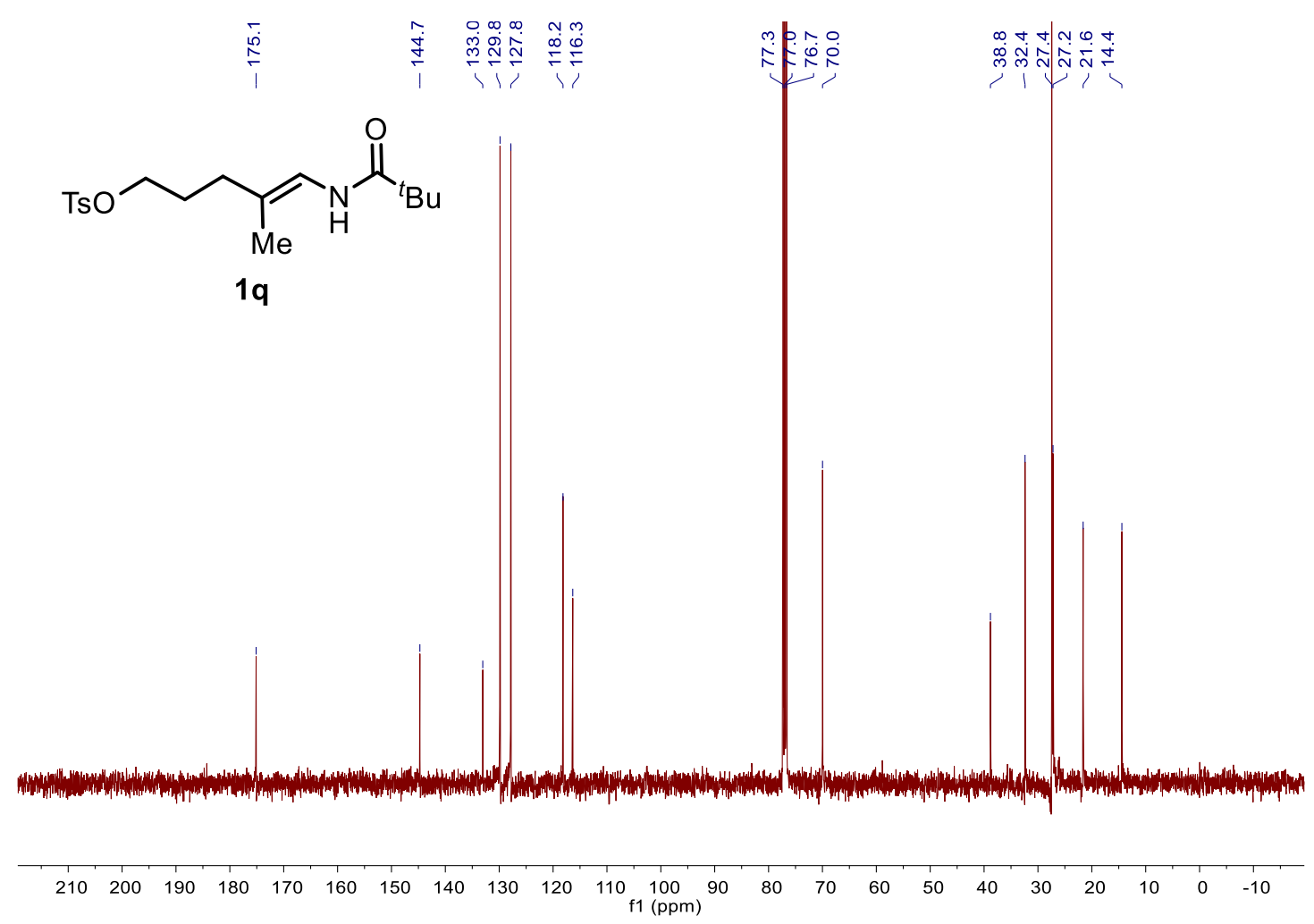

Figure S37. ${ }^{13} \mathbf{C}$ NMR $\left(101 \mathrm{MHz}, \mathrm{CDCl}_{3}\right)$ of $\mathbf{1 q}$ 


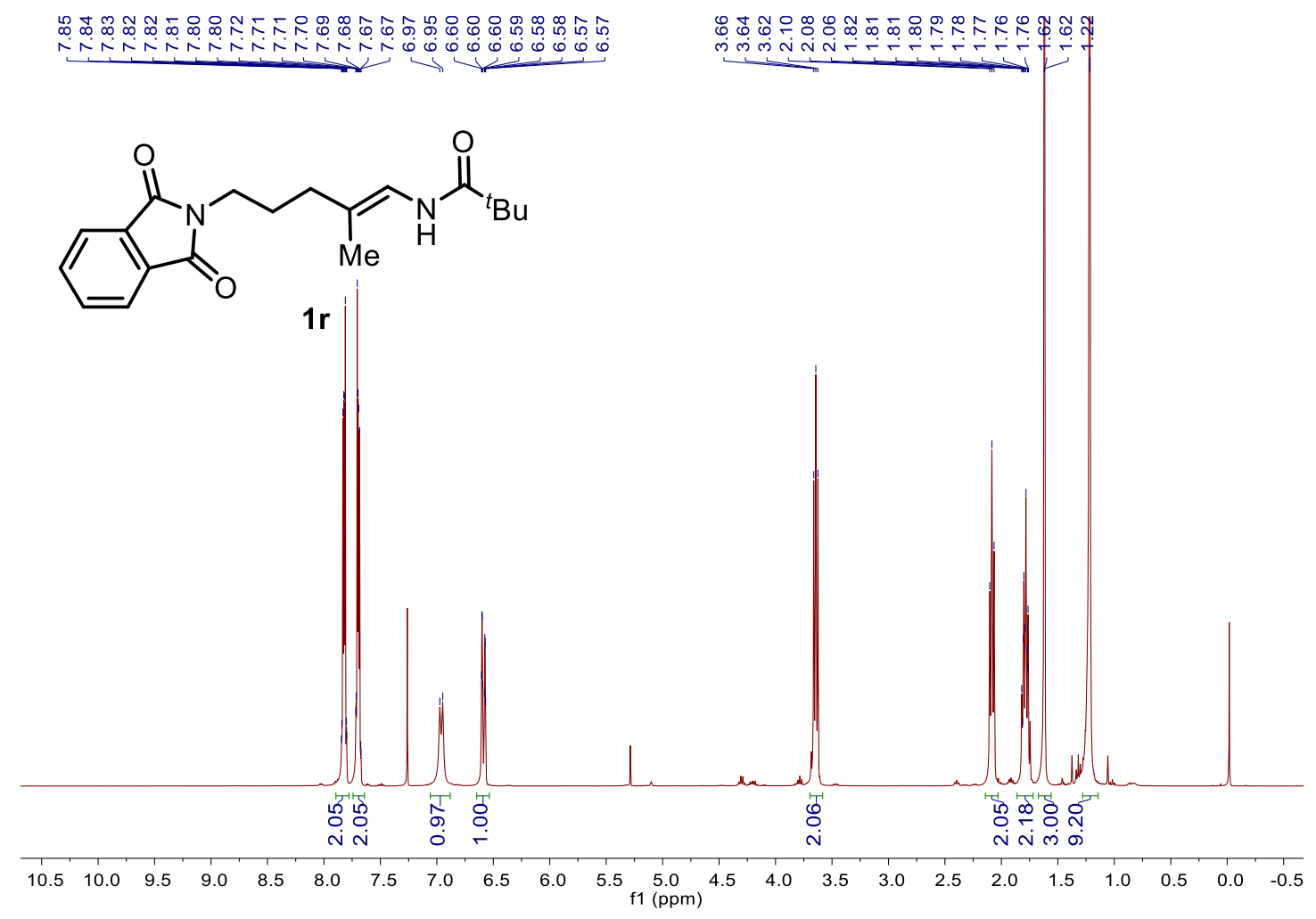

Figure S38. ${ }^{1} \mathbf{H}$ NMR $\left(400 \mathrm{MHz}, \mathrm{CDCl}_{3}\right)$ of $\mathbf{1 r}$

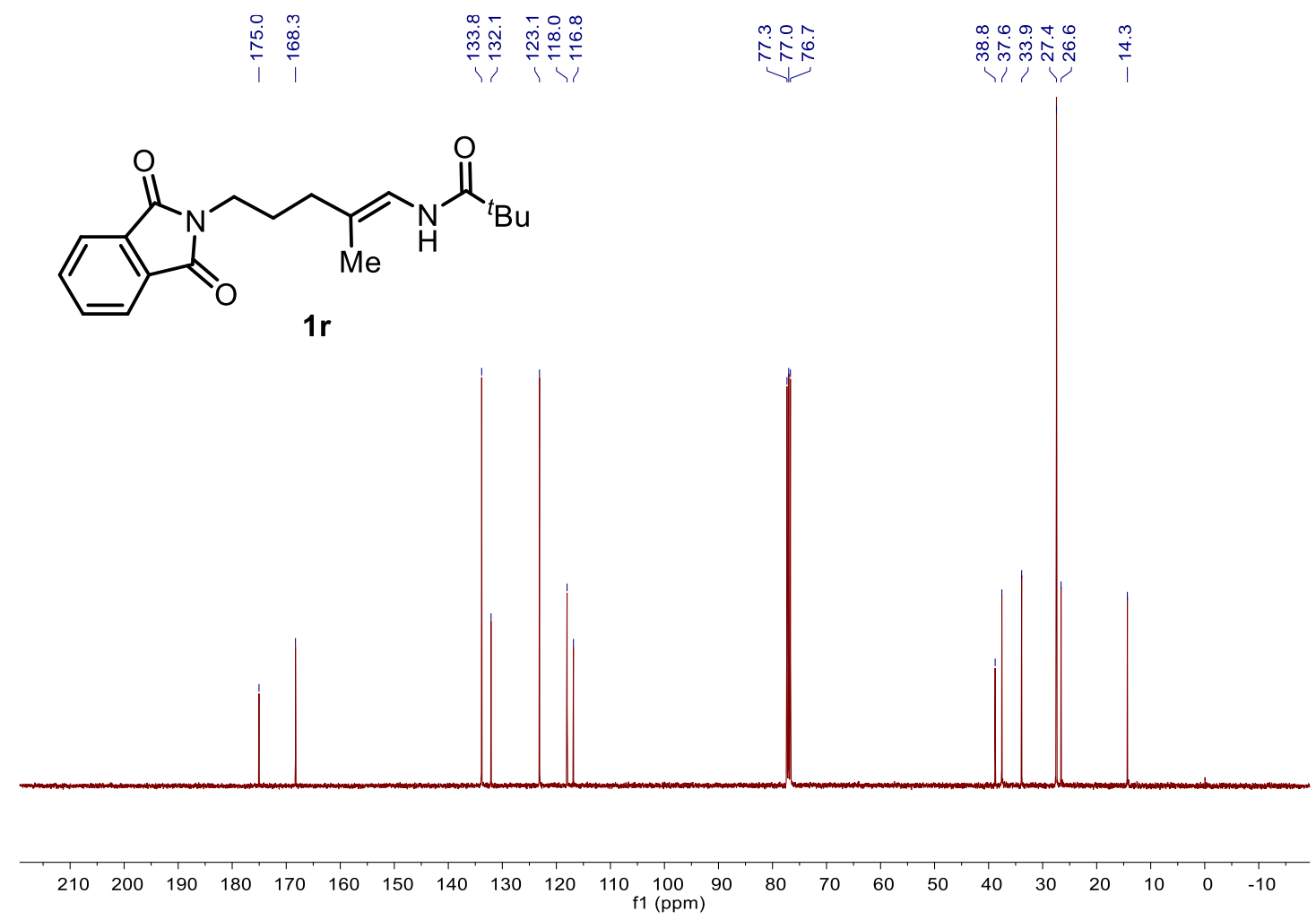

Figure S39. ${ }^{13} \mathbf{C}$ NMR $\left(101 \mathrm{MHz}, \mathrm{CDCl}_{3}\right)$ of $\mathbf{1 r}$ 
<smiles>CC(C)(C)CCc1ccc2ccccc2c1</smiles>

$1 \mathrm{~s}$

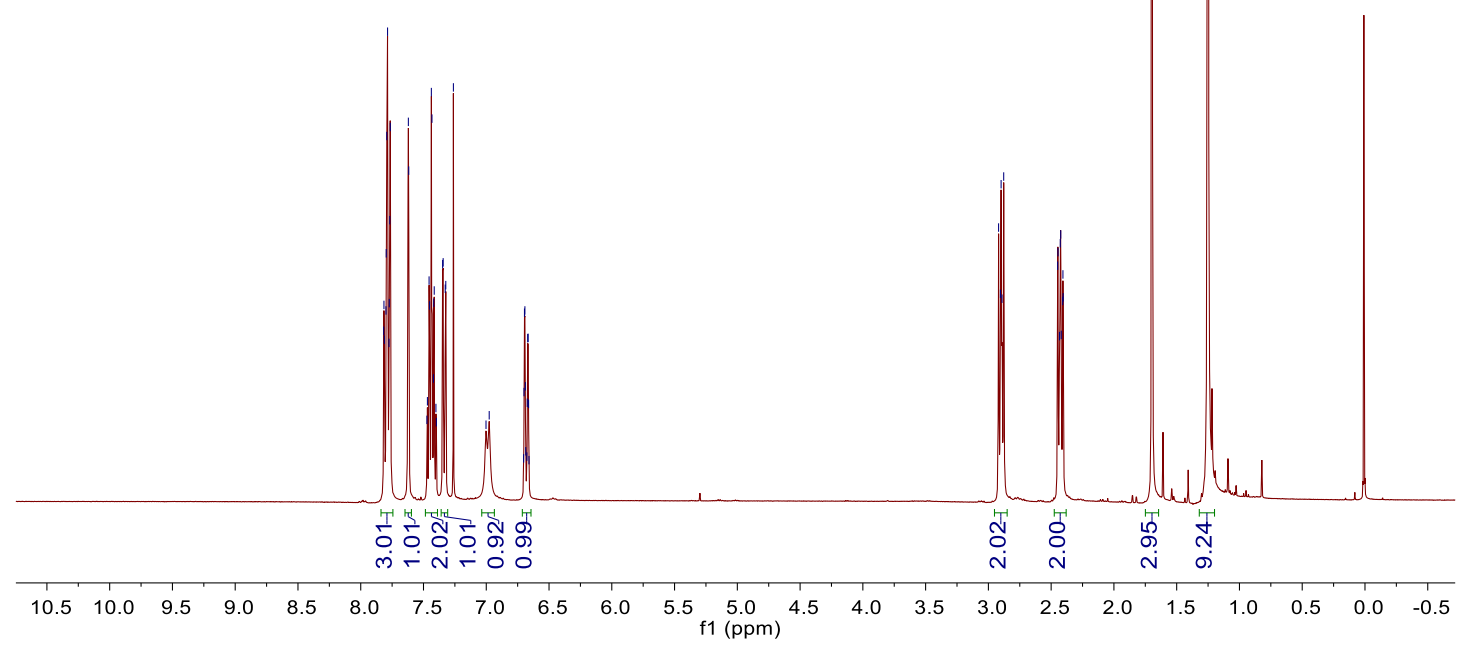

Figure S40. ${ }^{1} \mathbf{H}$ NMR $\left(400 \mathrm{MHz}, \mathrm{CDCl}_{3}\right)$ of $\mathbf{1 s}$

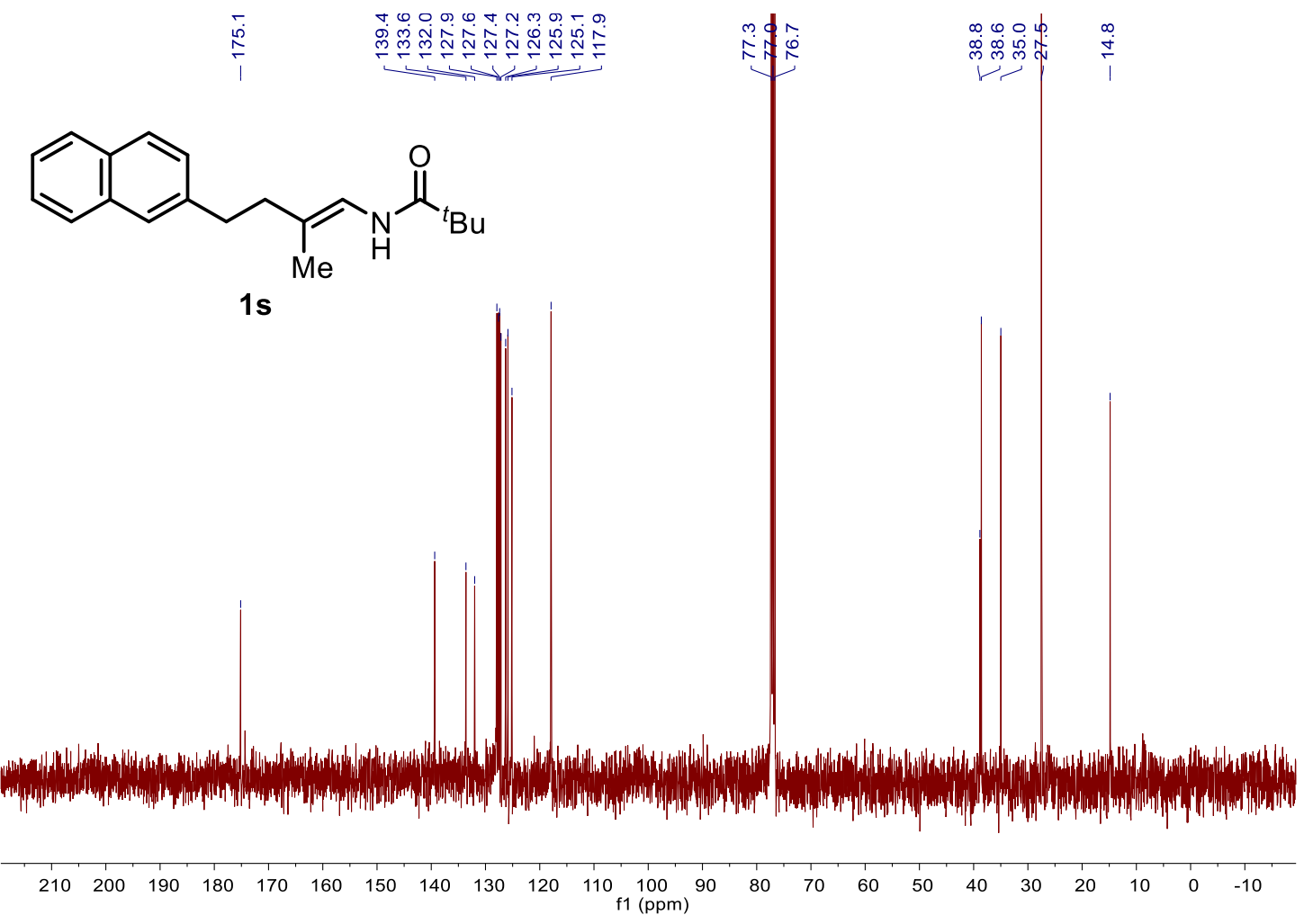

Figure $\mathbf{S 4 1} .{ }^{13} \mathbf{C ~ N M R}\left(101 \mathrm{MHz}, \mathrm{CDCl}_{3}\right)$ of $\mathbf{1 s}$ 


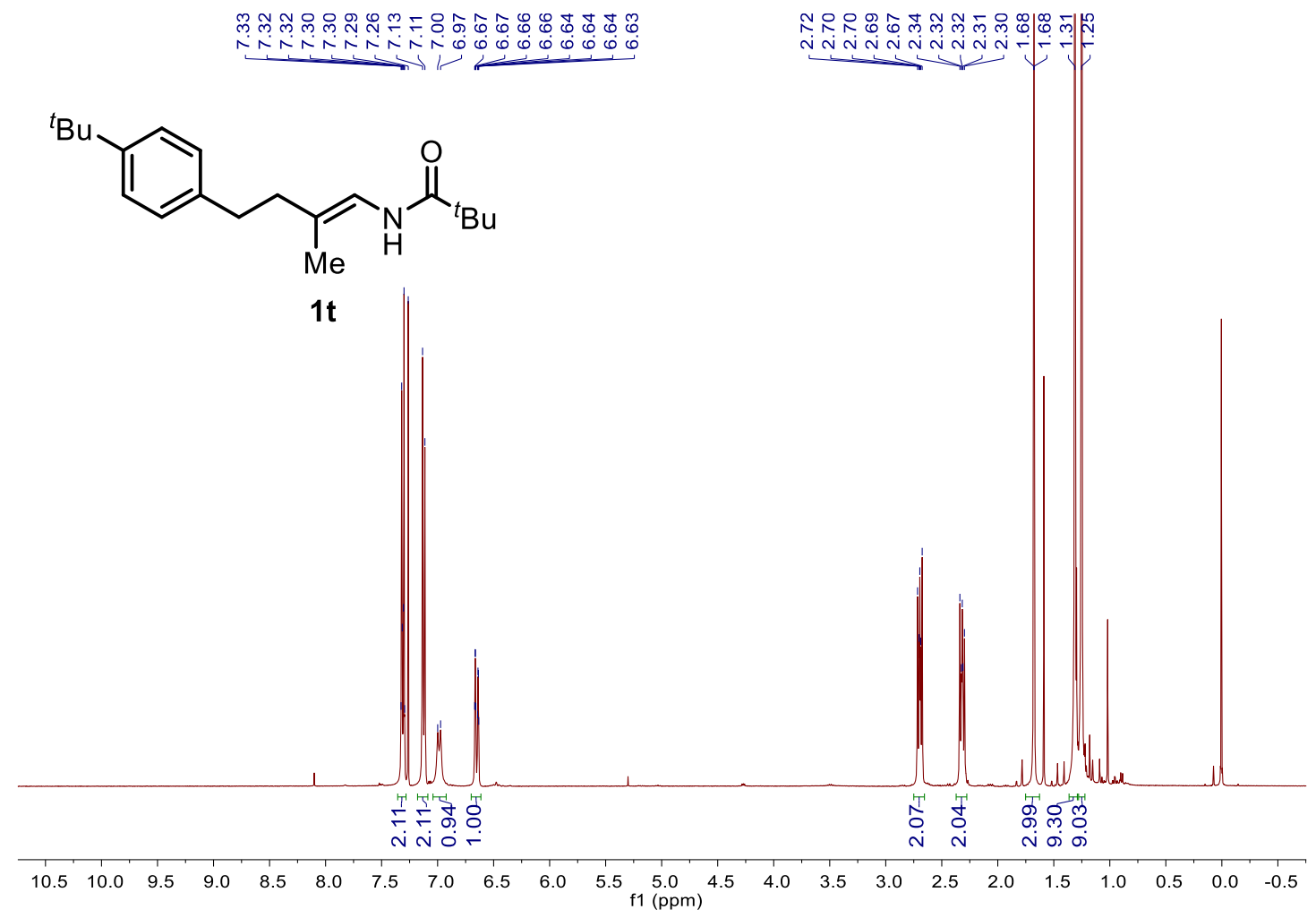

Figure S42. ${ }^{1} \mathbf{H}$ NMR $\left(400 \mathrm{MHz}, \mathrm{CDCl}_{3}\right)$ of $\mathbf{1 t}$

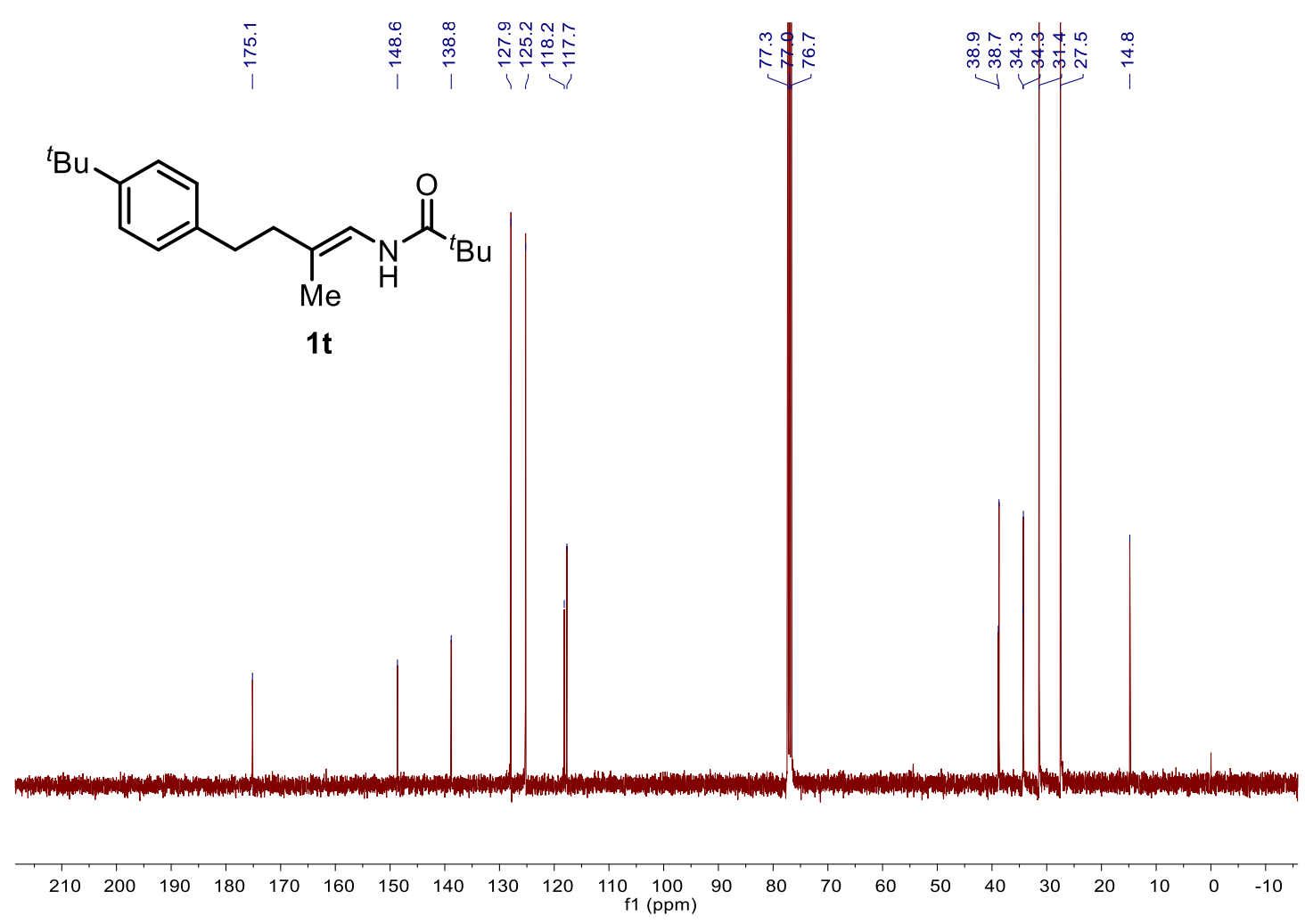

Figure S43. ${ }^{13} \mathbf{C}$ NMR $\left(101 \mathrm{MHz}, \mathrm{CDCl}_{3}\right)$ of $\mathbf{1 t}$ 


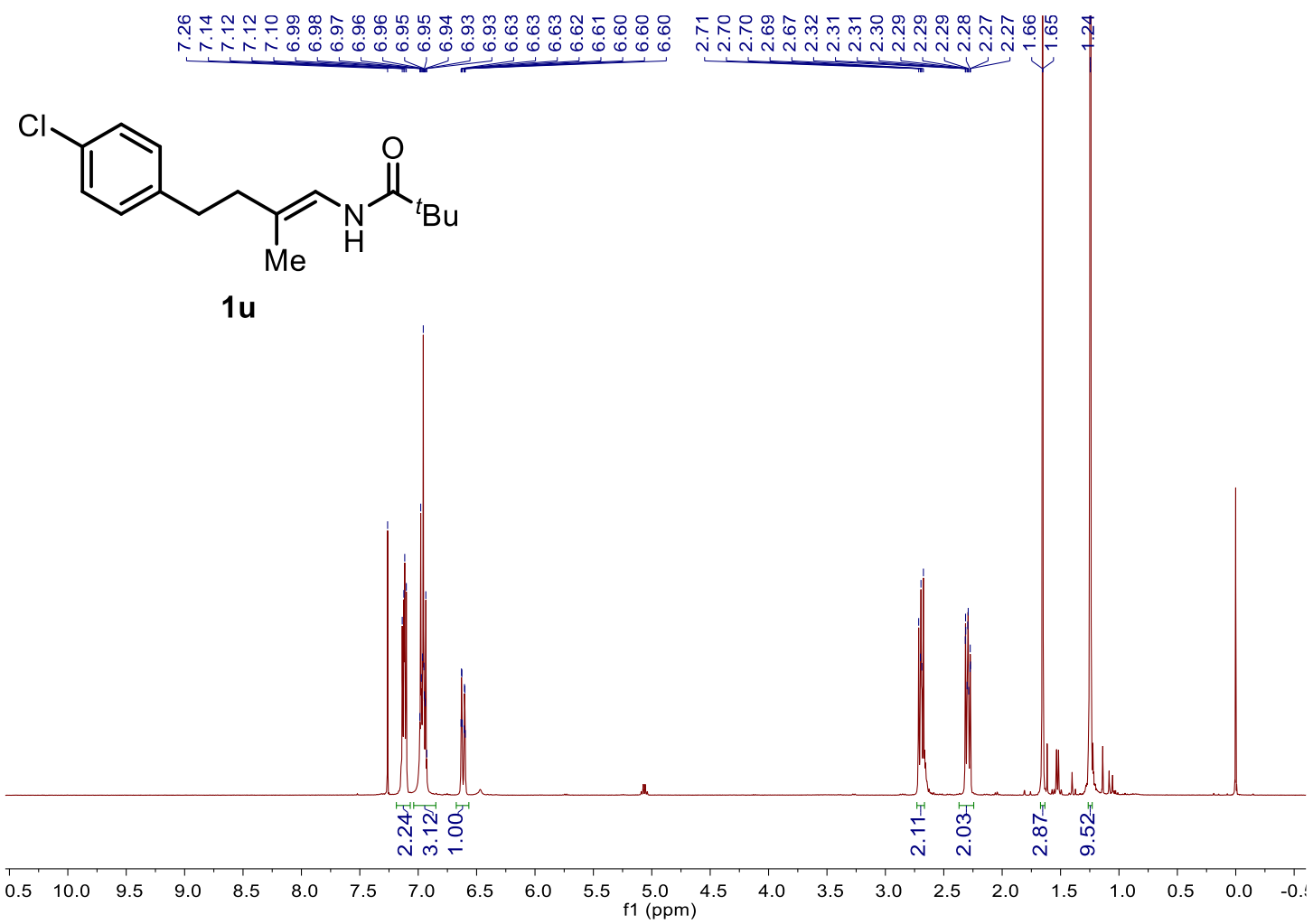

Figure S44. ${ }^{1} \mathbf{H}$ NMR $\left(400 \mathrm{MHz}, \mathrm{CDCl}_{3}\right)$ of $\mathbf{1 u}$<smiles>CC(C)=CNC(=O)Br</smiles>

$1 \mathrm{u}$<smiles>CC(C)(C)CCc1ccc(Cl)cc1</smiles>

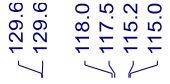

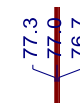

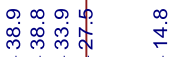

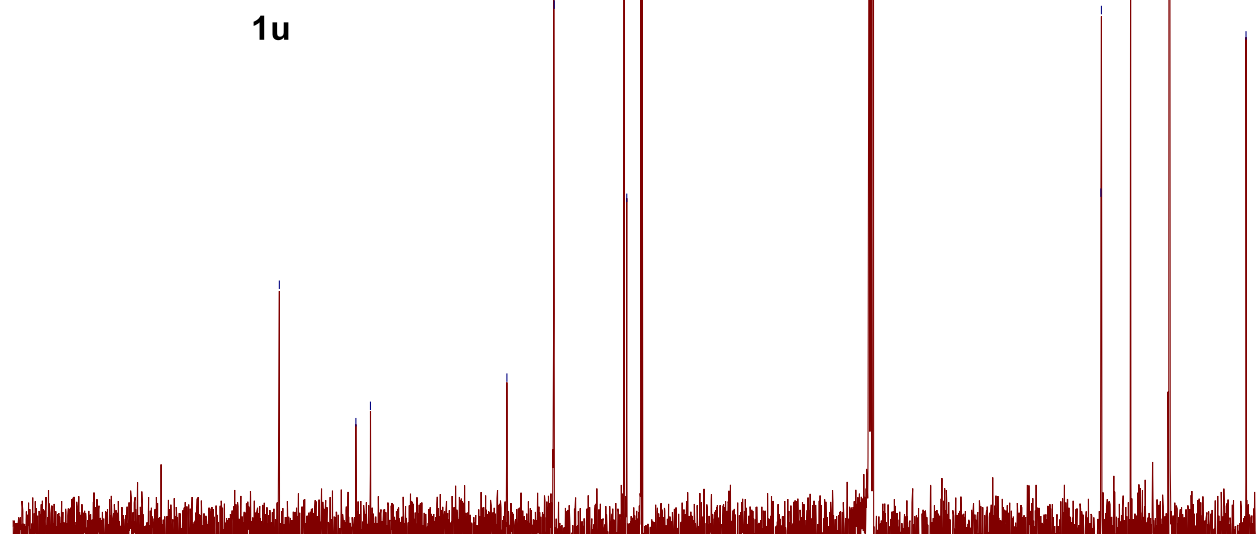

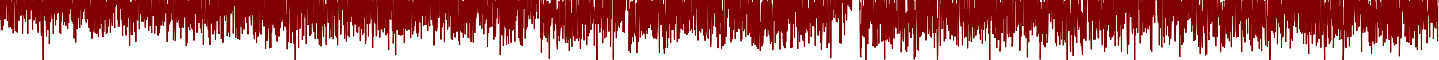

$\begin{array}{lllllllllllllllllllllll}210 & 200 & 190 & 180 & 170 & 160 & 150 & 140 & 130 & 120 & 110 & 100 & 90 & 80 & 70 & 60 & 50 & 40 & 30 & 20 & 10 & 0 & -10\end{array}$

Figure S45. ${ }^{13} \mathbf{C}$ NMR $\left(101 \mathrm{MHz}, \mathrm{CDCl}_{3}\right)$ of $\mathbf{1 u}$ 
$\mathrm{BnO}$<smiles>CC(=CNC(=O)Cc1ccccc1)CCc1ccc(CCC(C)(C)C)cc1</smiles>

1v

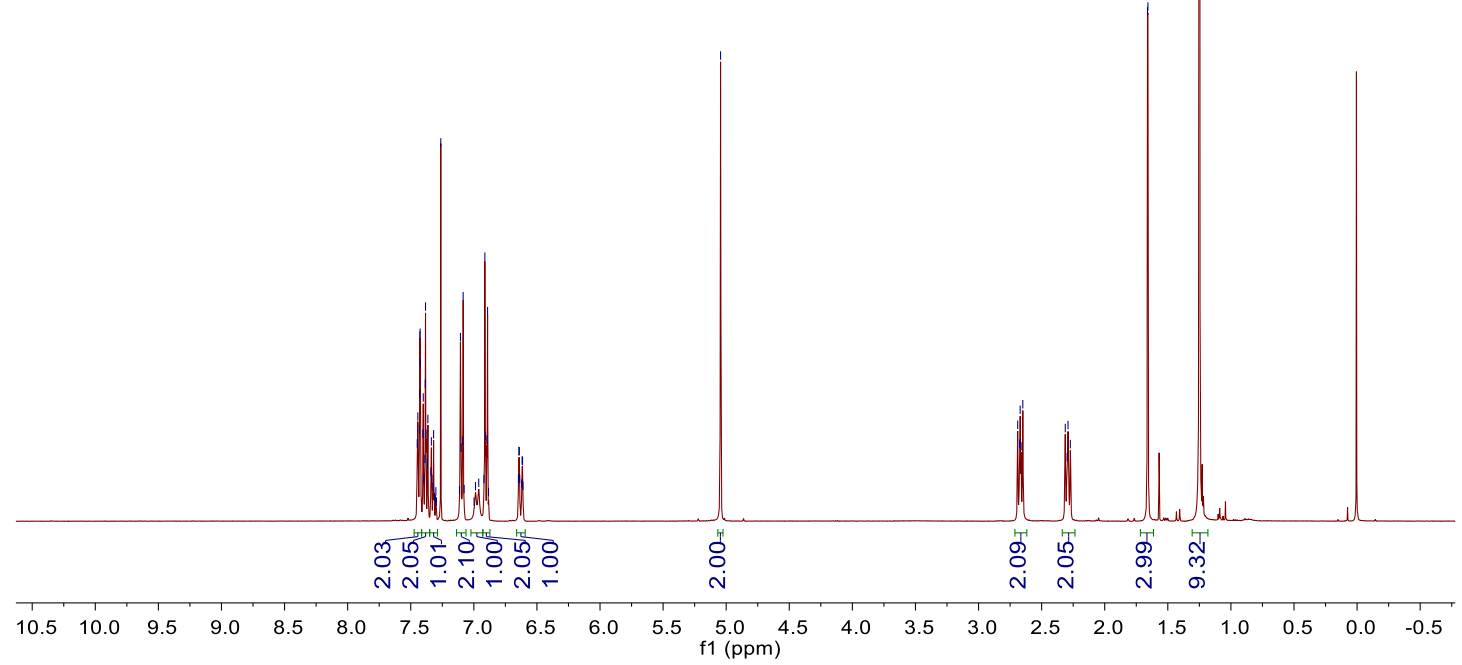

Figure S46. ${ }^{1} \mathbf{H}$ NMR $\left(400 \mathrm{MHz}, \mathrm{CDCl}_{3}\right)$ of $\mathbf{1 v}$

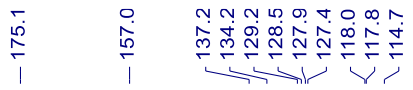

$\mathrm{BnO}$<smiles>CC(=CNC(=O)CCc1ccc(I)cc1)CCc1ccccc1</smiles>

1v

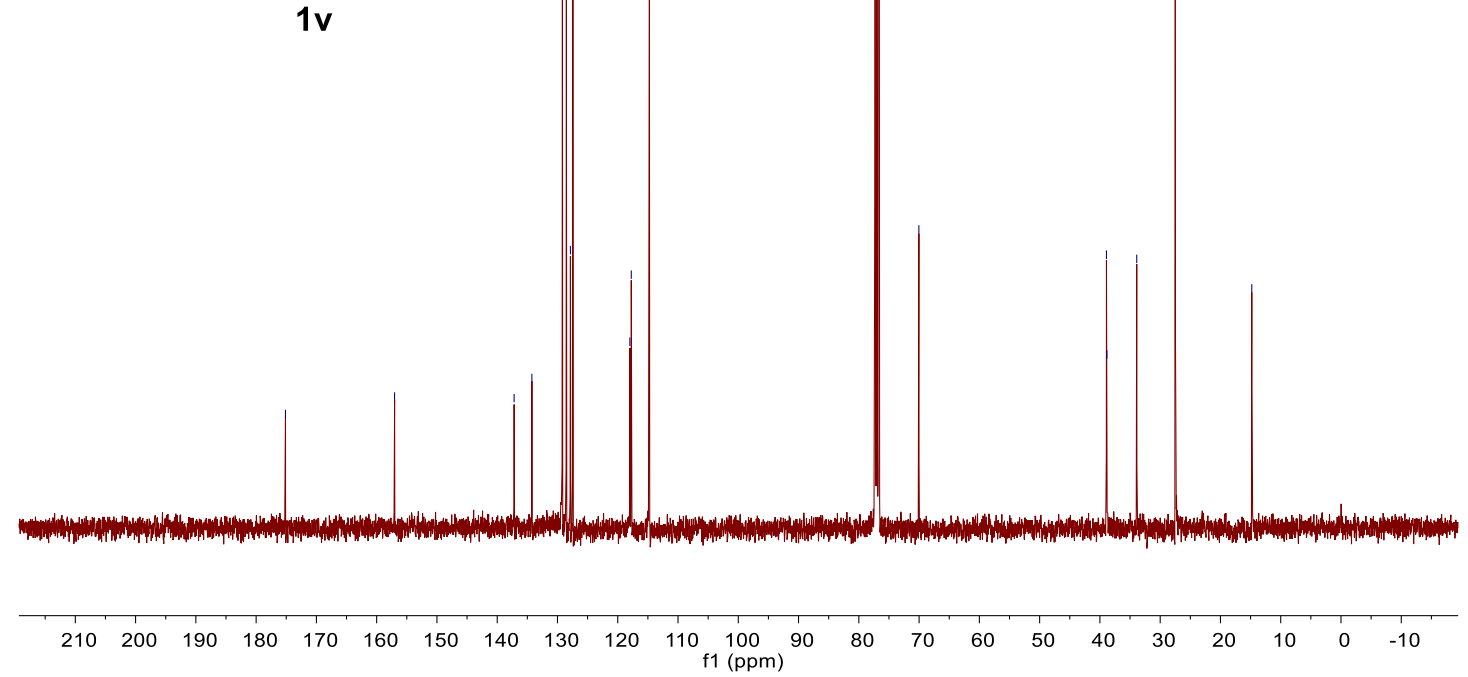

Figure S47. ${ }^{13} \mathbf{C}$ NMR $\left(101 \mathrm{MHz}, \mathrm{CDCl}_{3}\right)$ of $\mathbf{1 v}$ 


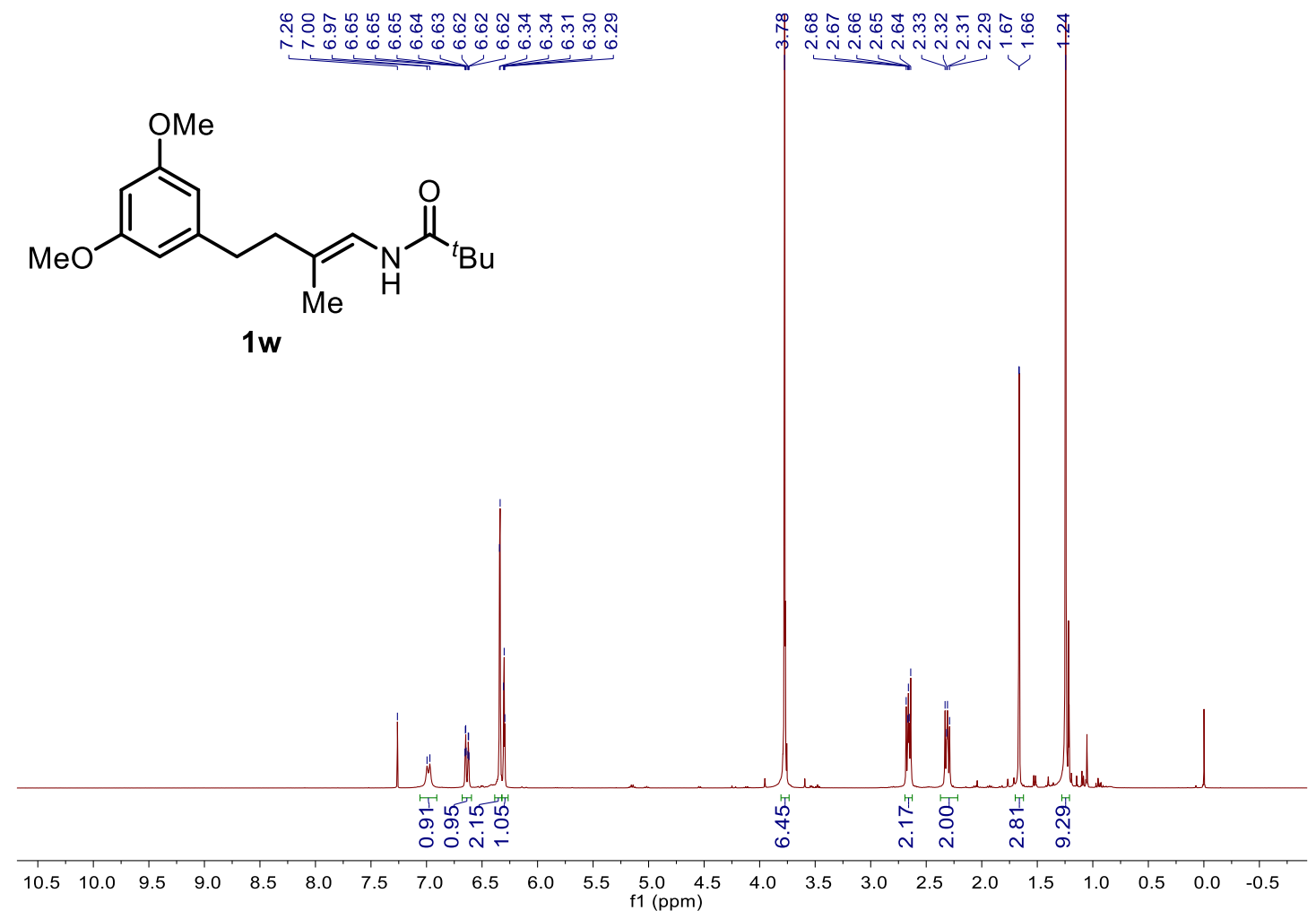

Figure S48. ${ }^{1} \mathbf{H}$ NMR $\left(400 \mathrm{MHz}, \mathrm{CDCl}_{3}\right)$ of $\mathbf{1 w}$

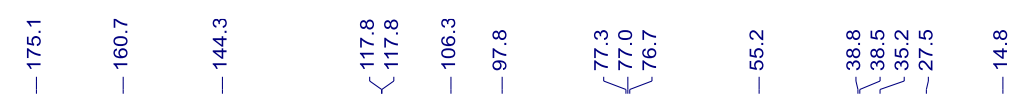<smiles>COc1cc(CC/C(C)=C/NC(=O)C(C)(C)C)cc(OC)c1</smiles>

$1 w$

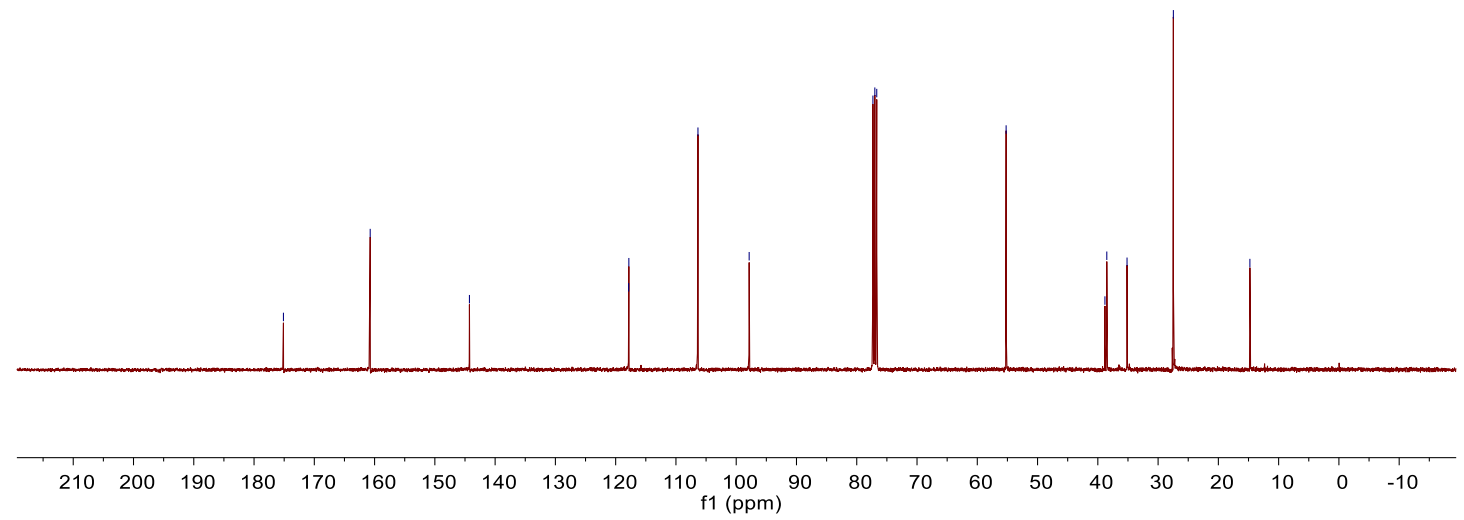

Figure S49. ${ }^{13} \mathbf{C}$ NMR $\left(101 \mathrm{MHz}, \mathrm{CDCl}_{3}\right)$ of $\mathbf{1 w}$ 


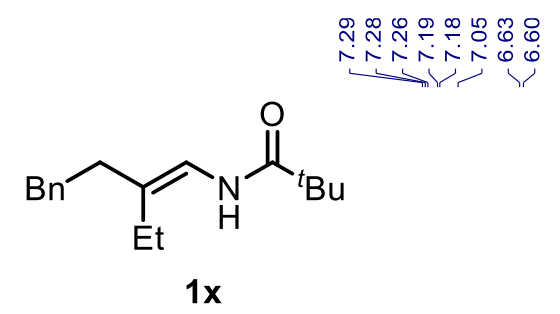

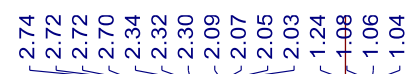

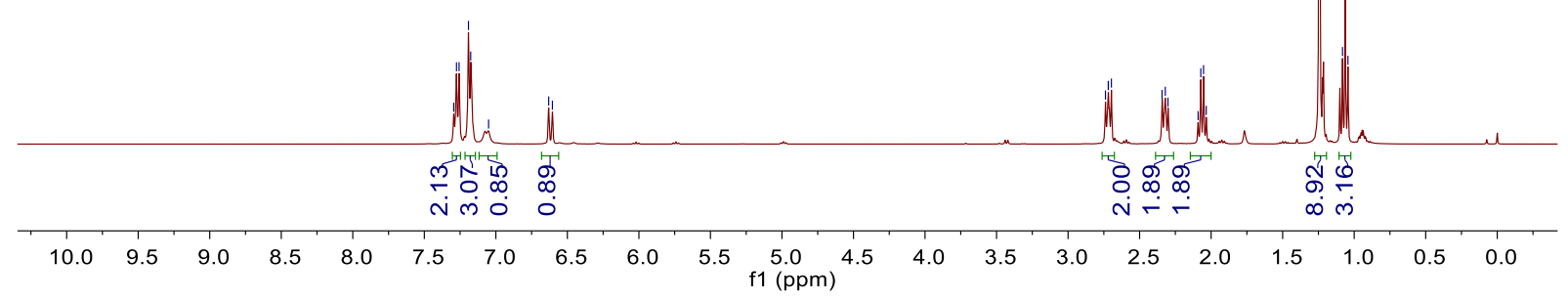

Figure S50. ${ }^{1} \mathbf{H}$ NMR $\left(400 \mathrm{MHz}, \mathrm{CDCl}_{3}\right)$ of $\mathbf{1 x}$

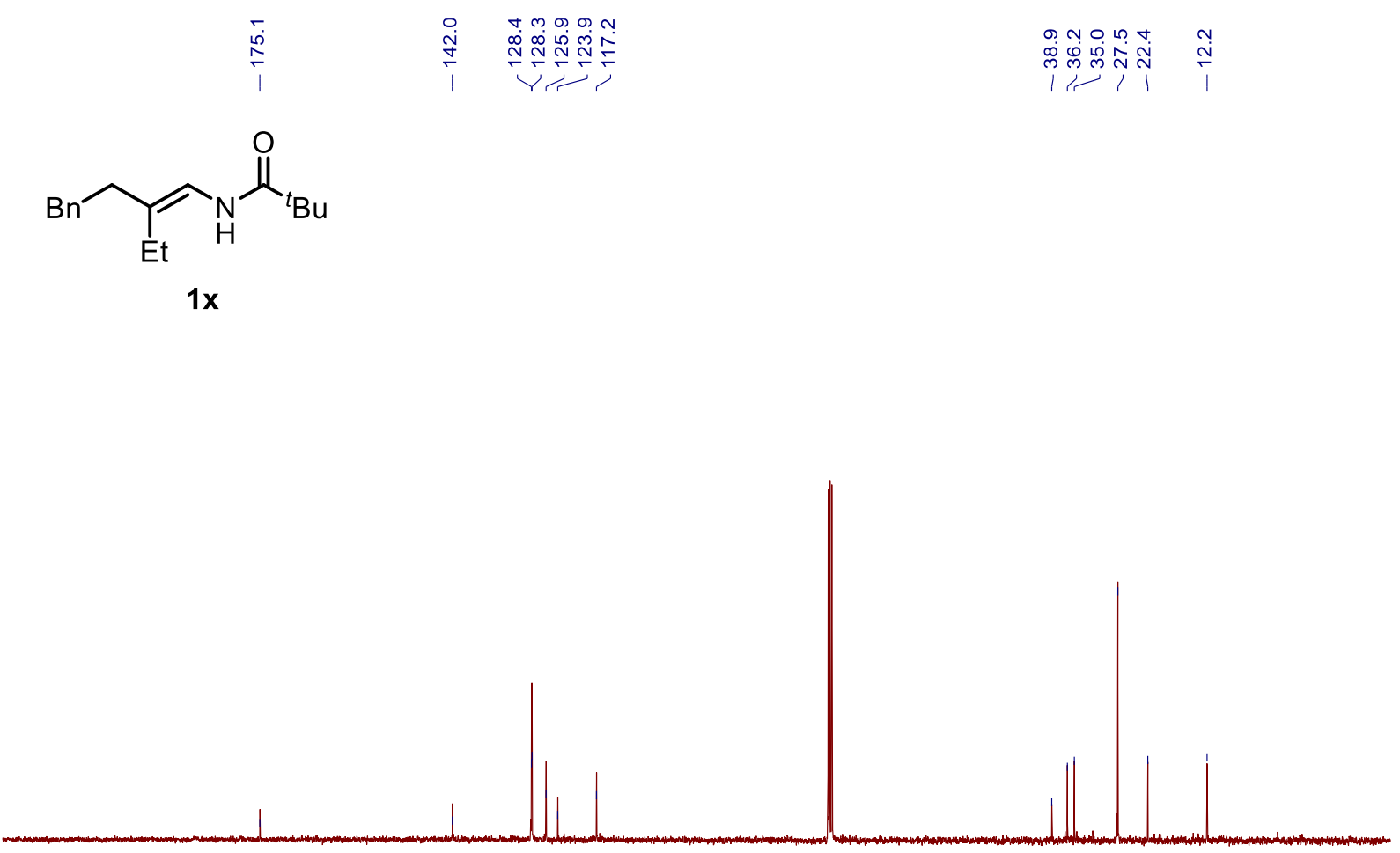

$\begin{array}{lllllllllllllllllllllllllllll}210 & 200 & 190 & 180 & 170 & 160 & 150 & 140 & 130 & 120 & 110 & 100 & 90 & 80 & 70 & 60 & 50 & 40 & 30 & 20 & 10 & 0 & -10\end{array}$

Figure S51. ${ }^{13} \mathbf{C}$ NMR $\left(101 \mathrm{MHz}, \mathrm{CDCl}_{3}\right)$ of $\mathbf{1 x}$ 
<smiles>[Y20]CC(C)=CNC(=O)Cc1ccccc1</smiles>

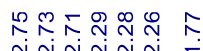

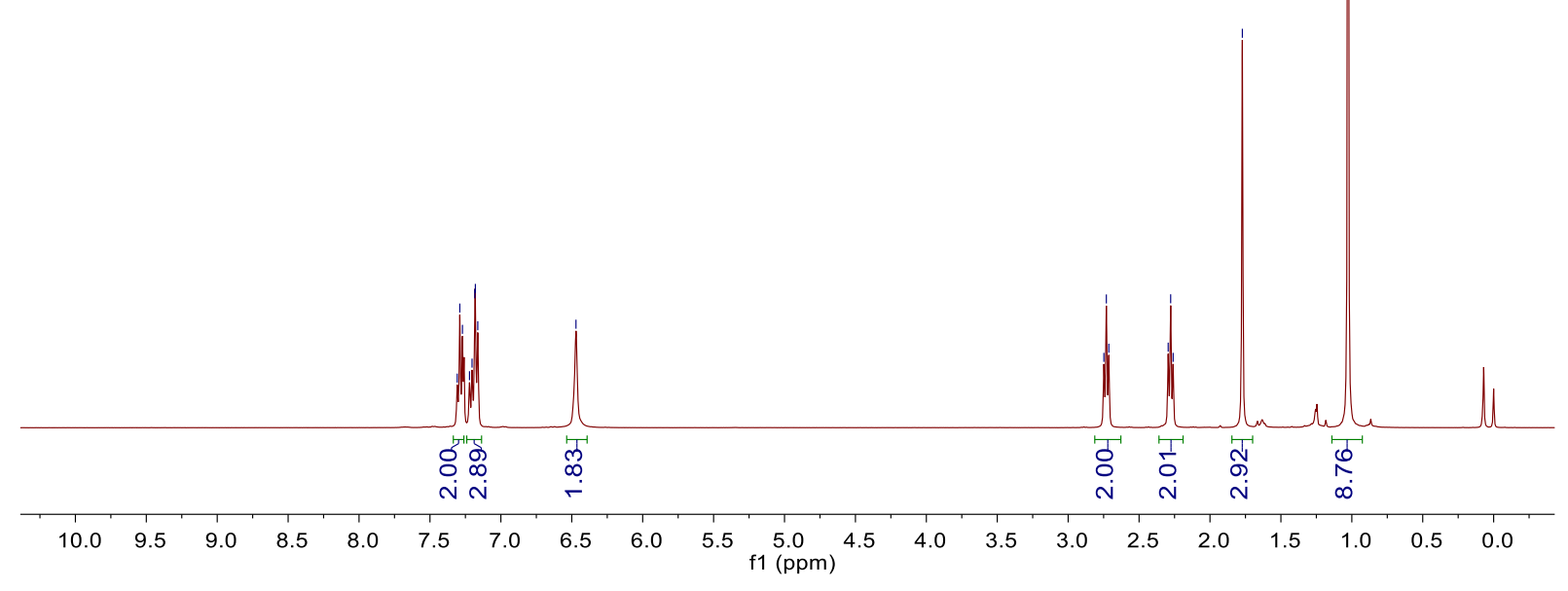

Figure S52. ${ }^{1} \mathbf{H}$ NMR $\left(400 \mathrm{MHz}, \mathrm{CDCl}_{3}\right)$ of $\mathbf{1 a - Z}$

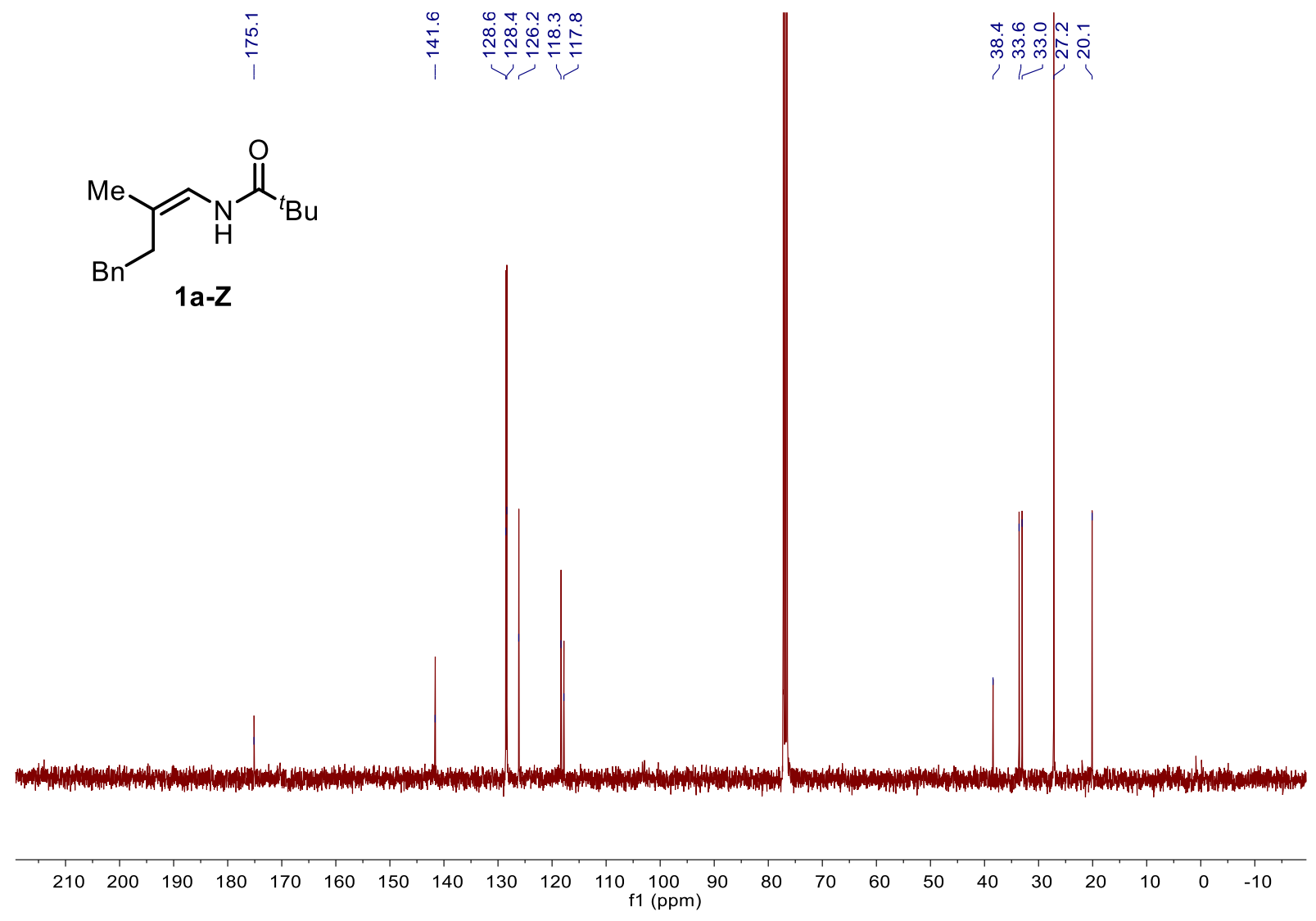

Figure S53. ${ }^{13} \mathbf{C}$ NMR $\left(101 \mathrm{MHz}, \mathrm{CDCl}_{3}\right)$ of $\mathbf{1 a - Z}$ 
<smiles>[Y19]C(CBr)(CBr)CNC(=O)C(C)C</smiles>

$4 a$

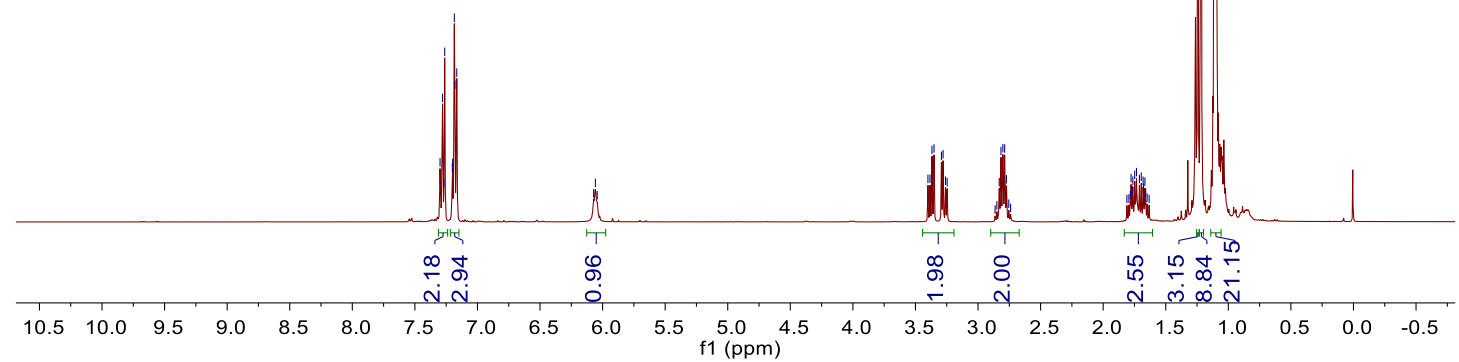

Figure S54. ${ }^{1} \mathbf{H}$ NMR $\left(400 \mathrm{MHz}, \mathrm{CDCl}_{3}\right)$ of $\mathbf{4 a}$

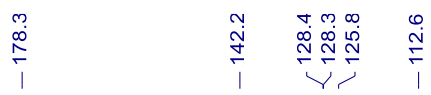

\section{$\underset{\substack{0 \\ \infty \\ 1 \\ 1}}{\substack{j \\ 1}}$}

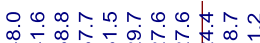

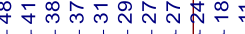<smiles>CC(C)CC(=O)NC[C@@](C)(C#CSC(C)C)CBr</smiles>

$4 a$

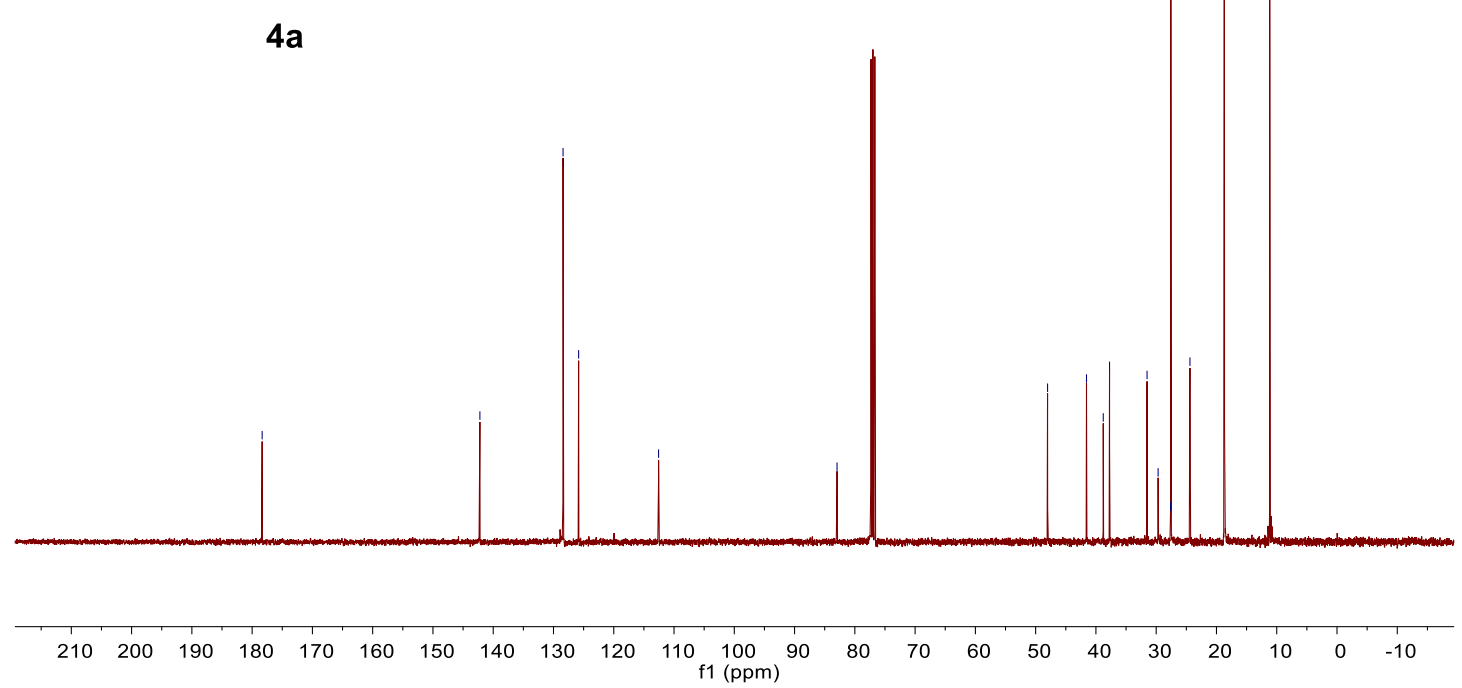

Figure S55. ${ }^{13} \mathbf{C}$ NMR $\left(101 \mathrm{MHz}, \mathrm{CDCl}_{3}\right)$ of $\mathbf{4 a}$ 


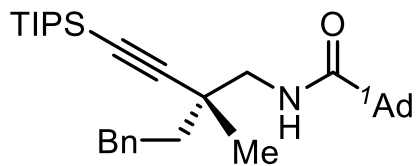

4b

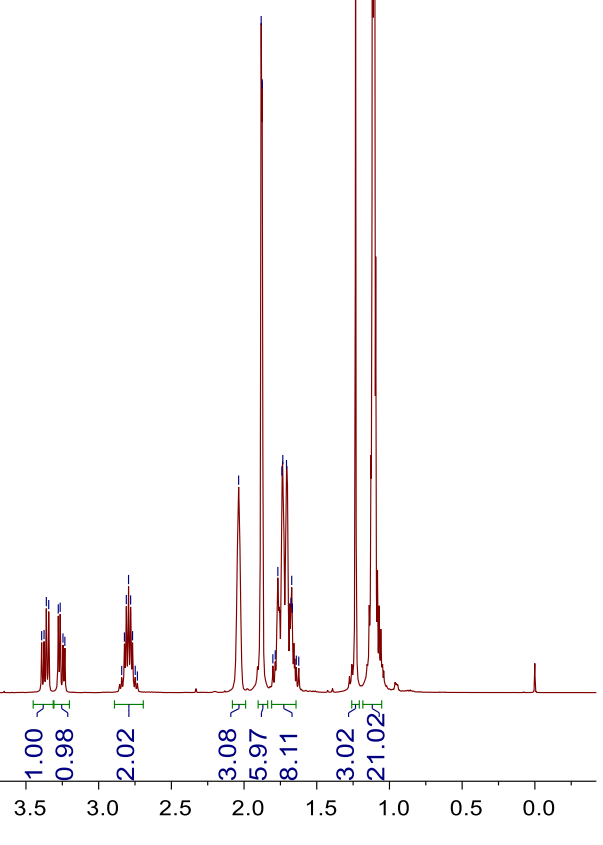

Figure S56. ${ }^{\mathbf{1}} \mathbf{H}$ NMR $\left(400 \mathrm{MHz}, \mathrm{CDCl}_{3}\right)$ of $\mathbf{4 b}$

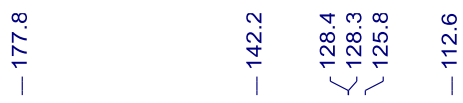

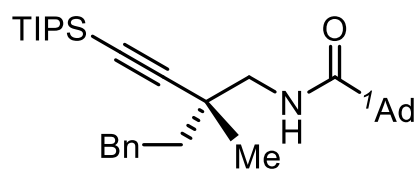

4b

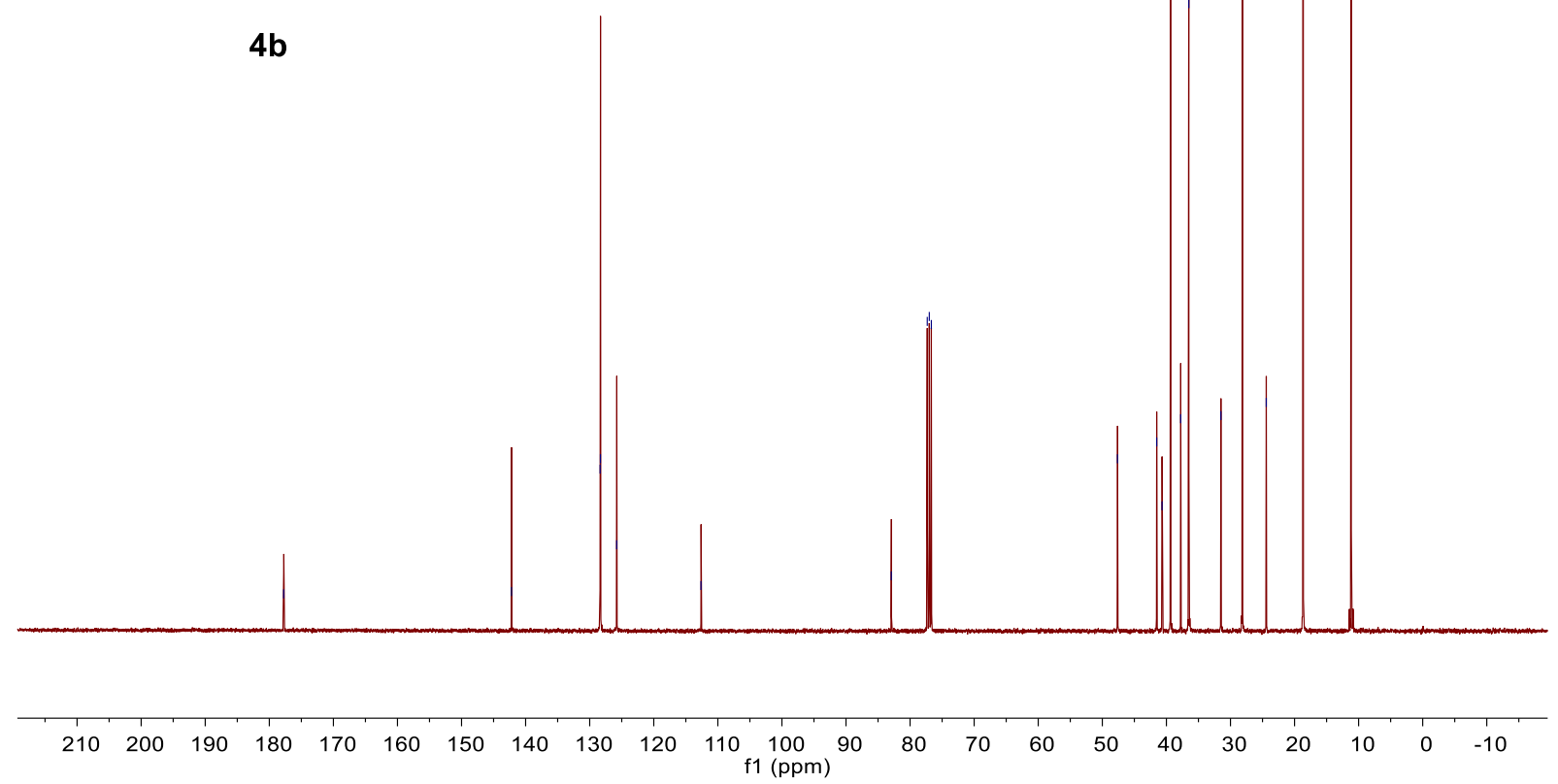

Figure S57. ${ }^{13} \mathbf{C}$ NMR $\left(101 \mathrm{MHz}, \mathrm{CDCl}_{3}\right)$ of $\mathbf{4 b}$ 


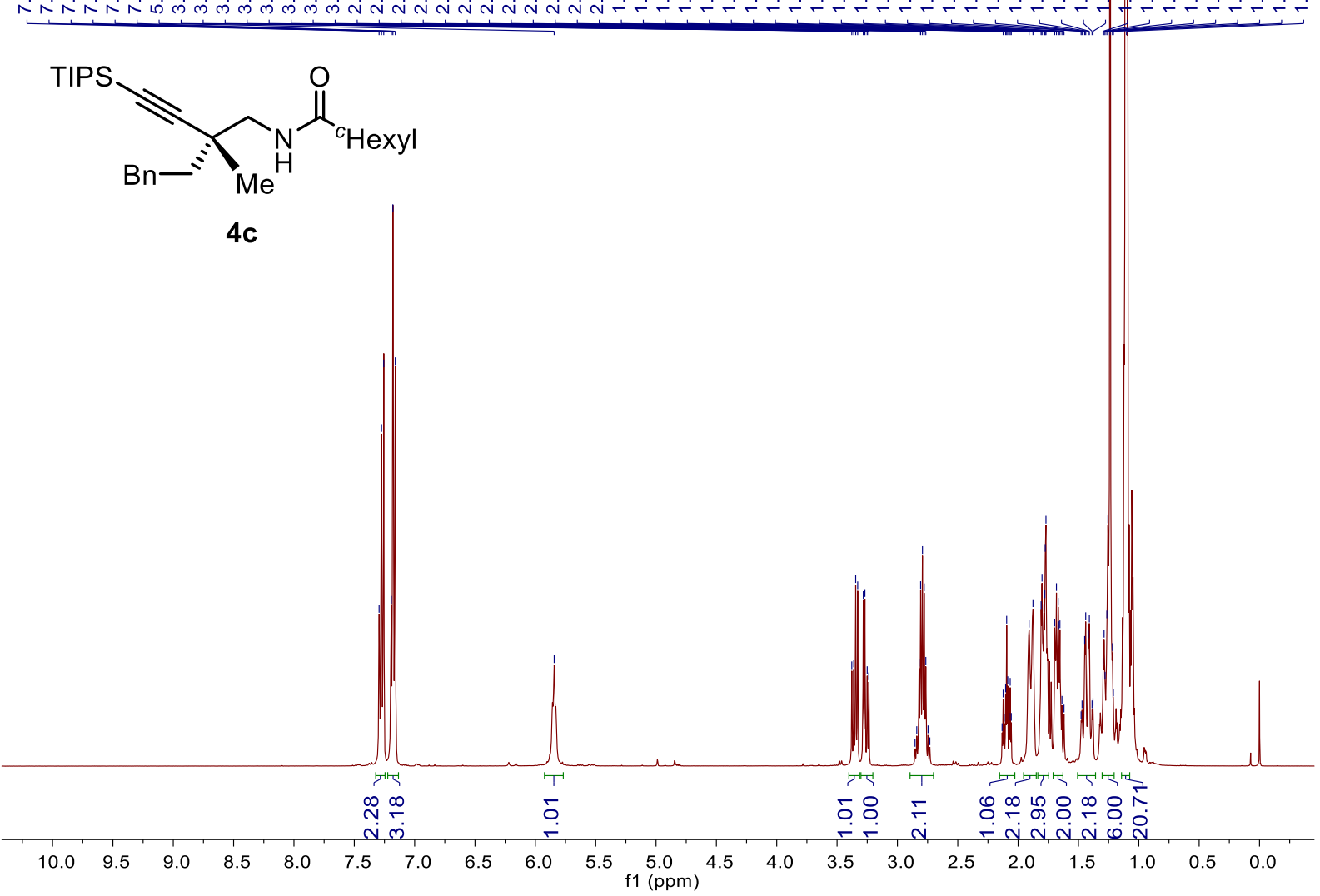

Figure S58. ${ }^{1} \mathbf{H}$ NMR $\left(400 \mathrm{MHz}, \mathrm{CDCl}_{3}\right)$ of $\mathbf{4 c}$

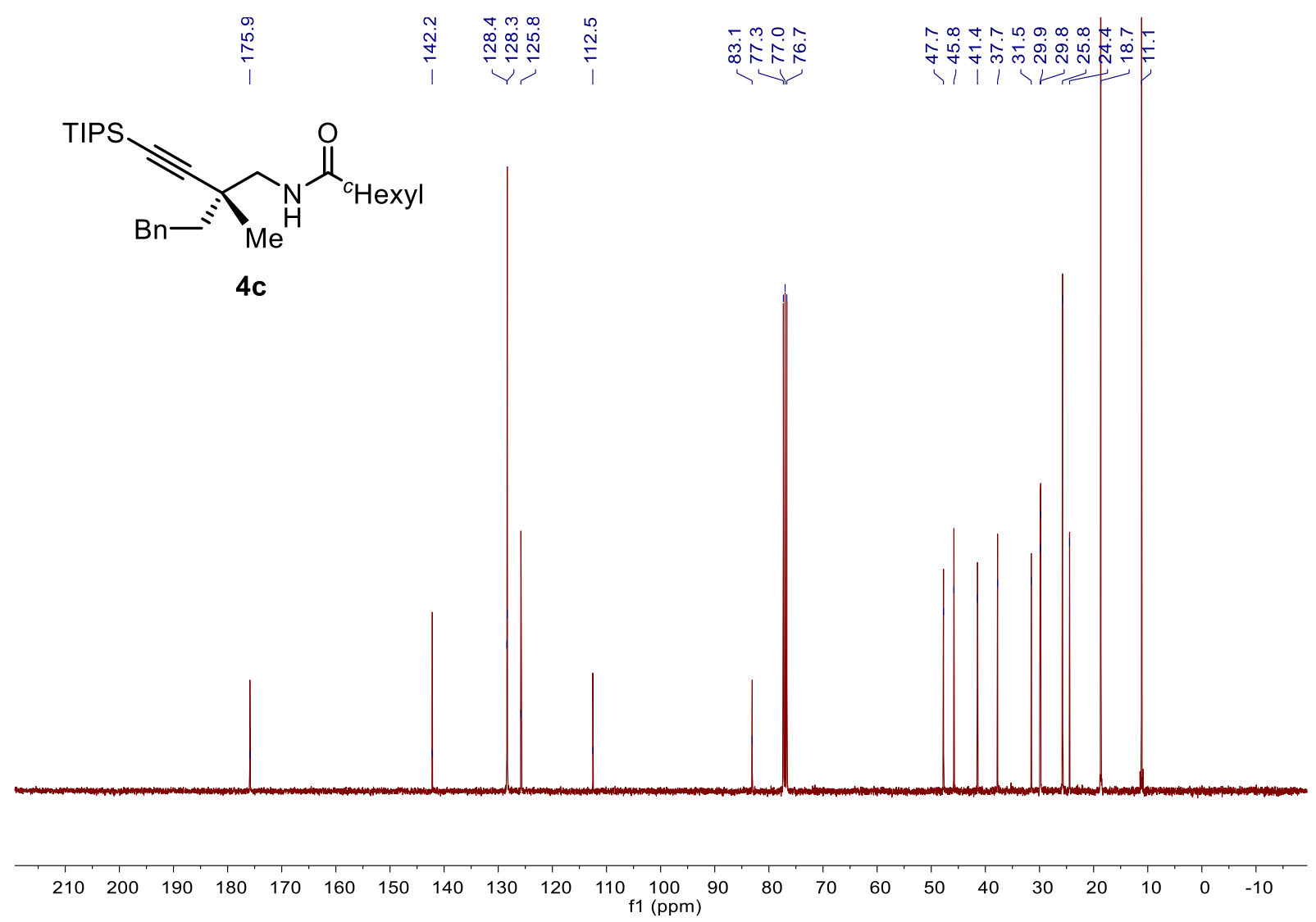

Figure S59. ${ }^{13} \mathbf{C}$ NMR $\left(101 \mathrm{MHz}, \mathrm{CDCl}_{3}\right)$ of $\mathbf{4 c}$ 
<smiles>[M][C@@](C#CS[In])(CBr)CNC(=O)CCC</smiles>

$4 d$

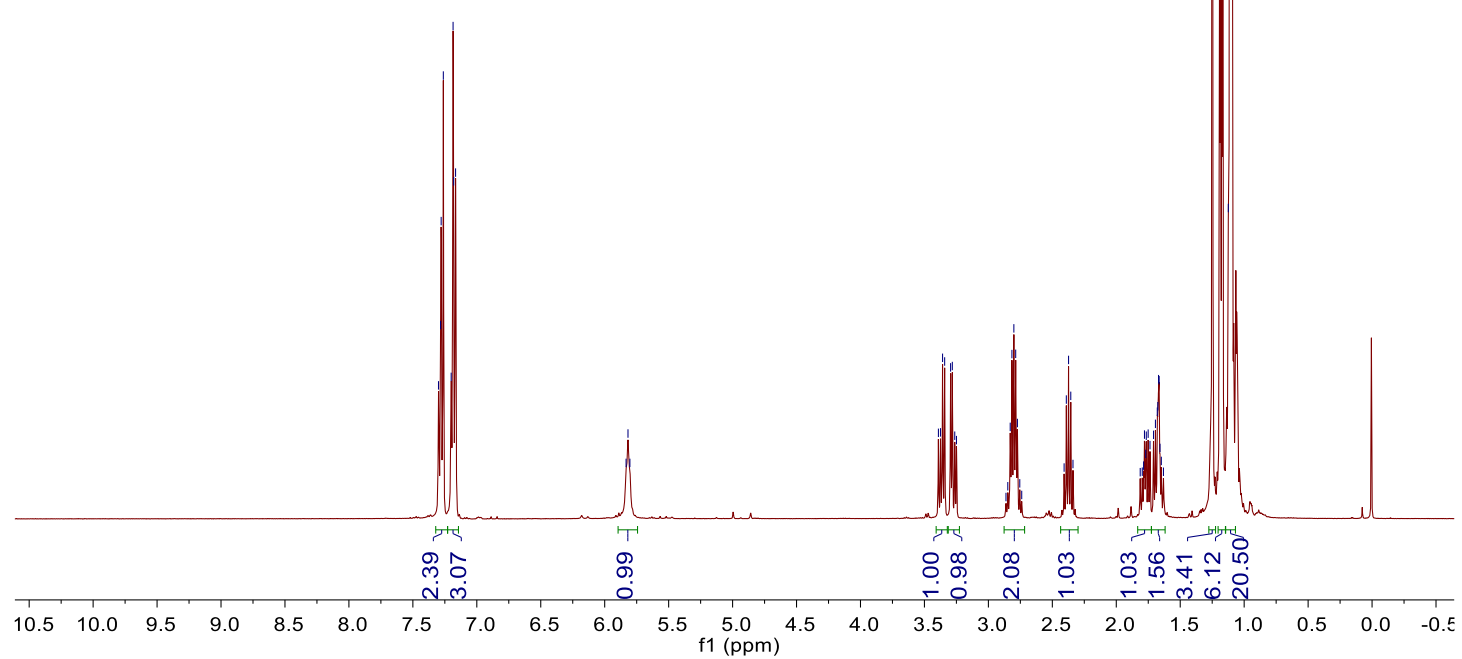

Figure S60. ${ }^{1} \mathbf{H}$ NMR $\left(400 \mathrm{MHz}, \mathrm{CDCl}_{3}\right)$ of $\mathbf{4 d}$

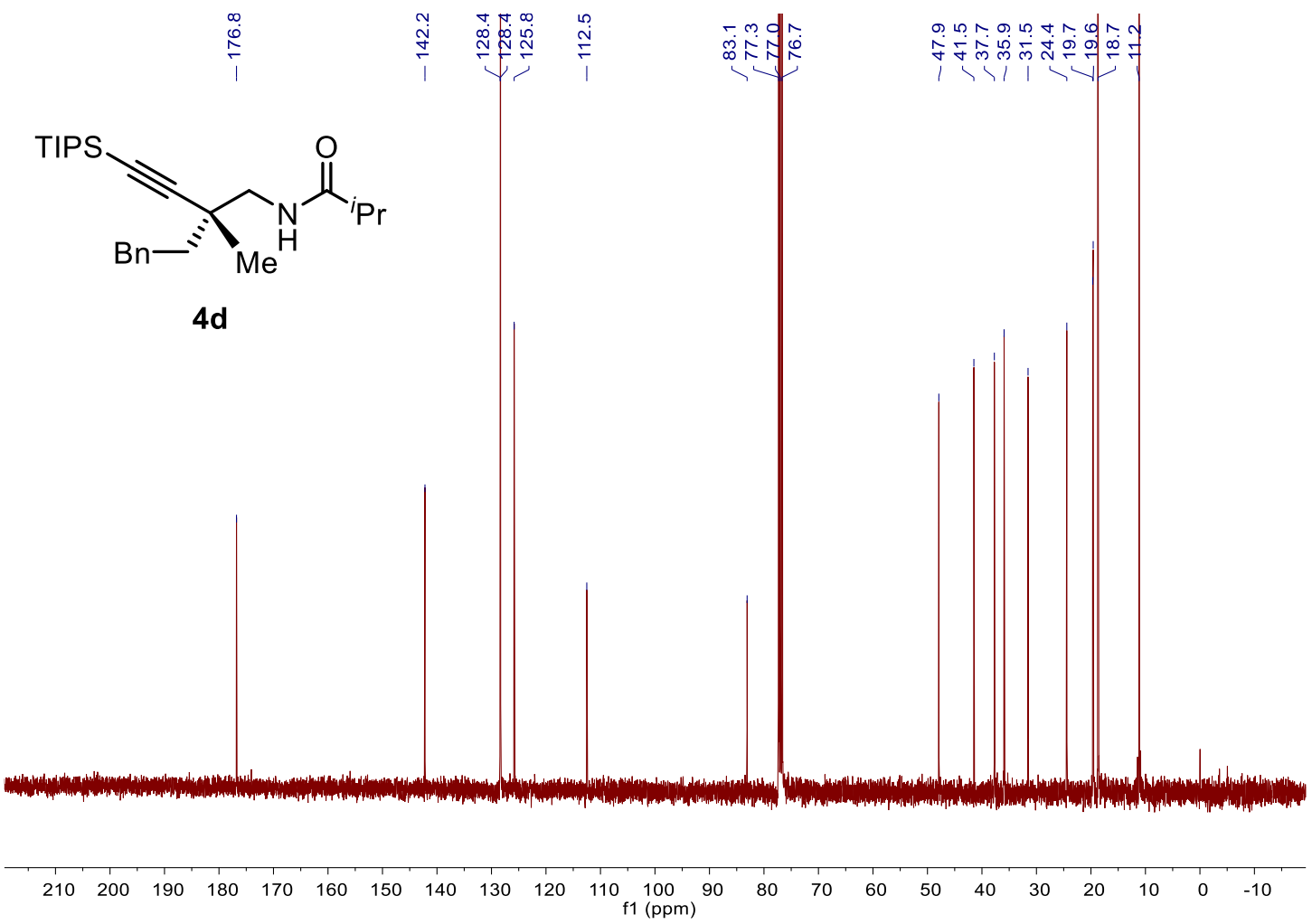

Figure S61. ${ }^{13} \mathbf{C}$ NMR $\left(101 \mathrm{MHz}, \mathrm{CDCl}_{3}\right)$ of $\mathbf{4 d}$ 


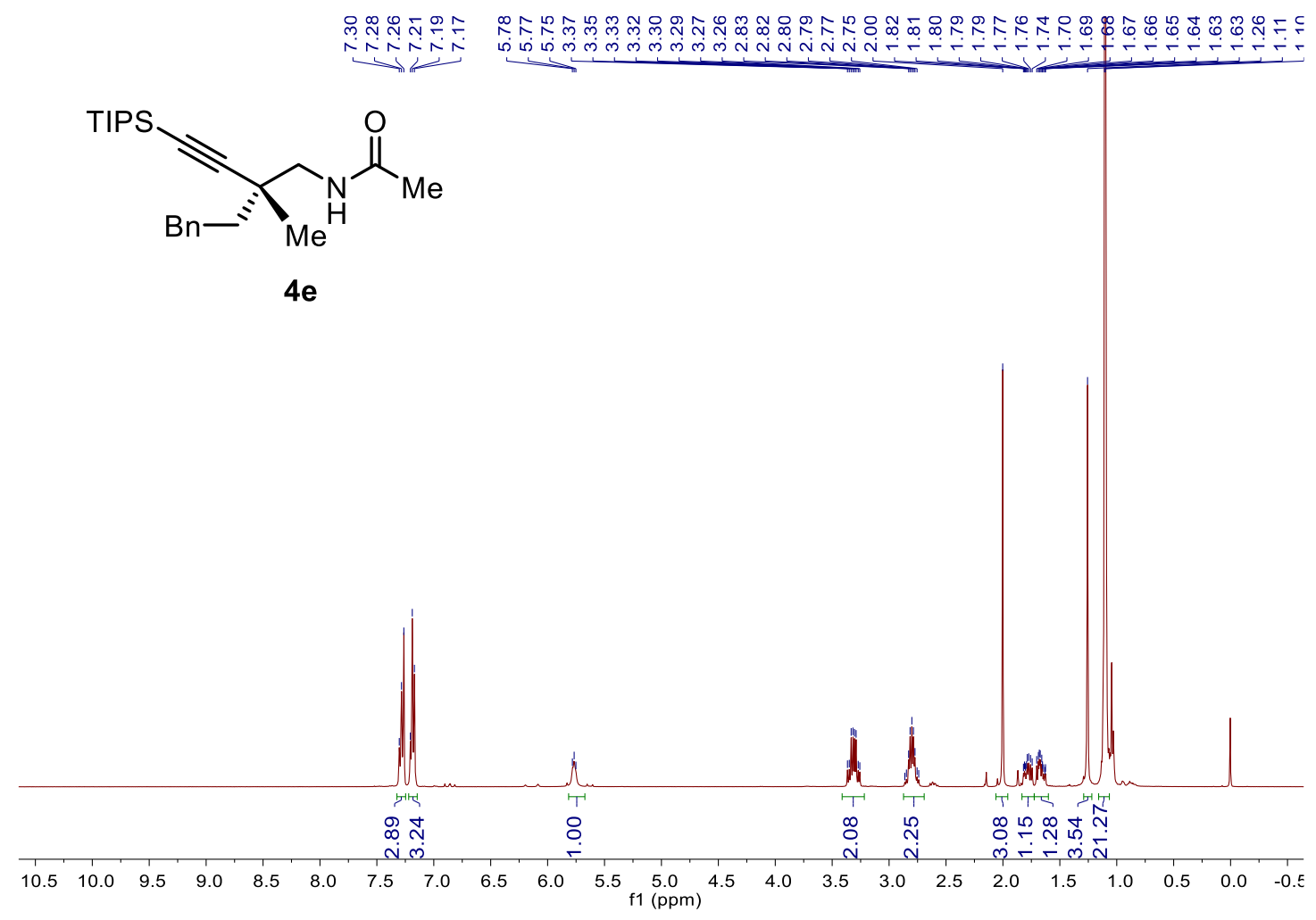

Figure S62. ${ }^{\mathbf{1}} \mathbf{H}$ NMR $\left(400 \mathrm{MHz}, \mathrm{CDCl}_{3}\right)$ of $\mathbf{4 e}$

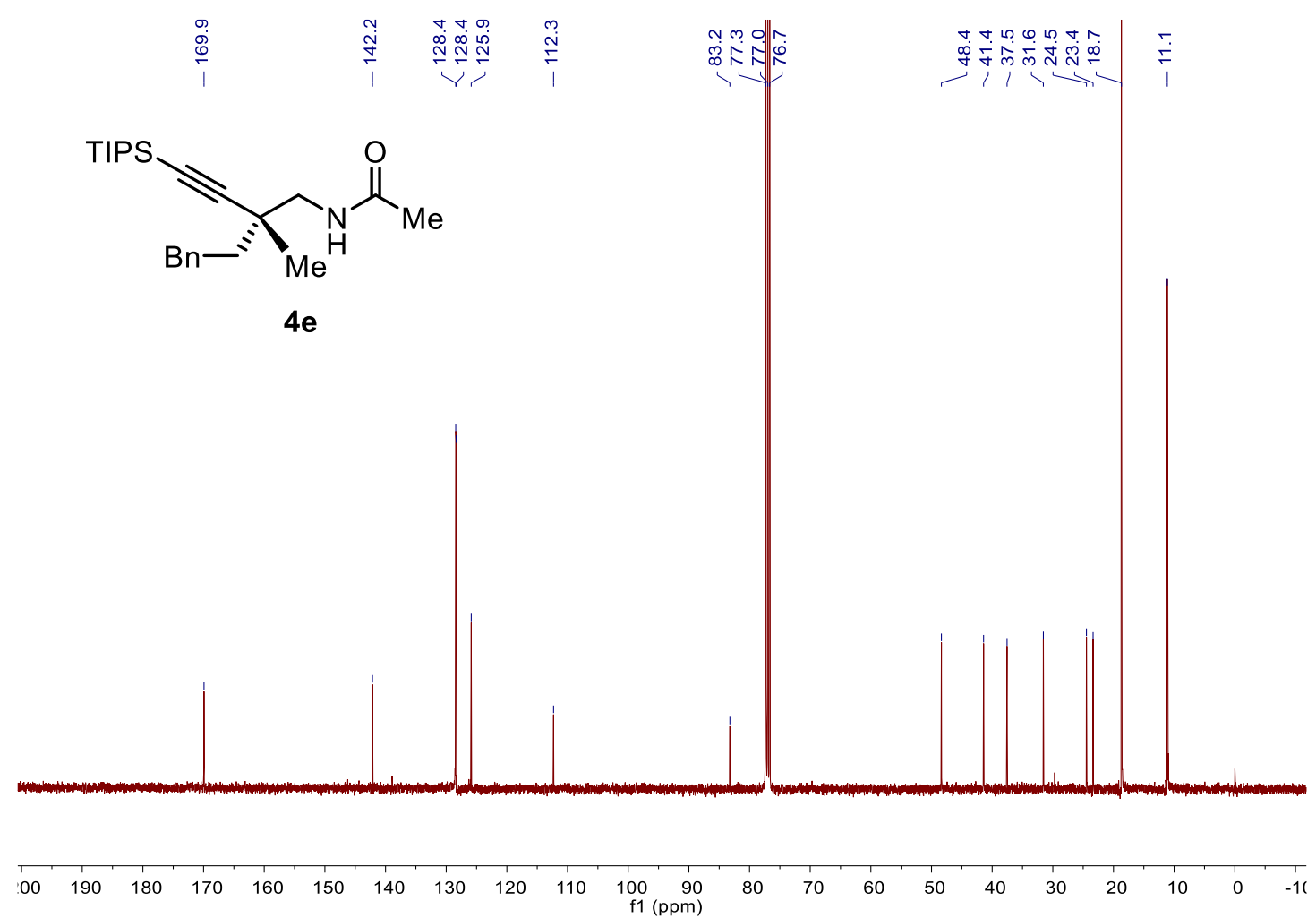

Figure S63. ${ }^{13} \mathbf{C}$ NMR $\left(101 \mathrm{MHz}, \mathrm{CDCl}_{3}\right)$ of $\mathbf{4 e}$ 


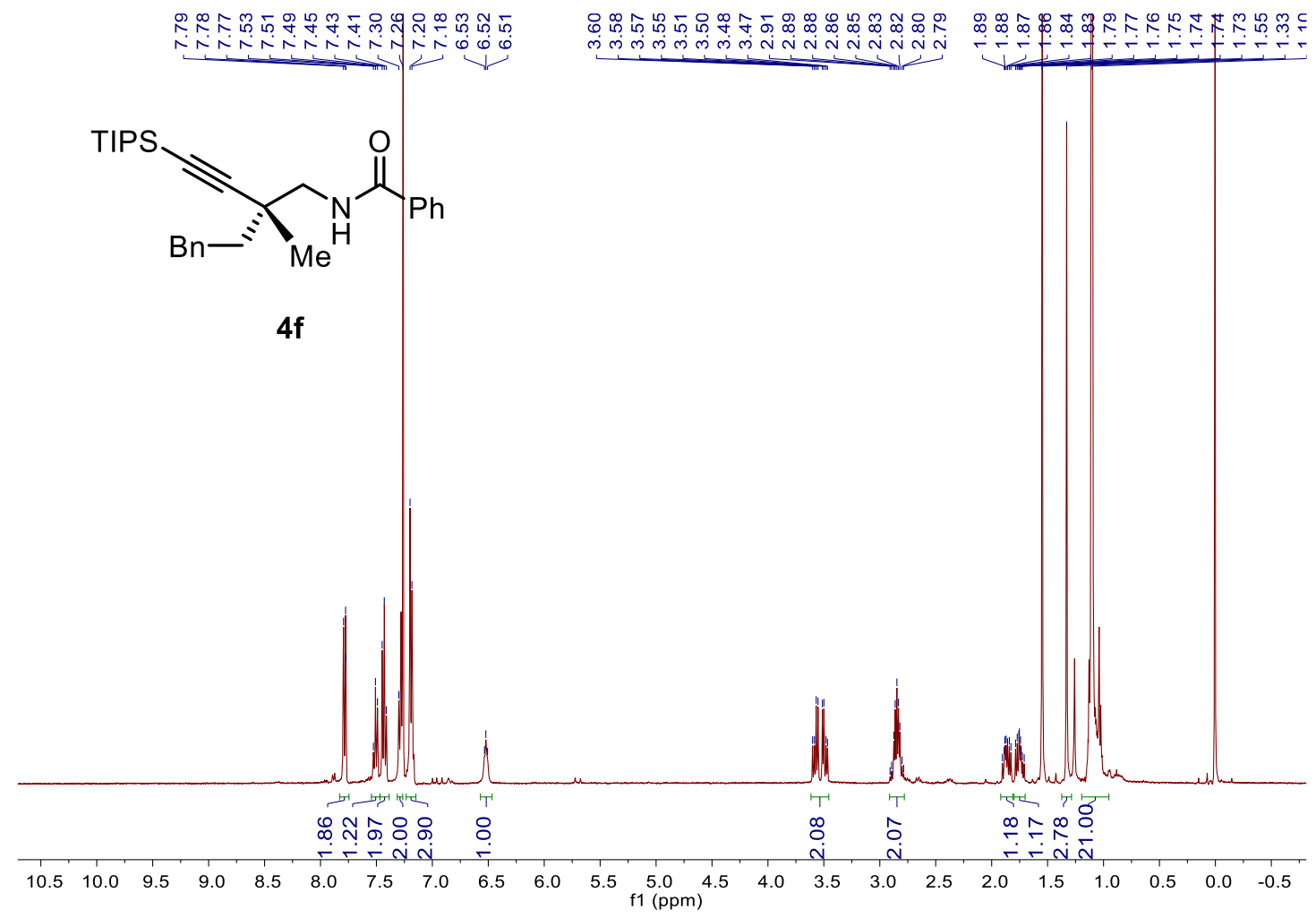

Figure S64. ${ }^{1} \mathbf{H}$ NMR $\left(400 \mathrm{MHz}, \mathrm{CDCl}_{3}\right)$ of $\mathbf{4 f}$<smiles>CCCNC(=O)c1ccccc1</smiles>

$4 f$

Figure S65. ${ }^{13} \mathbf{C}$ NMR $\left(101 \mathrm{MHz}, \mathrm{CDCl}_{3}\right)$ of $\mathbf{4 f}$ 


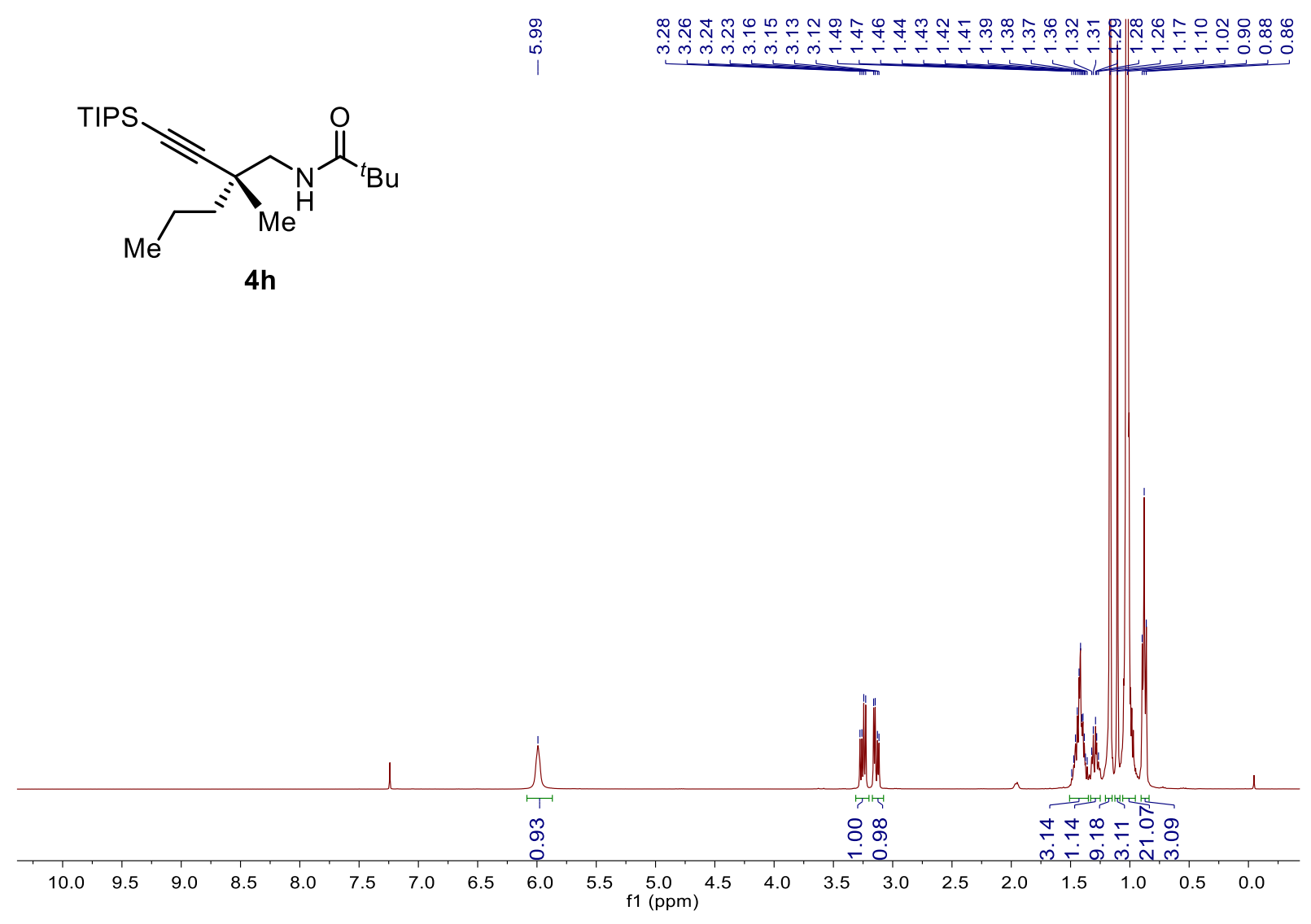

Figure S66. ${ }^{\mathbf{1}} \mathbf{H}$ NMR $\left(400 \mathrm{MHz}, \mathrm{CDCl}_{3}\right)$ of $\mathbf{4 h}$

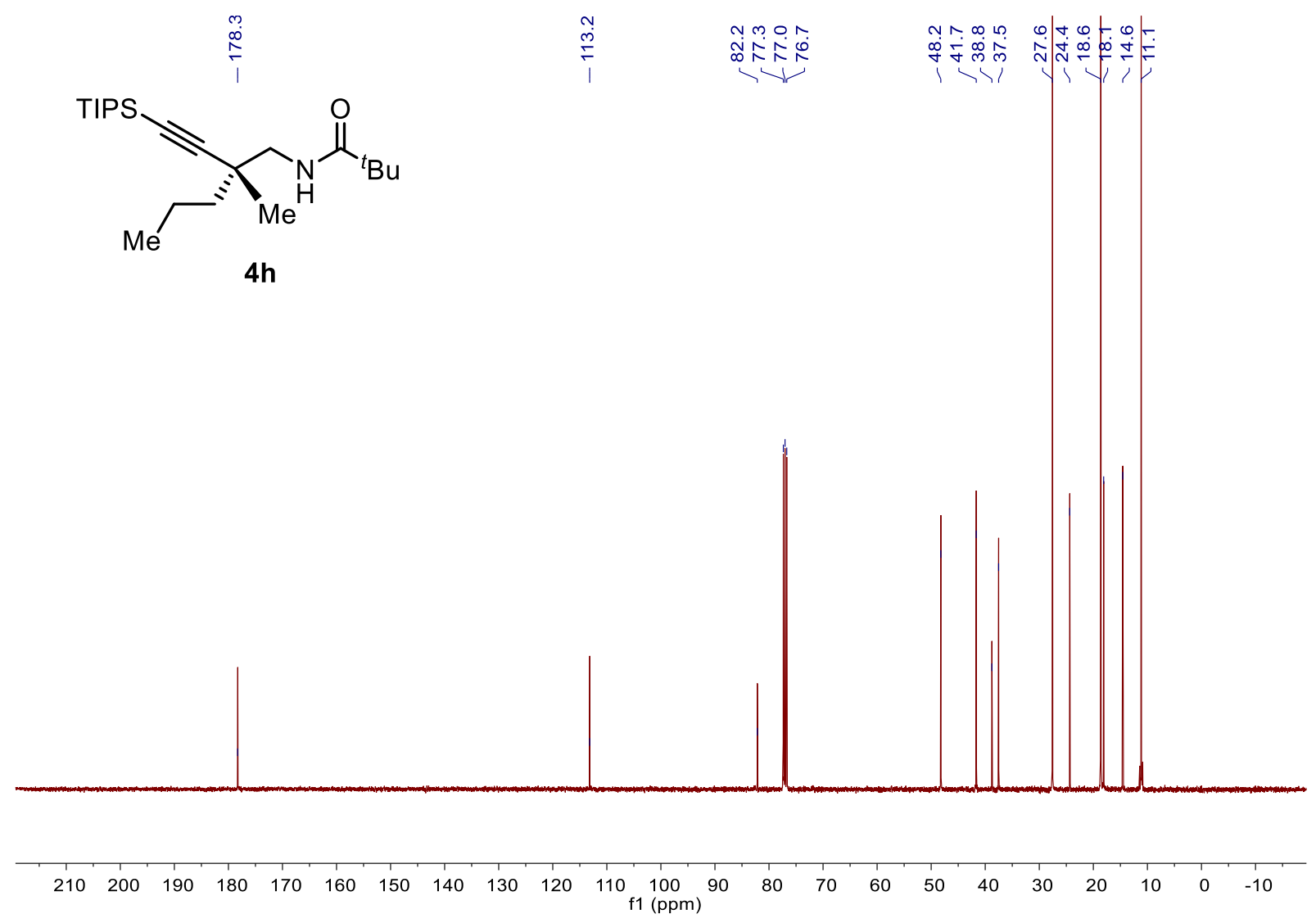

Figure S67. ${ }^{13} \mathbf{C}$ NMR $\left(101 \mathrm{MHz}, \mathrm{CDCl}_{3}\right)$ of $\mathbf{4 h}$ 


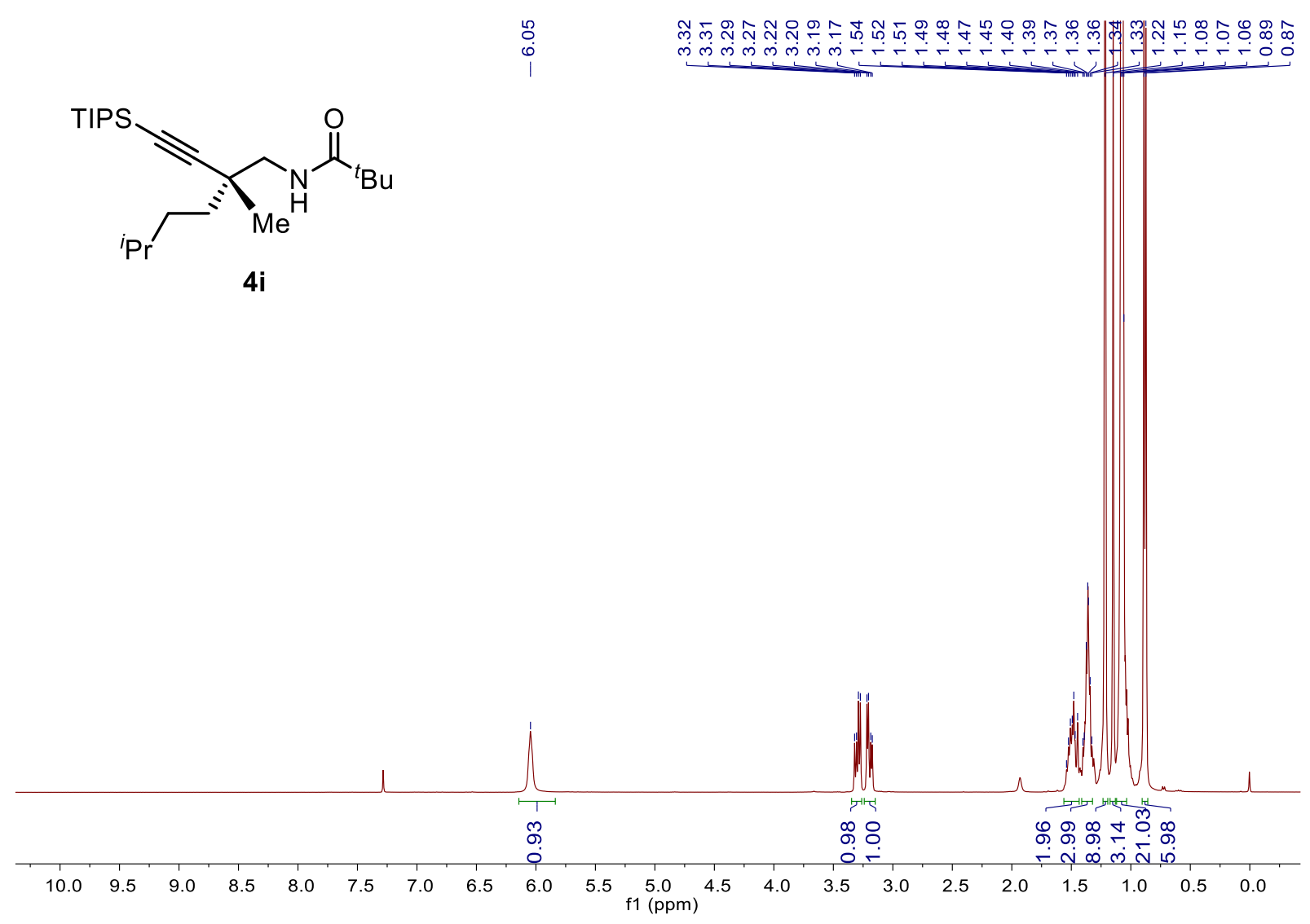

Figure S68. ${ }^{1} \mathbf{H}$ NMR $\left(400 \mathrm{MHz}, \mathrm{CDCl}_{3}\right)$ of $4 \mathbf{i}$

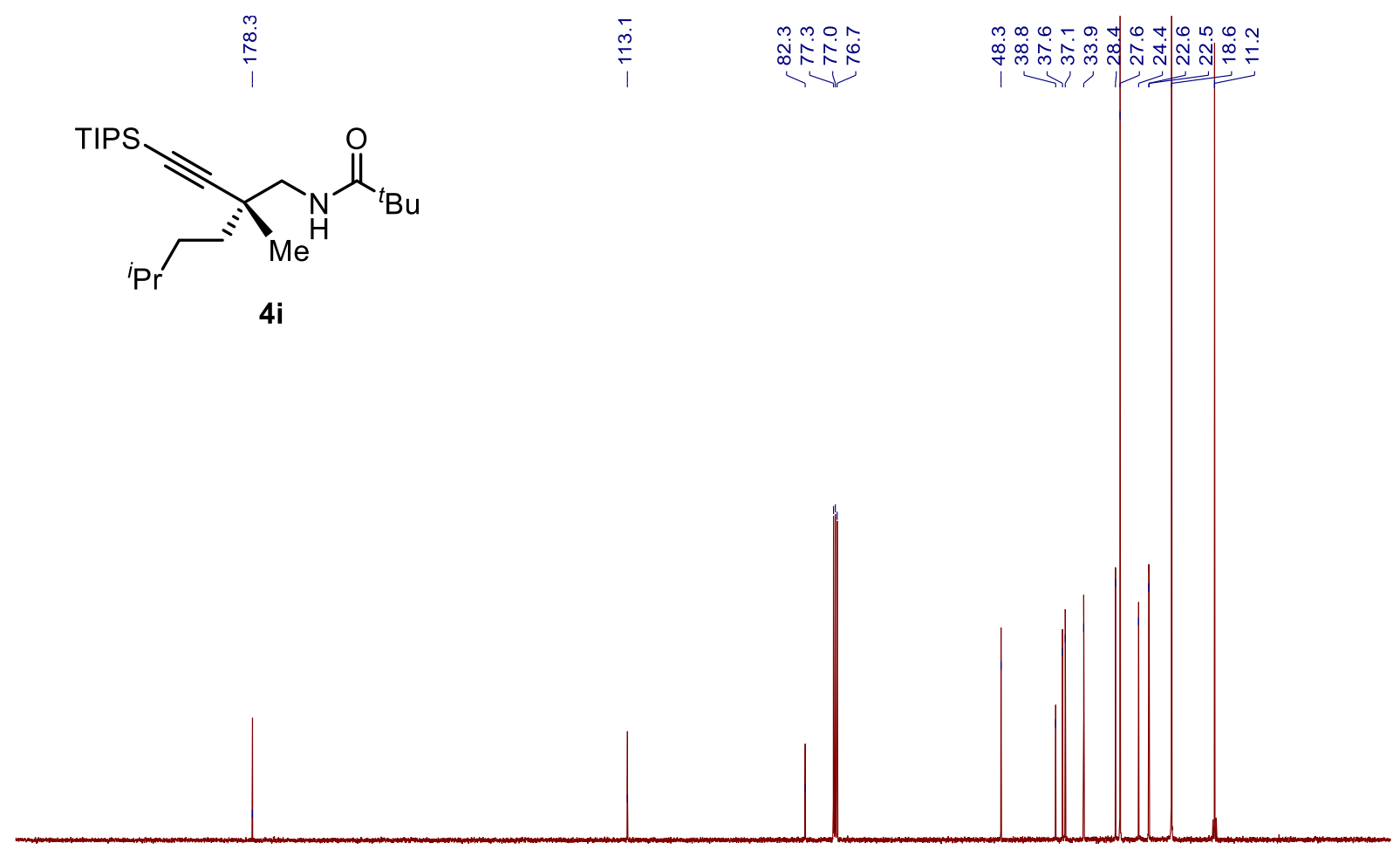

$\begin{array}{llllllllllllllllllllllllllllll}210 & 200 & 190 & 180 & 170 & 160 & 150 & 140 & 130 & 120 & 110 & 100 & 90 & 80 & 70 & 60 & 50 & 40 & 30 & 20 & 10 & 0 & -10\end{array}$

Figure S69. ${ }^{13} \mathbf{C}$ NMR $\left(101 \mathrm{MHz}, \mathrm{CDCl}_{3}\right)$ of $\mathbf{4 i}$ 


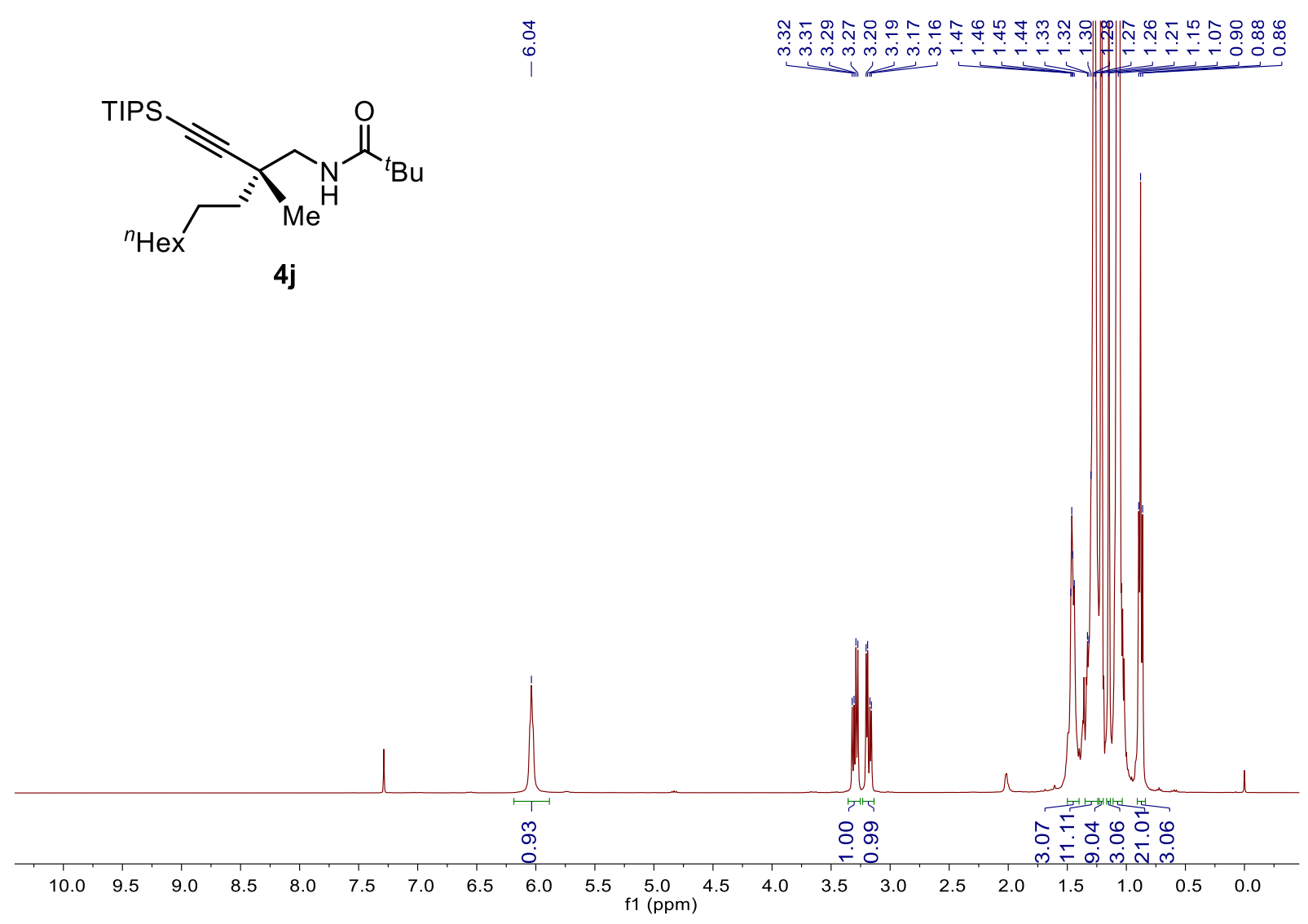

Figure S70. ${ }^{1} \mathbf{H}$ NMR $\left(400 \mathrm{MHz}, \mathrm{CDCl}_{3}\right)$ of $\mathbf{4 j}$

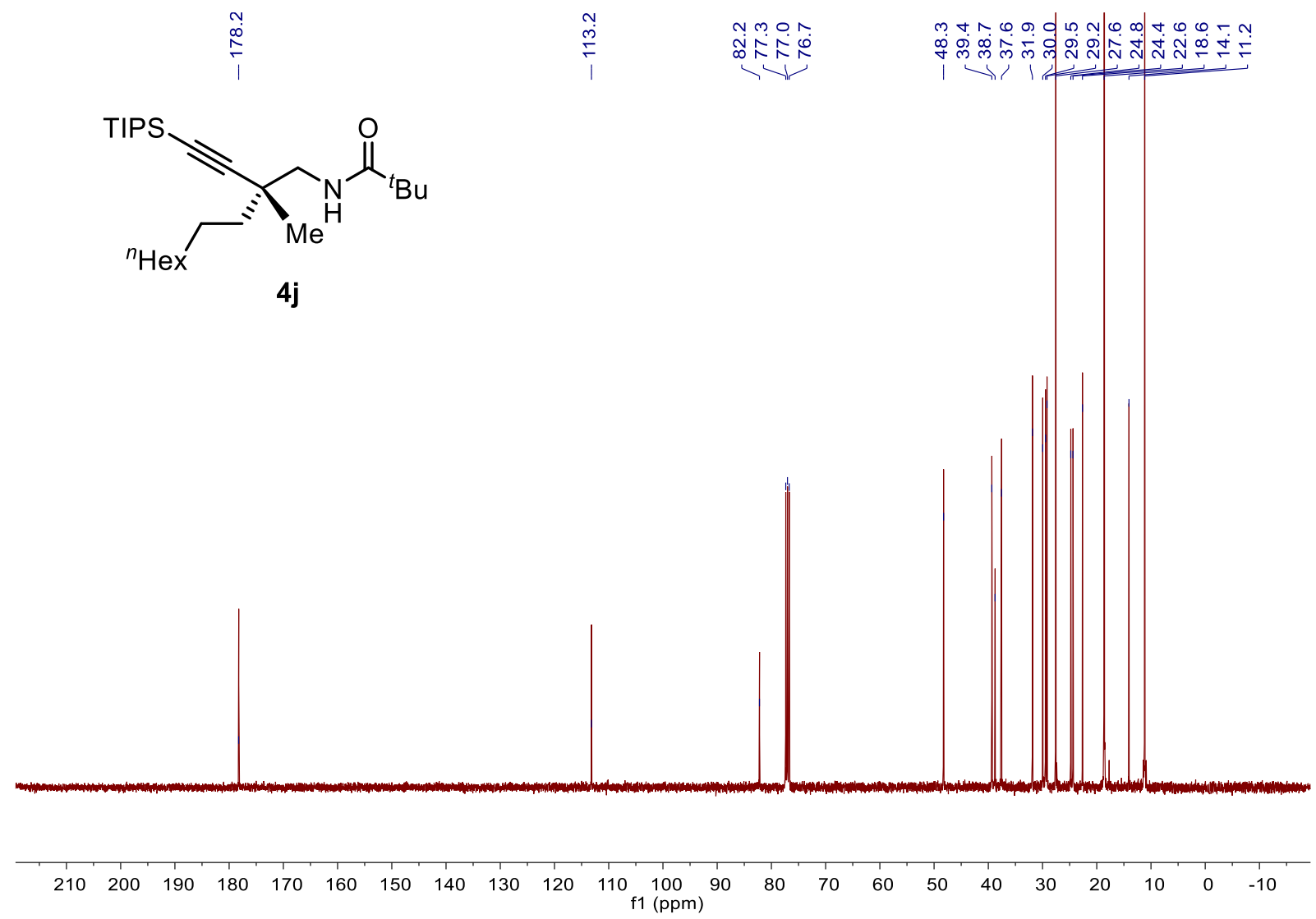

Figure S71. ${ }^{13} \mathbf{C}$ NMR $\left(101 \mathrm{MHz}, \mathrm{CDCl}_{3}\right)$ of $\mathbf{4 j}$ 


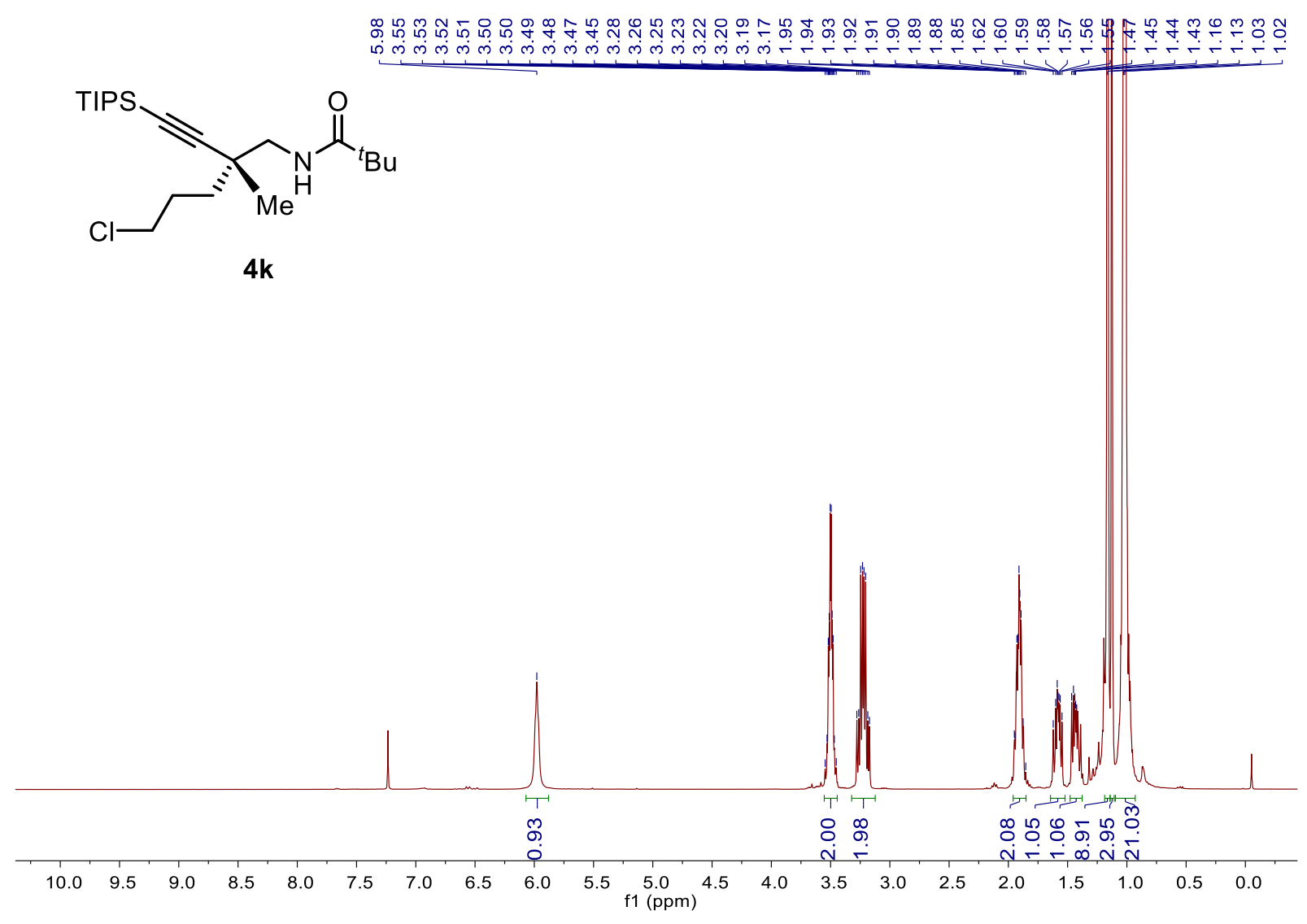

Figure S72. ${ }^{1} \mathbf{H}$ NMR $\left(400 \mathrm{MHz}, \mathrm{CDCl}_{3}\right)$ of $\mathbf{4 k}$

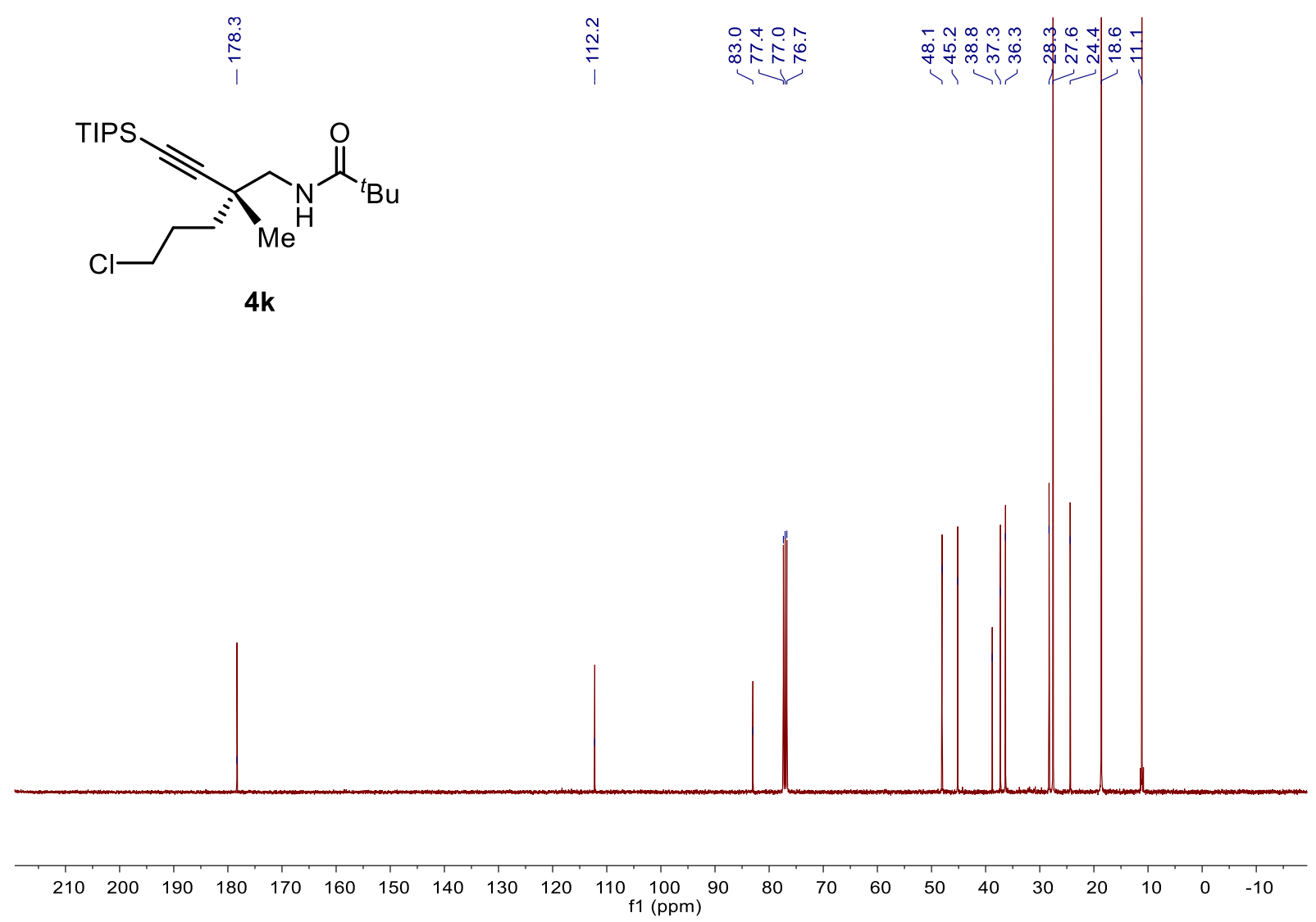

Figure S73. ${ }^{13} \mathbf{C}$ NMR $\left(101 \mathrm{MHz}, \mathrm{CDCl}_{3}\right)$ of $\mathbf{4 k}$ 


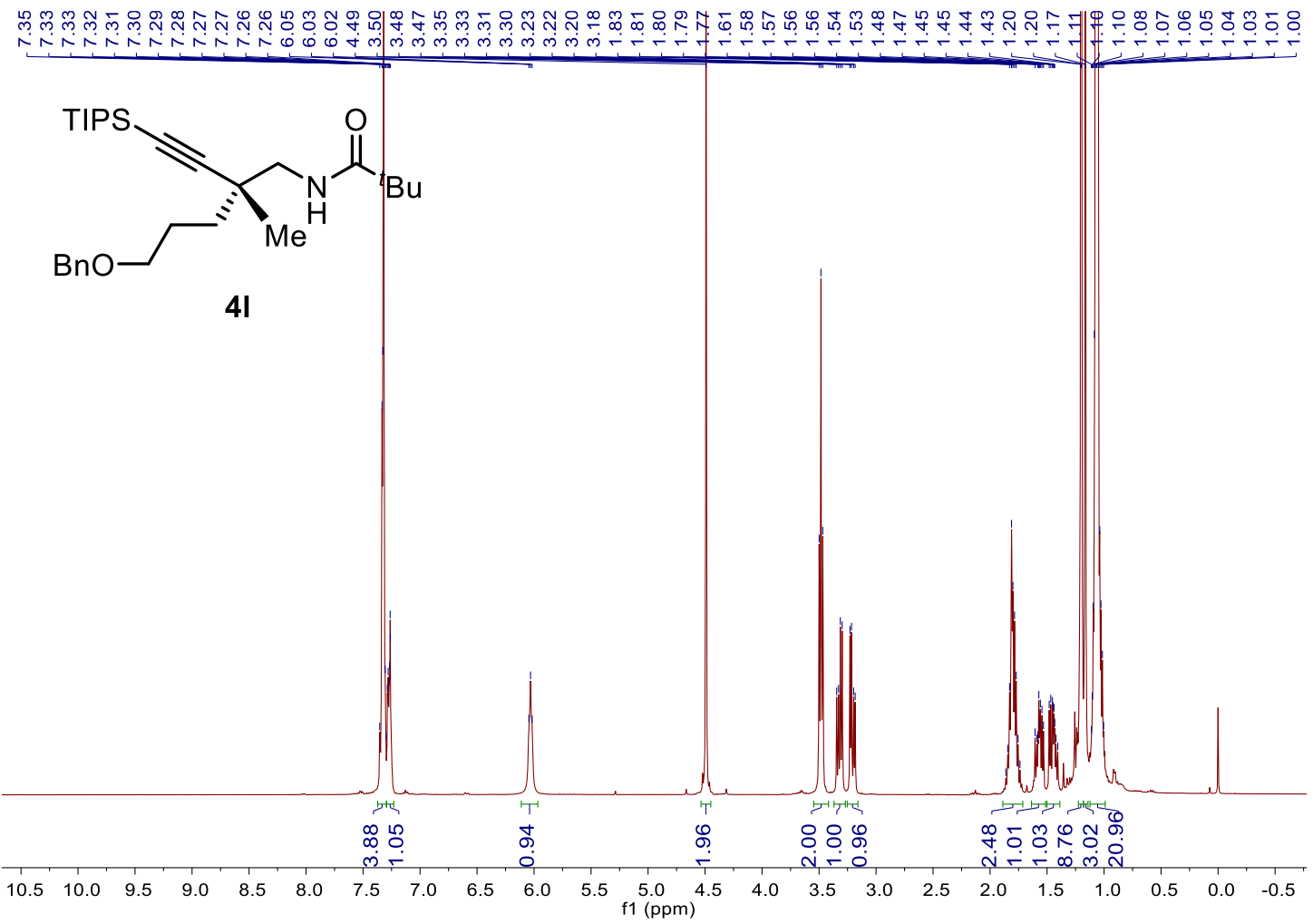

Figure S74. ${ }^{1} \mathbf{H}$ NMR $\left(400 \mathrm{MHz}, \mathrm{CDCl}_{3}\right)$ of $4 \mathrm{l}$

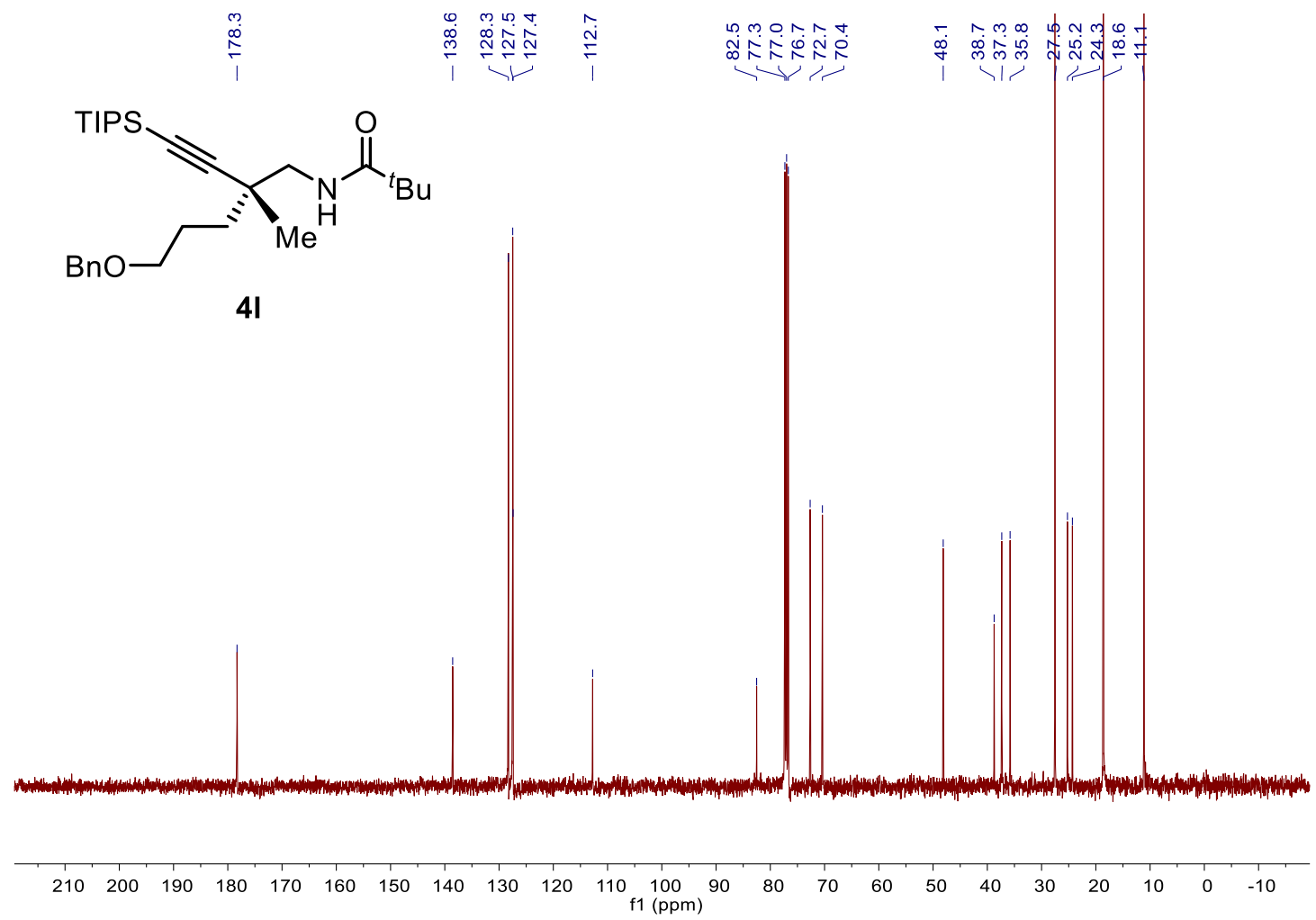

Figure S75. ${ }^{13} \mathbf{C}$ NMR $\left(101 \mathrm{MHz}, \mathrm{CDCl}_{3}\right)$ of $4 \mathbf{l}$ 


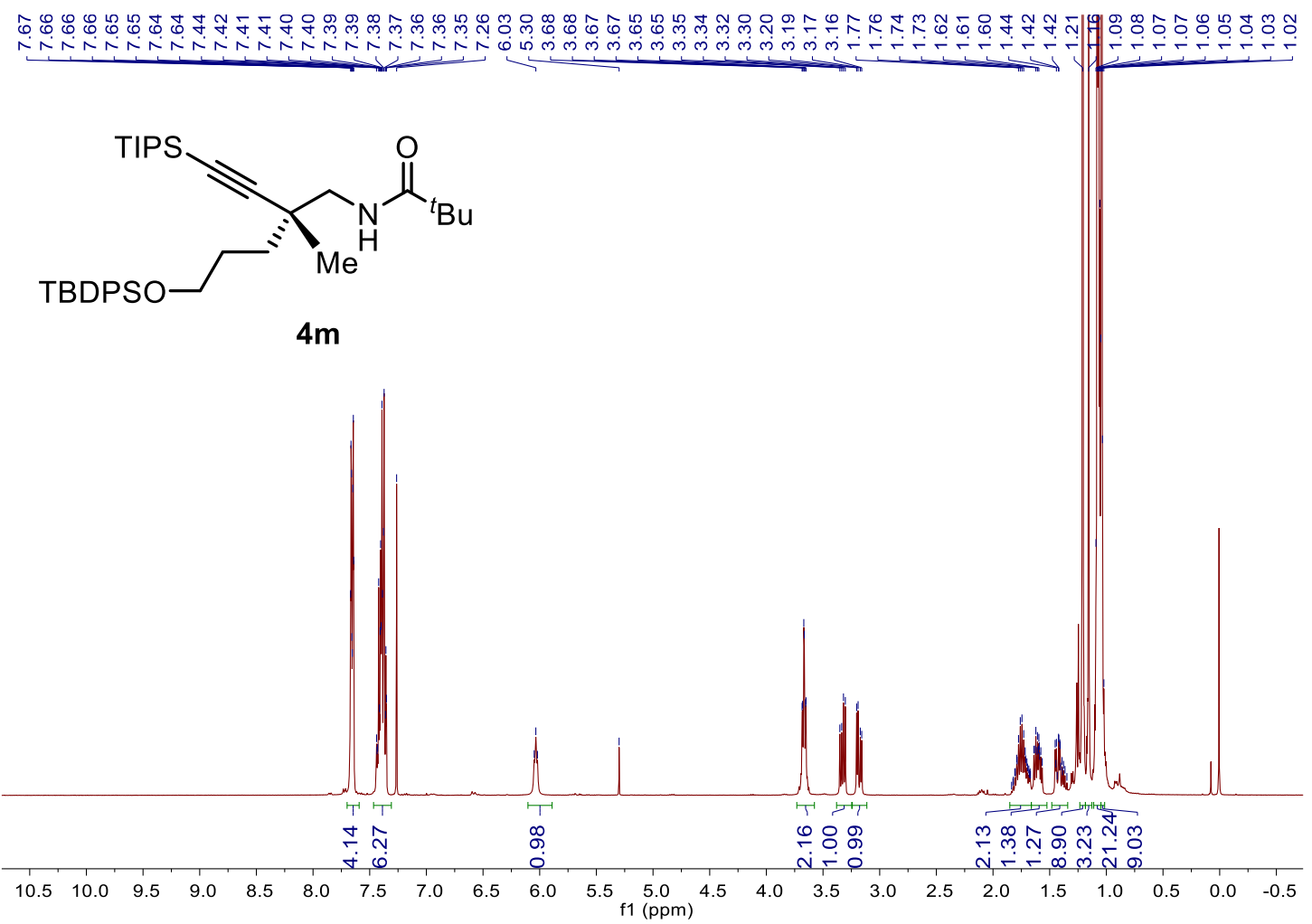

Figure S76. ${ }^{1} \mathbf{H}$ NMR $\left(400 \mathrm{MHz}, \mathrm{CDCl}_{3}\right)$ of $\mathbf{4 m}$

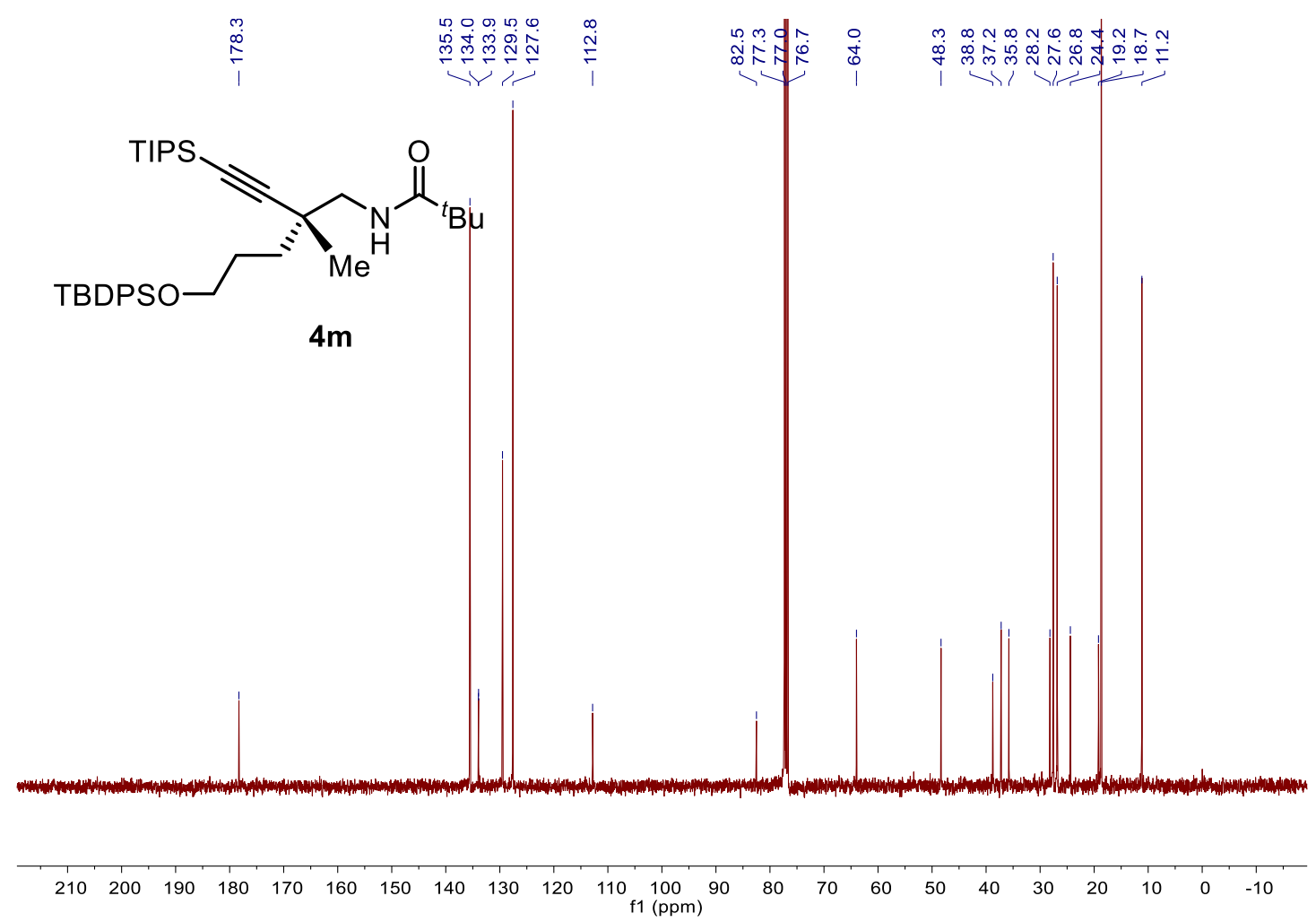

Figure S77. ${ }^{13} \mathbf{C}$ NMR $\left(101 \mathrm{MHz}, \mathrm{CDCl}_{3}\right)$ of $\mathbf{4 m}$ 


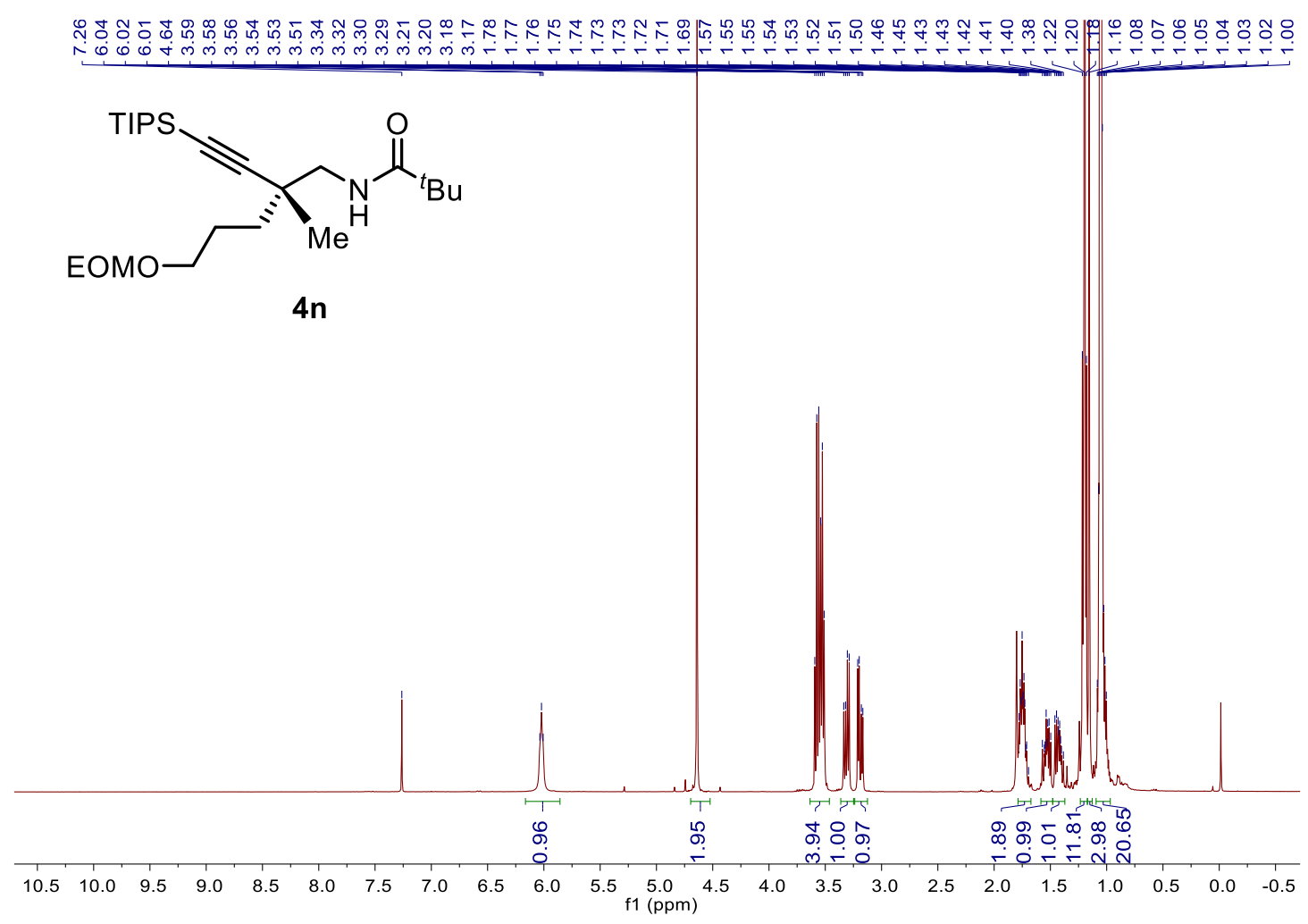

Figure S78. ${ }^{1} \mathbf{H}$ NMR $\left(400 \mathrm{MHz}, \mathrm{CDCl}_{3}\right)$ of $\mathbf{4 n}$

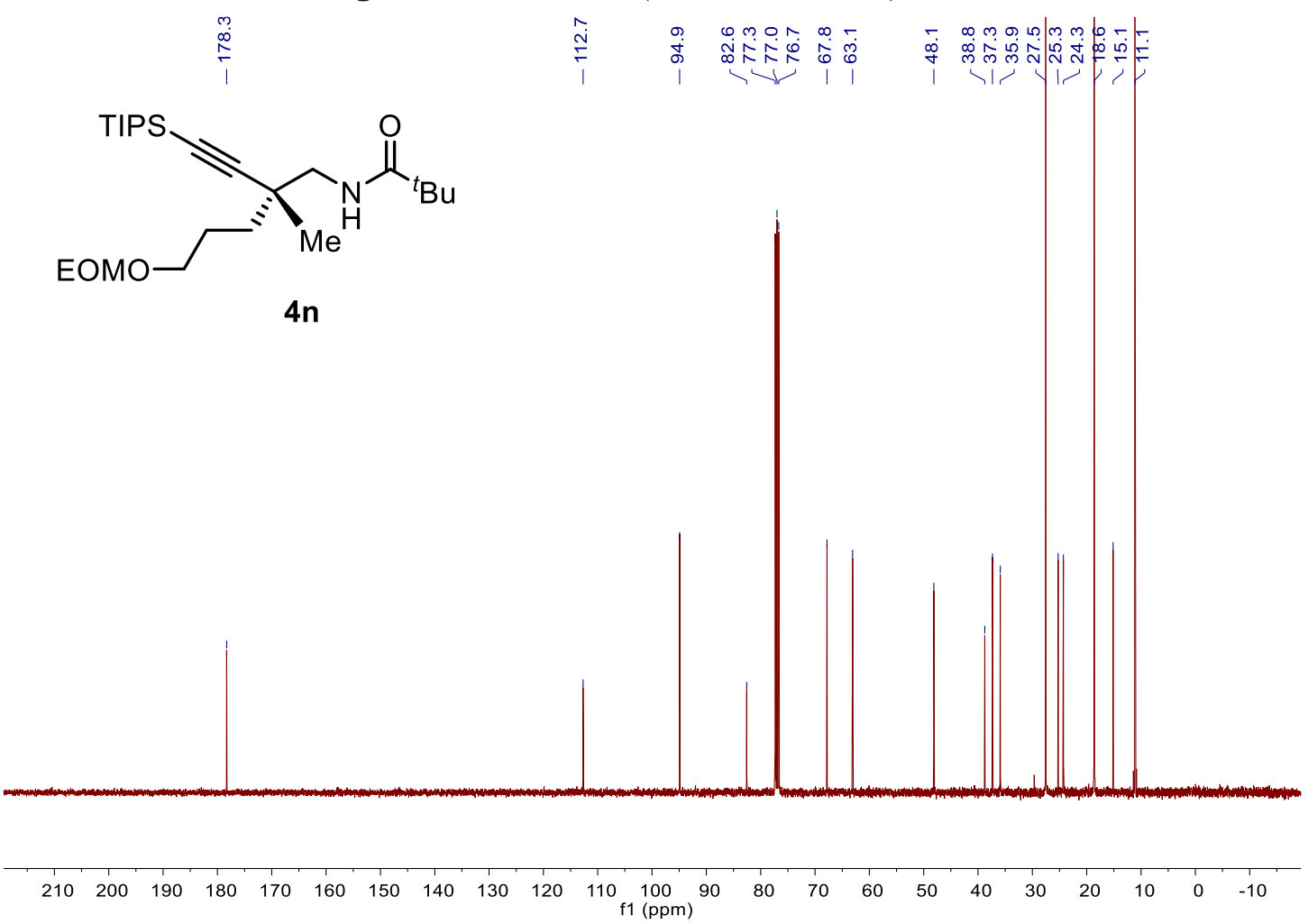

Figure S79. ${ }^{13} \mathbf{C}$ NMR $\left(101 \mathrm{MHz}, \mathrm{CDCl}_{3}\right)$ of $\mathbf{4 n}$ 


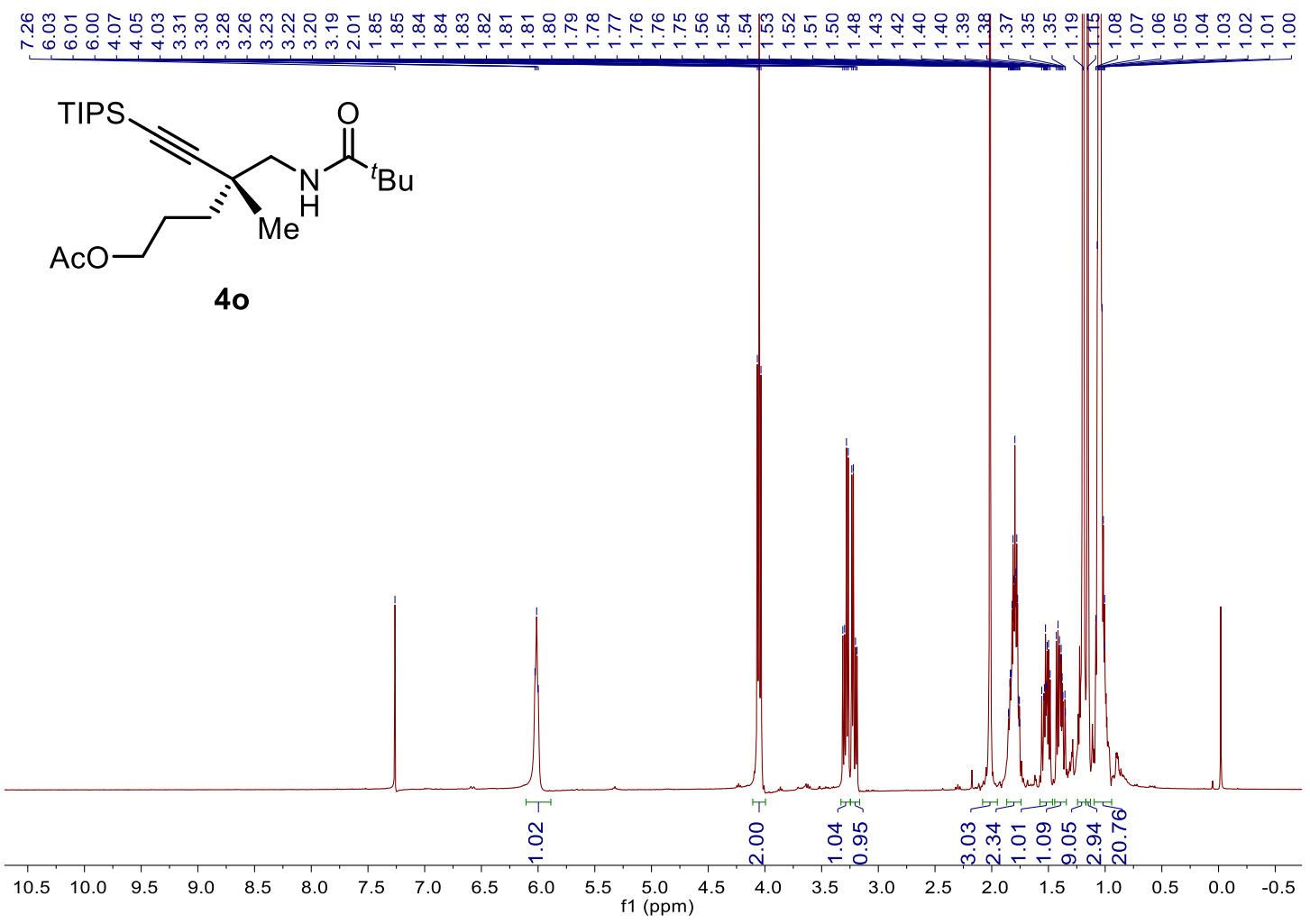

Figure S80. ${ }^{1} \mathbf{H}$ NMR $\left(400 \mathrm{MHz}, \mathrm{CDCl}_{3}\right)$ of 40

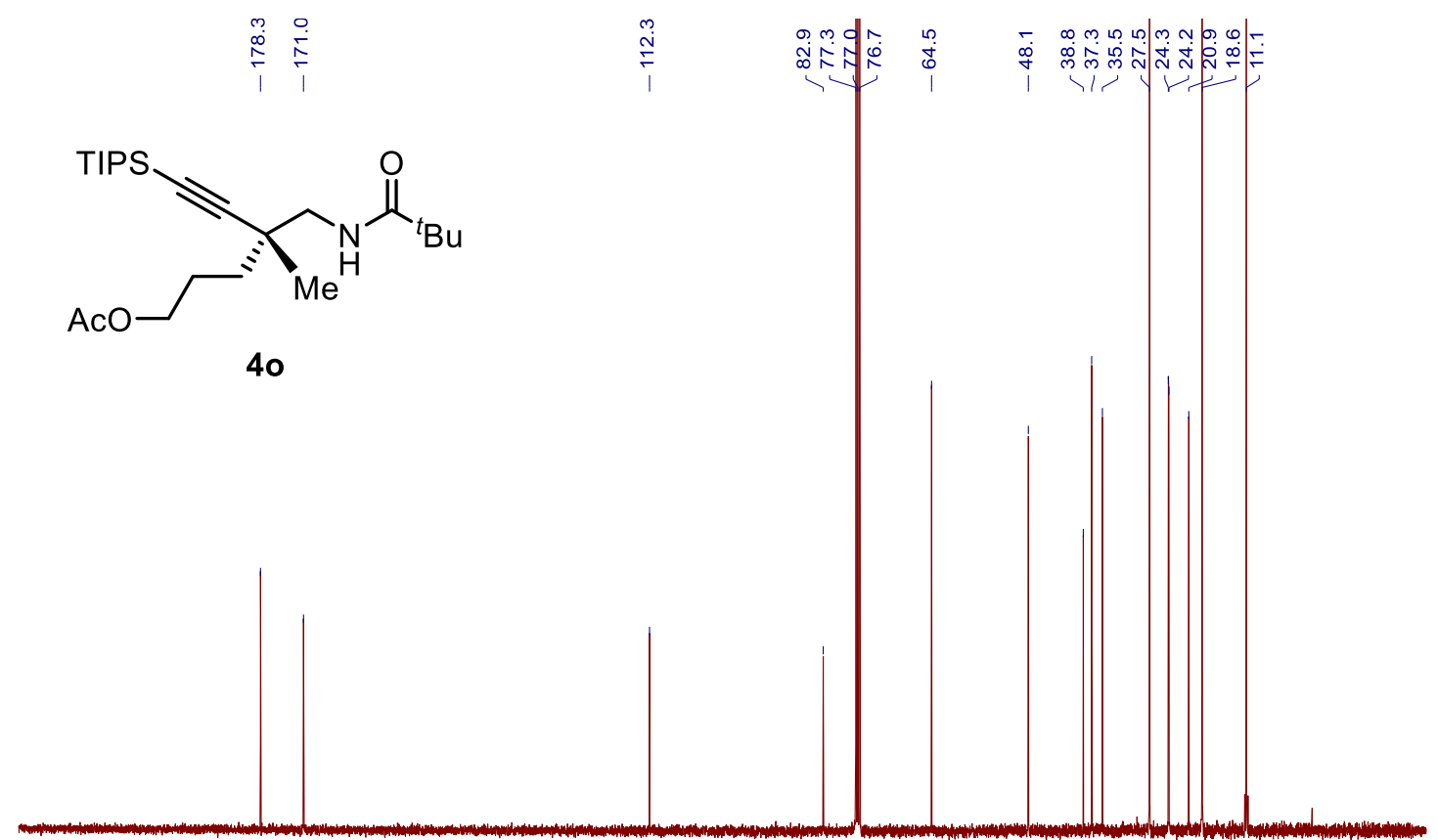

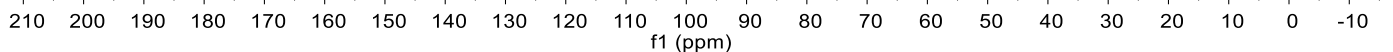

Figure S81. ${ }^{13} \mathbf{C}$ NMR $\left(101 \mathrm{MHz}, \mathrm{CDCl}_{3}\right)$ of 40 


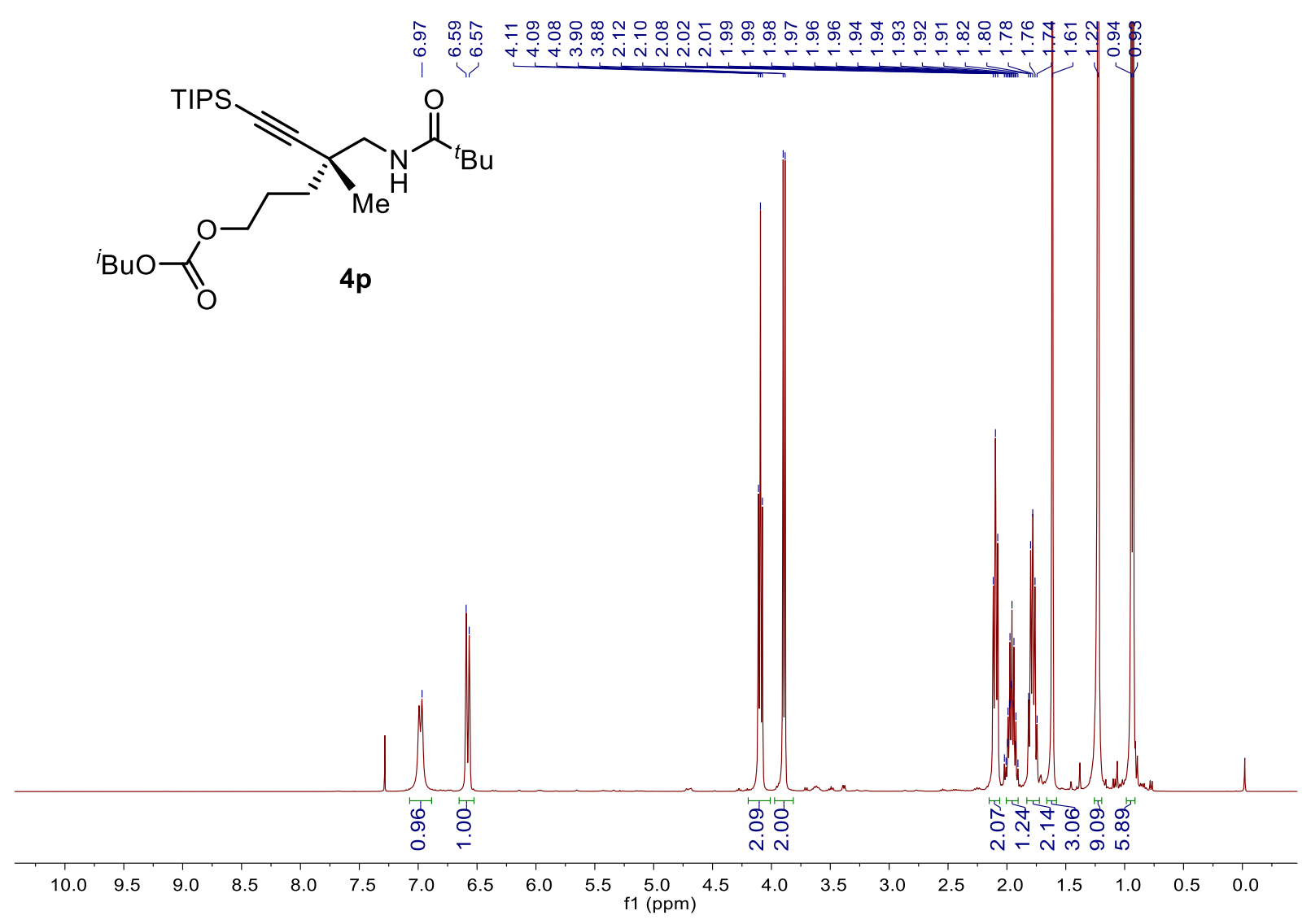

Figure S82. ${ }^{\mathbf{1}} \mathbf{H}$ NMR $\left(400 \mathrm{MHz}, \mathrm{CDCl}_{3}\right)$ of $\mathbf{4 p}$

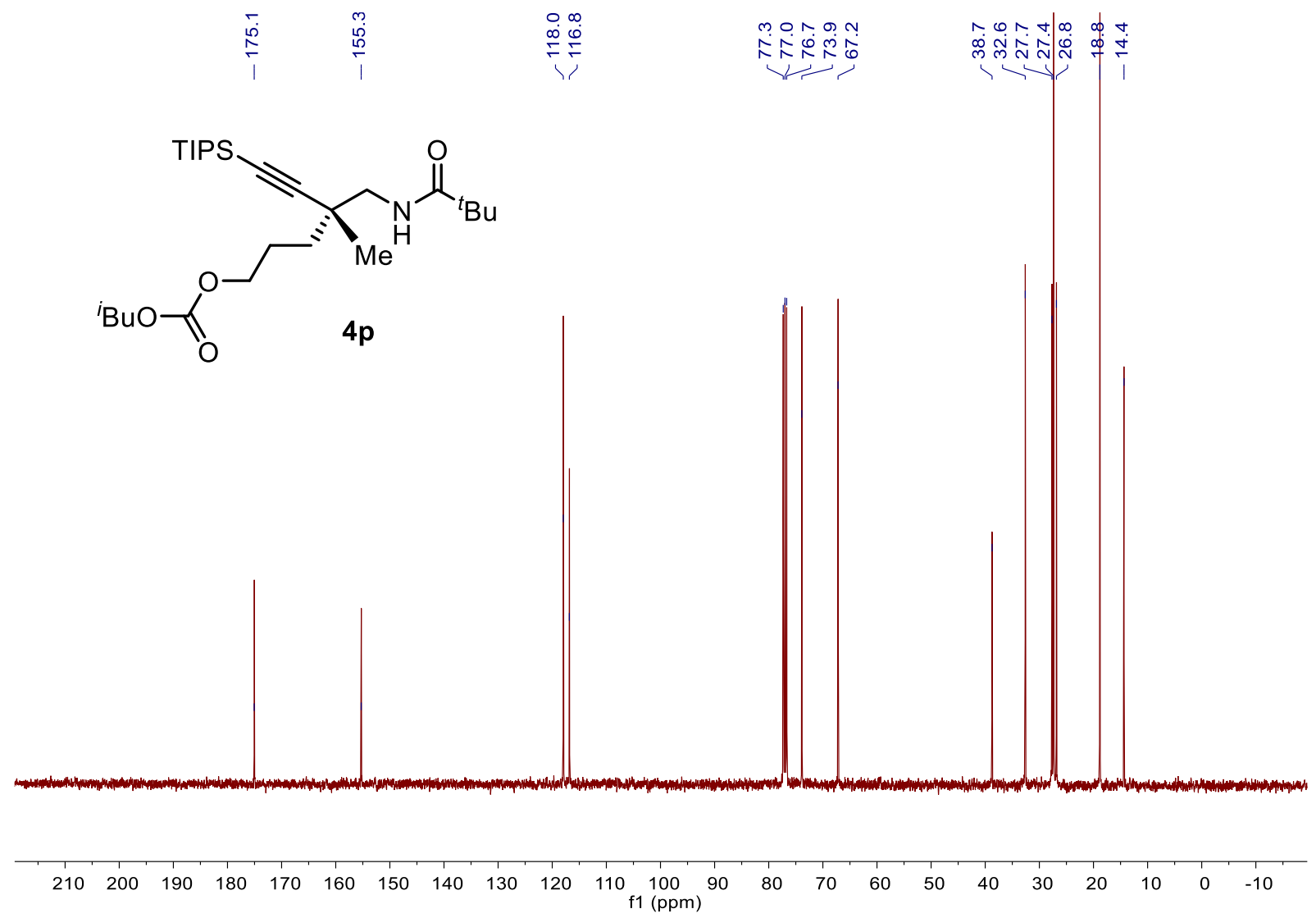

Figure S83. ${ }^{13} \mathbf{C}$ NMR $\left(101 \mathrm{MHz}, \mathrm{CDCl}_{3}\right)$ of $\mathbf{4 p}$ 


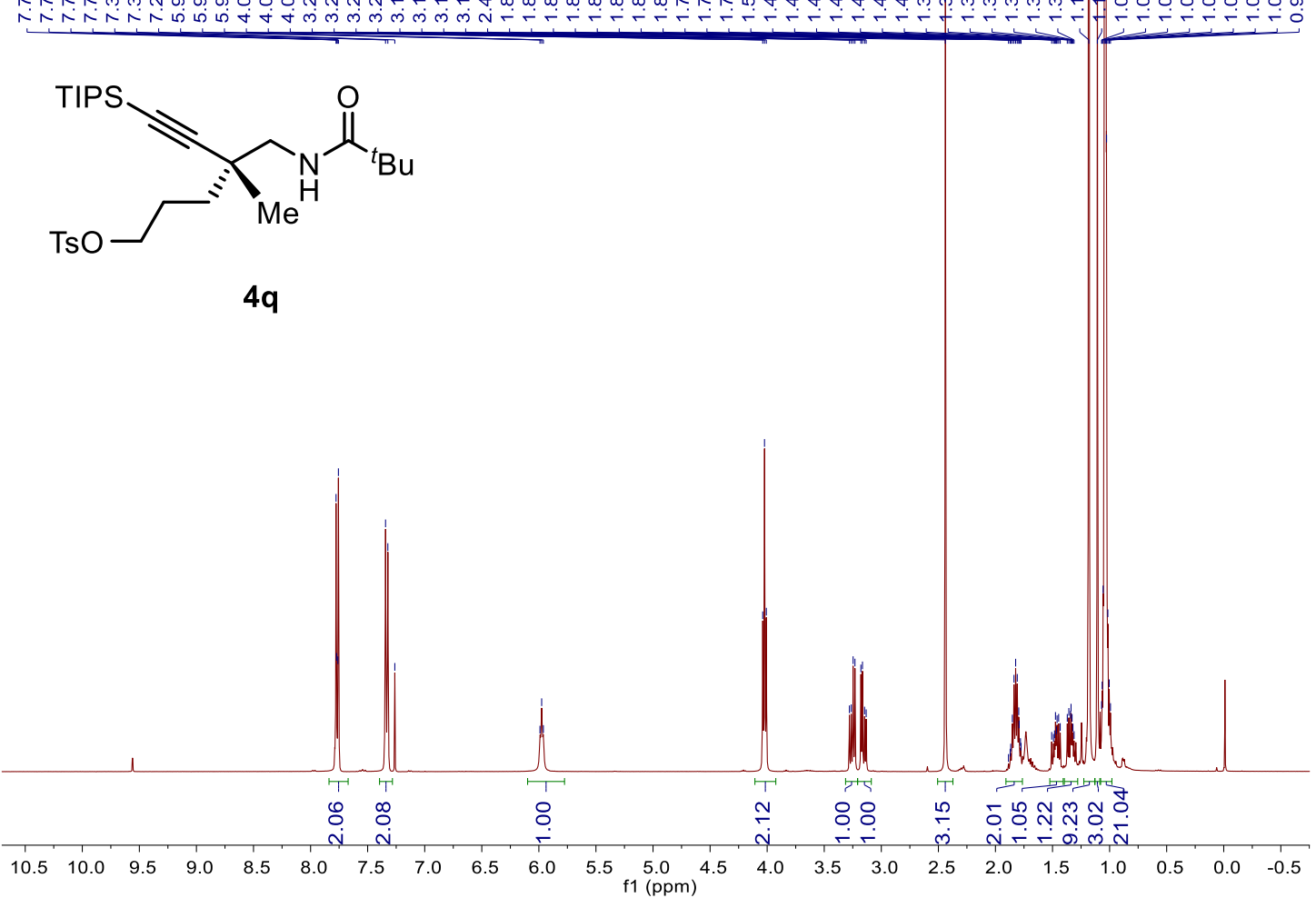

Figure S84. ${ }^{1} \mathbf{H}$ NMR $\left(400 \mathrm{MHz}, \mathrm{CDCl}_{3}\right)$ of $\mathbf{4 q}$

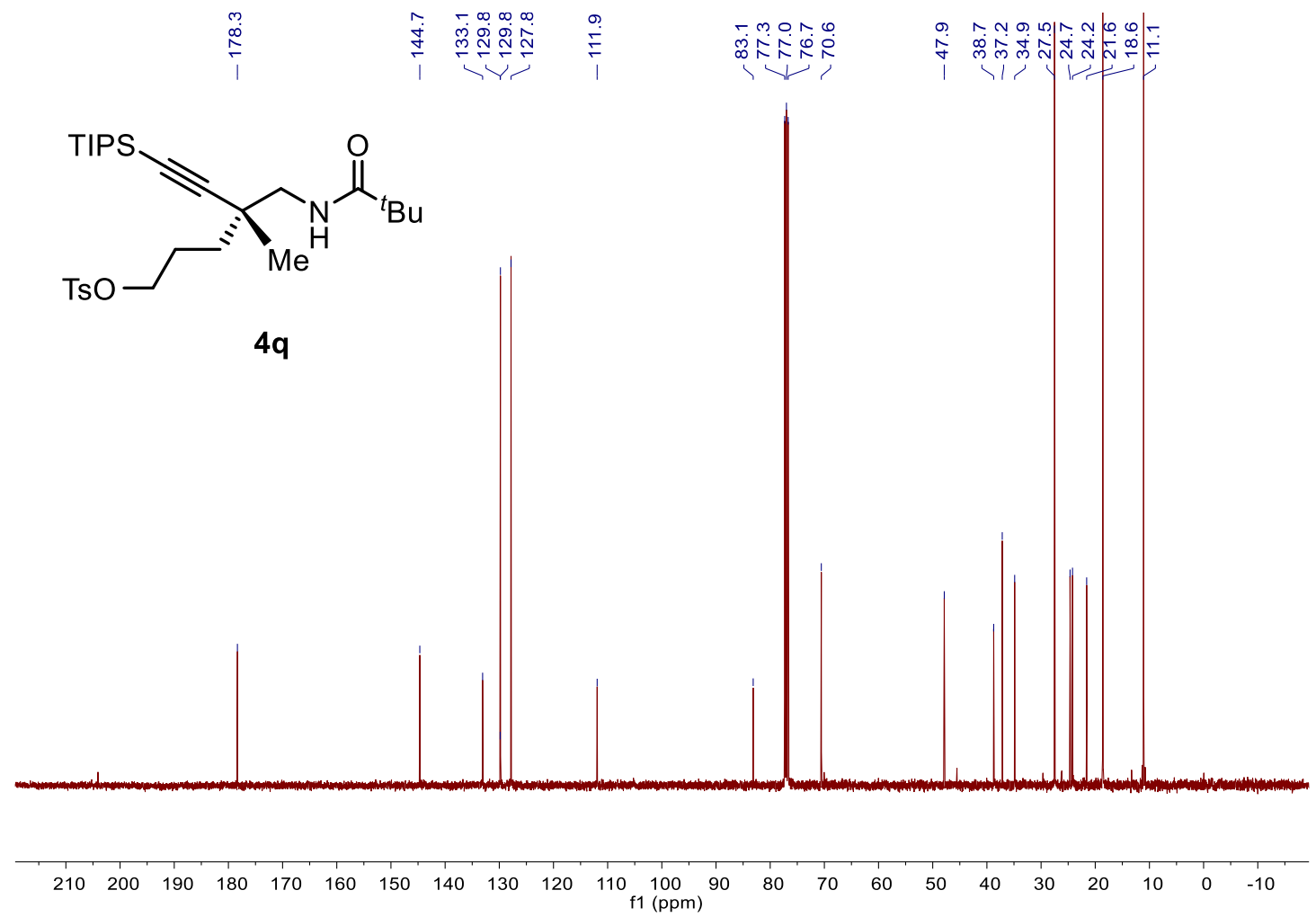

Figure S85. ${ }^{13} \mathbf{C}$ NMR $\left(101 \mathrm{MHz}, \mathrm{CDCl}_{3}\right)$ of $\mathbf{4 q}$ 


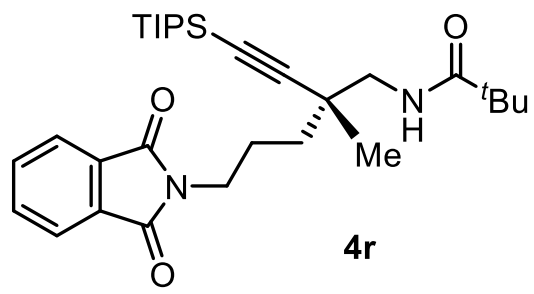

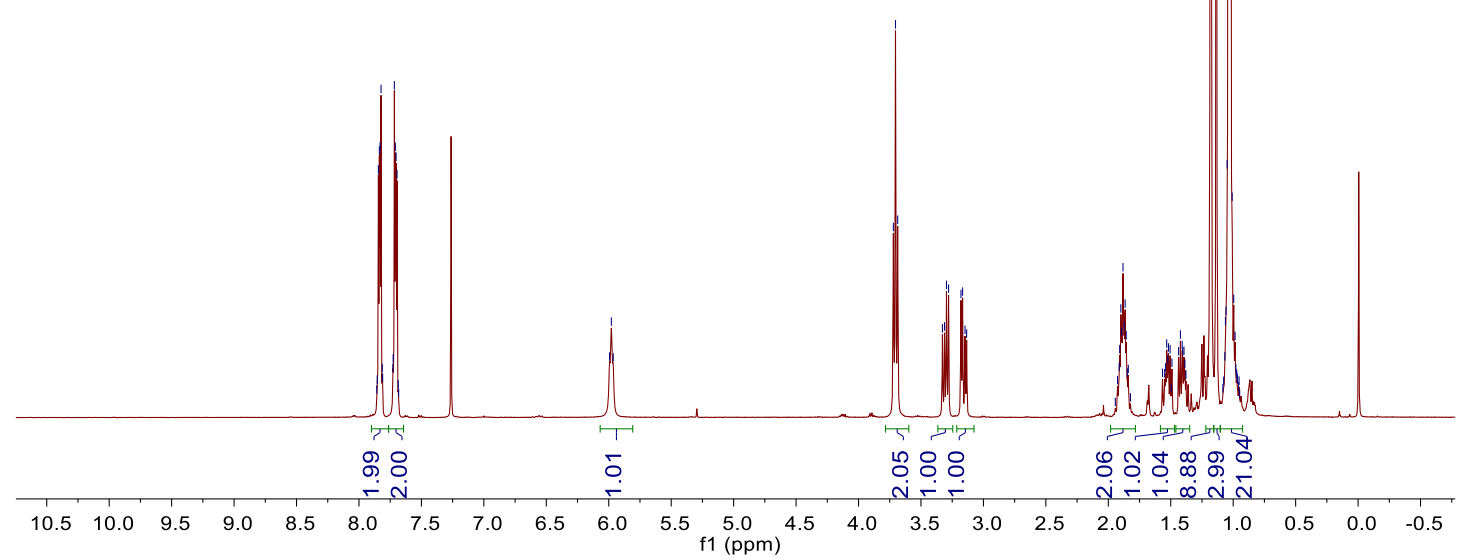

Figure S86. ${ }^{1} \mathbf{H}$ NMR $\left(400 \mathrm{MHz}, \mathrm{CDCl}_{3}\right)$ of $\mathbf{4 r}$

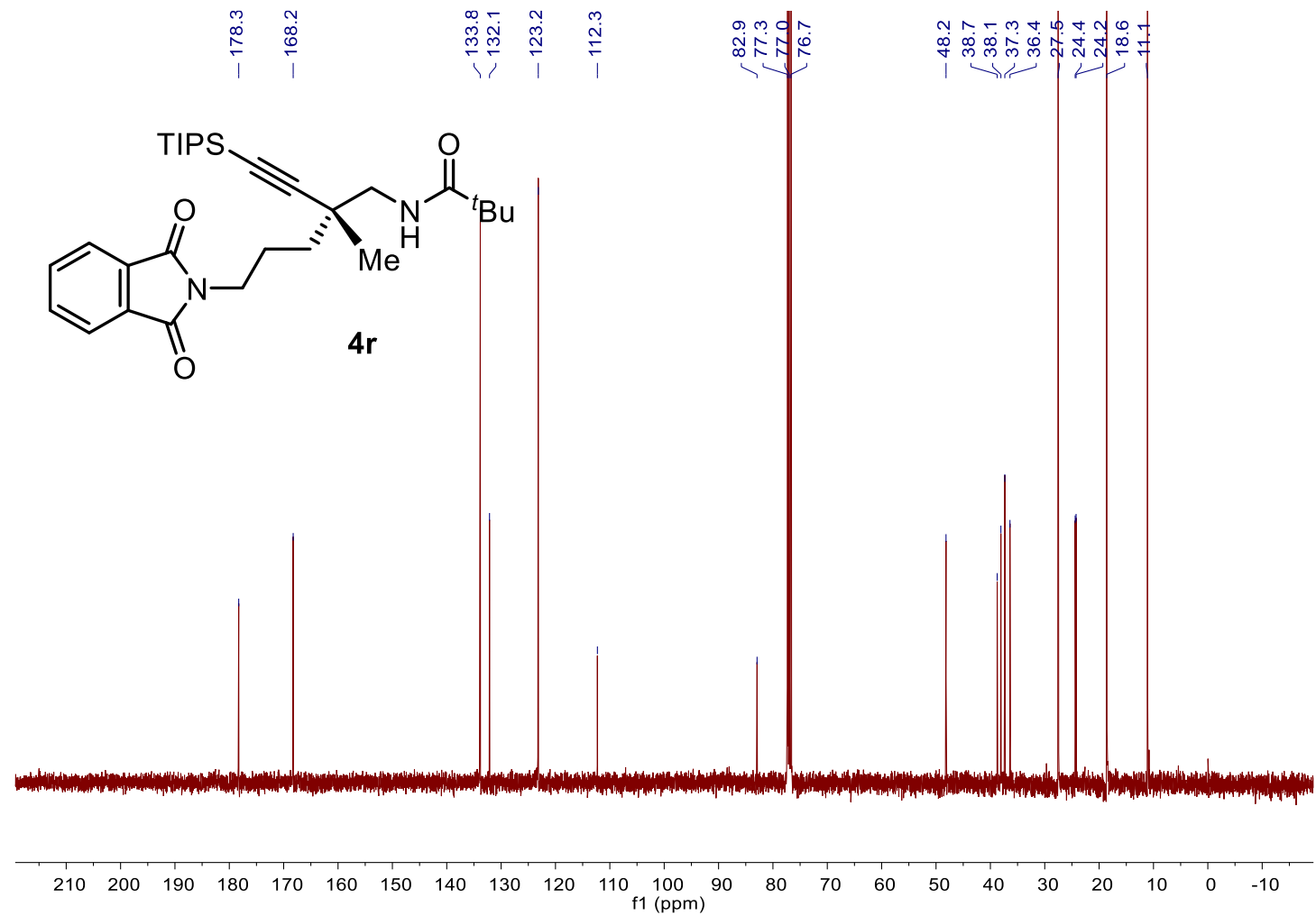

Figure S87. ${ }^{13} \mathbf{C}$ NMR $\left(101 \mathrm{MHz}, \mathrm{CDCl}_{3}\right)$ of $\mathbf{4 r}$ 


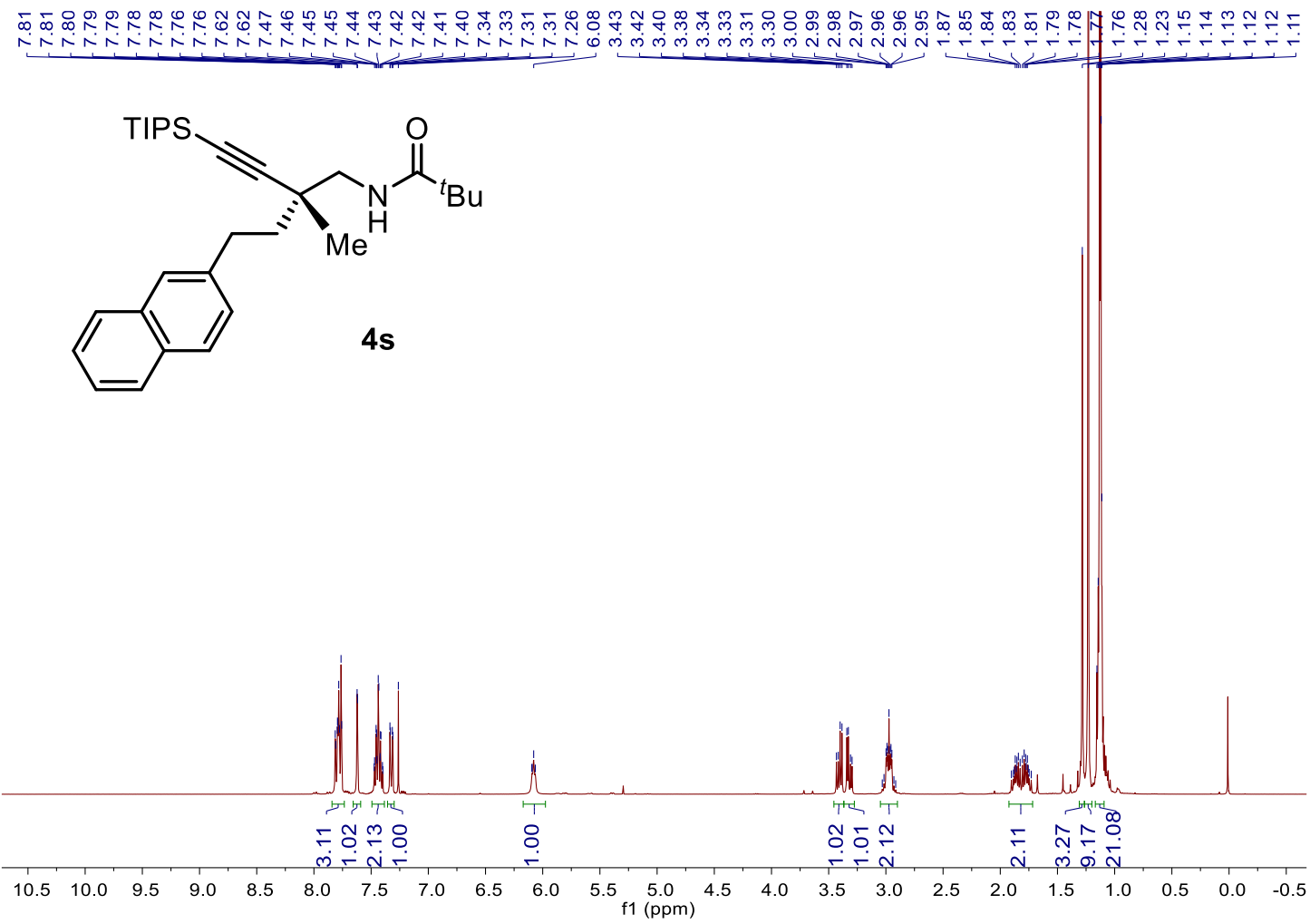

Figure S88. ${ }^{1} \mathbf{H}$ NMR $\left(400 \mathrm{MHz}, \mathrm{CDCl}_{3}\right)$ of $\mathbf{4 s}$

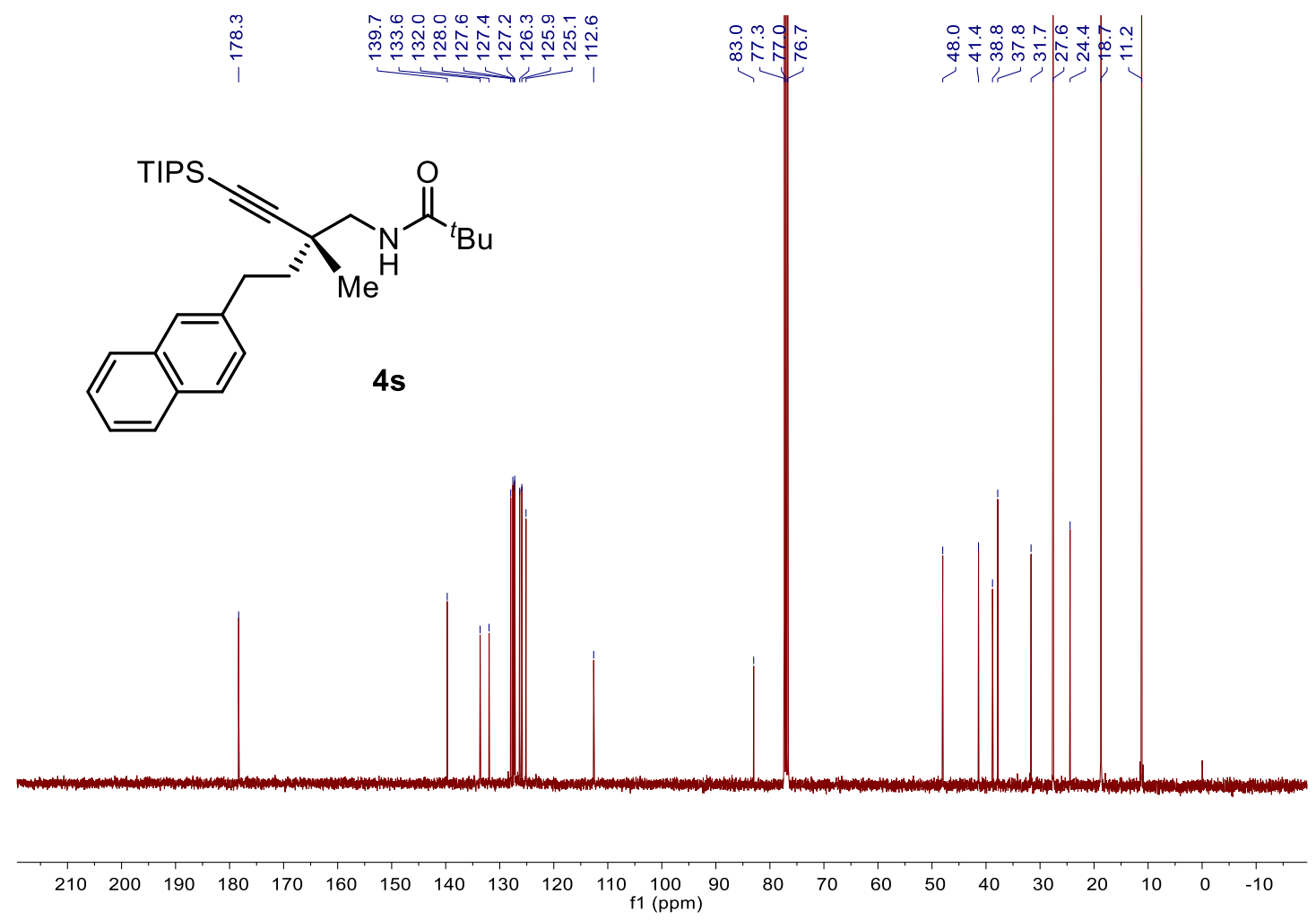

Figure S89. ${ }^{13} \mathbf{C}$ NMR $\left(101 \mathrm{MHz}, \mathrm{CDCl}_{3}\right)$ of $\mathbf{4 s}$ 


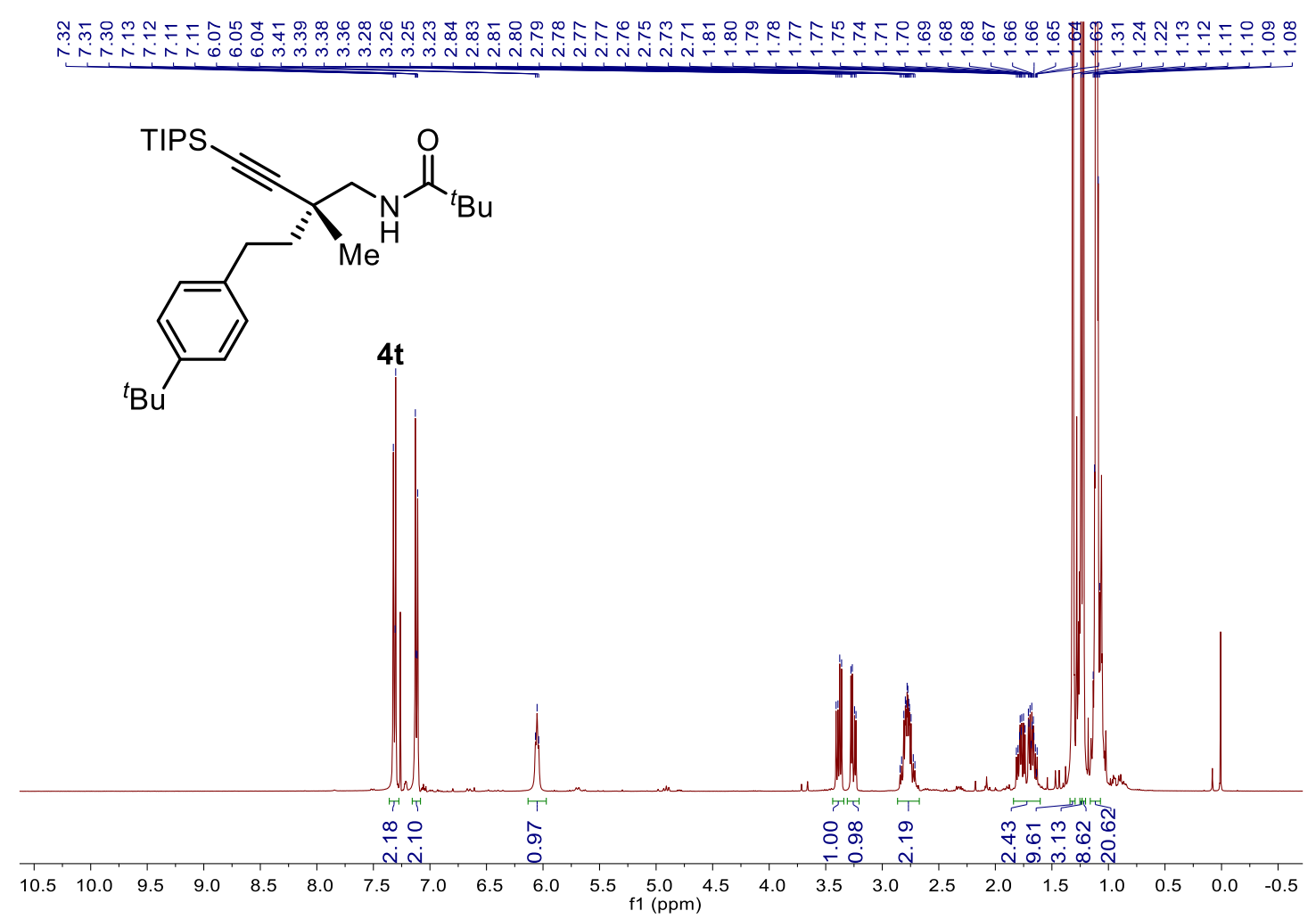

Figure S90. ${ }^{\mathbf{1}} \mathbf{H}$ NMR $\left(400 \mathrm{MHz}, \mathrm{CDCl}_{3}\right)$ of $\mathbf{4 t}$

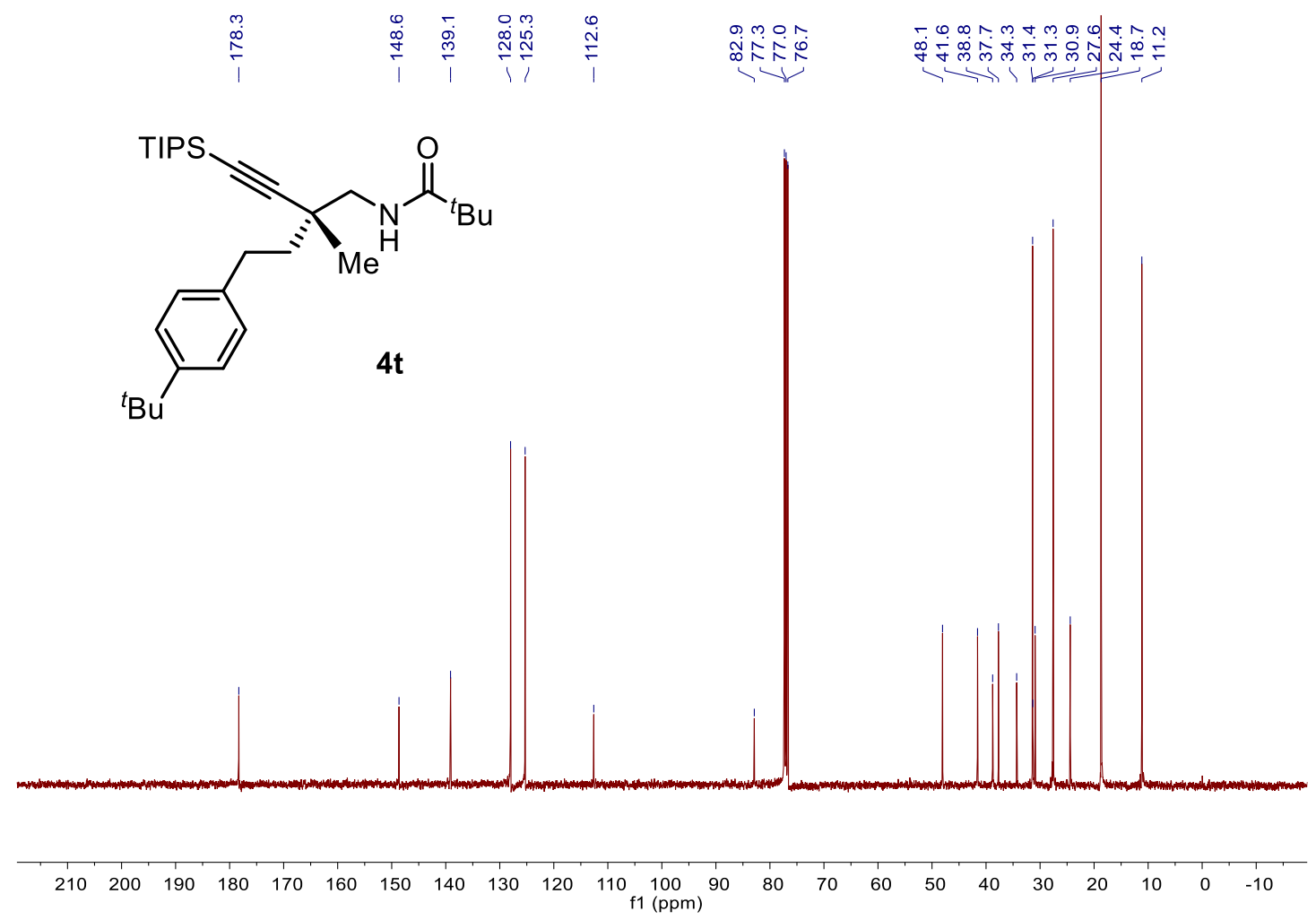

Figure S91. ${ }^{13} \mathbf{C}$ NMR $\left(101 \mathrm{MHz}, \mathrm{CDCl}_{3}\right)$ of $\mathbf{4 t}$ 


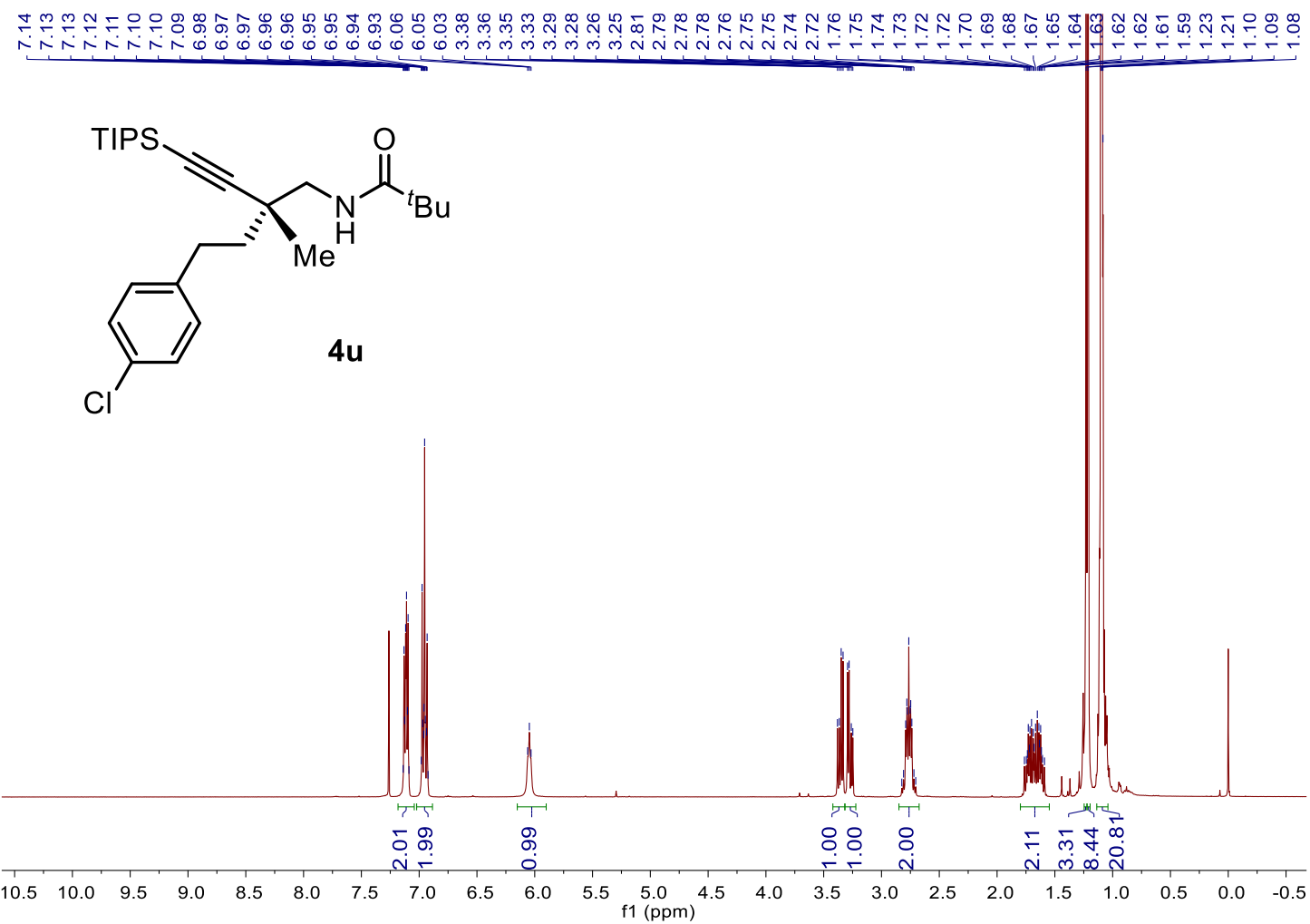

Figure S92. ${ }^{1} \mathbf{H}$ NMR $\left(400 \mathrm{MHz}, \mathrm{CDCl}_{3}\right)$ of $\mathbf{4} \mathbf{u}$

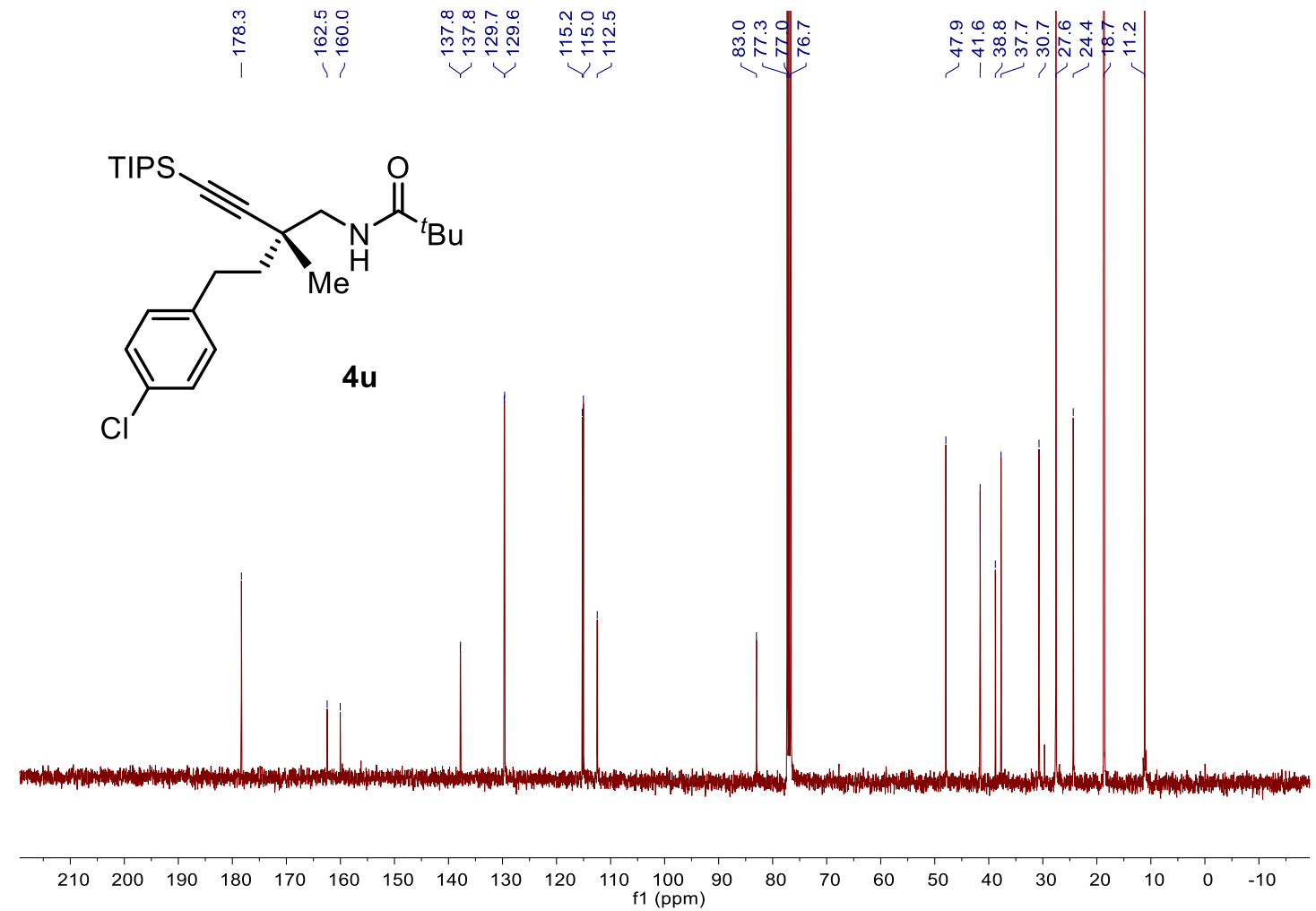

Figure S93. ${ }^{13} \mathbf{C}$ NMR $\left(101 \mathrm{MHz}, \mathrm{CDCl}_{3}\right)$ of $\mathbf{4 u}$ 


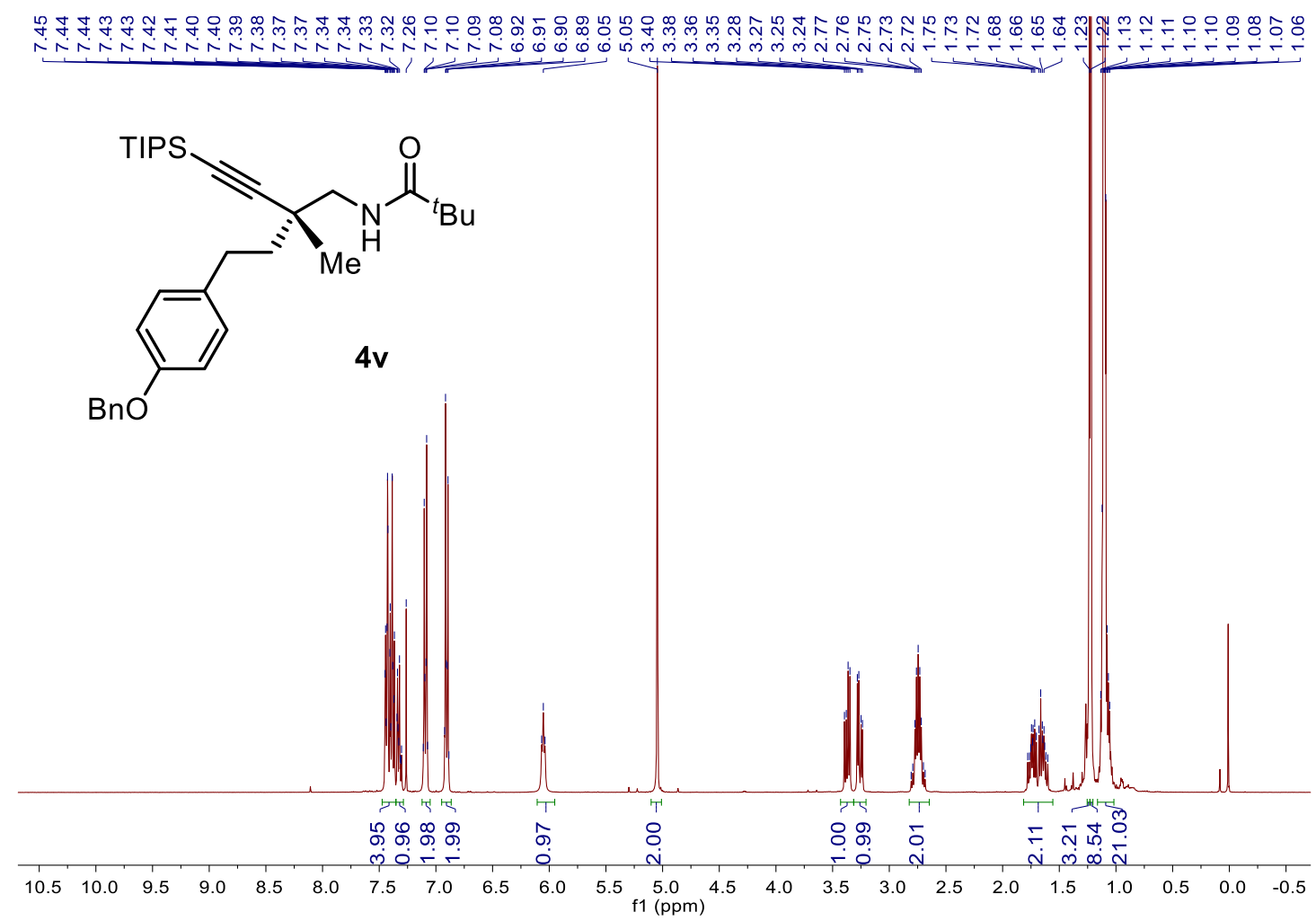

Figure S94. ${ }^{1} \mathbf{H}$ NMR $\left(400 \mathrm{MHz}, \mathrm{CDCl}_{3}\right)$ of $\mathbf{4 v}$

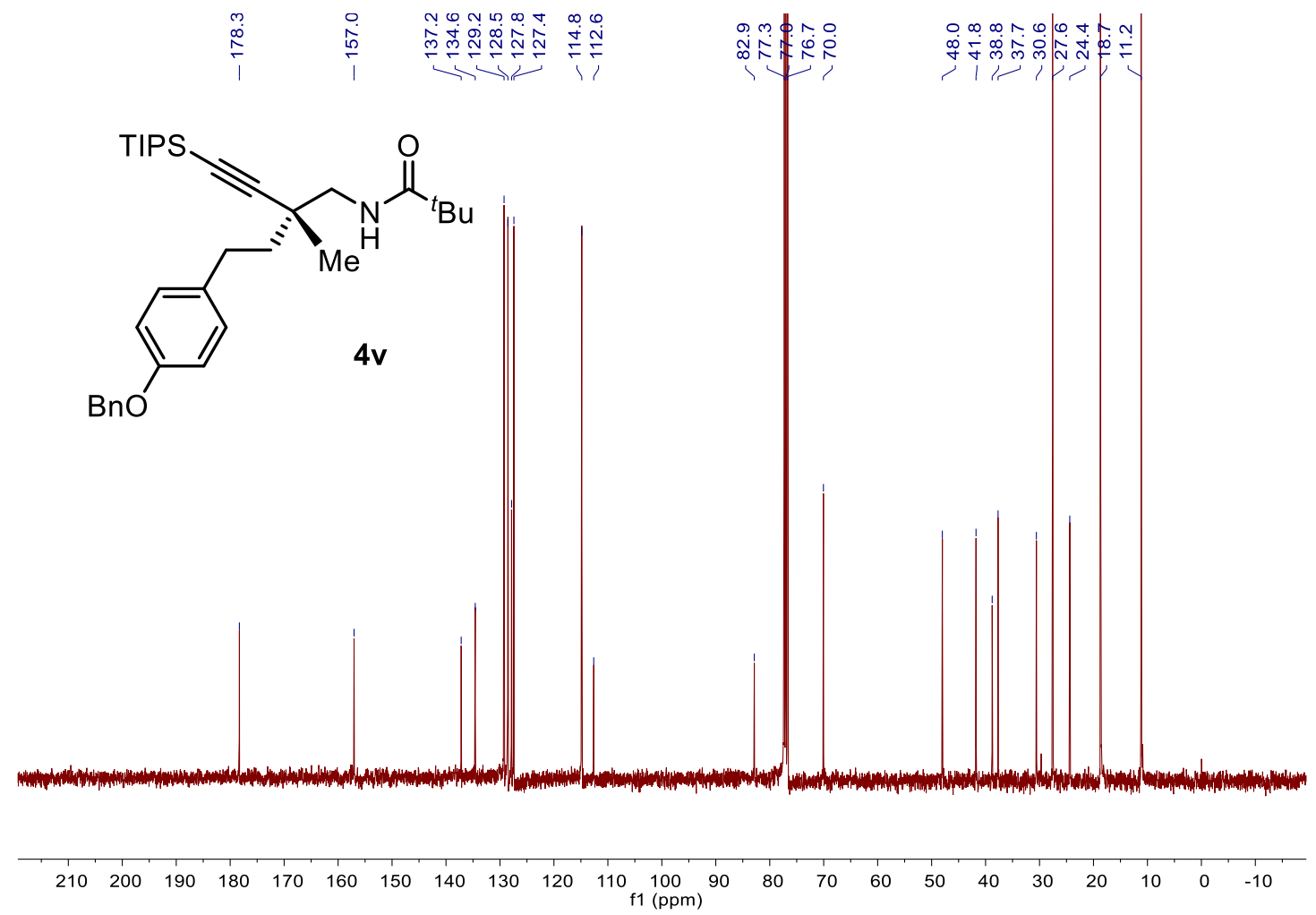

Figure S95. ${ }^{13} \mathbf{C}$ NMR $\left(101 \mathrm{MHz}, \mathrm{CDCl}_{3}\right)$ of $\mathbf{4 v}$ 


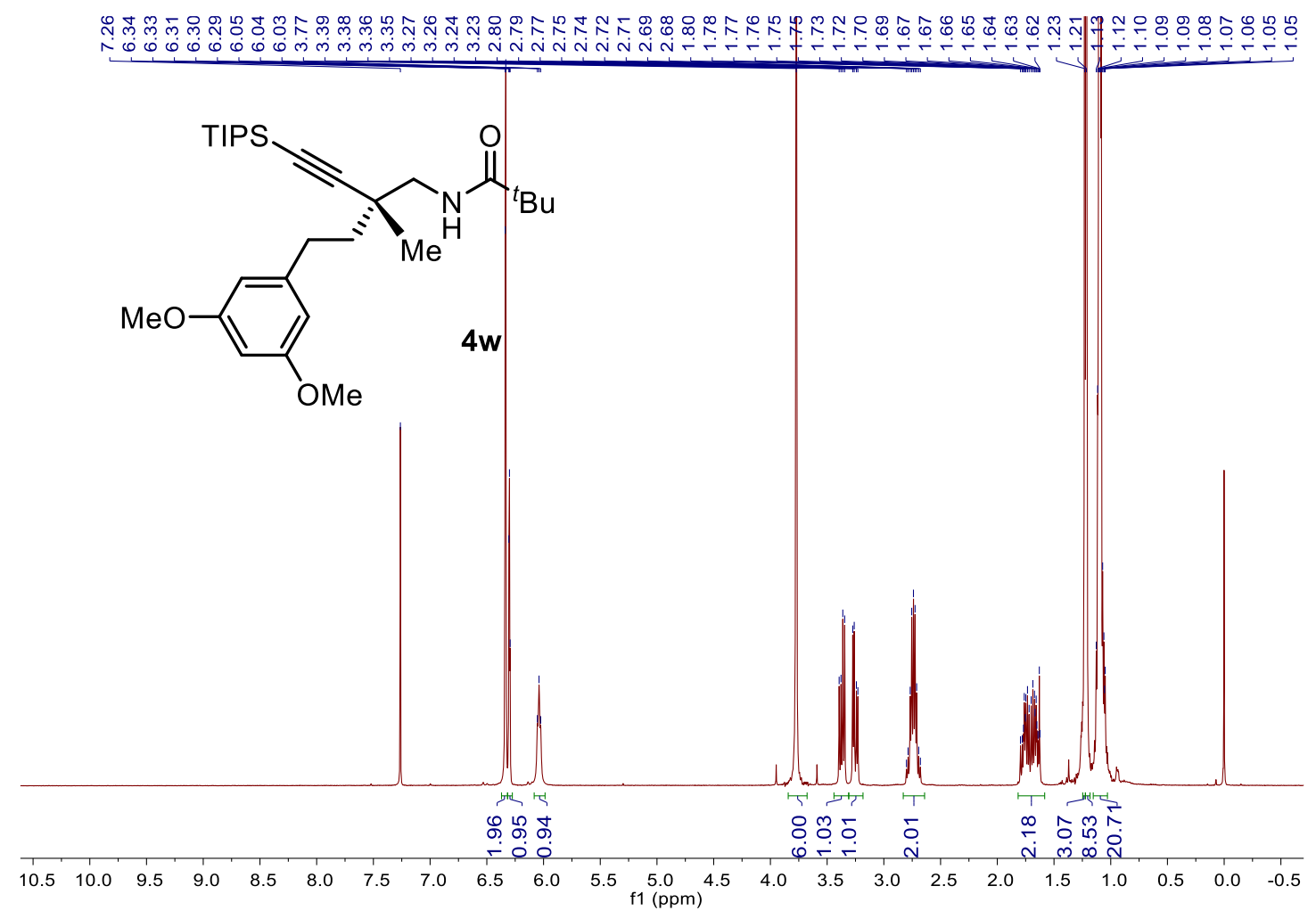

Figure S96. ${ }^{1} \mathbf{H}$ NMR $\left(400 \mathrm{MHz}, \mathrm{CDCl}_{3}\right)$ of $\mathbf{4 w}$

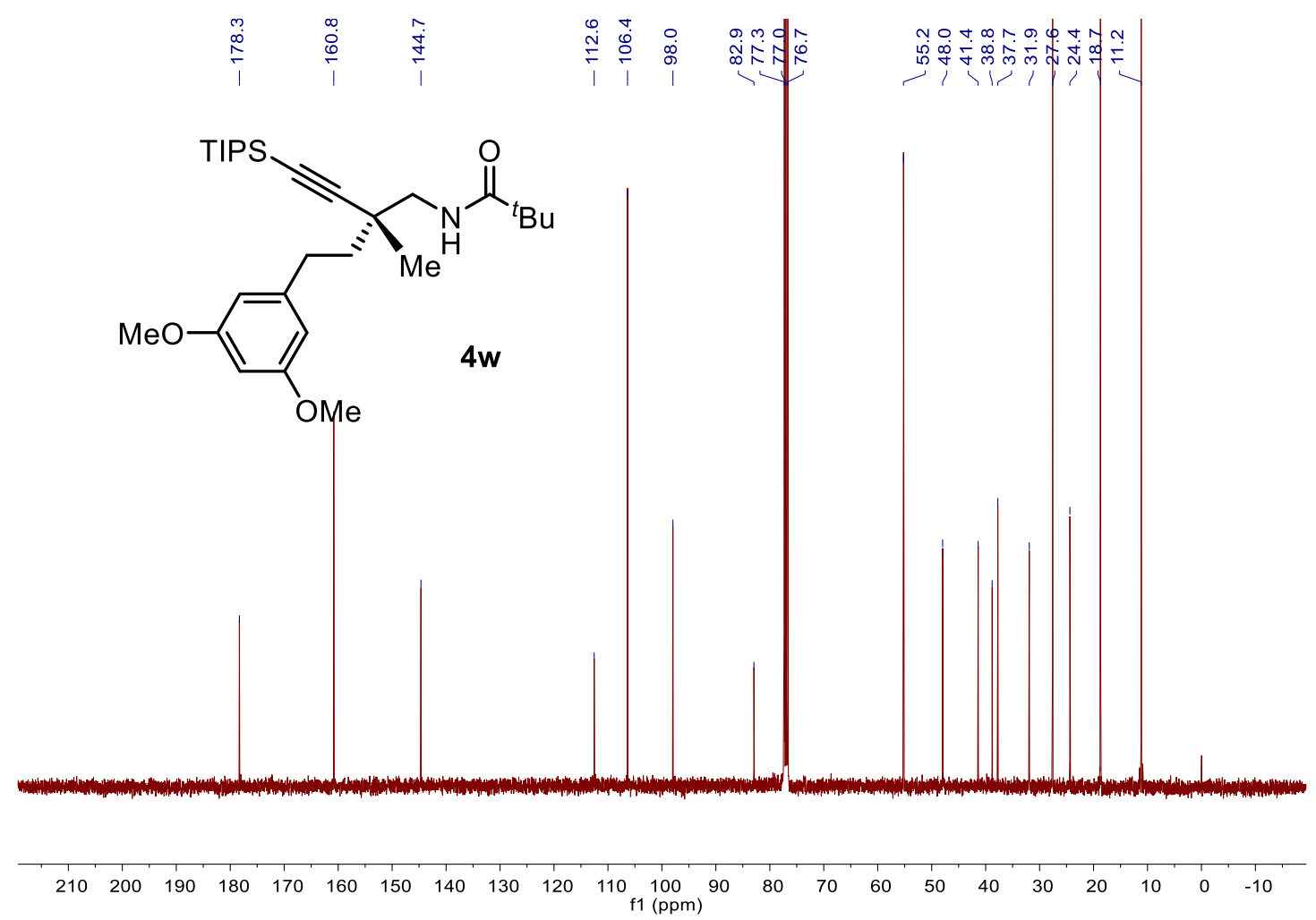

Figure S97. ${ }^{13} \mathbf{C}$ NMR $\left(101 \mathrm{MHz}, \mathrm{CDCl}_{3}\right)$ of $\mathbf{4 w}$ 


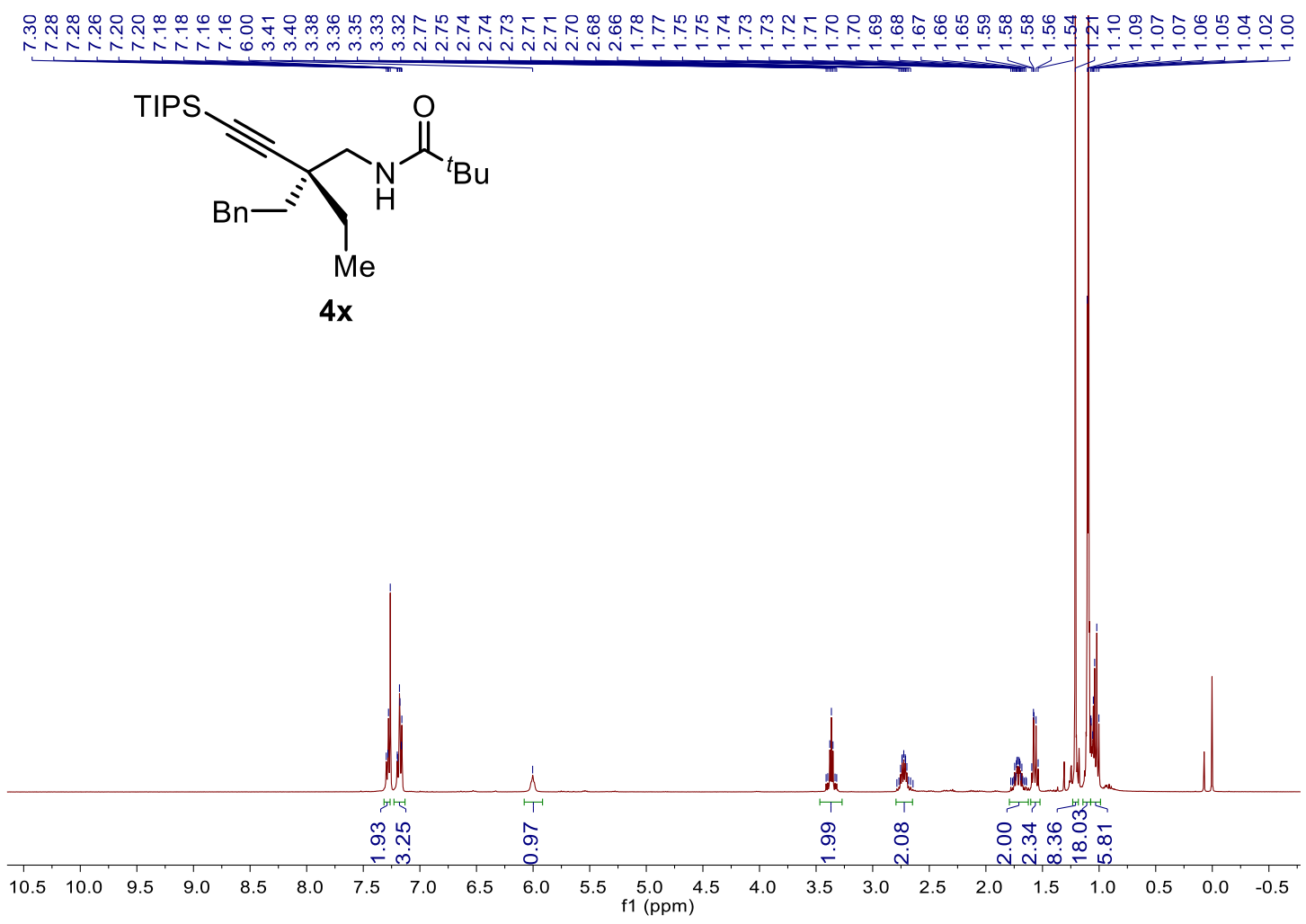

Figure S98. ${ }^{1} \mathbf{H}$ NMR $\left(400 \mathrm{MHz}, \mathrm{CDCl}_{3}\right)$ of $\mathbf{4 x}$

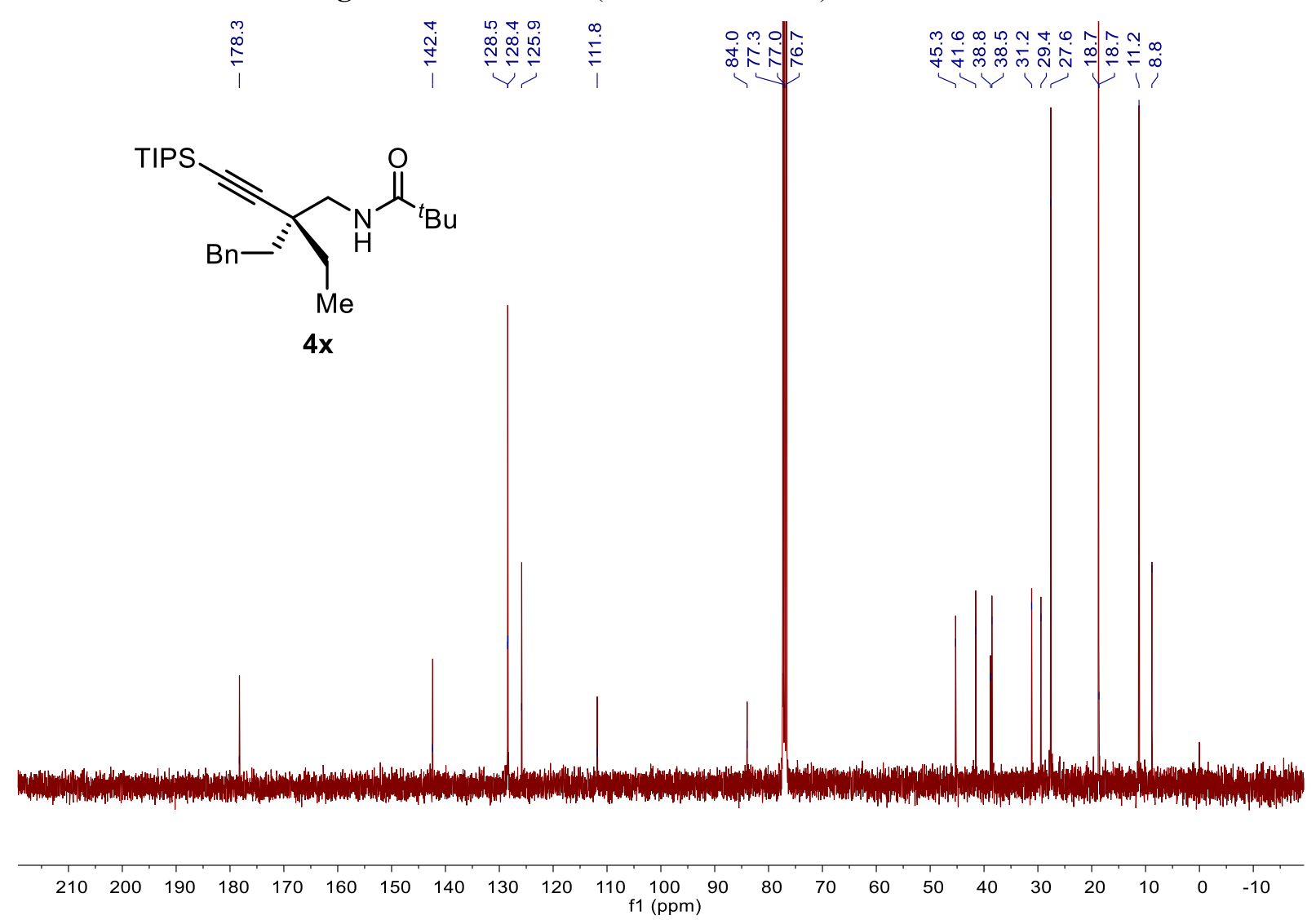

Figure S99. ${ }^{13} \mathbf{C} \mathbf{~ N M R}\left(101 \mathrm{MHz}, \mathrm{CDCl}_{3}\right)$ of $\mathbf{4} \mathbf{x}$

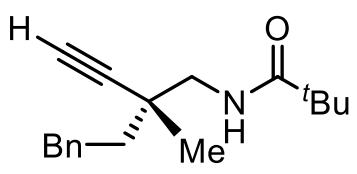




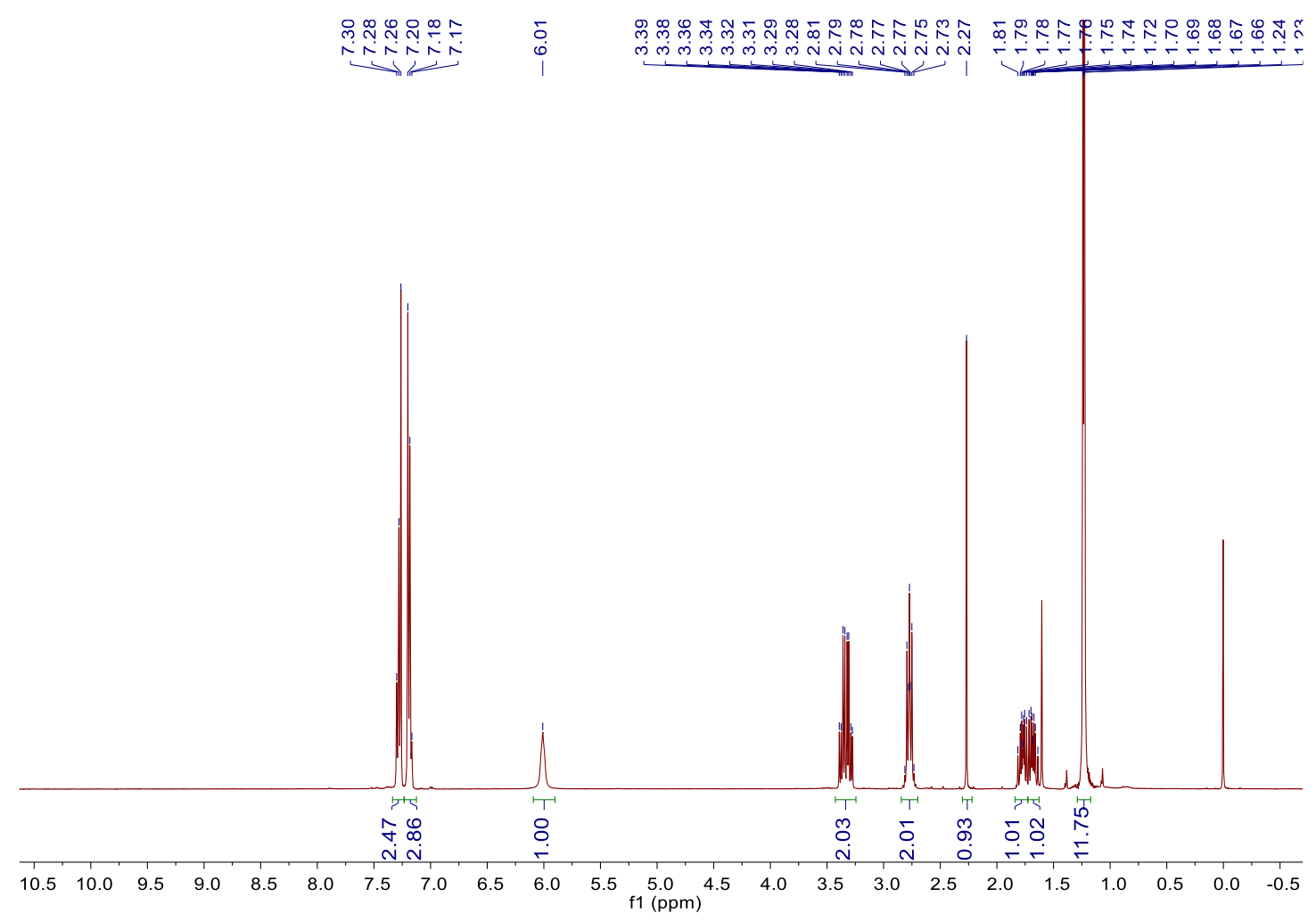

Figure S100. ${ }^{1} \mathbf{H}$ NMR $\left(400 \mathrm{MHz}, \mathrm{CDCl}_{3}\right)$ of $\mathbf{5 a}$

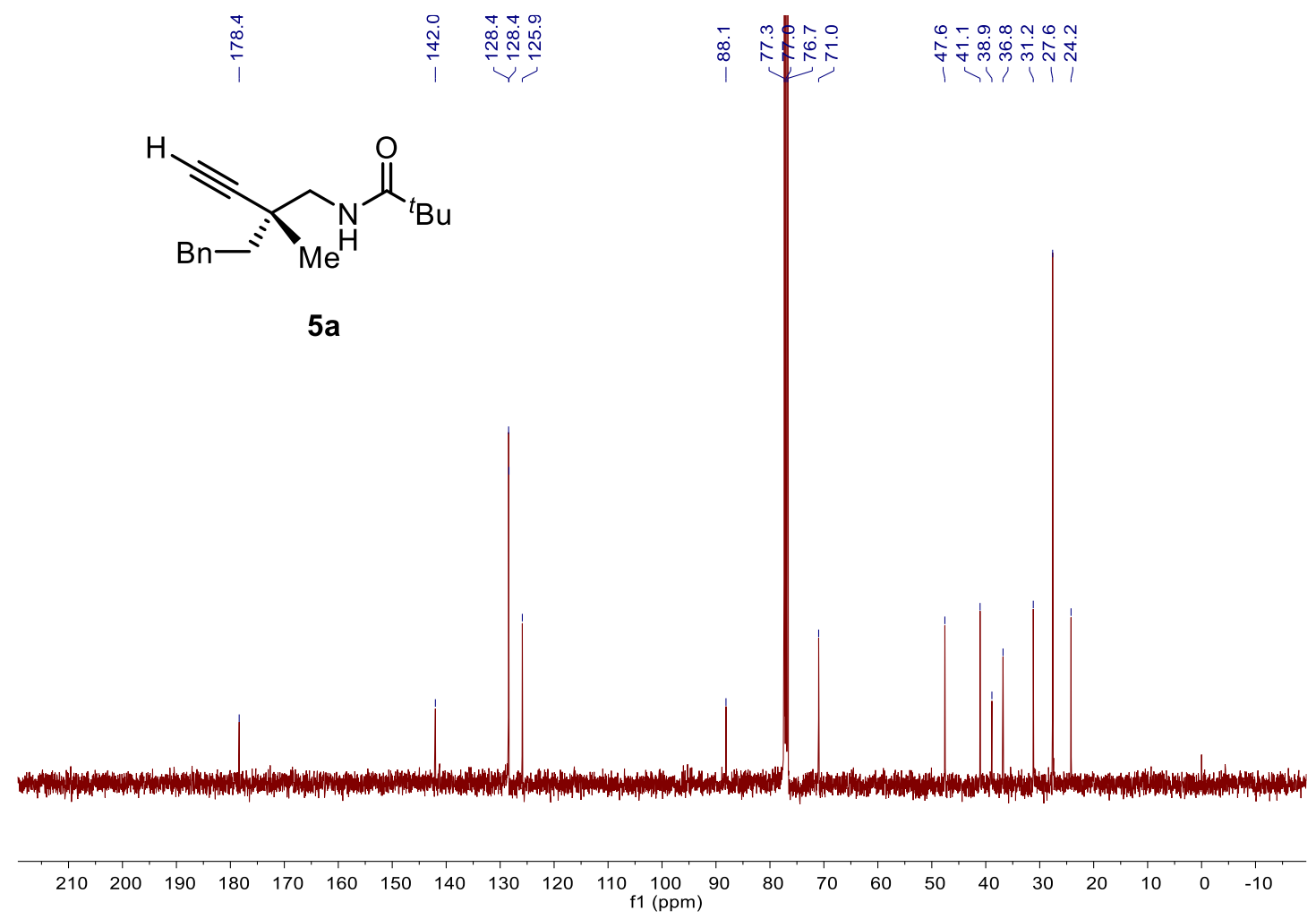

Figure S101. ${ }^{13} \mathbf{C}$ NMR $\left(101 \mathrm{MHz}, \mathrm{CDCl}_{3}\right)$ of $5 \mathbf{a}$ 


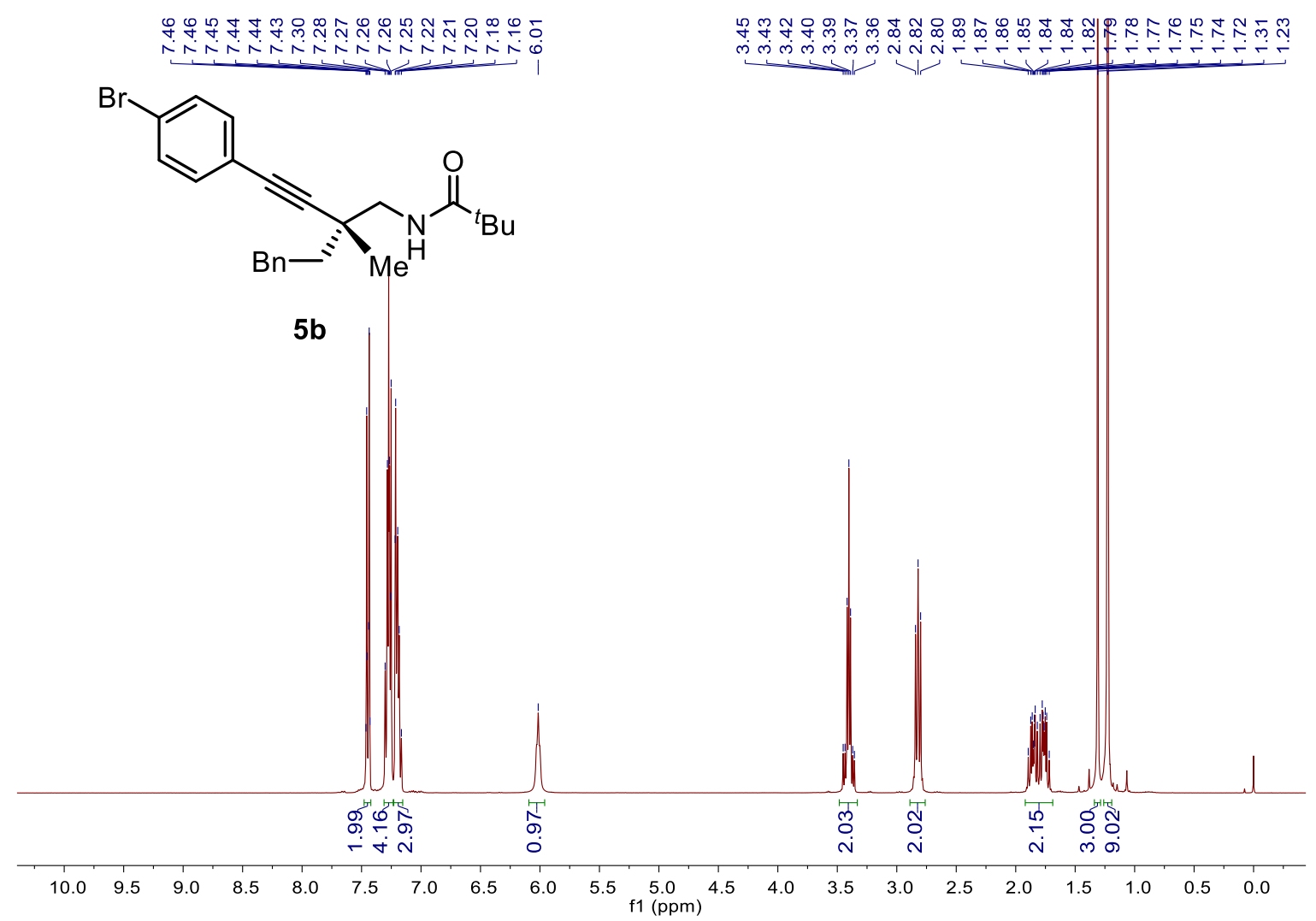

Figure S102. ${ }^{1} \mathbf{H}$ NMR $\left(400 \mathrm{MHz}, \mathrm{CDCl}_{3}\right)$ of $\mathbf{5 b}$

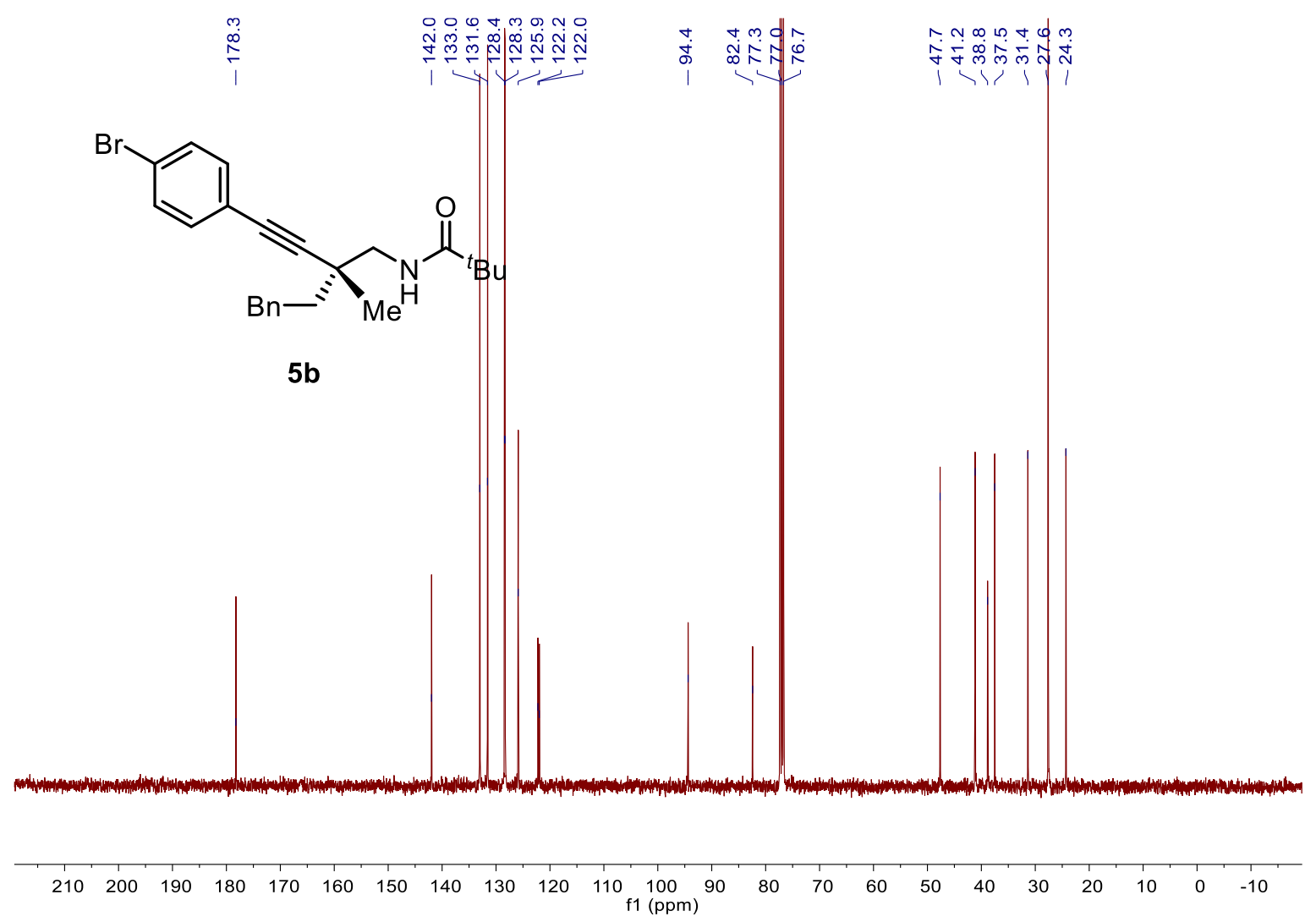

Figure S103. ${ }^{13} \mathbf{C}$ NMR $\left(101 \mathrm{MHz}, \mathrm{CDCl}_{3}\right)$ of $\mathbf{5 b}$ 
<smiles>C=CC(CBr)(CBr)CNC(=O)CBr</smiles>

$5 c$

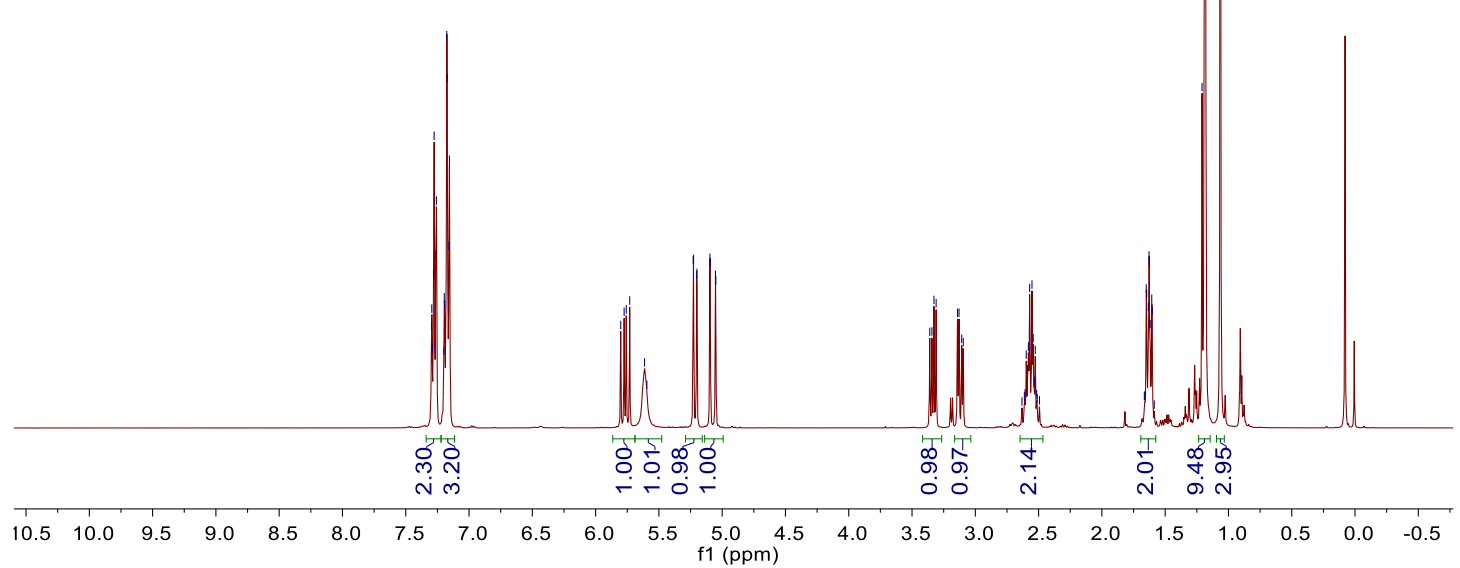

Figure S104. ${ }^{1} \mathbf{H}$ NMR $\left(400 \mathrm{MHz}, \mathrm{CDCl}_{3}\right)$ of $\mathbf{5 c}$<smiles>C=CC(CBr)(CBr)CNC(=O)CBr</smiles>

$5 c$

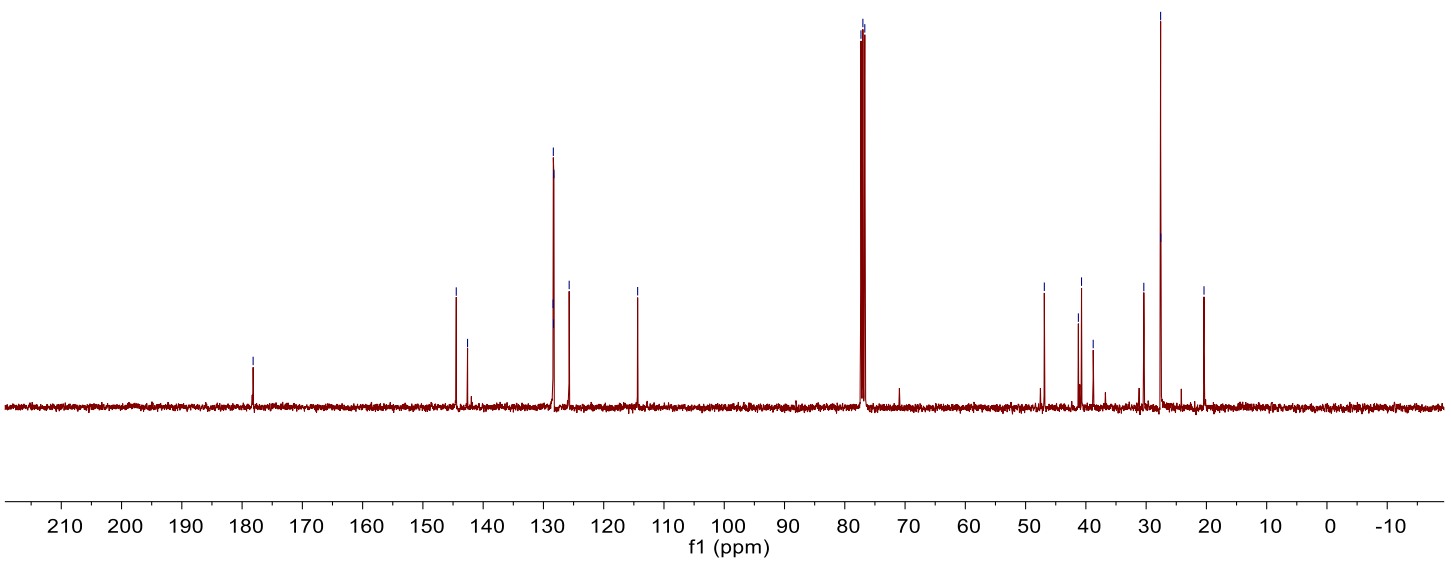

Figure S105. ${ }^{13} \mathbf{C}$ NMR $\left(101 \mathrm{MHz}, \mathrm{CDCl}_{3}\right)$ of $\mathbf{5 c}$ 


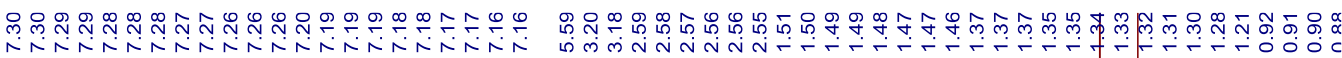<smiles>CCC(C)(CNC(=O)CBr)C(=O)OC</smiles>

5d

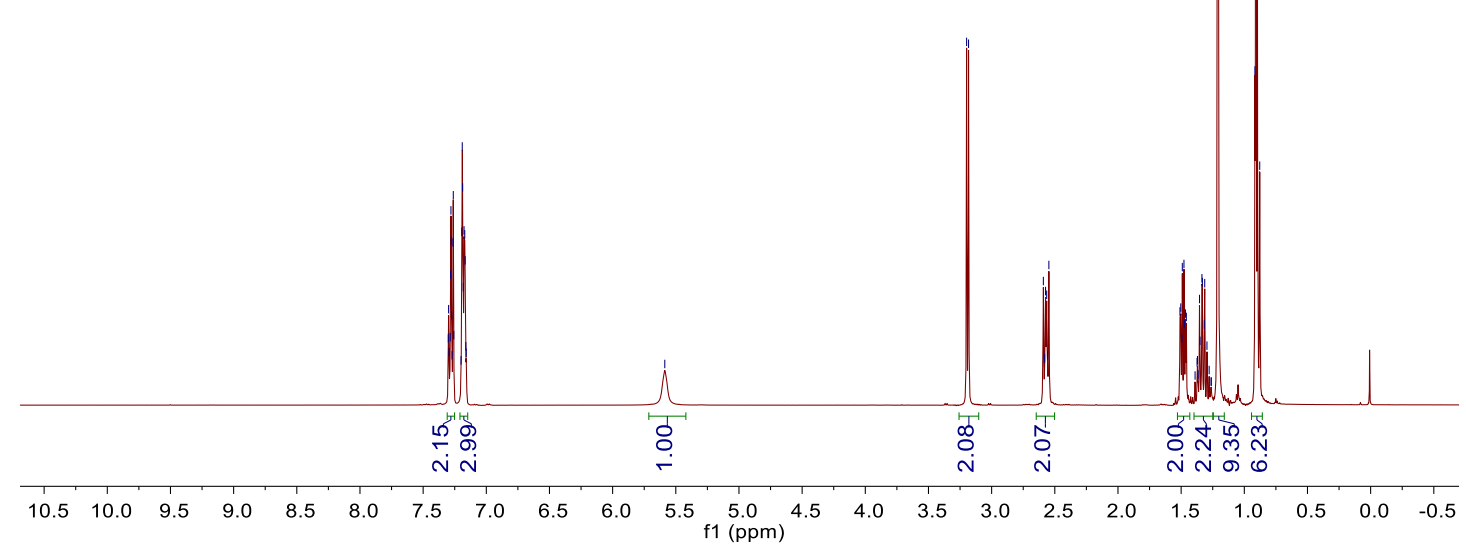

Figure S106. ${ }^{1} \mathbf{H}$ NMR $\left(400 \mathrm{MHz}, \mathrm{CDCl}_{3}\right)$ of $\mathbf{5 d}$

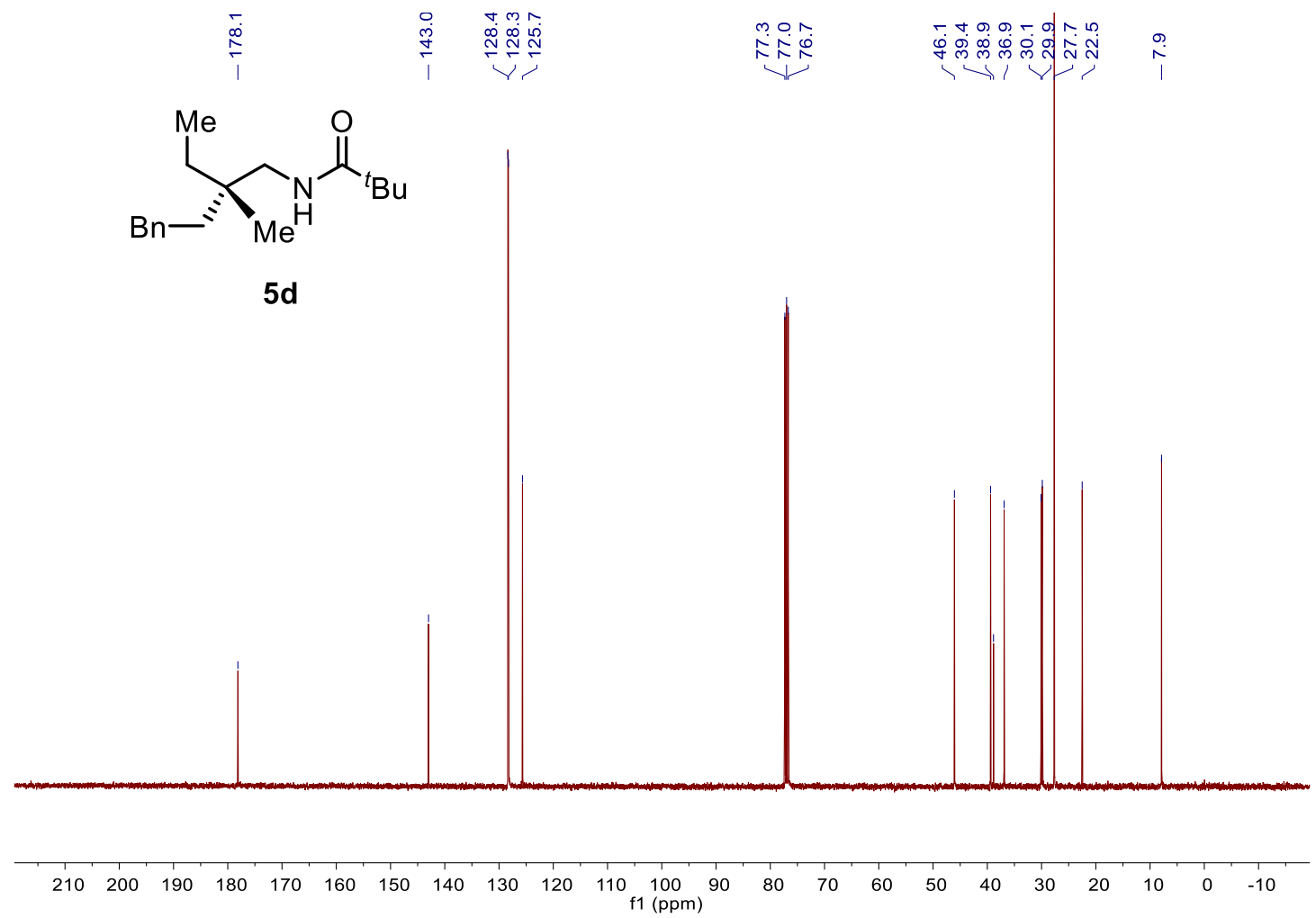

Figure S107. ${ }^{13} \mathbf{C}$ NMR $\left(101 \mathrm{MHz}, \mathrm{CDCl}_{3}\right)$ of $\mathbf{5 d}$ 
<smiles>CC(C)(C)C(=O)NC[C@](C)(CBr)C(=O)O</smiles>

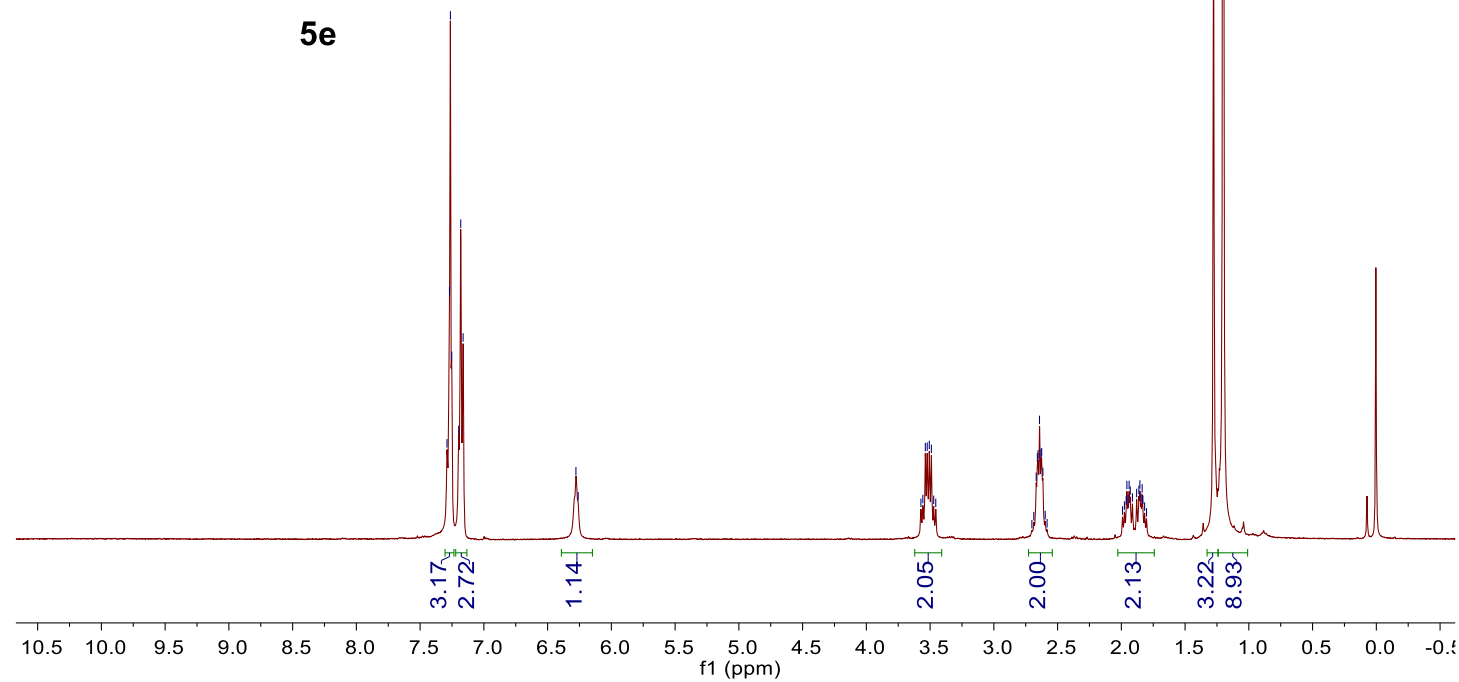

Figure S108. ${ }^{1} \mathbf{H}$ NMR $\left(400 \mathrm{MHz}, \mathrm{CDCl}_{3}\right)$ of $\mathbf{5 e}$

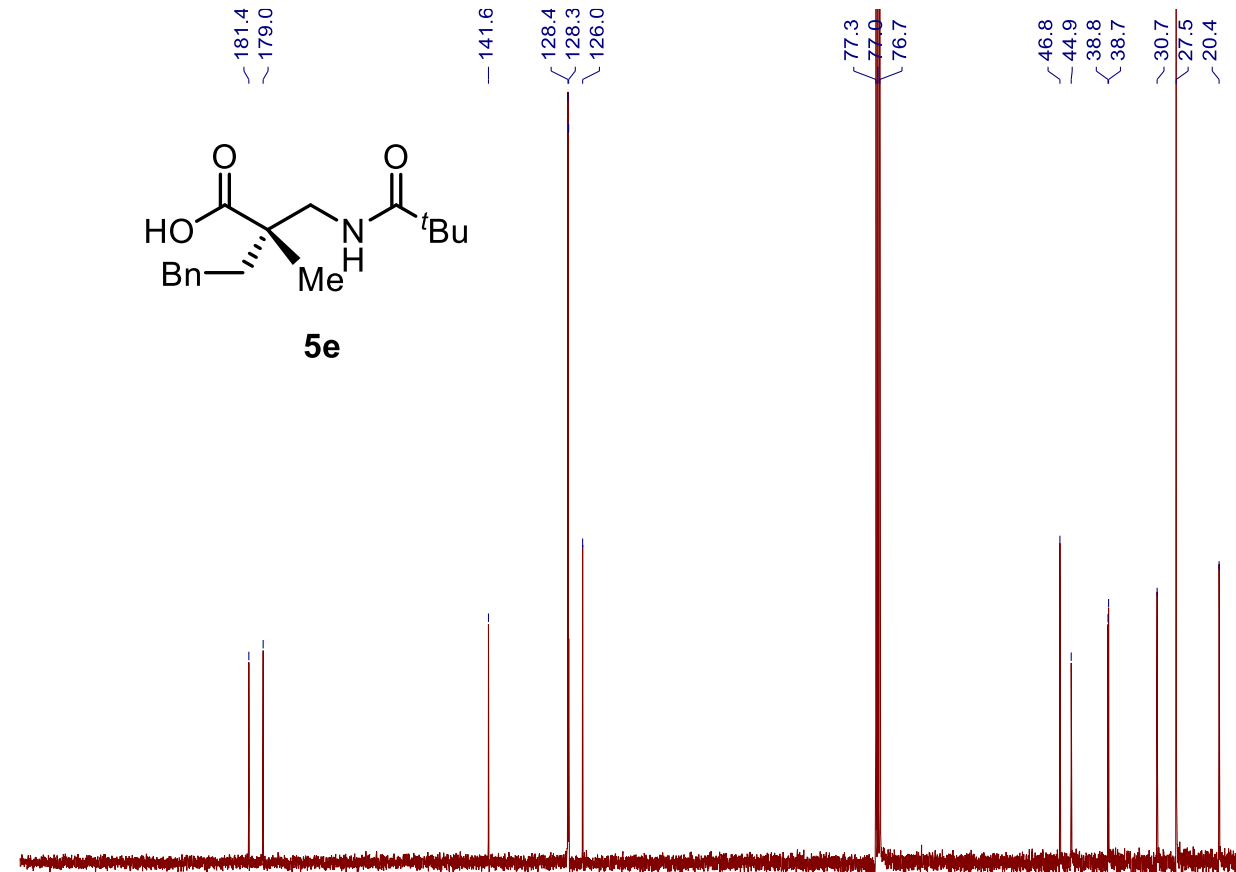

$5 e$

$\begin{array}{llllllllllll}210 & 200 & 190 & 180 & 170 & 160 & 150 & 140 & 130 & 120 & 110 \underset{\mathrm{f} 1}{100}(\mathrm{ppm})\end{array}$

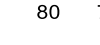

50

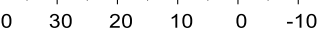

Figure S109. ${ }^{13} \mathbf{C}$ NMR $\left(101 \mathrm{MHz}, \mathrm{CDCl}_{3}\right)$ of $\mathbf{5 e}$ 
Ts<smiles>CC(C)CC(=O)NC[C@@](C)(CBr)c1cn(C)nn1</smiles>

$5 f$

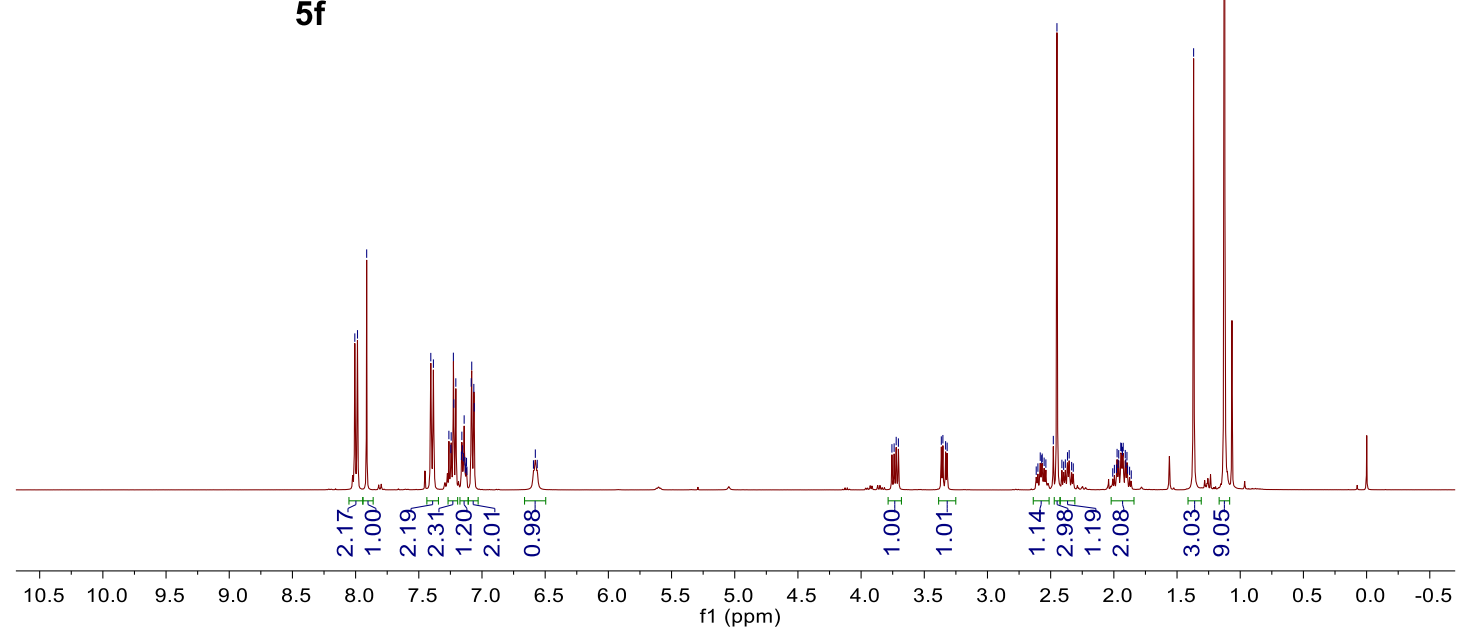

Figure S110. ${ }^{1} \mathbf{H}$ NMR $\left(400 \mathrm{MHz}, \mathrm{CDCl}_{3}\right)$ of $\mathbf{5 f}$
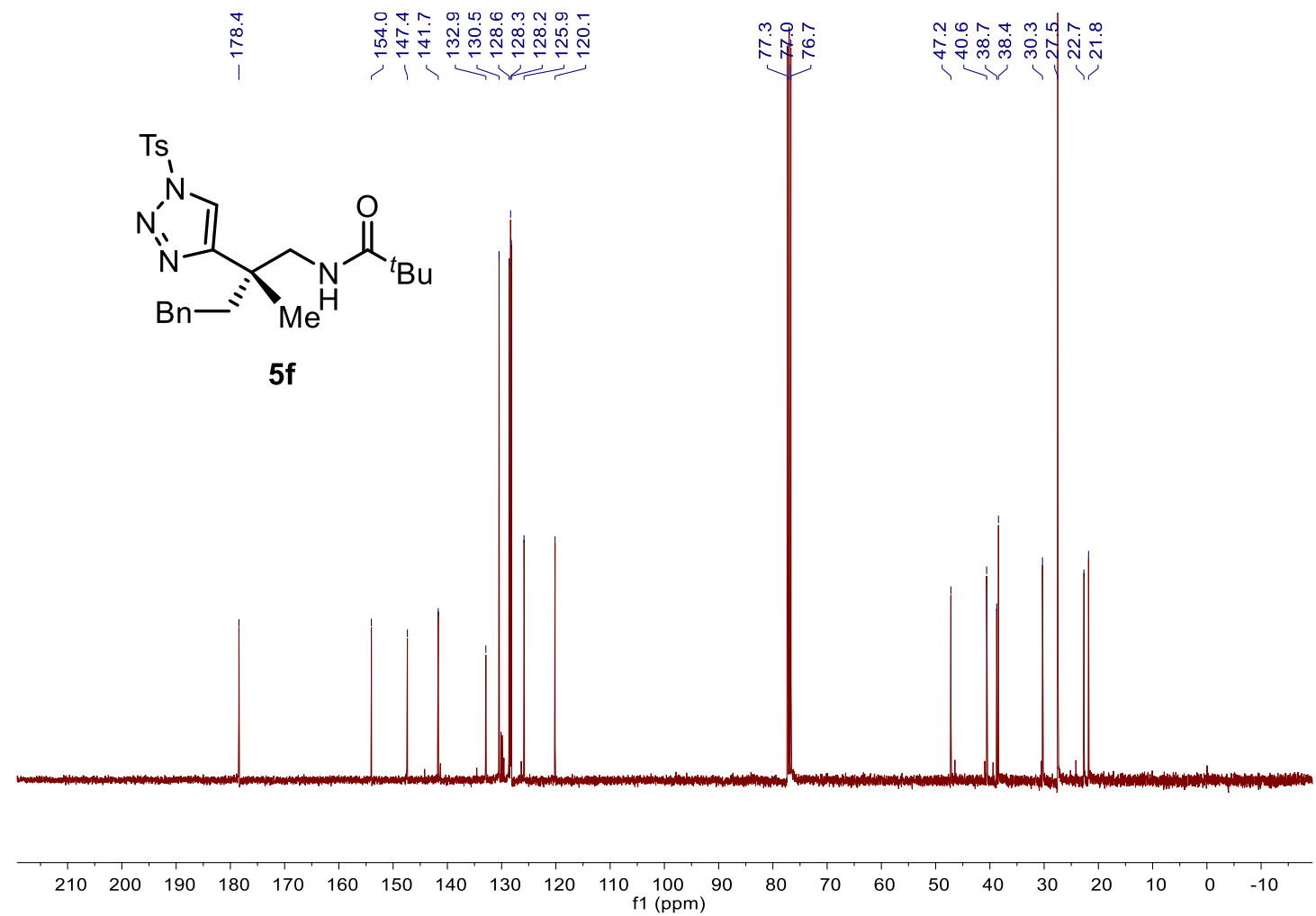

$5 f$

$\begin{array}{llllllllllll}210 & 200 & 190 & 180 & 170 & 160 & 150 & 140 & 130 & 120 & 110 \underset{\mathrm{f} 1}{100}(\mathrm{ppm}) & 90\end{array}$

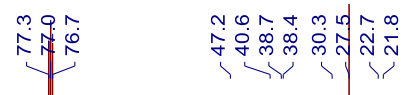

Figure S111. ${ }^{13} \mathbf{C}$ NMR $\left(101 \mathrm{MHz}, \mathrm{CDCl}_{3}\right)$ of $\mathbf{5 f}$ 
<smiles>[Y5]n1c([C@](C)(CBr)CNC(=O)C(C)C)cc2ccccc21</smiles>

$5 g$

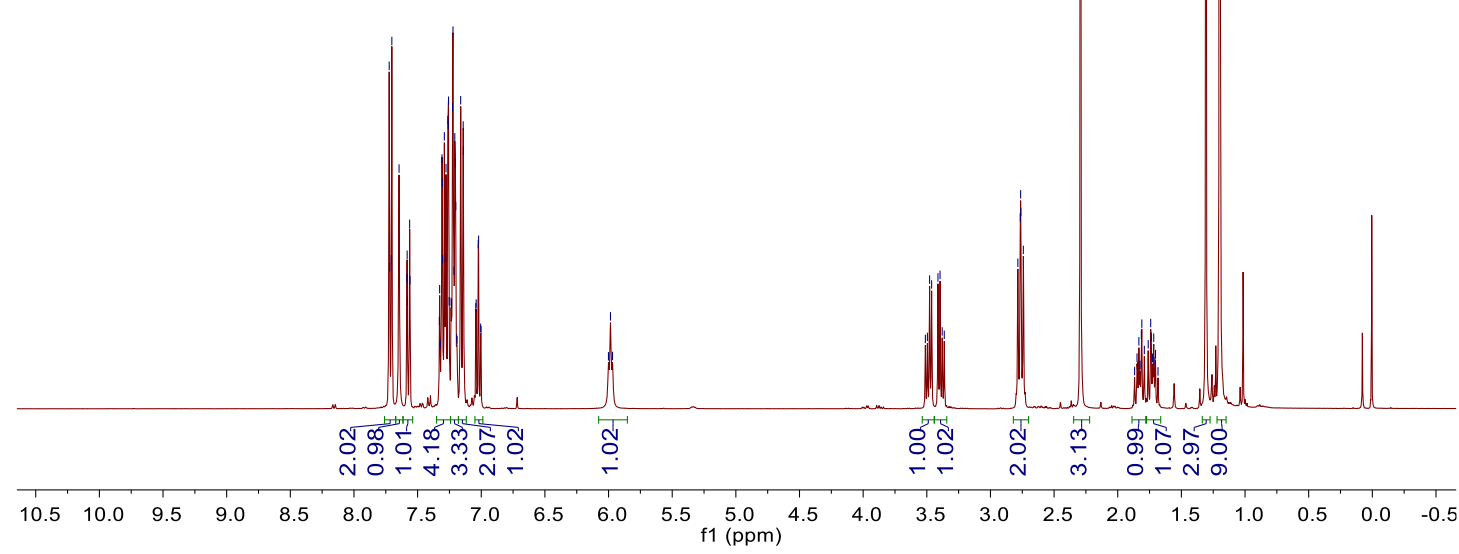

Figure S112. ${ }^{1} \mathbf{H}$ NMR $\left(400 \mathrm{MHz}, \mathrm{CDCl}_{3}\right)$ of $\mathbf{5 g}$

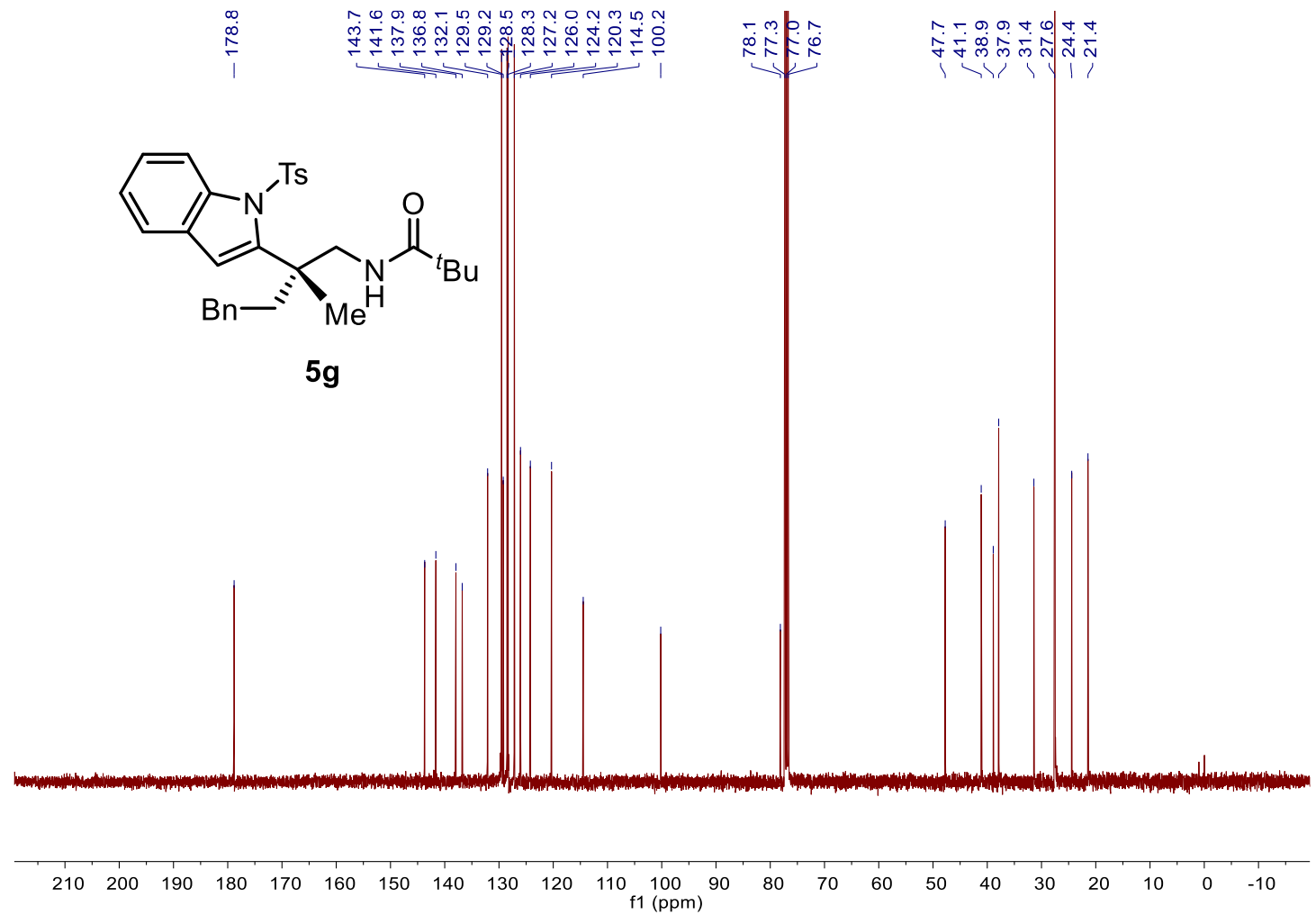

Figure S113. ${ }^{13} \mathbf{C}$ NMR $\left(101 \mathrm{MHz}, \mathrm{CDCl}_{3}\right)$ of $\mathbf{5 g}$ 


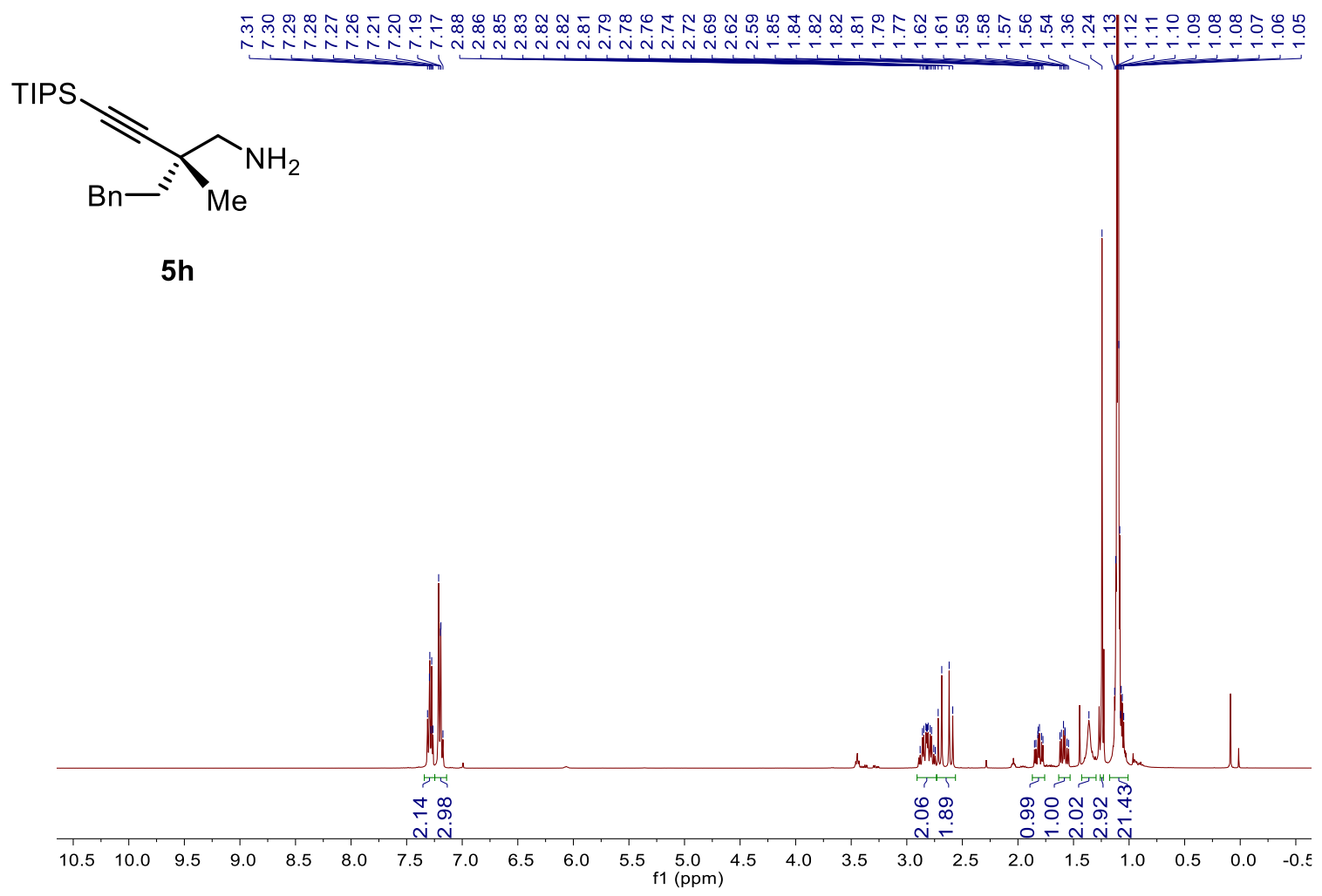

Figure S114. ${ }^{\mathbf{1}} \mathbf{H}$ NMR $\left(400 \mathrm{MHz}, \mathrm{CDCl}_{3}\right)$ of $\mathbf{5 h}$

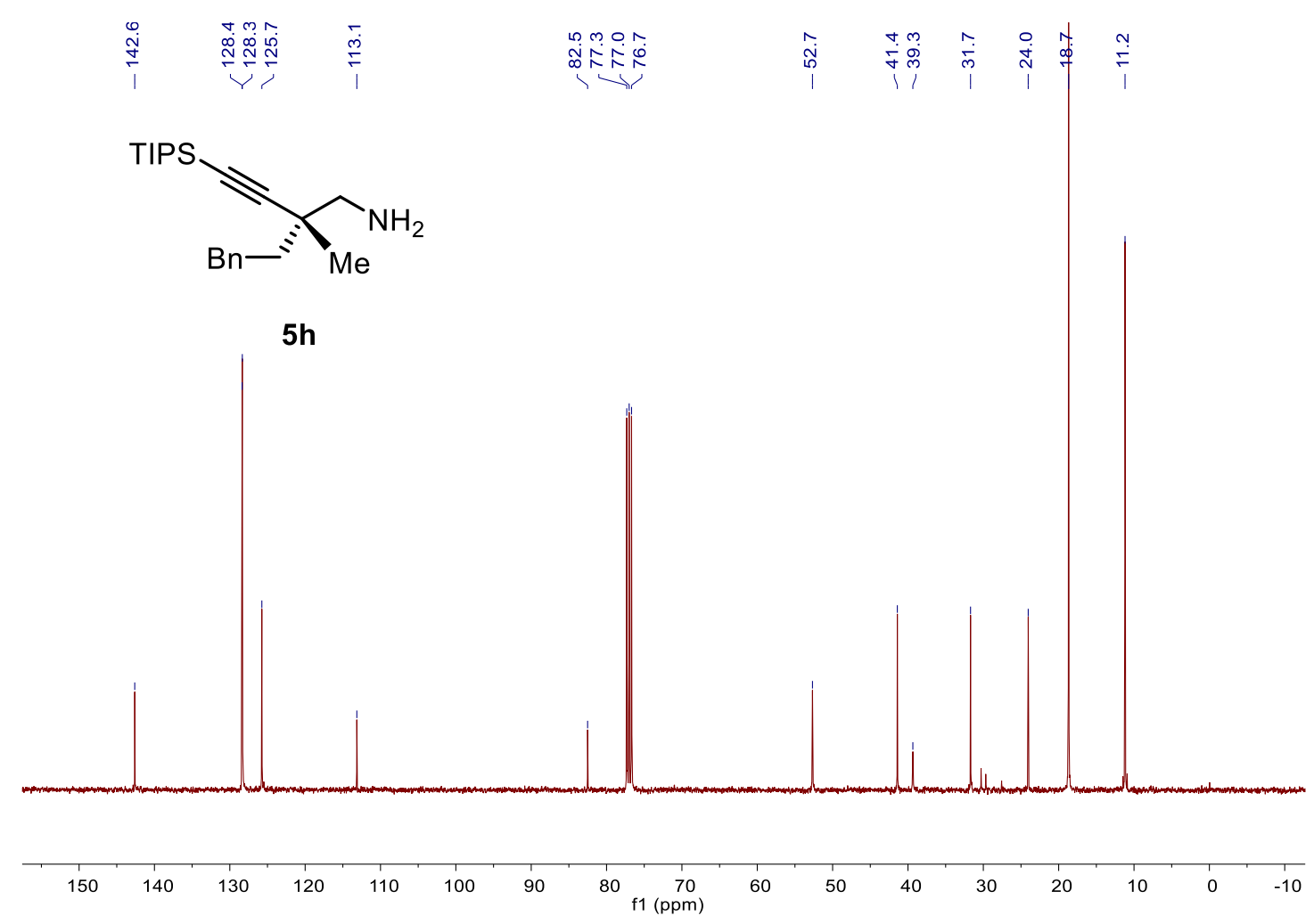

Figure S115. ${ }^{13} \mathbf{C}$ NMR $\left(101 \mathrm{MHz}, \mathrm{CDCl}_{3}\right)$ of $\mathbf{5 h}$ 
<smiles>CC(C)(C)C(=O)N1CCC[C@@](C)(C#C[In]S)C1</smiles>

$5 \mathbf{i}$

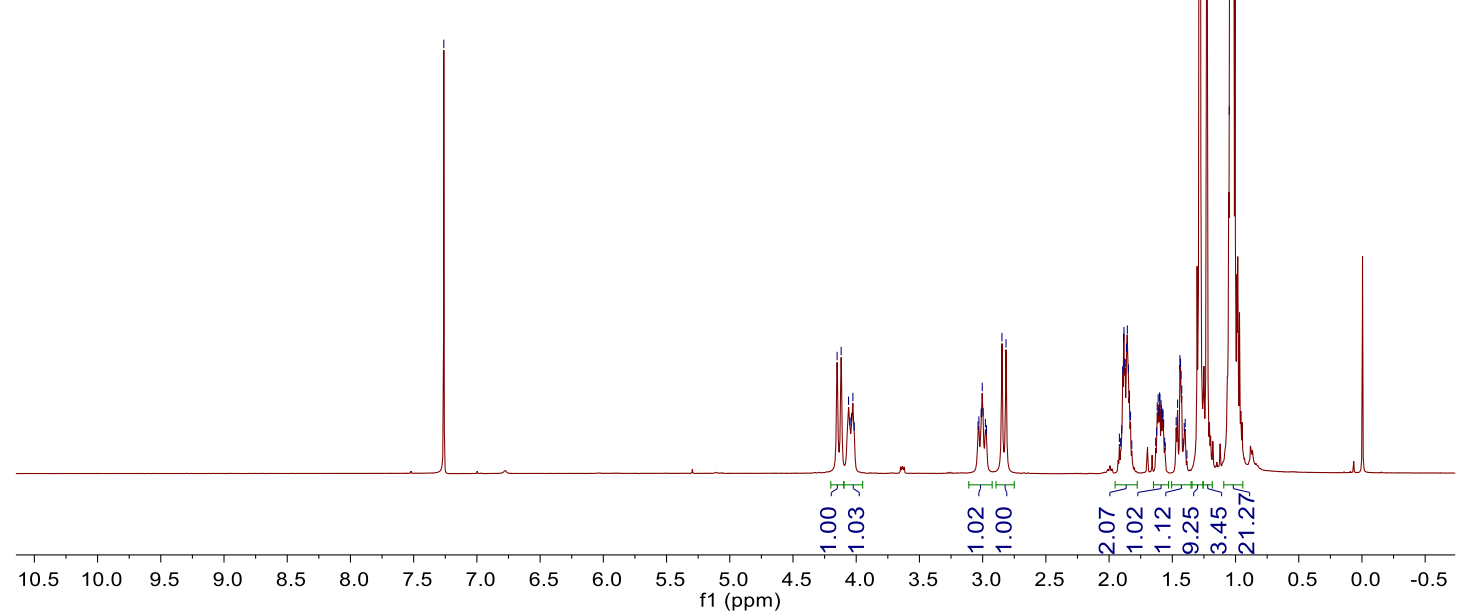

Figure S116. ${ }^{1} \mathbf{H}$ NMR $\left(400 \mathrm{MHz}, \mathrm{CDCl}_{3}\right)$ of $\mathbf{5 i}$<smiles>CC(C)(C)C(=O)N1CCC[C@](C)(C#C[In]C(F)(F)F)C1</smiles>

$5 \mathbf{i}$

$\begin{array}{llllllllllll}210 & 200 & 190 & 180 & 170 & 160 & 150 & 140 & 130 & 120 & 110 \begin{array}{c}100 \\ \mathrm{f} 1(\mathrm{ppm})\end{array}\end{array}$

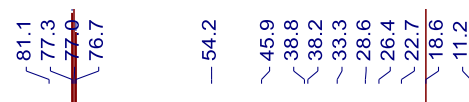

Figure S117. ${ }^{13} \mathbf{C}$ NMR (101 $\left.\mathbf{M H z}, \mathrm{CDCl}_{3}\right)$ of $\mathbf{5 i}$ 
$\operatorname{Ir}(\mathrm{L} 9)(\operatorname{cod}) \mathrm{OTf}$

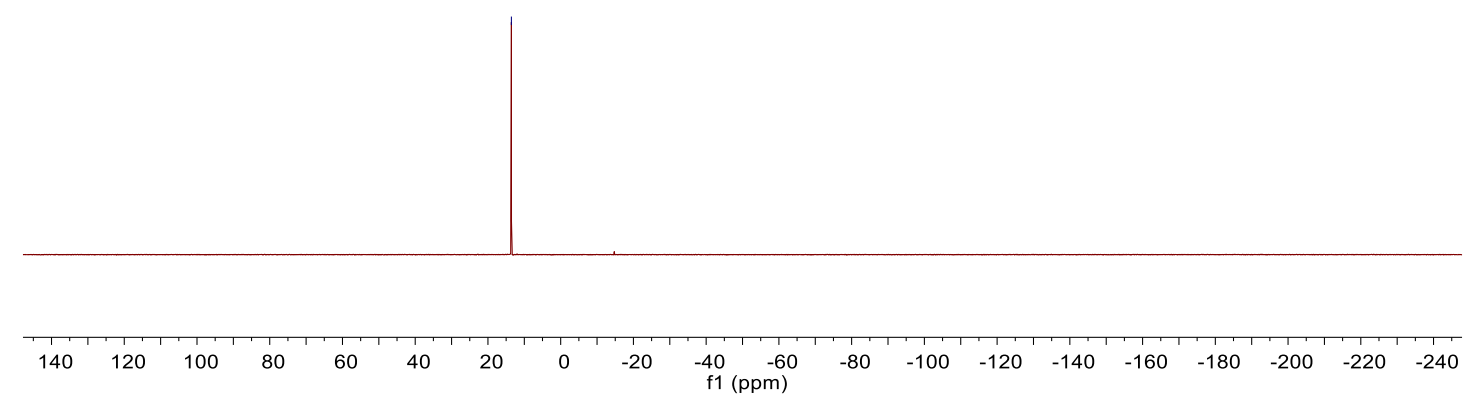

Figure S118. ${ }^{31} \mathbf{P}$ NMR $\left(162 \mathrm{MHz}, \mathrm{CDCl}_{3}\right)$ of complex $\operatorname{Ir}(\mathbf{L 9})(\mathrm{cod}) \mathrm{OTf}$

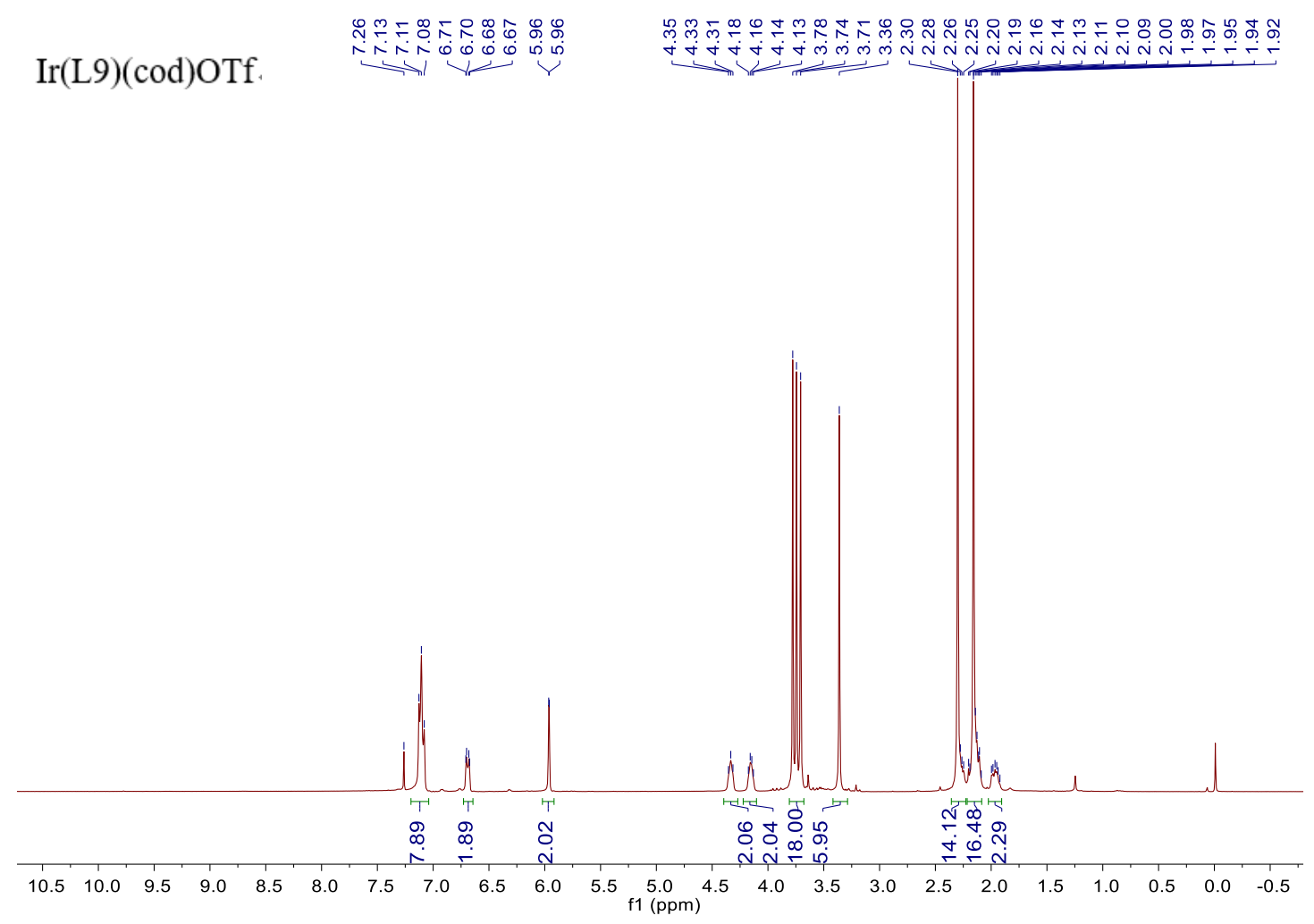

Figure S119. ${ }^{1} \mathbf{H}$ NMR $\left(400 \mathrm{MHz}, \mathrm{CDCl}_{3}\right)$ of complex $\operatorname{Ir}(\mathbf{L 9})(\operatorname{cod}) \mathrm{OTf}$ 


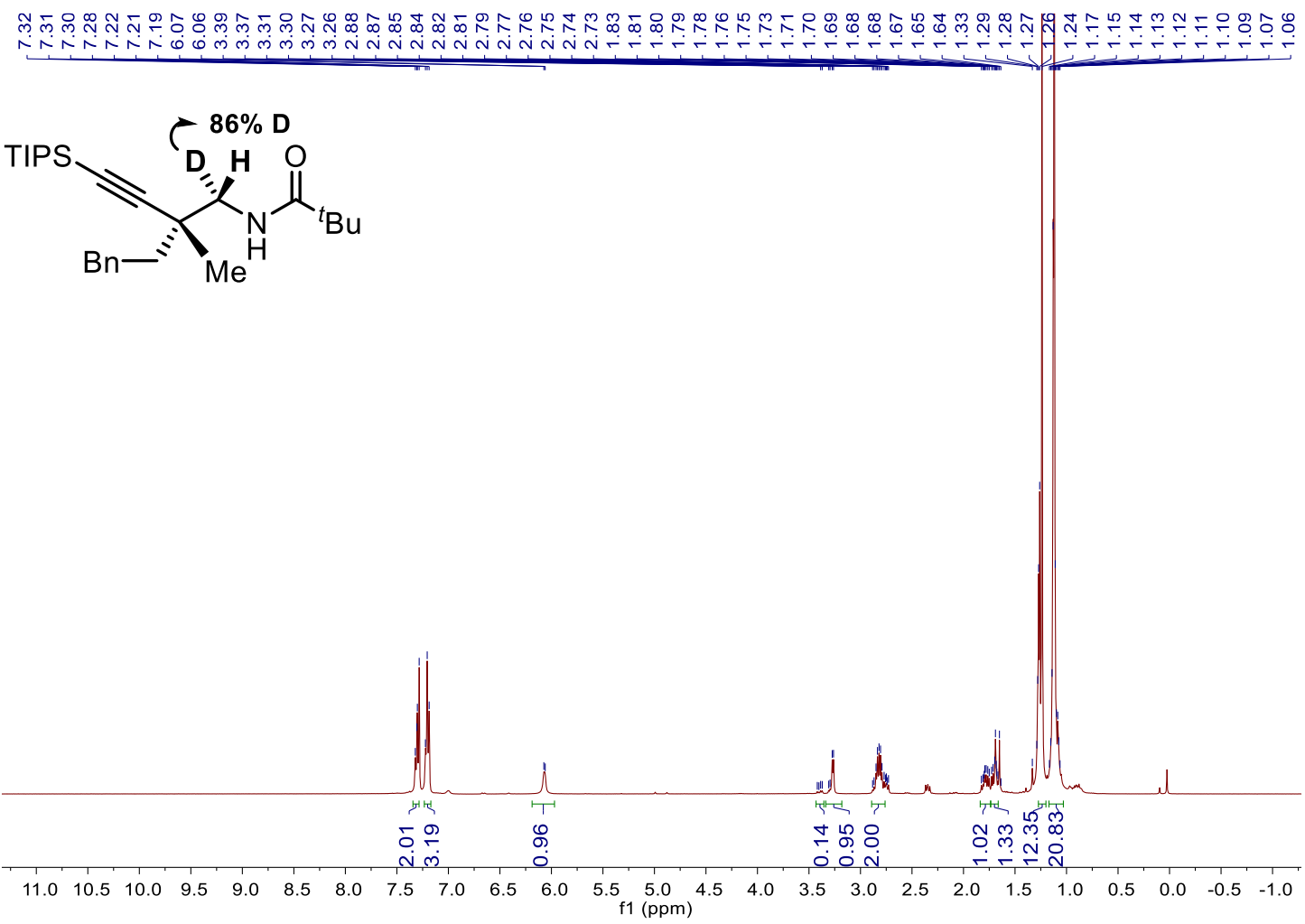

Figure S120. ${ }^{1} \mathbf{H}$ NMR $\left(400 \mathrm{MHz}, \mathrm{CDCl}_{3}\right)$ of $\mathbf{4 a - d}$ starting from D-1a

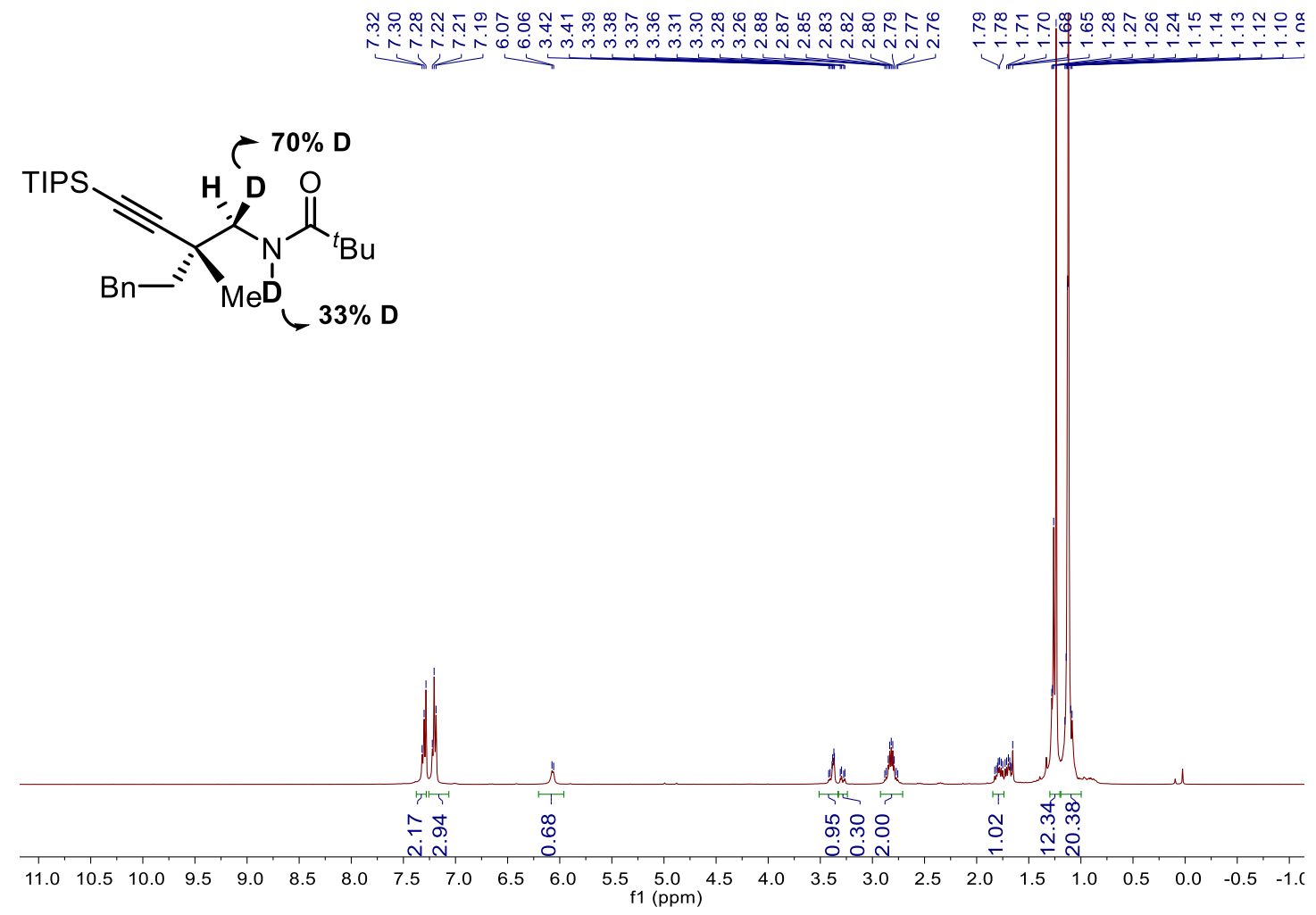

Figure S121. ${ }^{1} \mathbf{H}$ NMR (400 MHz, $\left.\mathrm{CDCl}_{3}\right)$ of $\mathbf{4 a}$-d starting from D-2 


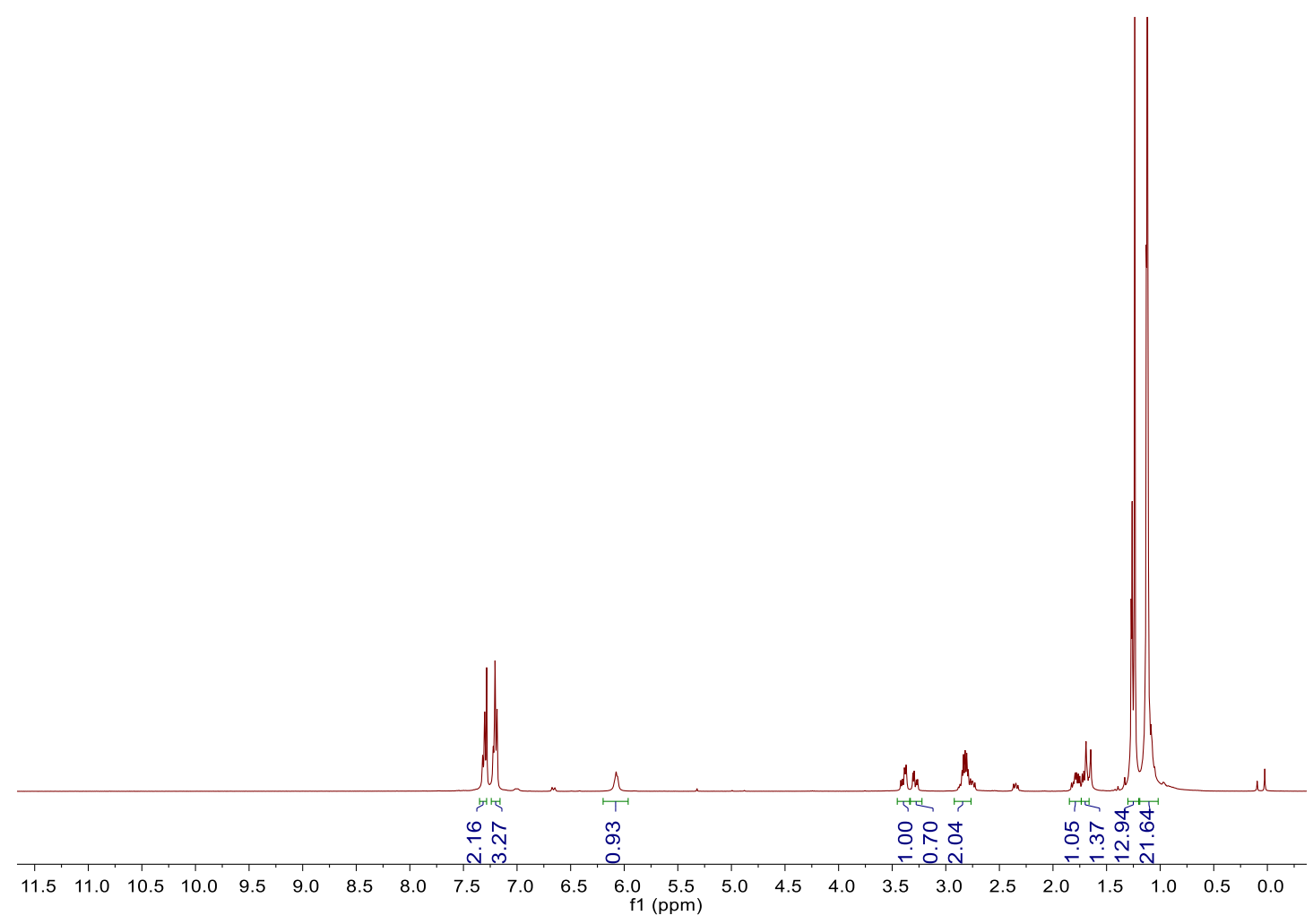

Figure S122. ${ }^{1} \mathbf{H}$ NMR $\left(400 \mathrm{MHz}, \mathrm{CDCl}_{3}\right)$ of $\mathbf{4 a}$-d starting from D-2 and 2 (1:1) 


\section{Copies of HPLC spectra}

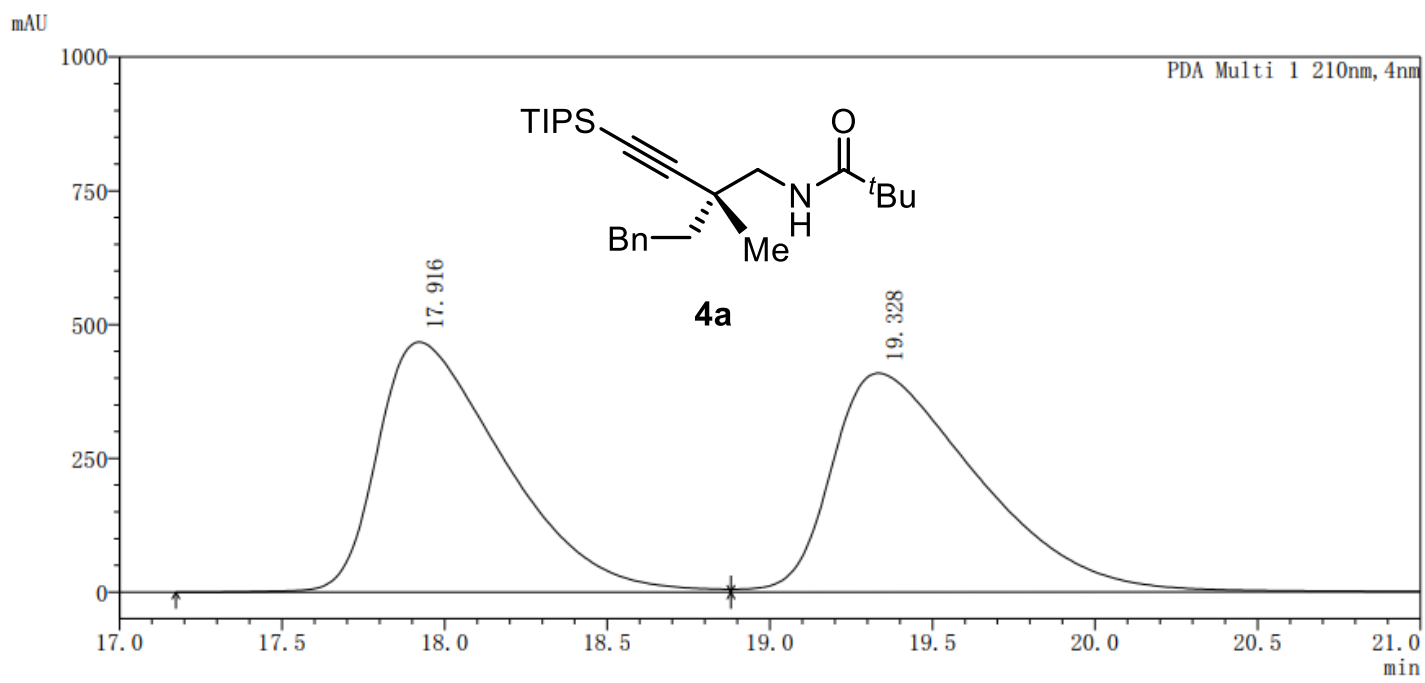

PDA Ch1 210nm

\begin{tabular}{|r|r|r|r|r|}
\hline Peak & Ret. Time & Height & \multicolumn{1}{c|}{ Area } & \multicolumn{1}{c|}{ Area\% } \\
\hline 1 & 17.916 & 467258 & 12652548 & 49.660 \\
\hline 2 & 19.328 & 408816 & 12825744 & 50.340 \\
\hline
\end{tabular}

Figure S123. HPLC record for racemic 4a

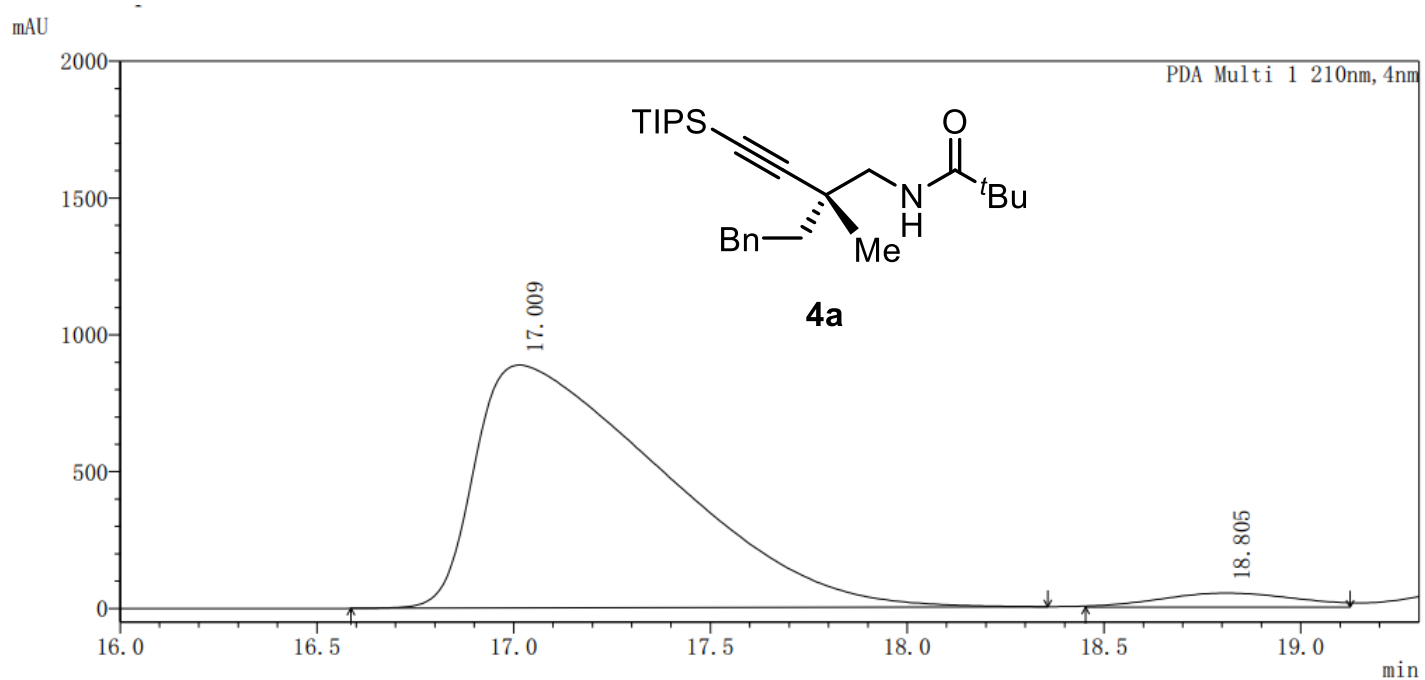

PDA Ch1 210nm

\begin{tabular}{|r|r|r|r|r|}
\hline Peak & Ret. Time & Height & \multicolumn{1}{c|}{ Area } & \multicolumn{1}{c|}{ Area\% } \\
\hline 1 & 17.009 & 888076 & 29702731 & 95.993 \\
\hline 2 & 18.805 & 51068 & 1239868 & 4.007 \\
\hline
\end{tabular}

Figure S124. HPLC record for chiral 4a 


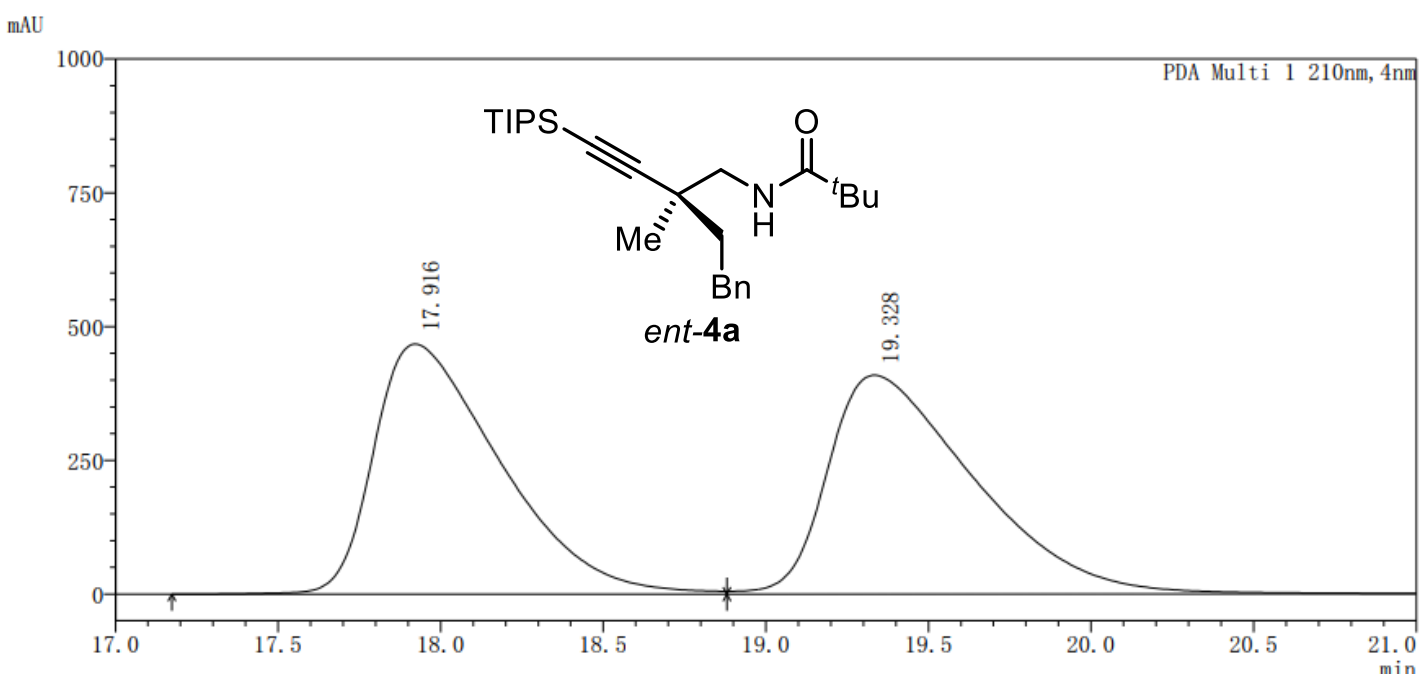

PDA Ch1 210nm

\begin{tabular}{|r|r|r|c|r|}
\hline Peak & Ret. Time & Height & Area & \multicolumn{1}{c|}{ Area\% } \\
\hline 1 & 17.916 & 467258 & 12652548 & 49.660 \\
\hline 2 & 19.328 & 408816 & 12825744 & 50.340 \\
\hline
\end{tabular}

Figure S125. HPLC record for racemic ent-4a

$\mathrm{mAU}$

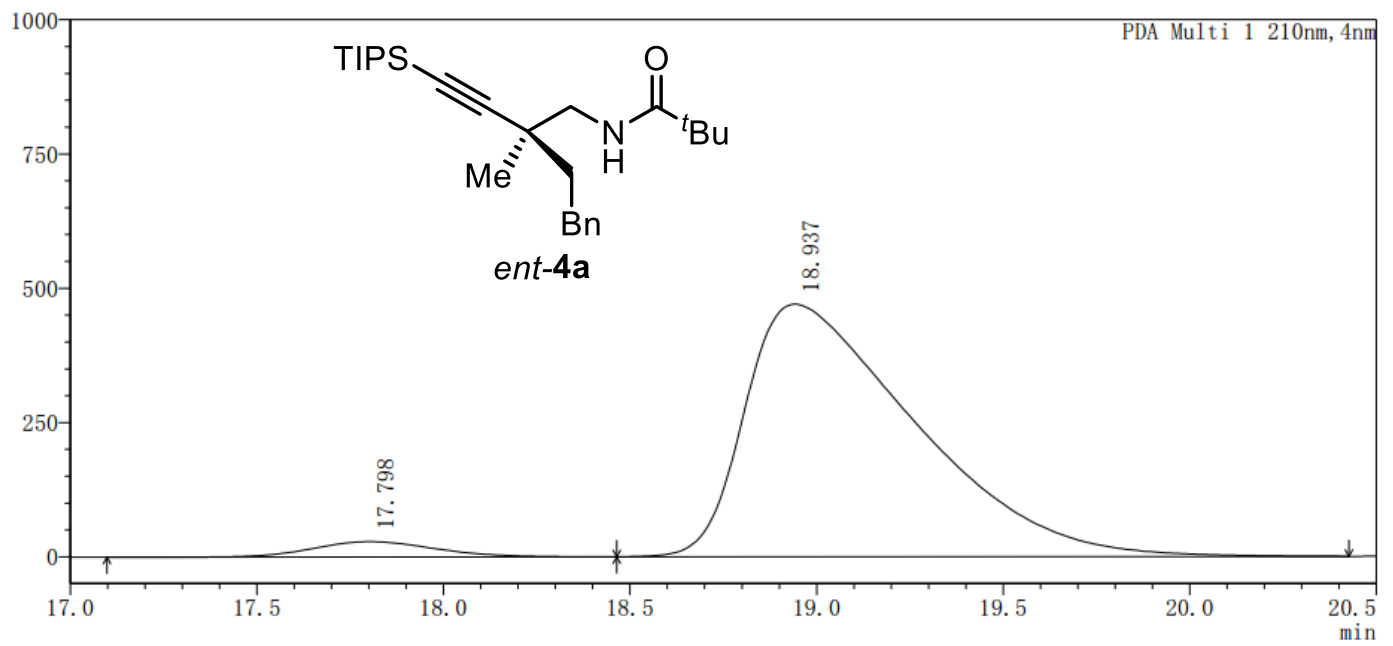

PDA Ch1 210nm

\begin{tabular}{|r|r|r|r|r|}
\hline Peak & Ret. Time & \multicolumn{1}{c|}{ Height } & \multicolumn{1}{c|}{ Area } & \multicolumn{1}{c|}{ Area\% } \\
\hline 1 & 17.798 & 28597 & 650607 & 4.169 \\
\hline 2 & 18.937 & 470183 & 14956955 & 95.831 \\
\hline
\end{tabular}

Figure S126. HPLC record for racemic ent-4a 


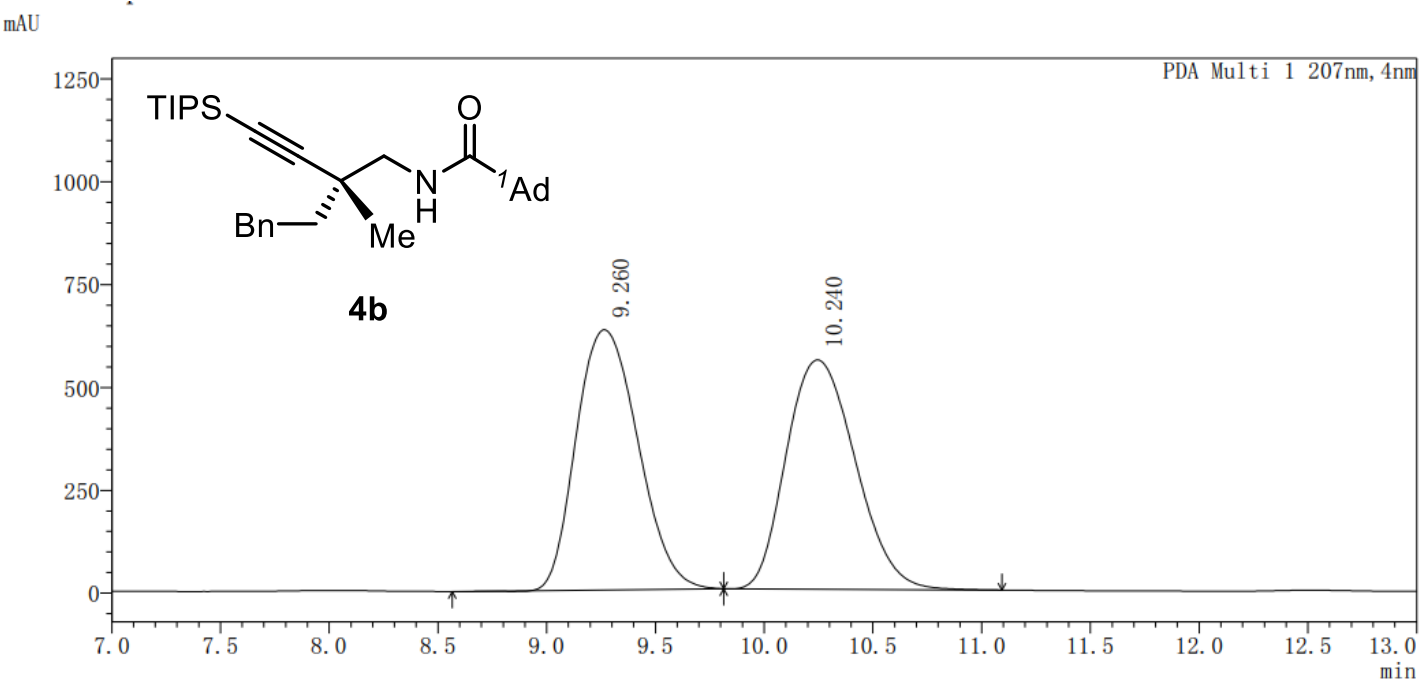

PDA Ch1 $207 \mathrm{~nm}$
\begin{tabular}{|r|r|r|c|c|}
\hline Peak & Ret. Time & Height & Area & Area $\%$ \\
\hline 1 & 9.260 & 632702 & 12515447 & 50.343 \\
\hline 2 & 10.240 & 557185 & 12345063 & 49.657 \\
\hline
\end{tabular}

Figure S127. HPLC record for racemic $4 b$

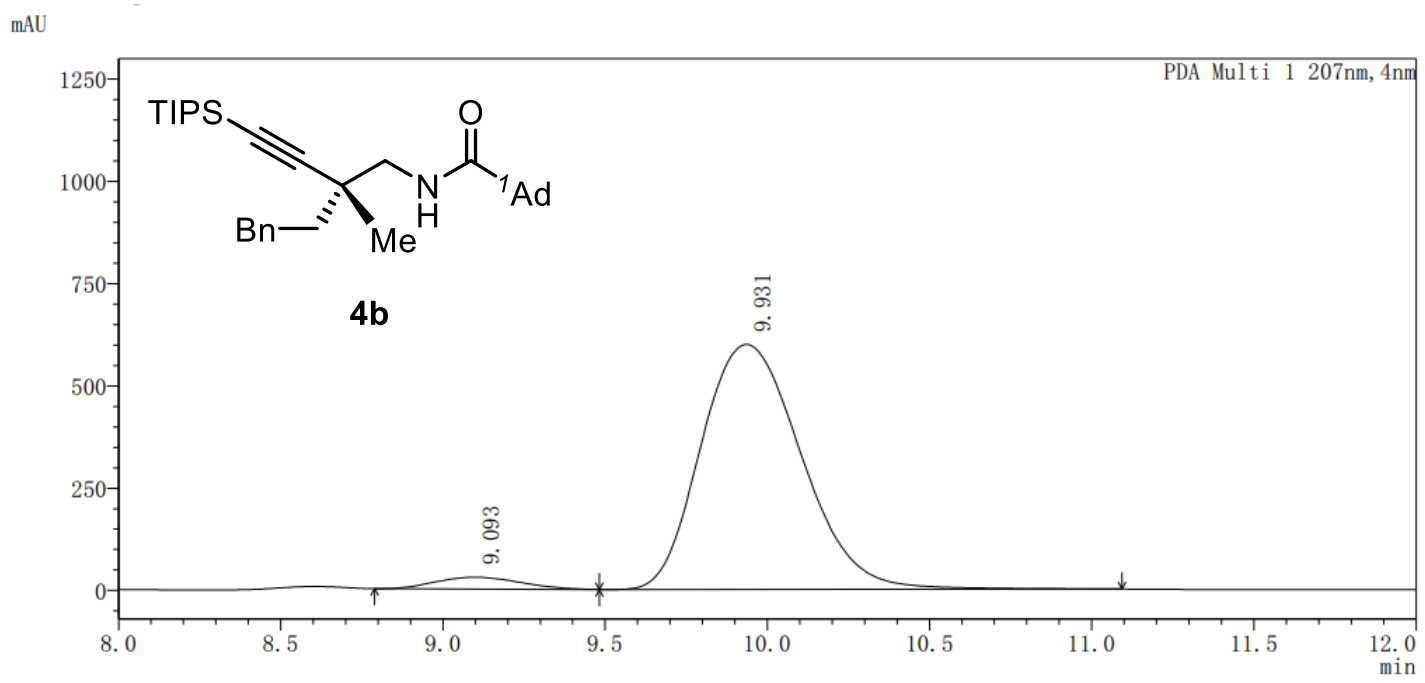

PDA Ch1 207nm

\begin{tabular}{|r|r|r|r|r|}
\hline Peak & Ret. Time & Height & \multicolumn{1}{c|}{ Area } & \multicolumn{1}{c|}{ Area\% } \\
\hline 1 & 9.093 & 28932 & 526949 & 3.922 \\
\hline 2 & 9.931 & 598842 & 12908201 & 96.078 \\
\hline
\end{tabular}

Figure S128. HPLC record for chiral 4b 


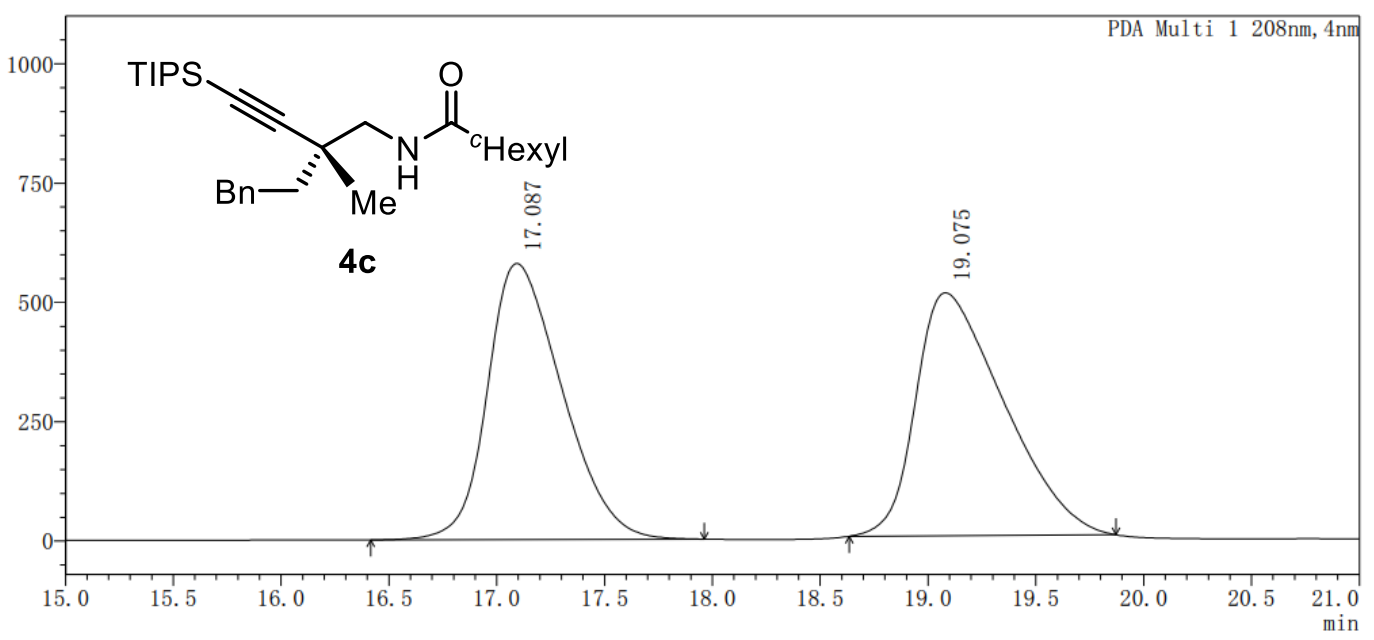

PDA Ch1 208nm

\begin{tabular}{|r|r|r|r|r|}
\hline Peak & Ret. Time & Height & \multicolumn{1}{c|}{ Area } & \multicolumn{1}{c|}{ Area\% } \\
\hline 1 & 17.087 & 578605 & 13961416 & 49.076 \\
\hline 2 & 19.075 & 508960 & 14487176 & 50.924 \\
\hline
\end{tabular}

Figure S129. HPLC record for racemic 4c

mAU

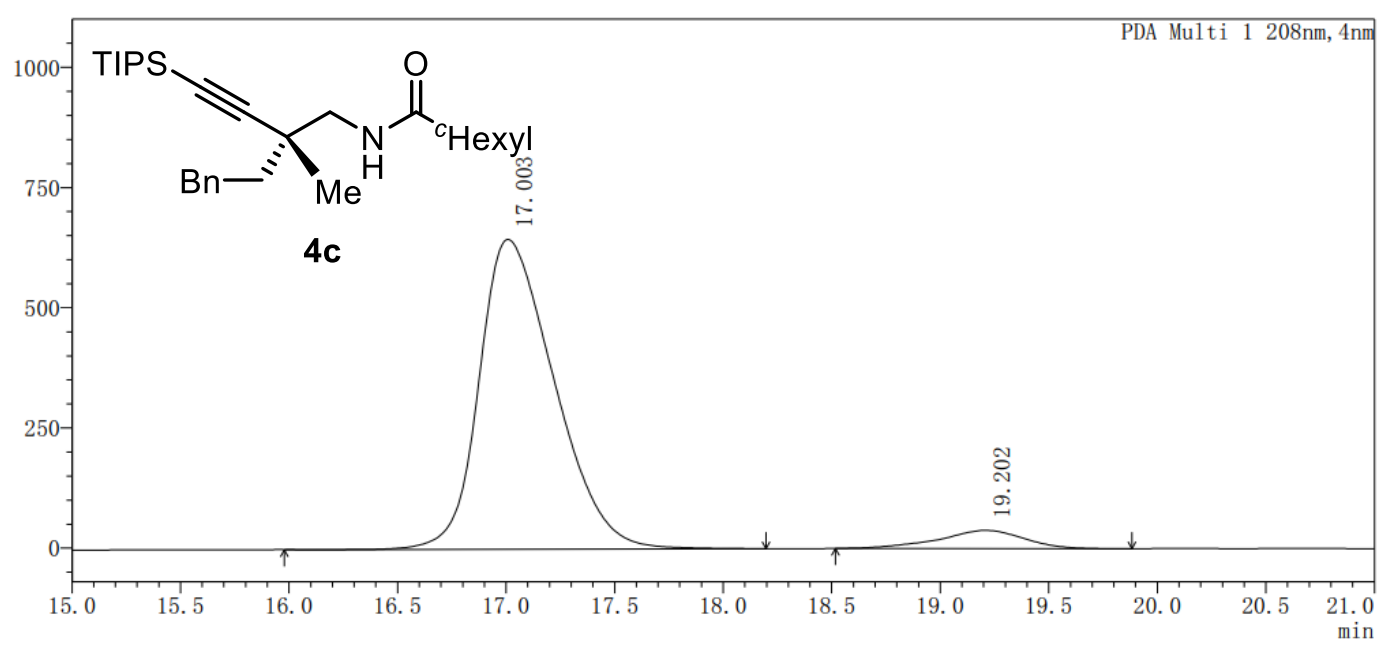

PDA Ch1 208nm

\begin{tabular}{|r|r|r|r|r|}
\hline Peak & Ret. Time & \multicolumn{1}{c|}{ Height } & \multicolumn{1}{c|}{ Area } & \multicolumn{1}{c|}{ Area\% } \\
\hline 1 & 17.003 & 644276 & 15547561 & 93.785 \\
\hline 2 & 19.202 & 37856 & 1030363 & 6.215 \\
\hline
\end{tabular}

Figure S130. HPLC record for chiral 4c 


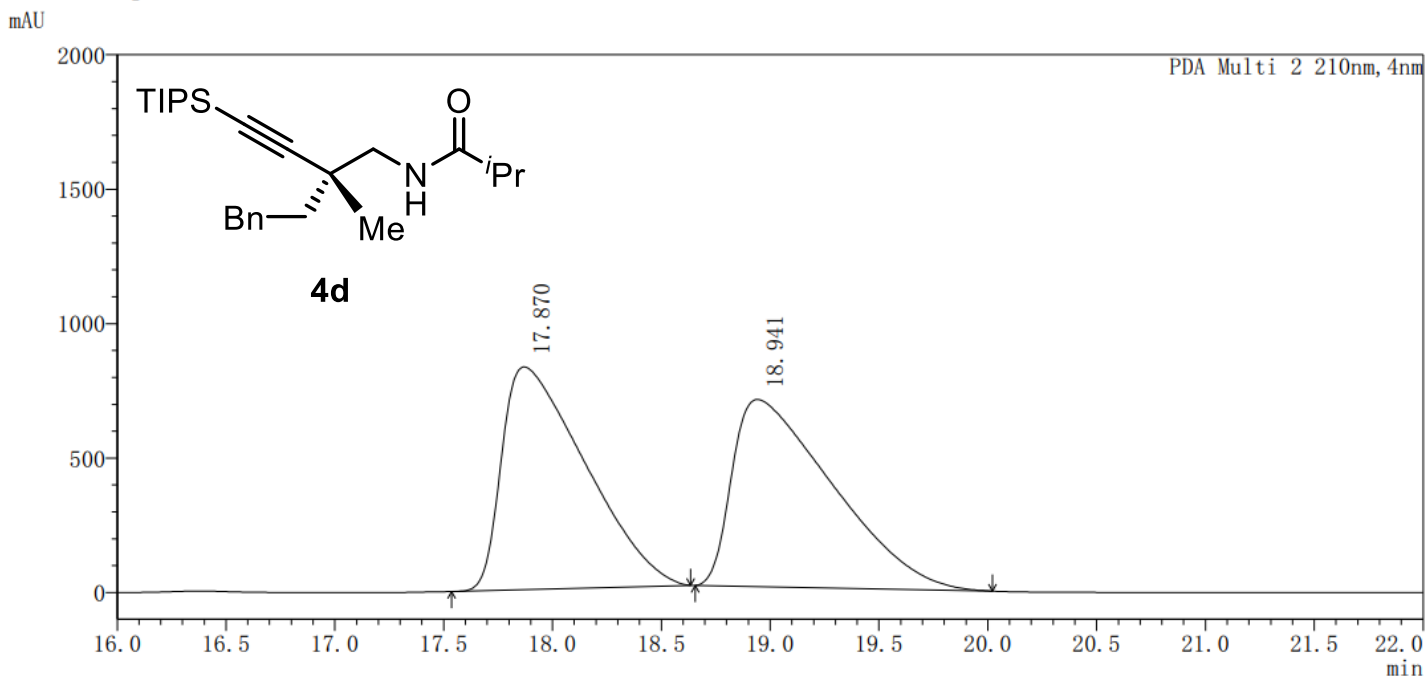

PDA Ch2 210nm

\begin{tabular}{|r|r|r|r|r|}
\hline Peak & Ret. Time & Height & Area & \multicolumn{1}{c|}{ Area\% } \\
\hline 1 & 17.870 & 828772 & 22119255 & 49.750 \\
\hline 2 & 18.941 & 696094 & 22341115 & 50.250 \\
\hline
\end{tabular}

Figure S131. HPLC record for racemic 4d

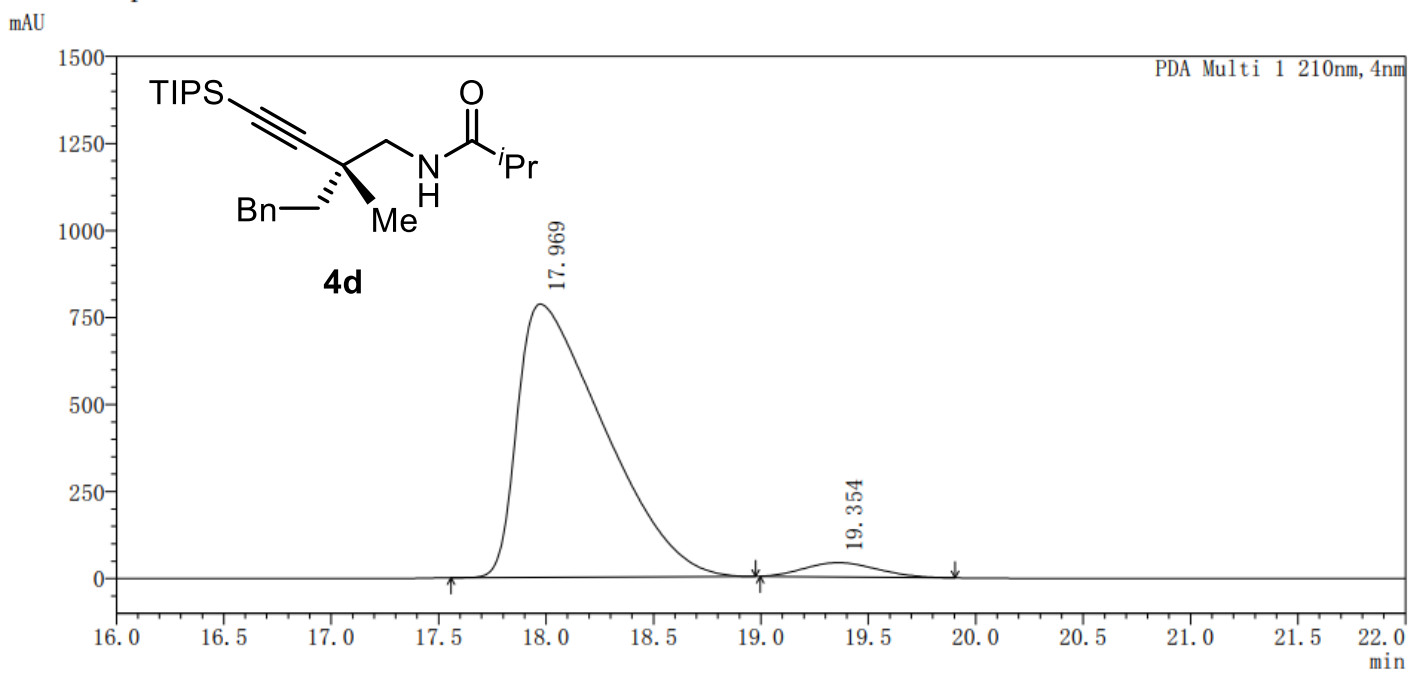

PDA Ch1 210nm
\begin{tabular}{|r|r|r|r|r|}
\hline Peak & Ret. Time & Height & \multicolumn{1}{c|}{ Area } & \multicolumn{1}{c|}{ Area\% } \\
\hline 1 & 17.969 & 785285 & 22028884 & 95.802 \\
\hline 2 & 19.354 & 40730 & 965282 & 4.198 \\
\hline
\end{tabular}

Figure S132. HPLC record for chiral 4d 
$\mathrm{mAU}$

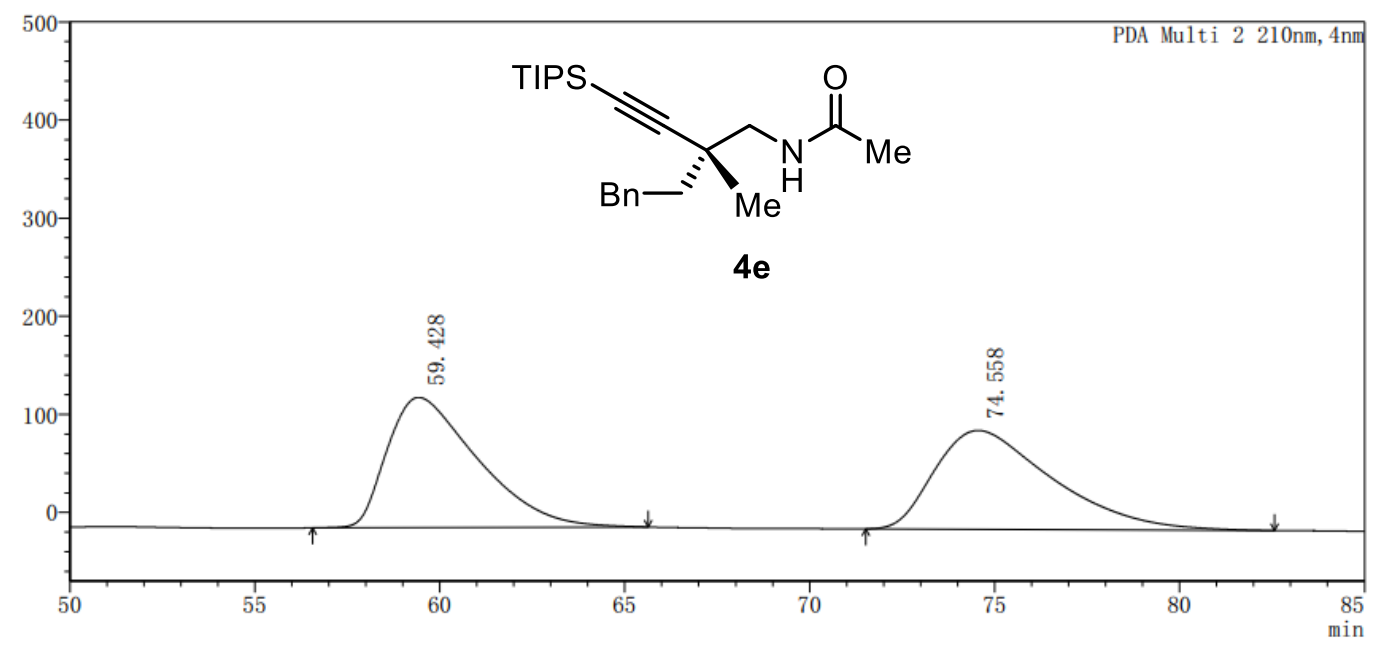

PDA Ch2 210nm
\begin{tabular}{|r|r|r|c|c|}
\hline Peak & Ret. Time & Height & Area & Area $\%$ \\
\hline 1 & 59.428 & 132736 & 22581773 & 50.296 \\
\hline 2 & 74.558 & 100815 & 22316191 & 49.704 \\
\hline
\end{tabular}

Figure S133. HPLC record for racemic 4e

$\mathrm{mAU}$

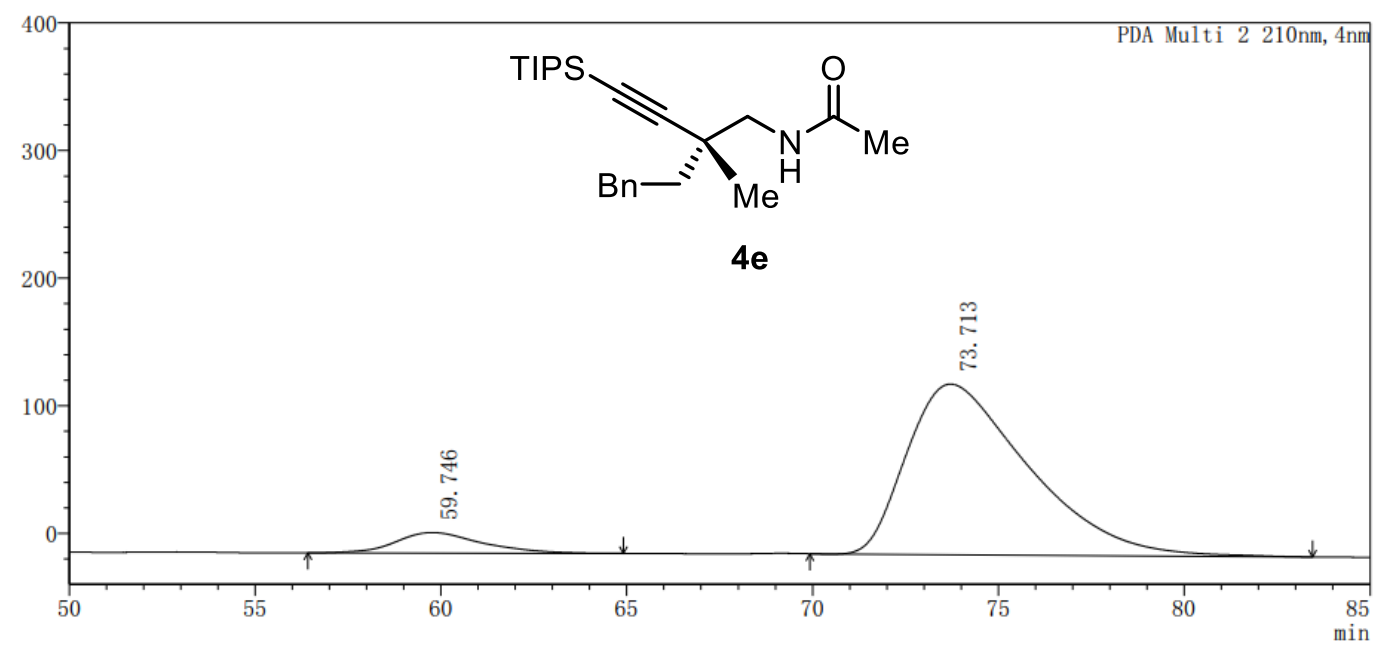

PDA Ch2 210nm

\begin{tabular}{|r|r|r|r|r|}
\hline Peak & Ret. Time & \multicolumn{1}{c|}{ Height } & \multicolumn{1}{|c|}{ Area } & \multicolumn{1}{c|}{ Area\% } \\
\hline 1 & 59.746 & 16104 & 2600227 & 7.864 \\
\hline 2 & 73.713 & 133720 & 30466239 & 92.136 \\
\hline
\end{tabular}

Figure S134. HPLC record for chiral 4e 
mAU

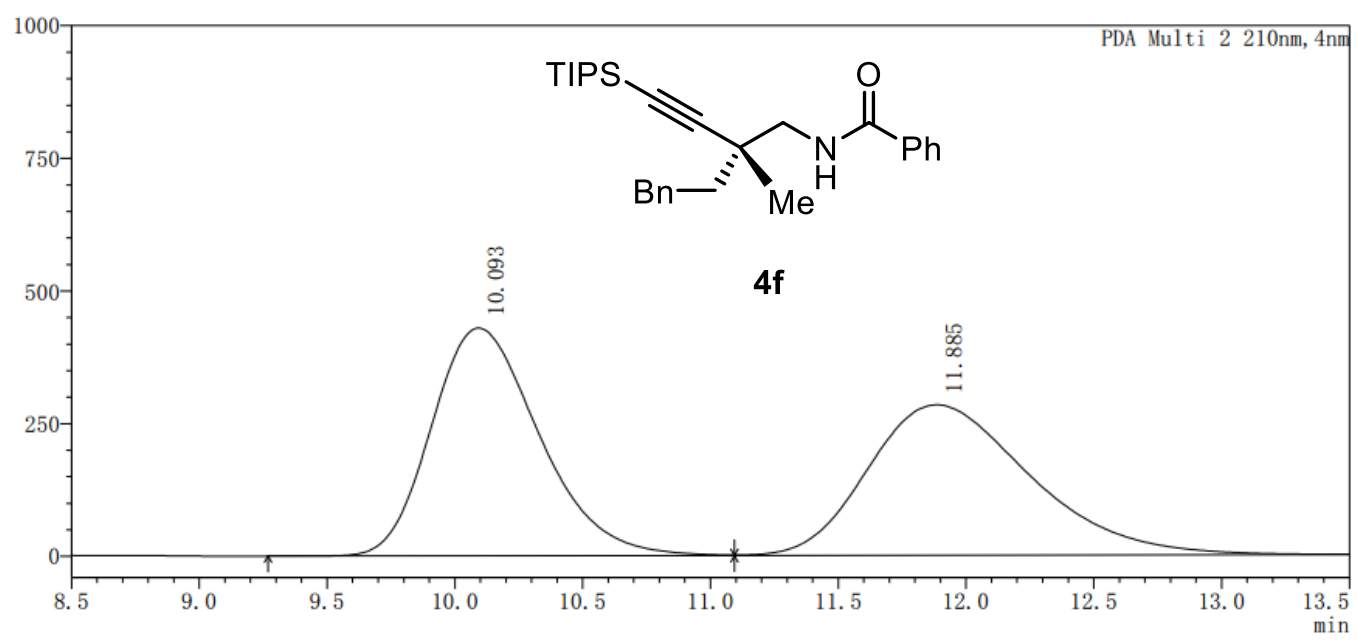

PDA Ch2 210nm

\begin{tabular}{|r|r|r|c|c|}
\hline Peak & Ret. Time & \multicolumn{1}{c|}{ Height } & Area & \multicolumn{1}{c|}{ Area\% } \\
\hline 1 & 10.093 & 429234 & 12606716 & 50.079 \\
\hline 2 & 11.885 & 283449 & 12567083 & 49.921 \\
\hline
\end{tabular}

Figure S135. HPLC record for racemic $4 f$

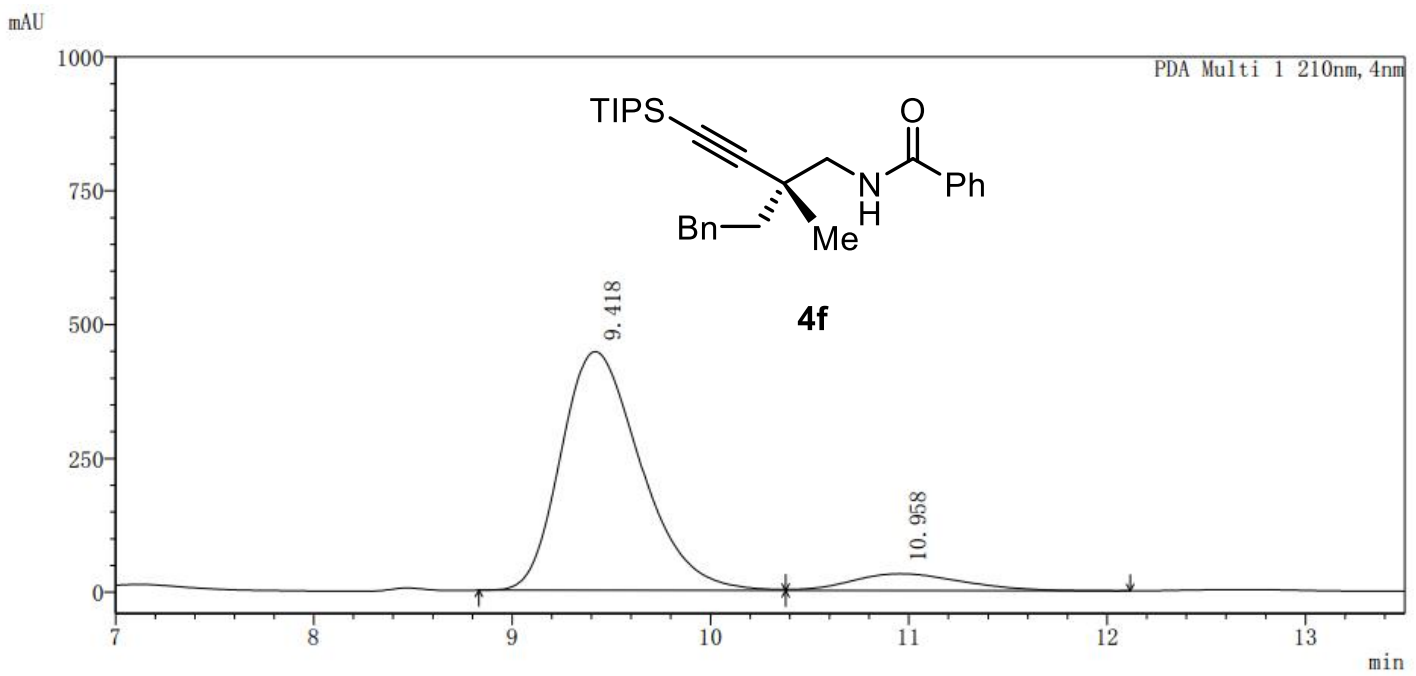

PDA Ch1 210nm

\begin{tabular}{|r|r|r|r|r|}
\hline Peak & Ret. Time & \multicolumn{1}{c|}{ Height } & \multicolumn{1}{c|}{ Area } & \multicolumn{1}{c|}{ Area\% } \\
\hline 1 & 9.418 & 446105 & 12513334 & 91.032 \\
\hline 2 & 10.958 & 31555 & 1232807 & 8.968 \\
\hline
\end{tabular}

Figure S136. HPLC record for chiral $4 f$ 
$\mathrm{mAU}$

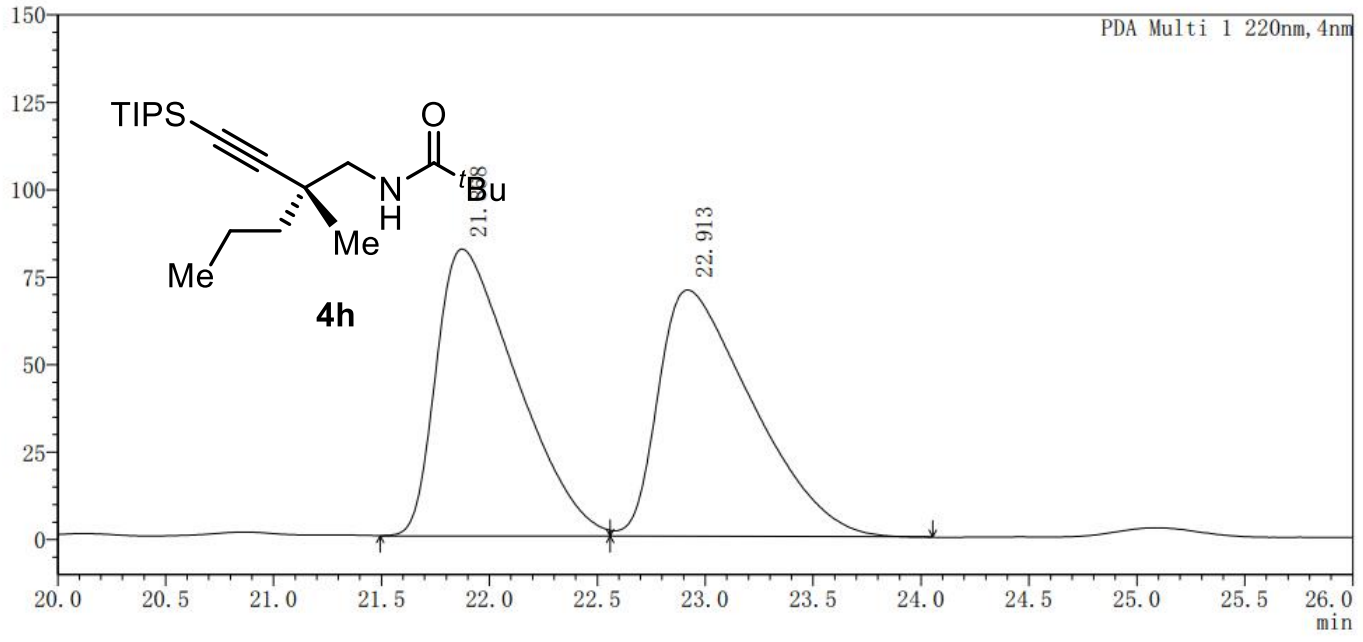

PDA Ch1 220nm

\begin{tabular}{|r|r|r|r|r|}
\hline Peak & Ret. Time & Height & \multicolumn{1}{c|}{ Area } & \multicolumn{1}{c|}{ Area\% } \\
\hline 1 & 21.868 & 82039 & 2132581 & 49.962 \\
\hline 2 & 22.913 & 70443 & 2135823 & 50.038 \\
\hline
\end{tabular}

Figure S137. HPLC record for racemic $4 \mathrm{~h}$

$\mathrm{mAU}$

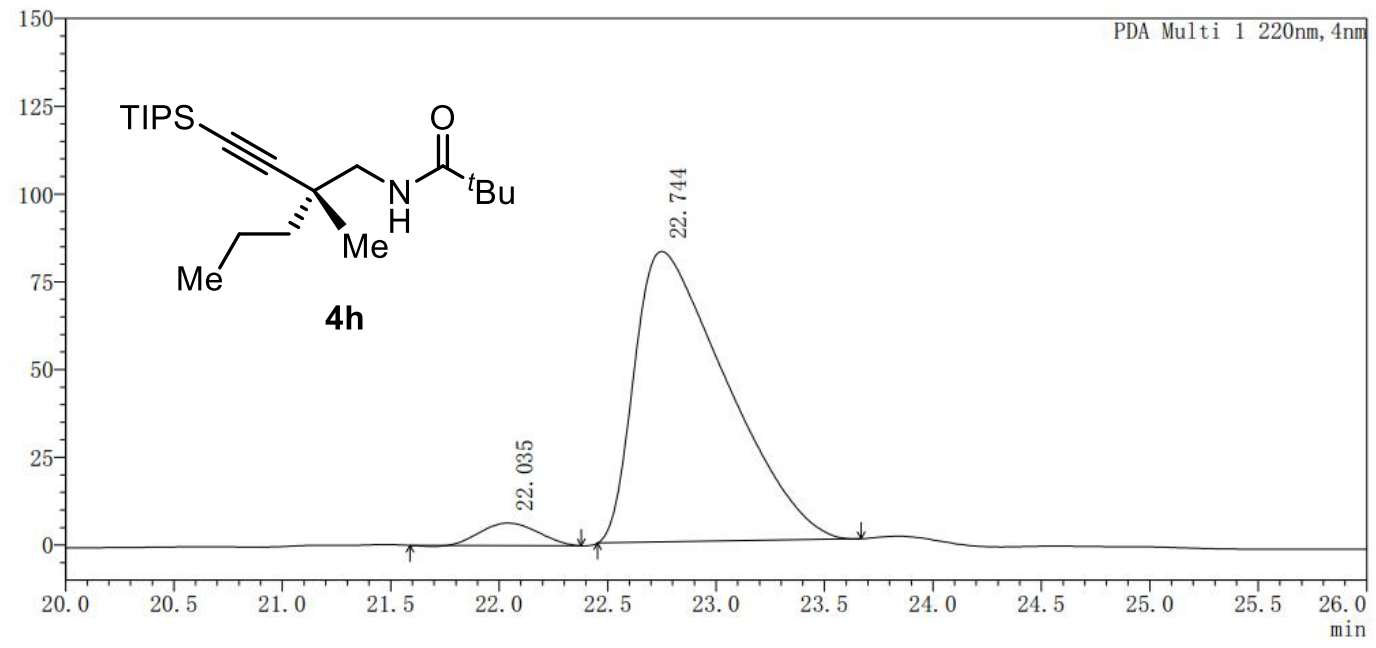

PDA Ch1 220nm

\begin{tabular}{|r|r|r|r|r|}
\hline Peak & Ret. Time & Height & \multicolumn{1}{c|}{ Area } & \multicolumn{1}{c|}{ Area\% } \\
\hline 1 & 22.035 & 6446 & 118209 & 4.607 \\
\hline 2 & 22.744 & 82771 & 2447756 & 95.393 \\
\hline
\end{tabular}

Figure S138. HPLC record for chiral $4 \mathrm{~h}$ 
$\mathrm{mAU}$

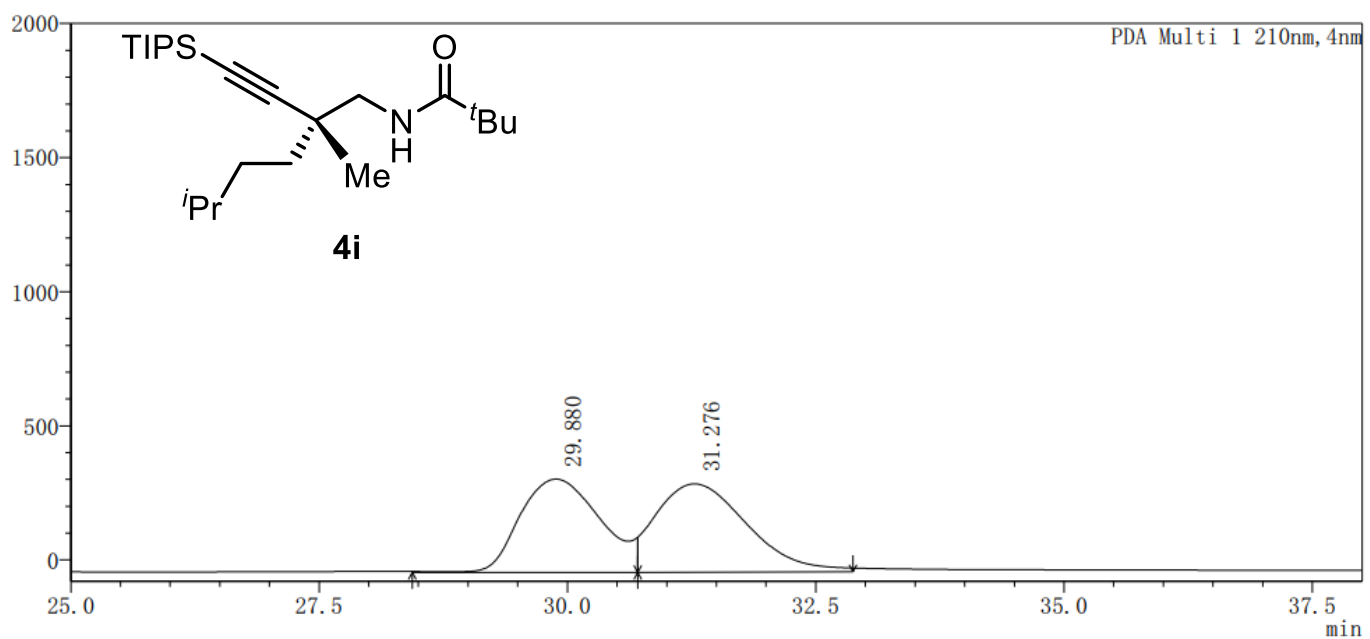

PDA Ch1 210nm

\begin{tabular}{|r|r|r|c|c|}
\hline Peak & Ret. Time & \multicolumn{1}{c|}{ Height } & Area & \multicolumn{1}{c|}{ Area\% } \\
\hline 1 & 29.880 & 347607 & 19658051 & 48.497 \\
\hline 2 & 31.276 & 329306 & 20876204 & 51.503 \\
\hline
\end{tabular}

Figure S139. HPLC record for racemic $4 i$

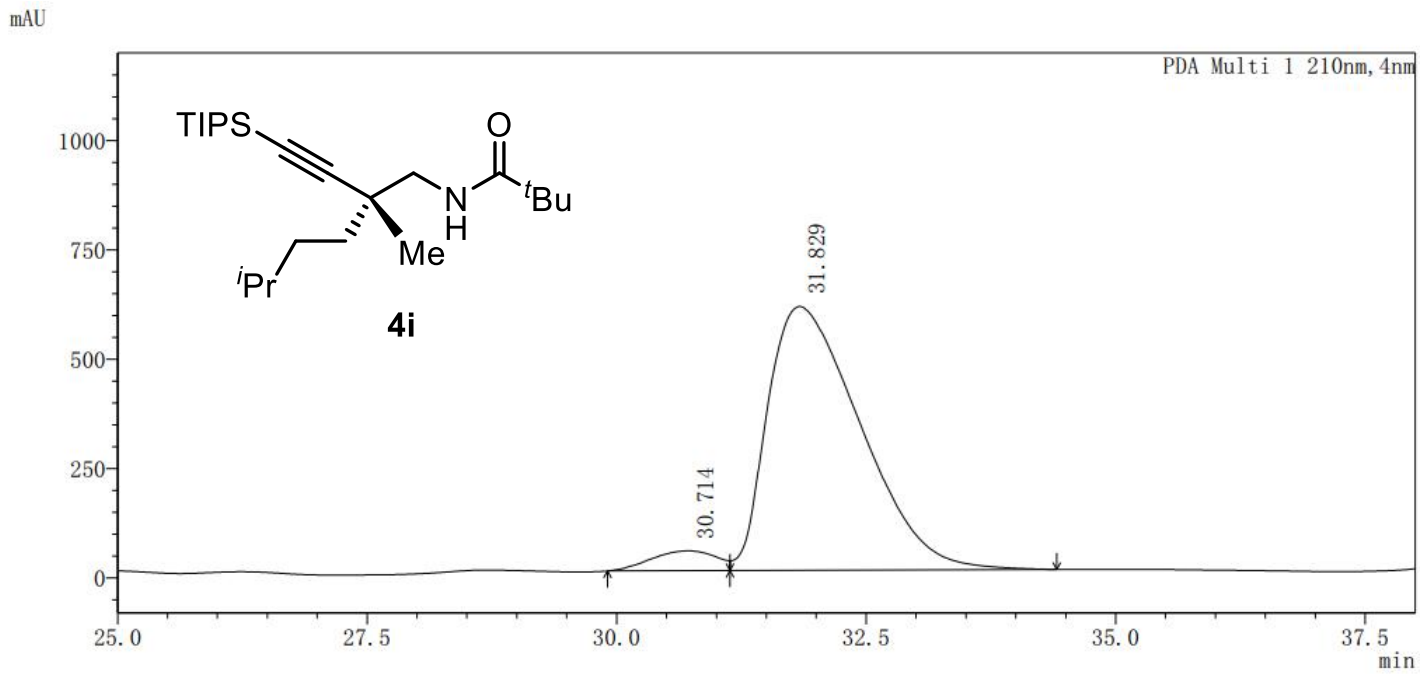

PDA Ch1 210nm

\begin{tabular}{|r|r|r|r|r|}
\hline Peak & Ret. Time & \multicolumn{1}{c|}{ Height } & \multicolumn{1}{|c|}{ Area } & \multicolumn{1}{c|}{ Area\% } \\
\hline 1 & 30.714 & 45394 & 2055337 & 4.996 \\
\hline 2 & 31.829 & 602913 & 39083285 & 95.004 \\
\hline
\end{tabular}

Figure S140. HPLC record for chiral 4i 
$\mathrm{mAU}$

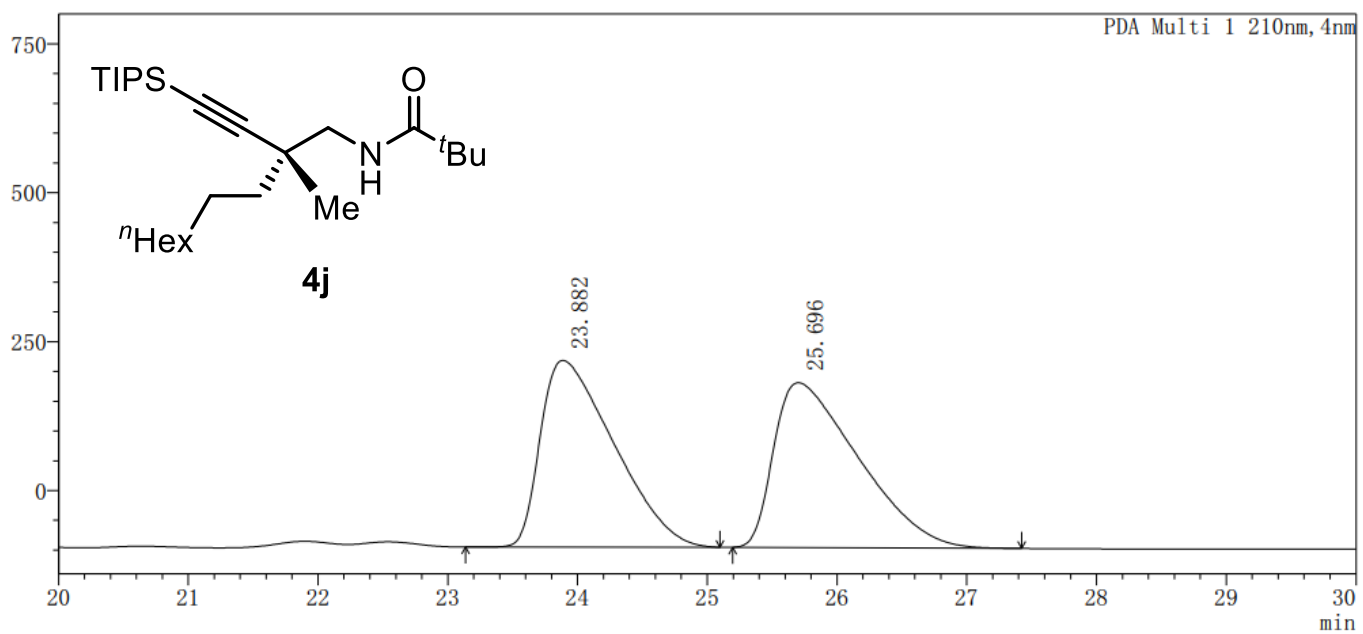

PDA Ch1 210nm

\begin{tabular}{|r|r|r|c|c|}
\hline Peak & Ret. Time & \multicolumn{1}{c|}{ Height } & \multicolumn{1}{c|}{ Area } & \multicolumn{1}{c|}{ Area\% } \\
\hline 1 & 23.882 & 313548 & 12336010 & 49.582 \\
\hline 2 & 25.696 & 276916 & 12544024 & 50.418 \\
\hline
\end{tabular}

Figure S141. HPLC record for racemic $4 \mathbf{j}$

mAU

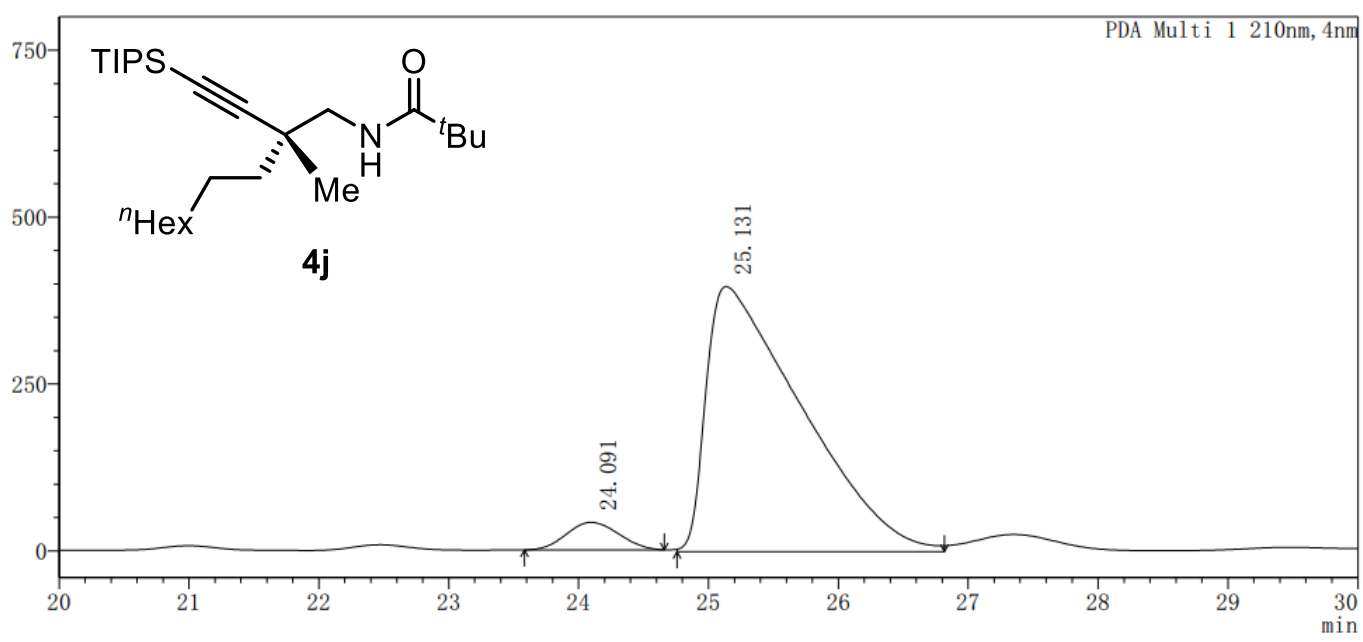

PDA Ch1 210nm

\begin{tabular}{|r|r|r|r|r|}
\hline Peak & Ret. Time & \multicolumn{1}{c|}{ Height } & \multicolumn{1}{c|}{ Area } & \multicolumn{1}{c|}{ Area\% } \\
\hline 1 & 24.091 & 41225 & 1151216 & 5.358 \\
\hline 2 & 25.131 & 396956 & 20336520 & 94.642 \\
\hline
\end{tabular}

Figure S142. HPLC record for chiral $4 \mathbf{j}$ 


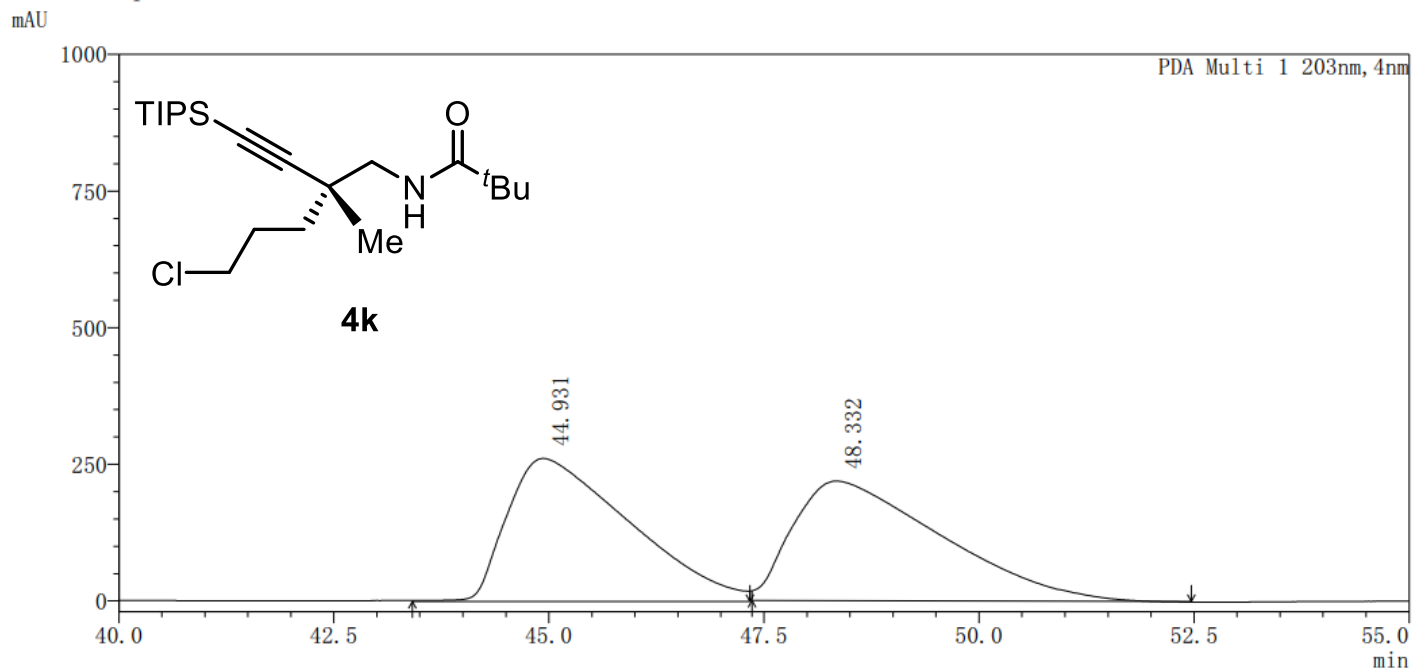

PDA Ch1 203nm
\begin{tabular}{|r|r|r|c|r|}
\hline Peak & Ret. Time & Height & Area & \multicolumn{1}{c|}{ Area $\%$} \\
\hline 1 & 44.931 & 261886 & 25944776 & 49.737 \\
\hline 2 & 48.332 & 218956 & 26219554 & 50.263 \\
\hline
\end{tabular}

Figure S143. HPLC record for racemic $4 k$

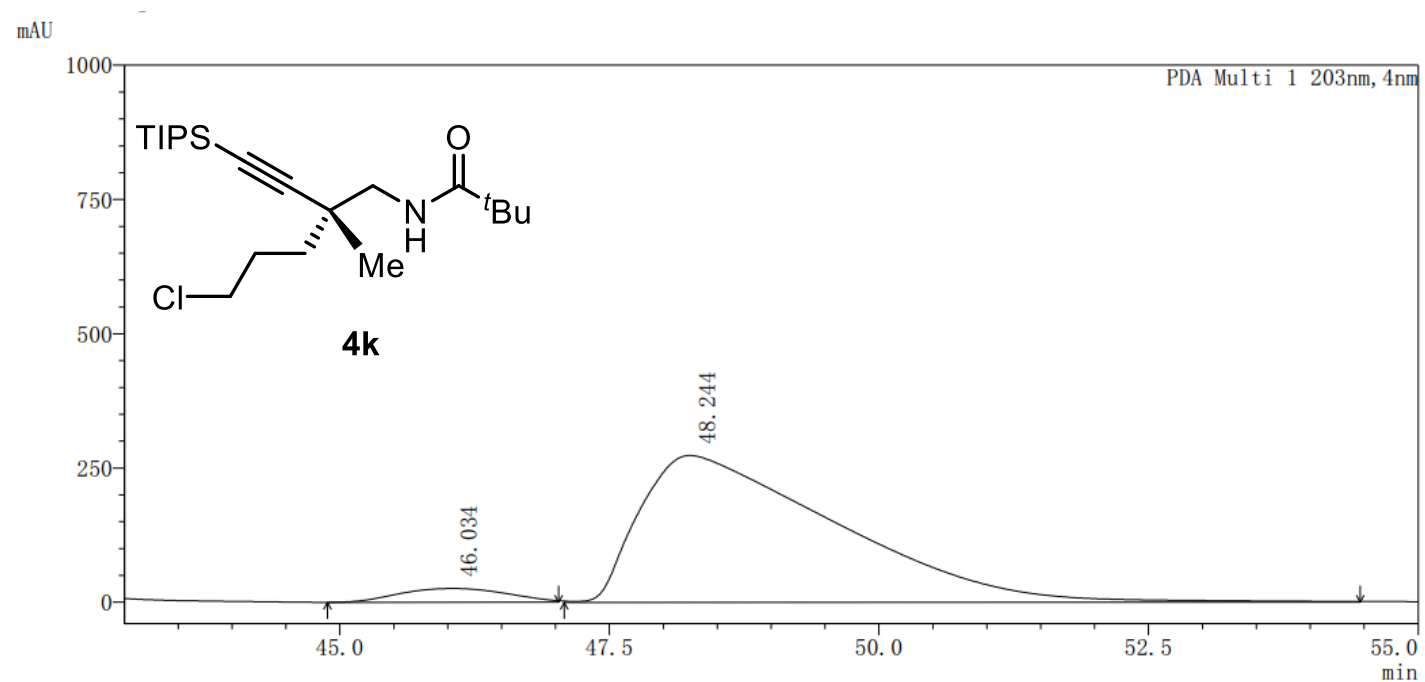

PDA Ch1 203nm

\begin{tabular}{|r|r|r|r|r|}
\hline Peak & Ret. Time & \multicolumn{1}{c|}{ Height } & \multicolumn{1}{c|}{ Area } & \multicolumn{1}{c|}{ Area\% } \\
\hline 1 & 46.034 & 25745 & 1810721 & 4.952 \\
\hline 2 & 48.244 & 273407 & 34757075 & 95.048 \\
\hline
\end{tabular}

Figure S144. HPLC record for chiral 4k 
$\mathrm{mAU}$

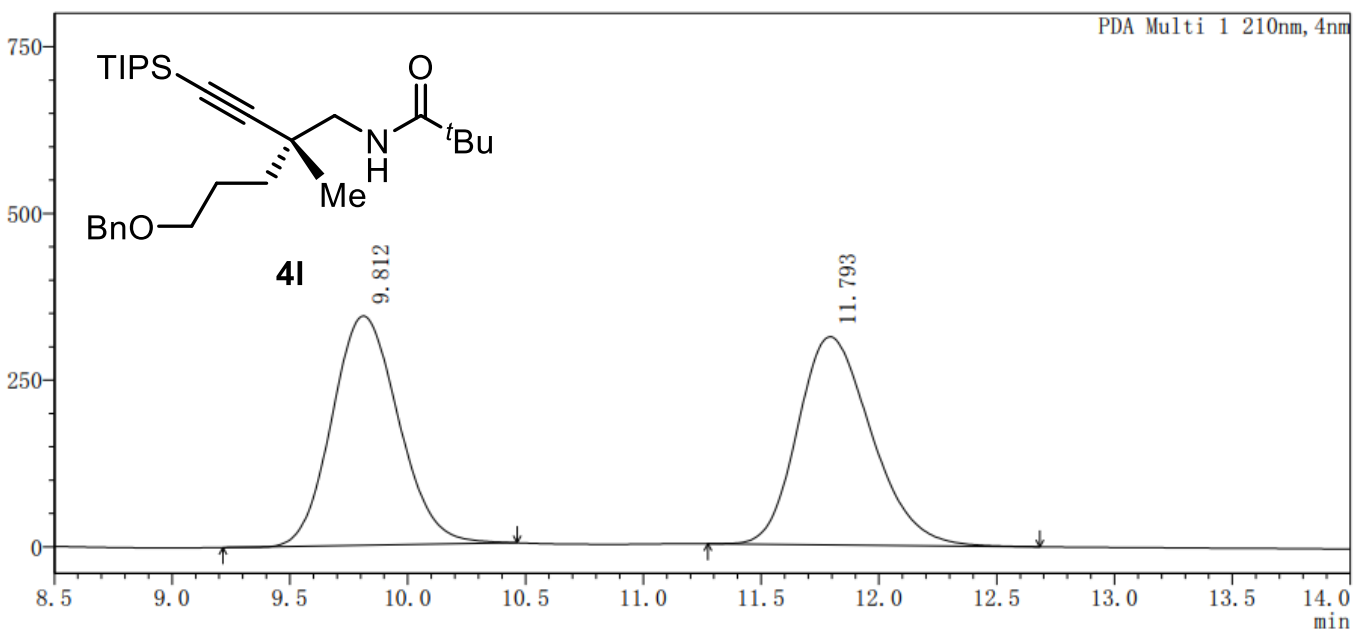

PDA Ch1 210nm

\begin{tabular}{|r|r|r|r|r|}
\hline Peak & Ret. Time & Height & Area & \multicolumn{1}{c|}{ Area\% } \\
\hline 1 & 9.812 & 343989 & 6775334 & 49.853 \\
\hline 2 & 11.793 & 312043 & 6815243 & 50.147 \\
\hline
\end{tabular}

Figure S145. HPLC record for racemic 41

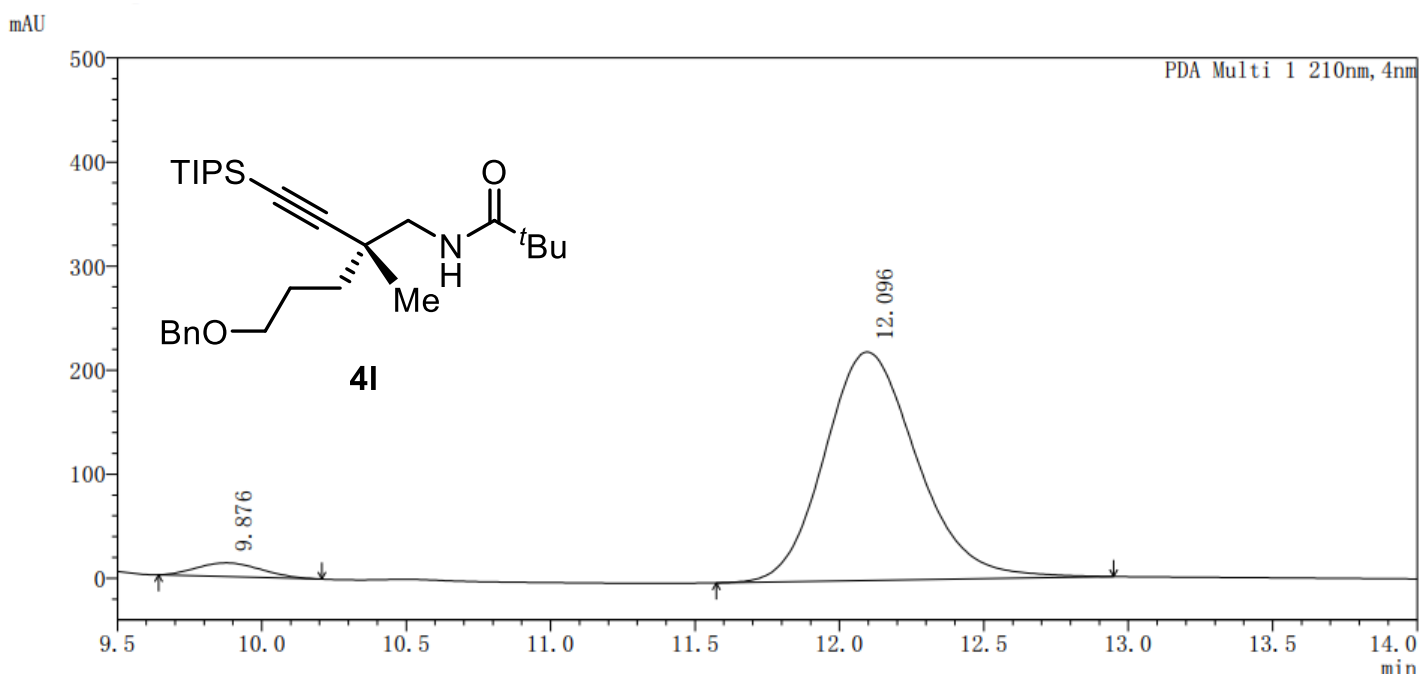

PDA Ch1 210nm
\begin{tabular}{|r|r|r|r|r|}
\hline Peak & Ret. Time & Height & Area & \multicolumn{1}{c|}{ Area $\%$} \\
\hline 1 & 9.876 & 13157 & 204439 & 3.996 \\
\hline 2 & 12.096 & 219632 & 4911637 & 96.004 \\
\hline
\end{tabular}

Figure S146. HPLC record for chiral $4 \mathrm{I}$ 


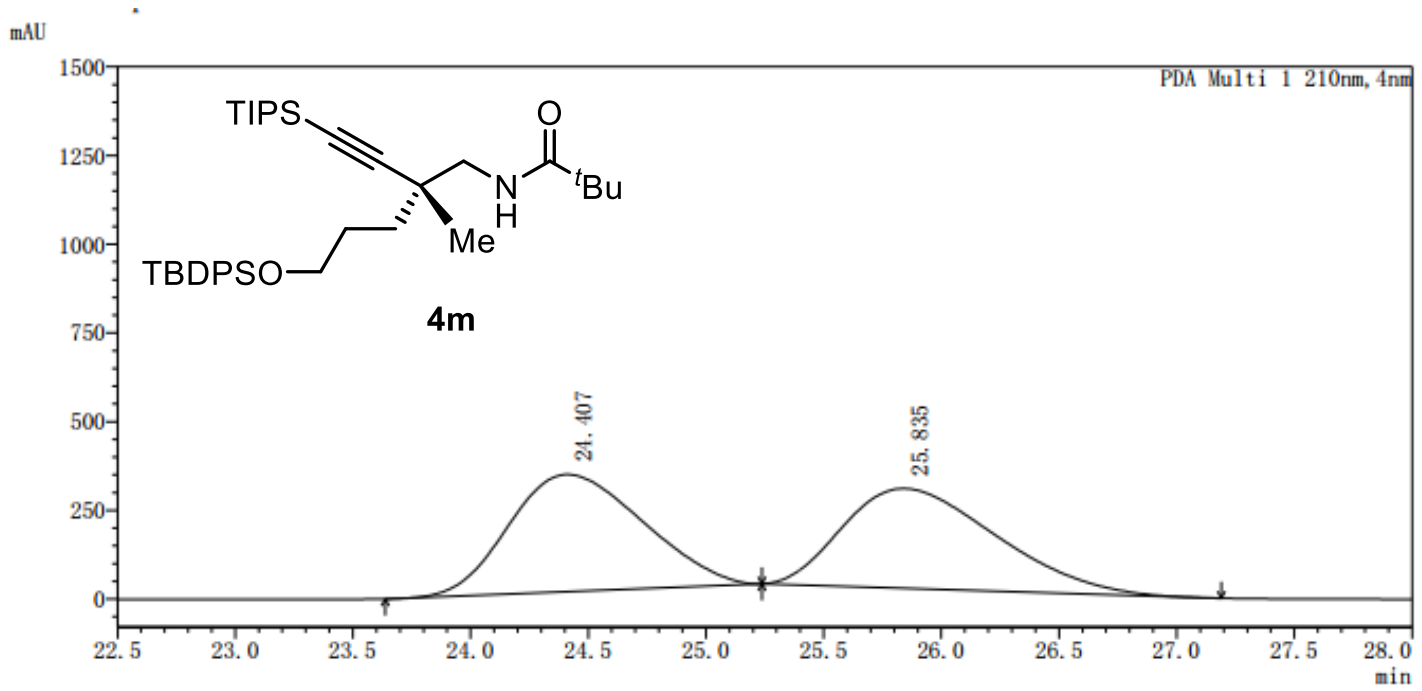

PDA Ch1 210nm
\begin{tabular}{|r|r|r|c|c|}
\hline Peak & Ret. Time & Height & Area & Area\% \\
\hline 1 & 24.407 & 330448 & 13079822 & 50.607 \\
\hline 2 & 25.835 & 281186 & 12766248 & 49.393 \\
\hline
\end{tabular}

Figure S147. HPLC record for racemic 4m

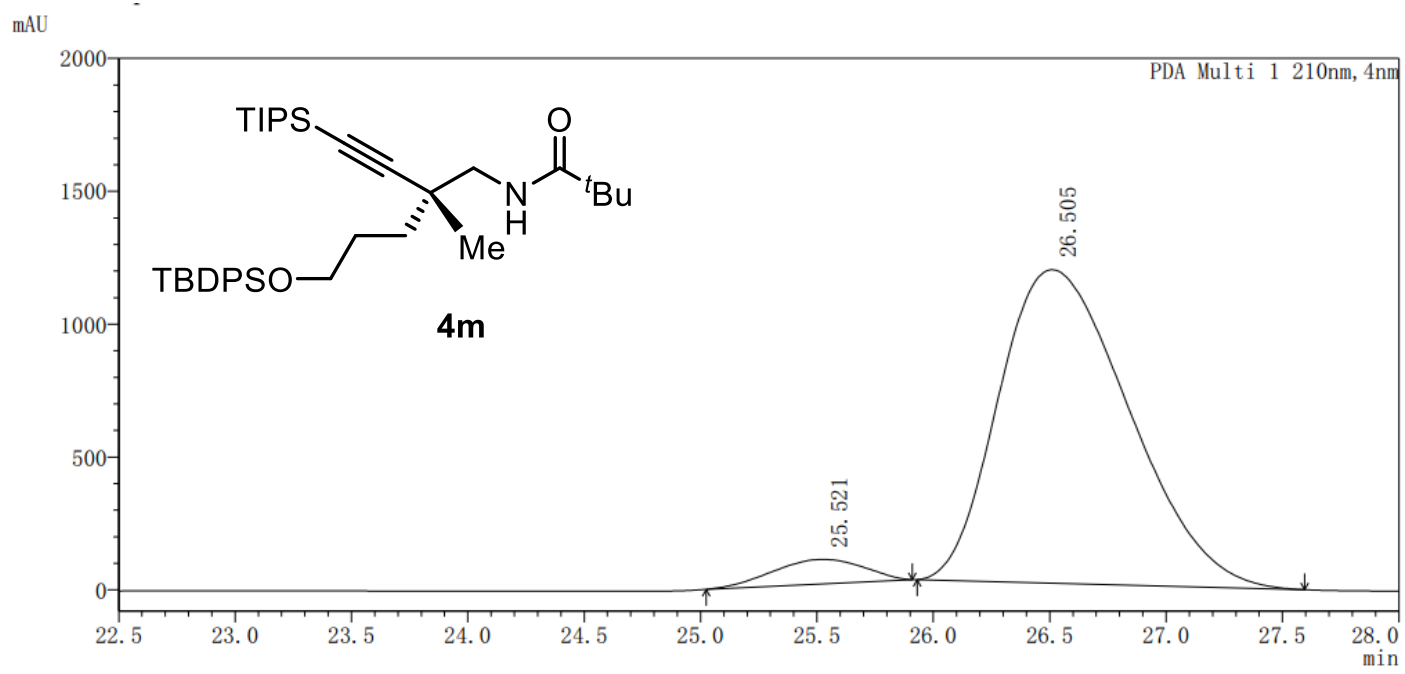

PDA Ch1 210nm
\begin{tabular}{|r|r|r|r|r|}
\hline Peak & Ret. Time & Height & \multicolumn{1}{c|}{ Area } & \multicolumn{1}{|c|}{ Area $\%$} \\
\hline 1 & 25.521 & 92362 & 2519704 & 5.199 \\
\hline 2 & 26.505 & 1179298 & 45944361 & 94.801 \\
\hline
\end{tabular}

Figure S148. HPLC record for chiral 4m 
$\mathrm{mAU}$

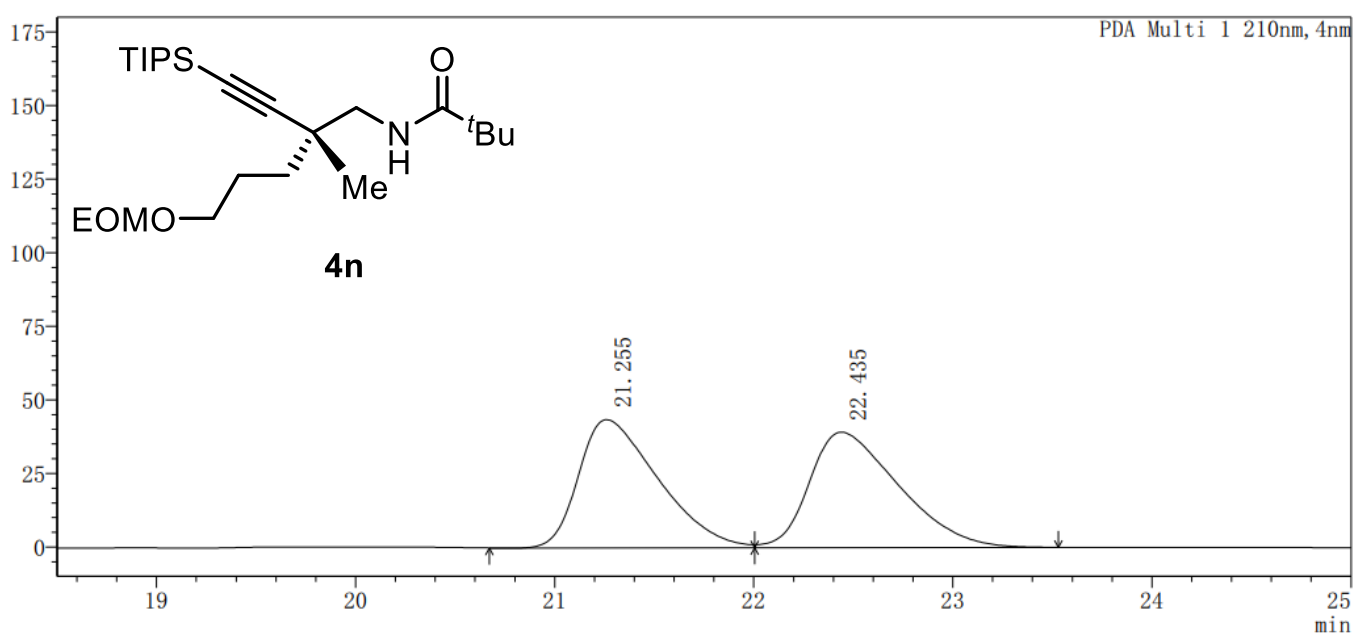

PDA Ch1 210nm

\begin{tabular}{|r|r|r|l|r|}
\hline Peak & Ret. Time & Height & \multicolumn{1}{|c|}{ Area } & \multicolumn{1}{c|}{ Area\% } \\
\hline 1 & 21.255 & 43559 & 1207428 & 49.709 \\
\hline 2 & 22.435 & 39235 & 1221571 & 50.291 \\
\hline
\end{tabular}

Figure S149. HPLC record for racemic 4n

$\mathrm{mAU}$

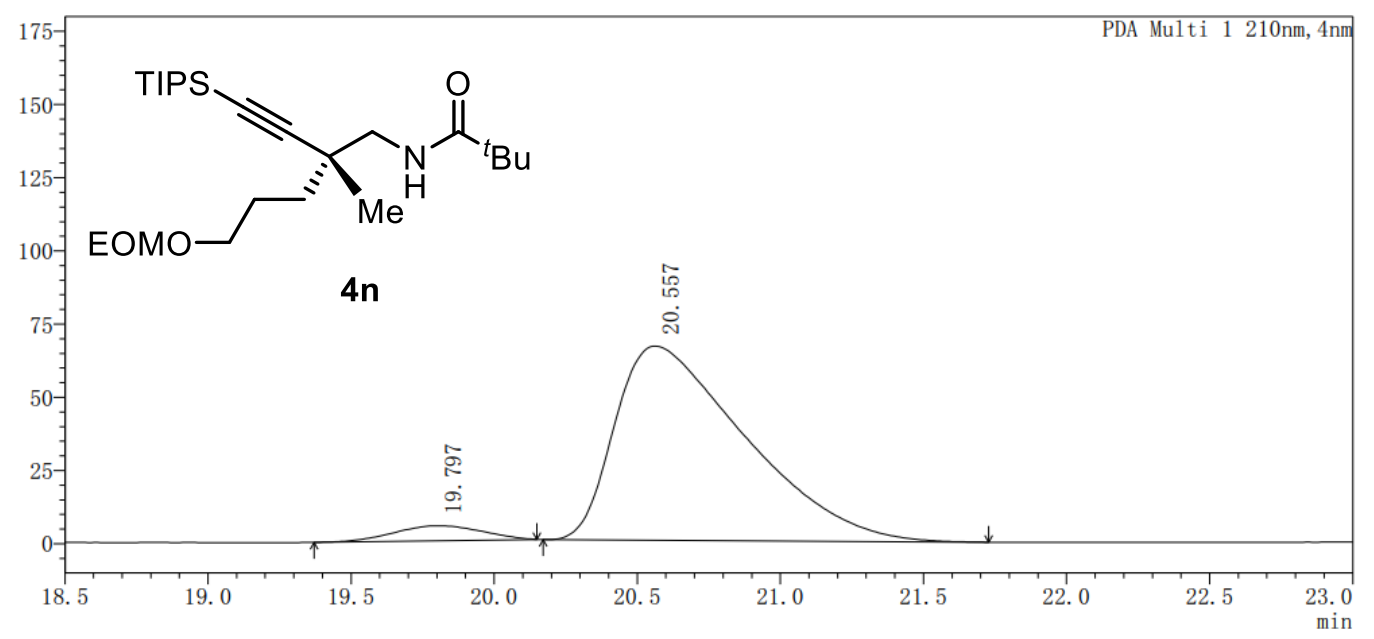

PDA Ch1 210nm

\begin{tabular}{|r|r|r|r|r|}
\hline Peak & Ret. Time & \multicolumn{1}{|c|}{ Height } & \multicolumn{1}{c|}{ Area } & \multicolumn{1}{c|}{ Area\% } \\
\hline 1 & 19.797 & 5122 & 109564 & 4.950 \\
\hline 2 & 20.557 & 66351 & 2103970 & 95.050 \\
\hline
\end{tabular}

Figure S150. HPLC record for chiral 4n 
$\mathrm{mAU}$

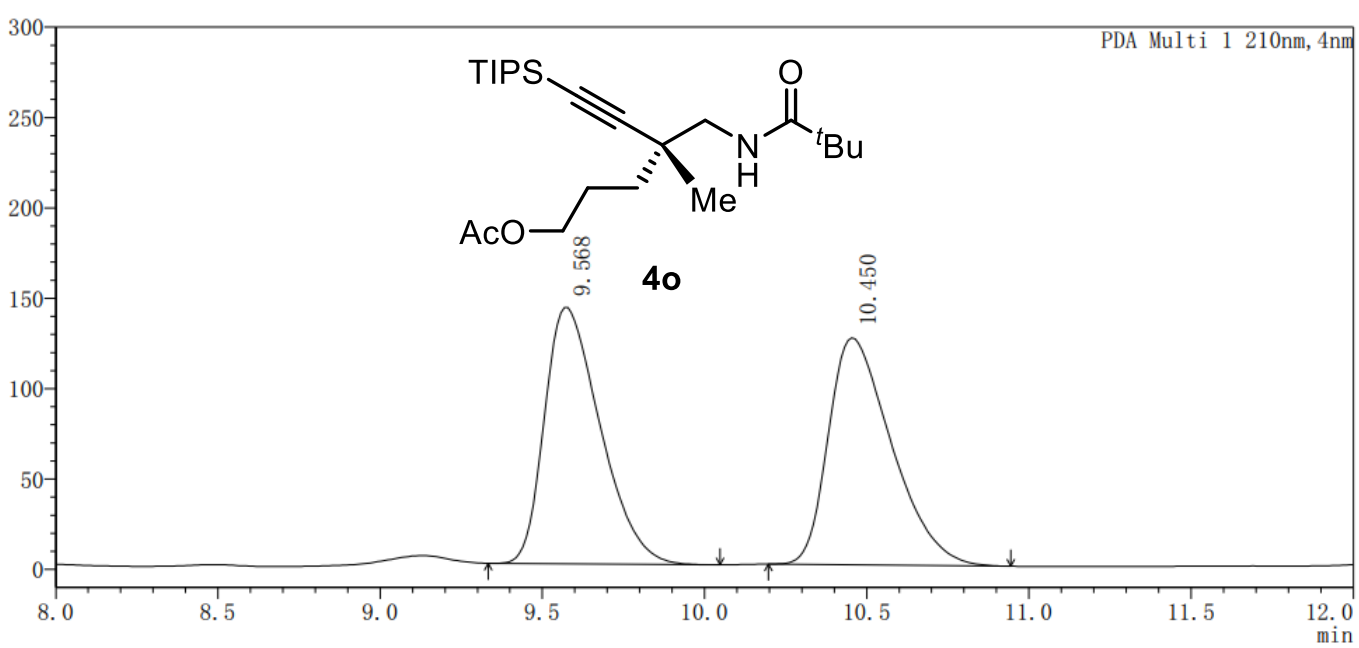

PDA Ch1 210nm

\begin{tabular}{|r|r|r|l|r|}
\hline Peak & Ret. Time & \multicolumn{1}{c|}{ Height } & \multicolumn{1}{l|}{ Area } & \multicolumn{1}{c|}{ Area\% } \\
\hline 1 & 9.568 & 141843 & 1697647 & 49.915 \\
\hline 2 & 10.450 & 125621 & 1703462 & 50.085 \\
\hline
\end{tabular}

Figure S151. HPLC record for racemic 40

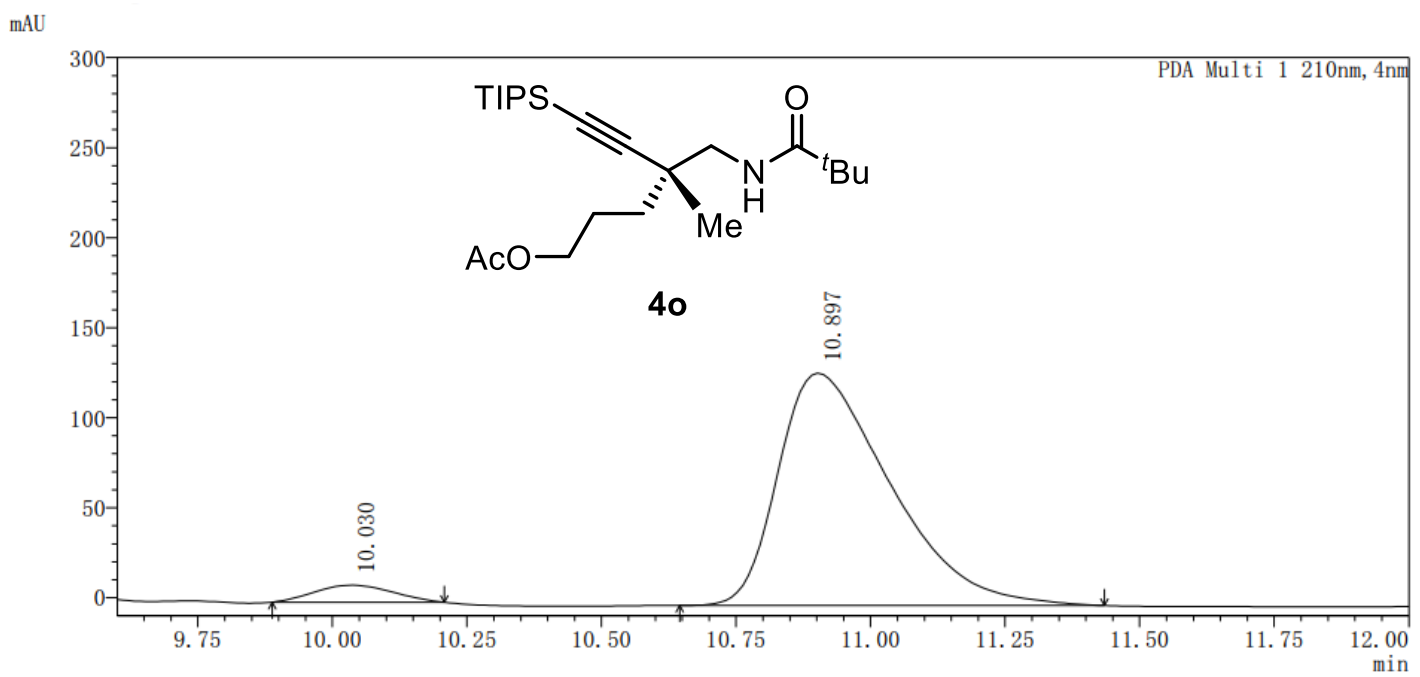

PDA Ch1 210nm
\begin{tabular}{|r|r|r|r|r|}
\hline Peak & Ret. Time & Height & \multicolumn{1}{c|}{ Area } & \multicolumn{1}{c|}{ Area $\%$} \\
\hline 1 & 10.030 & 9507 & 95932 & 4.891 \\
\hline 2 & 10.897 & 129087 & 1865469 & 95.109 \\
\hline
\end{tabular}

Figure S152. HPLC record for chiral 4o 
$\mathrm{mAU}$

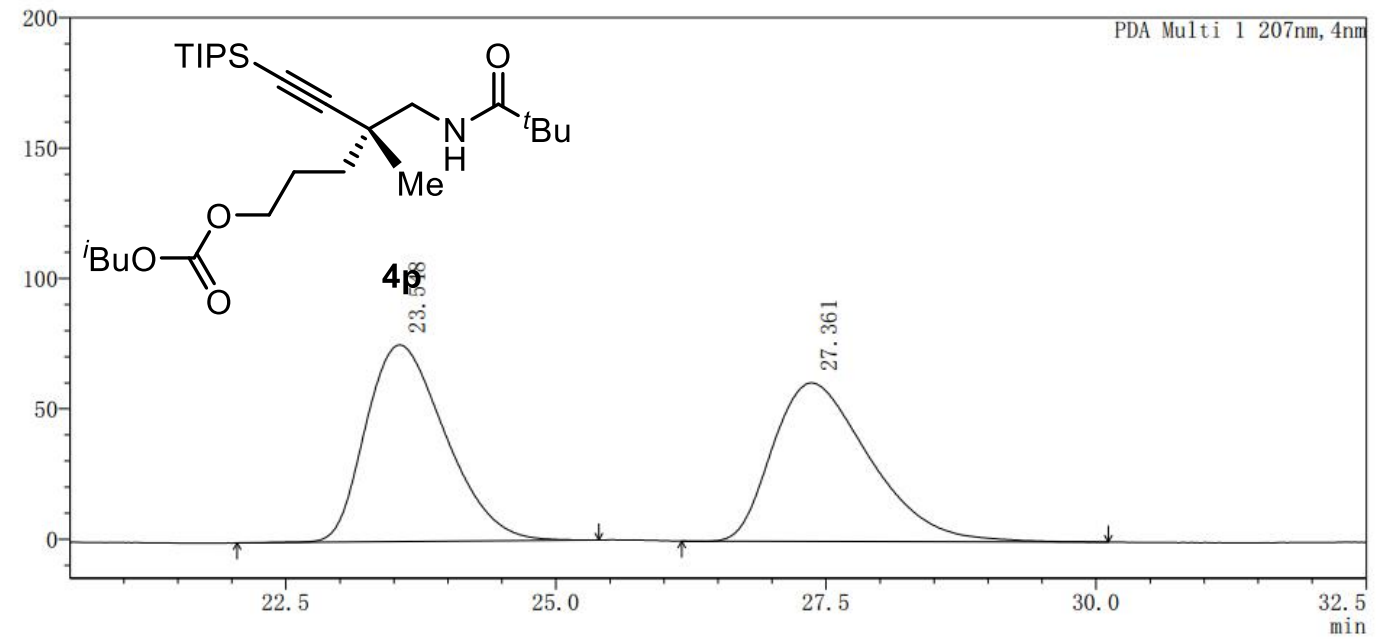

PDA Ch1 207nm

\begin{tabular}{|r|r|r|r|r|}
\hline Peak & Ret. Time & Height & \multicolumn{1}{c|}{ Area } & \multicolumn{1}{c|}{ Area\% } \\
\hline 1 & 23.548 & 75428 & 3865448 & 50.432 \\
\hline 2 & 27.361 & 60802 & 3799231 & 49.568 \\
\hline
\end{tabular}

Figure S153. HPLC record for racemic $4 p$

mAU

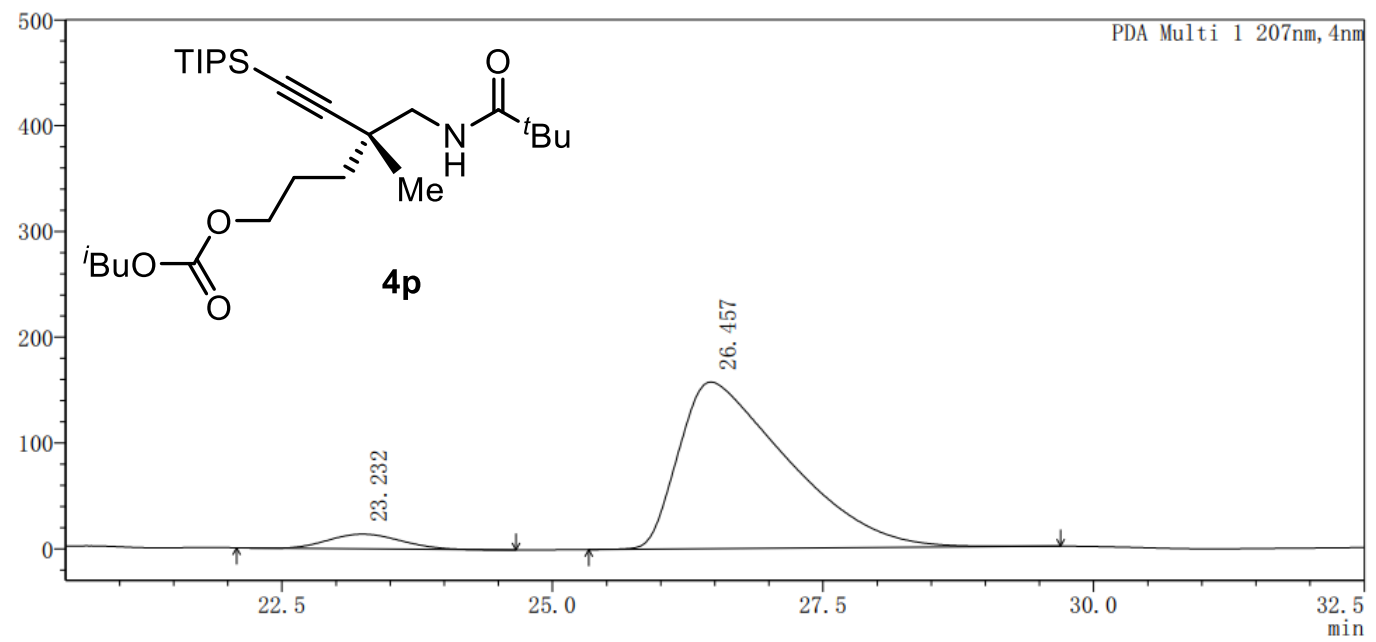

PDA Ch1 207nm

\begin{tabular}{|r|r|r|r|r|}
\hline Peak & Ret. Time & \multicolumn{1}{c|}{ Height } & \multicolumn{1}{c|}{ Area } & \multicolumn{1}{c|}{ Area\% } \\
\hline 1 & 23.232 & 13852 & 640227 & 5.484 \\
\hline 2 & 26.457 & 157437 & 11034294 & 94.516 \\
\hline
\end{tabular}

Figure S154. HPLC record for chiral 4p 
mAU

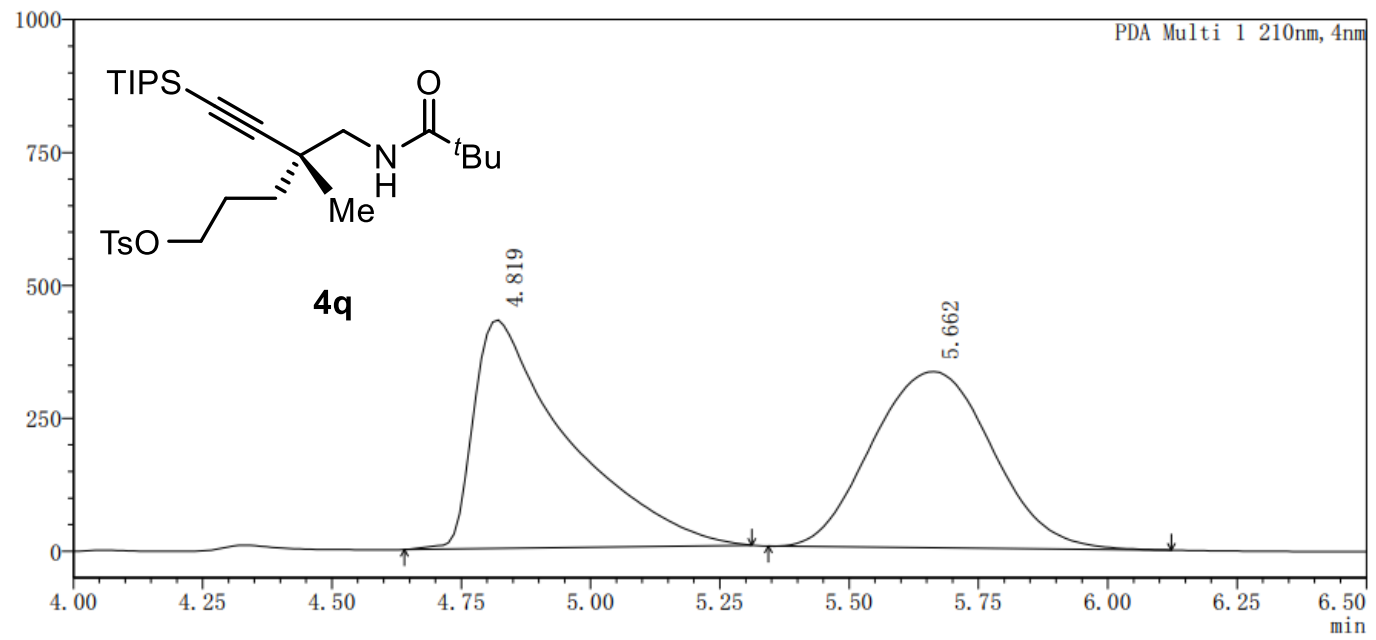

PDA Ch1 210nm

\begin{tabular}{|r|r|r|r|r|}
\hline Peak & Ret. Time & Height & \multicolumn{1}{c|}{ Area } & \multicolumn{1}{c|}{ Area\% } \\
\hline 1 & 4.819 & 429586 & 5445016 & 50.323 \\
\hline 2 & 5.662 & 331663 & 5375085 & 49.677 \\
\hline
\end{tabular}

Figure S155. HPLC record for racemic 4q

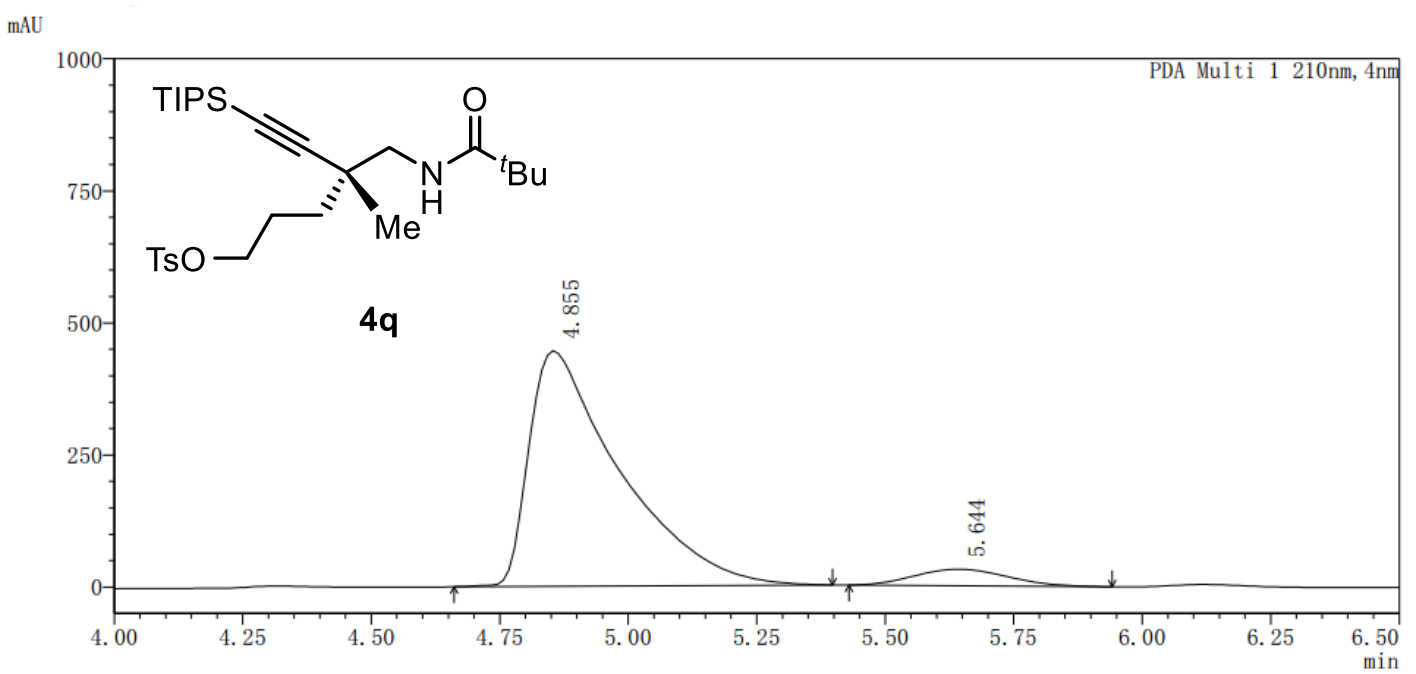

PDA Ch1 210nm

\begin{tabular}{|r|r|r|r|r|}
\hline Peak & Ret. Time & Height & \multicolumn{1}{c|}{ Area } & \multicolumn{1}{c|}{ Area\% } \\
\hline 1 & 4.855 & 445936 & 5458409 & 93.222 \\
\hline 2 & 5.644 & 31412 & 396850 & 6.778 \\
\hline
\end{tabular}

Figure S156. HPLC record for chiral 4q 
$\mathrm{mAU}$

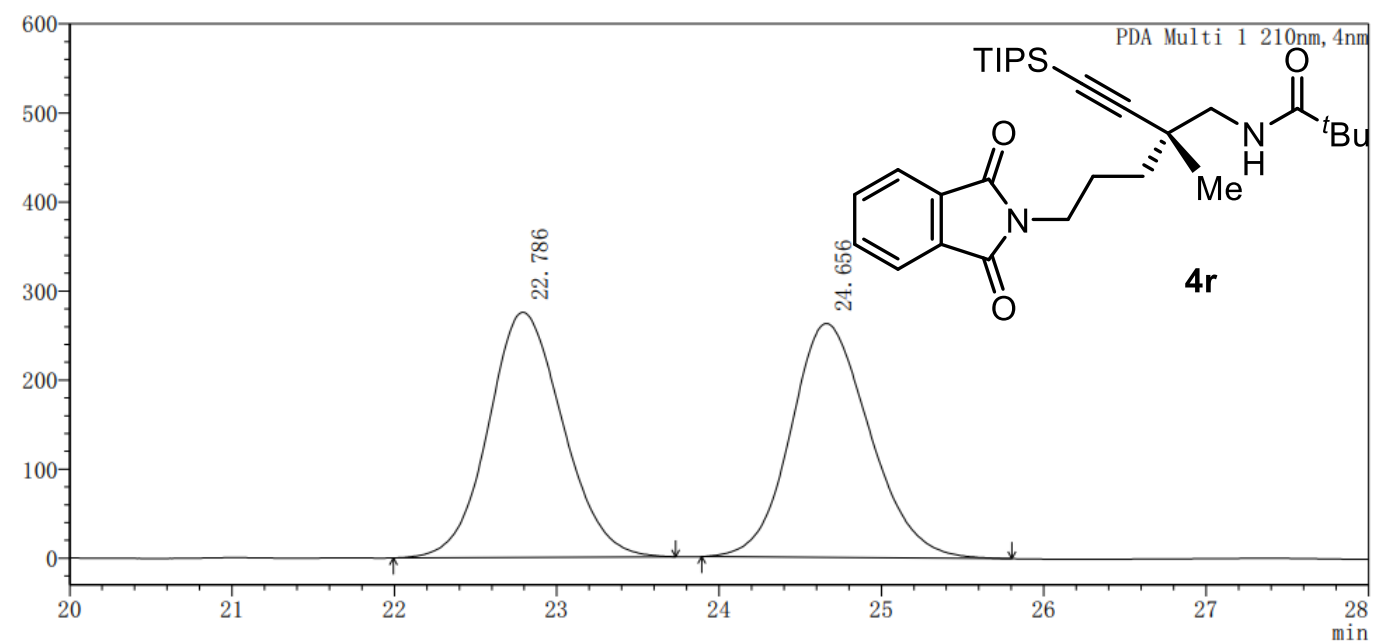

PDA Ch1 210nm

\begin{tabular}{|r|r|r|l|r|}
\hline Peak & Ret. Time & \multicolumn{1}{c|}{ Height } & \multicolumn{1}{l|}{ Area } & \multicolumn{1}{c|}{ Area\% } \\
\hline 1 & 22.786 & 275184 & 8776367 & 49.676 \\
\hline 2 & 24.656 & 262693 & 8890739 & 50.324 \\
\hline
\end{tabular}

Figure S157. HPLC record for racemic 4r

mAU

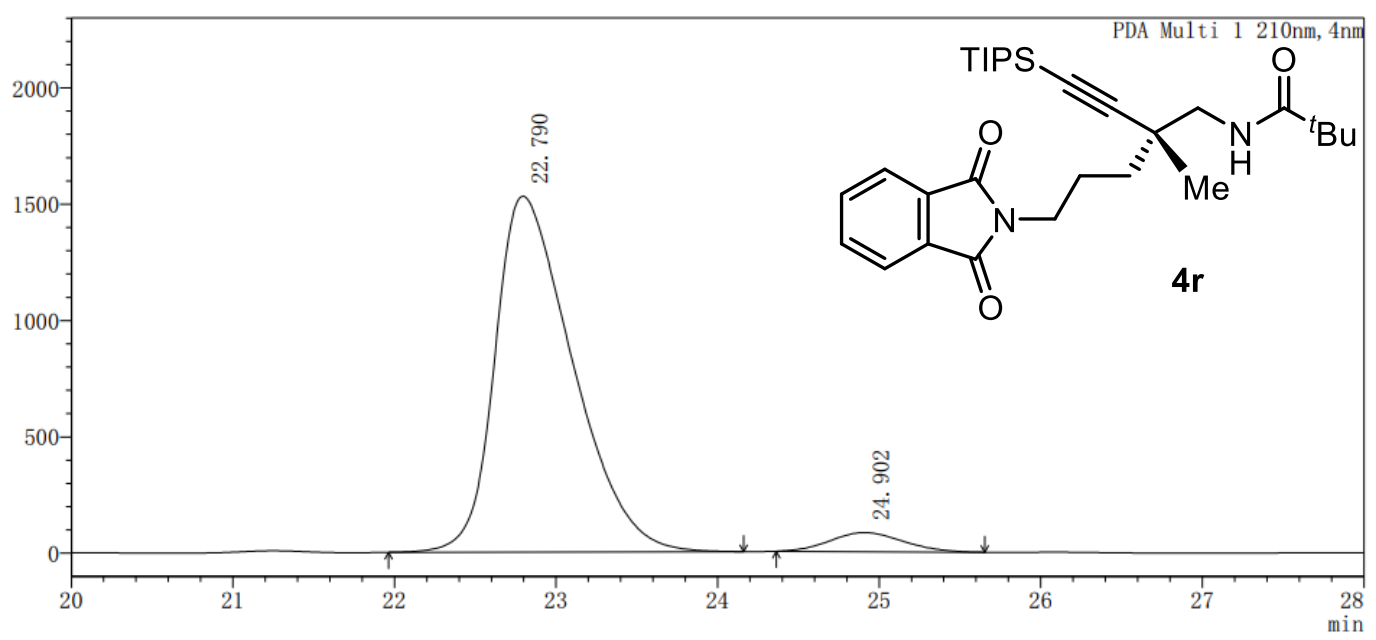

PDA Ch1 210nm

\begin{tabular}{|r|r|r|r|r|}
\hline Peak & Ret. Time & \multicolumn{1}{c|}{ Height } & \multicolumn{1}{c|}{ Area } & \multicolumn{1}{c|}{ Area\% } \\
\hline 1 & 22.790 & 1529124 & 51209947 & 95.210 \\
\hline 2 & 24.902 & 81711 & 2576403 & 4.790 \\
\hline
\end{tabular}

Figure S158. HPLC record for chiral 4r 


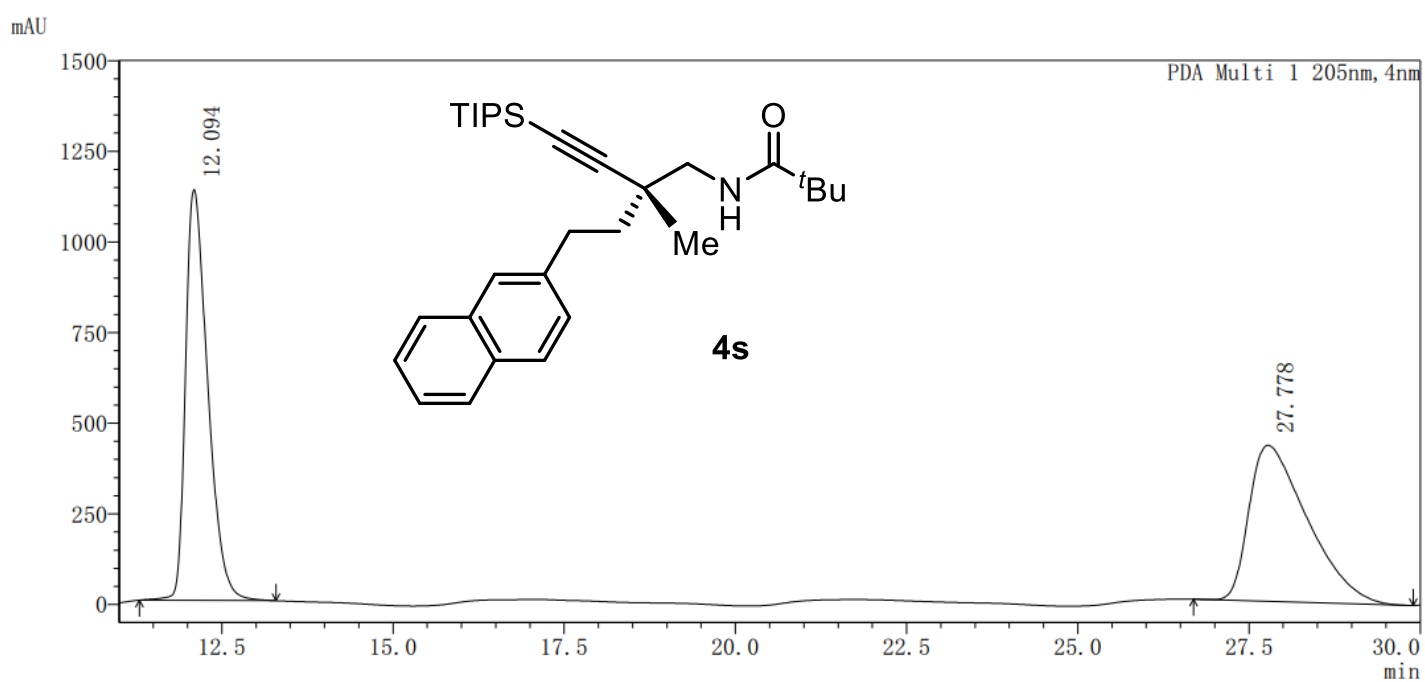

PDA Ch1 205nm

\begin{tabular}{|r|r|r|r|r|}
\hline Peak & Ret. Time & \multicolumn{1}{c|}{ Height } & Area & \multicolumn{1}{c|}{ Area\% } \\
\hline 1 & 12.094 & 1132650 & 25604239 & 50.112 \\
\hline 2 & 27.778 & 430188 & 25489900 & 49.888 \\
\hline
\end{tabular}

Figure S159. HPLC record for racemic 4s

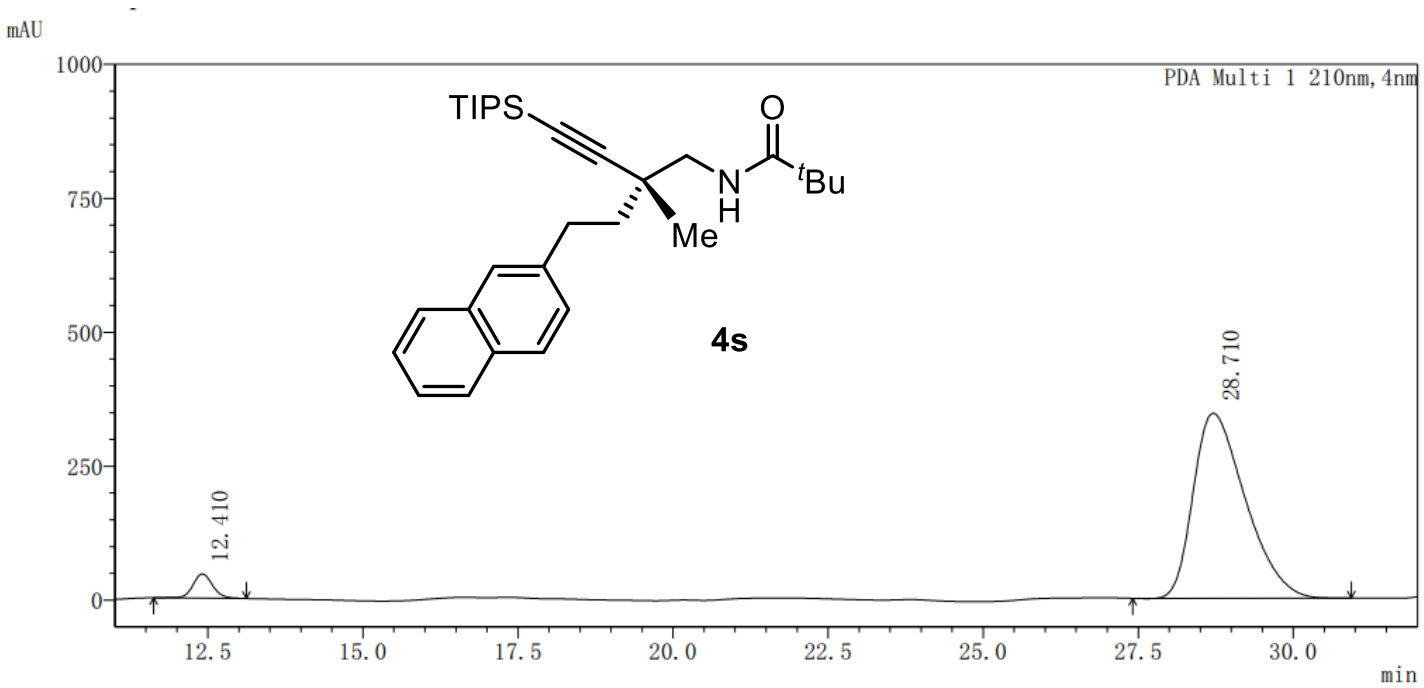

PDA Ch1 210nm

\begin{tabular}{|r|r|r|r|r|}
\hline Peak & Ret. Time & \multicolumn{1}{c|}{ Height } & \multicolumn{1}{c|}{ Area } & \multicolumn{1}{c|}{ Area\% } \\
\hline 1 & 12.410 & 45321 & 1001343 & 4.729 \\
\hline 2 & 28.710 & 345320 & 20174199 & 95.271 \\
\hline
\end{tabular}

Figure S160. HPLC record for chiral 4s 


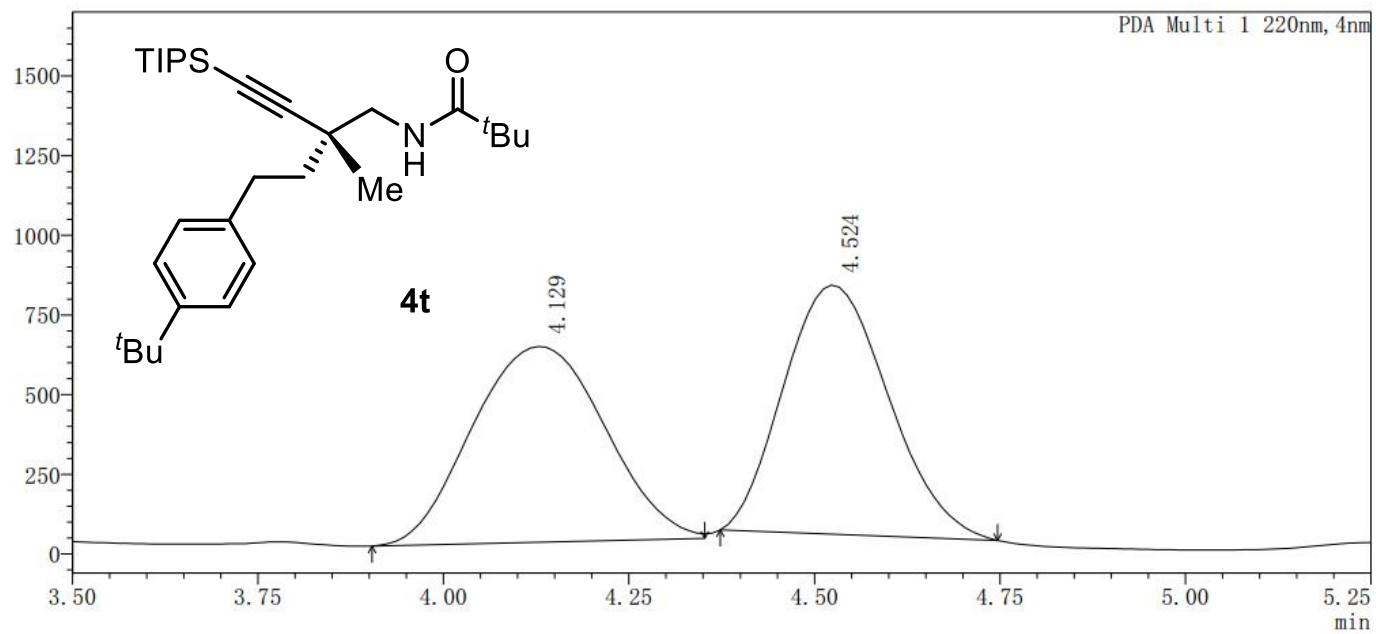

PDA Ch1 220nm

\begin{tabular}{|r|r|r|l|r|}
\hline Peak & Ret. Time & \multicolumn{1}{c|}{ Height } & \multicolumn{1}{l|}{ Area } & \multicolumn{1}{c|}{ Area\% } \\
\hline 1 & 4.129 & 614474 & 7648812 & 49.882 \\
\hline 2 & 4.524 & 781811 & 7685043 & 50.118 \\
\hline
\end{tabular}

Figure S161. HPLC record for racemic 4t

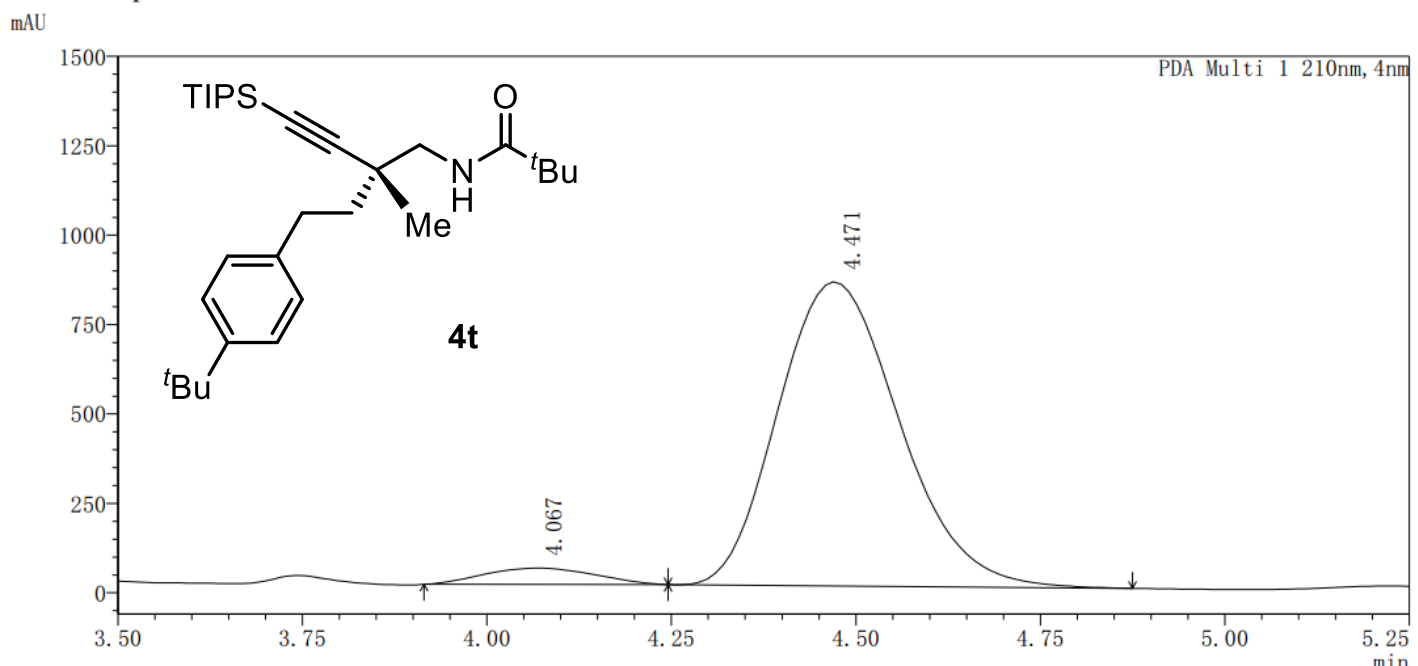

PDA Ch1 210nm
\begin{tabular}{|r|r|r|r|r|}
\hline Peak & Ret. Time & Height & \multicolumn{1}{c|}{ Area } & \multicolumn{1}{c|}{ Area $\%$} \\
\hline 1 & 4.067 & 45772 & 479842 & 4.699 \\
\hline 2 & 4.471 & 850281 & 9731181 & 95.301 \\
\hline
\end{tabular}

Figure S162. HPLC record for chiral 4t 


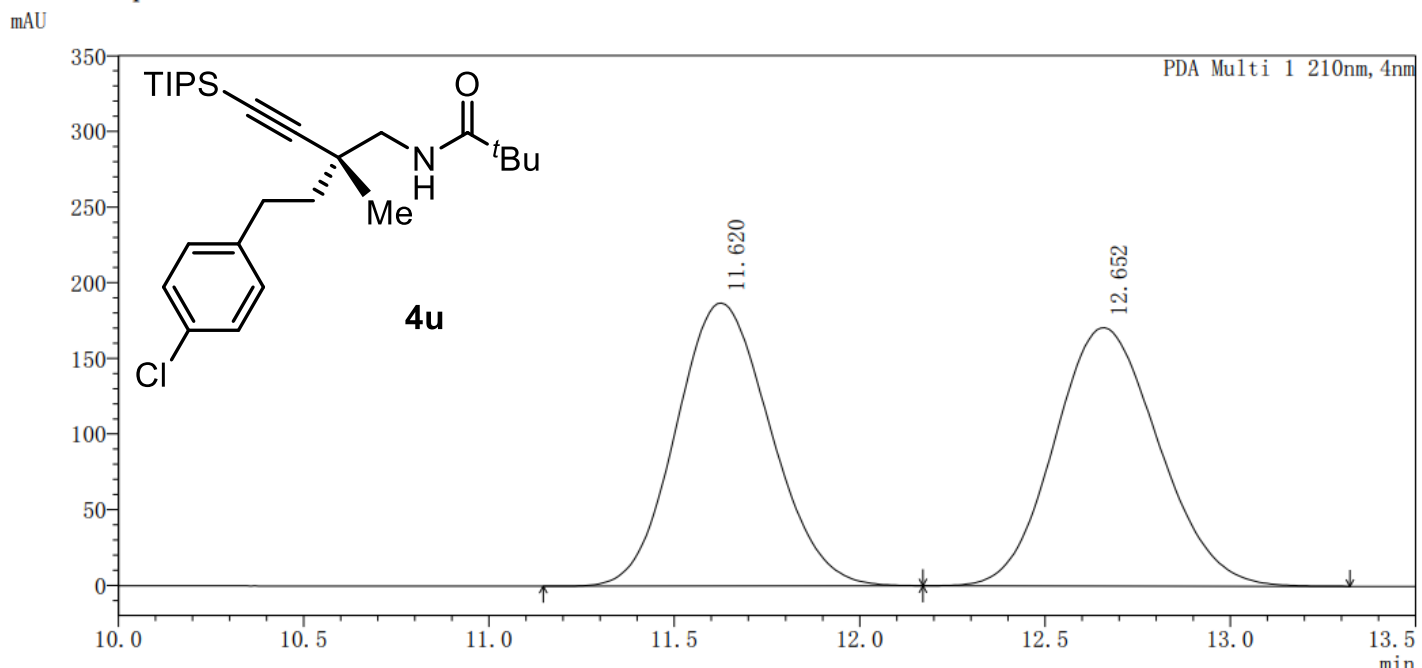

PDA Ch1 210nm
\begin{tabular}{|r|r|r|l|r|}
\hline Peak & Ret. Time & Height & \multicolumn{1}{|c|}{ Area } & \multicolumn{1}{c|}{ Area $\%$} \\
\hline 1 & 11.620 & 187034 & 3315497 & 49.816 \\
\hline 2 & 12.652 & 170609 & 3339957 & 50.184 \\
\hline
\end{tabular}

Figure S163. HPLC record for racemic 4u

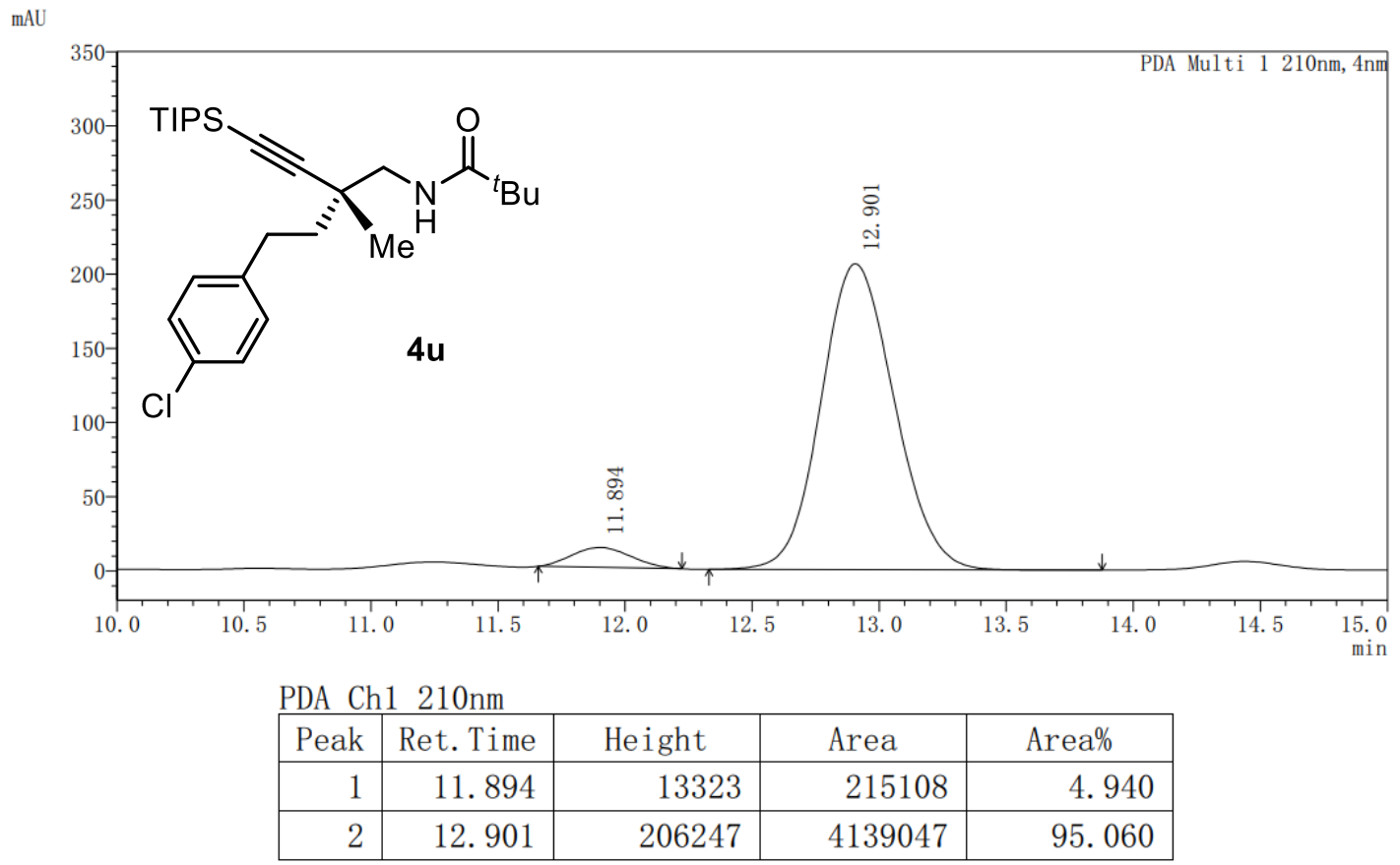

Figure S164. HPLC record for chiral 4u 
mAU

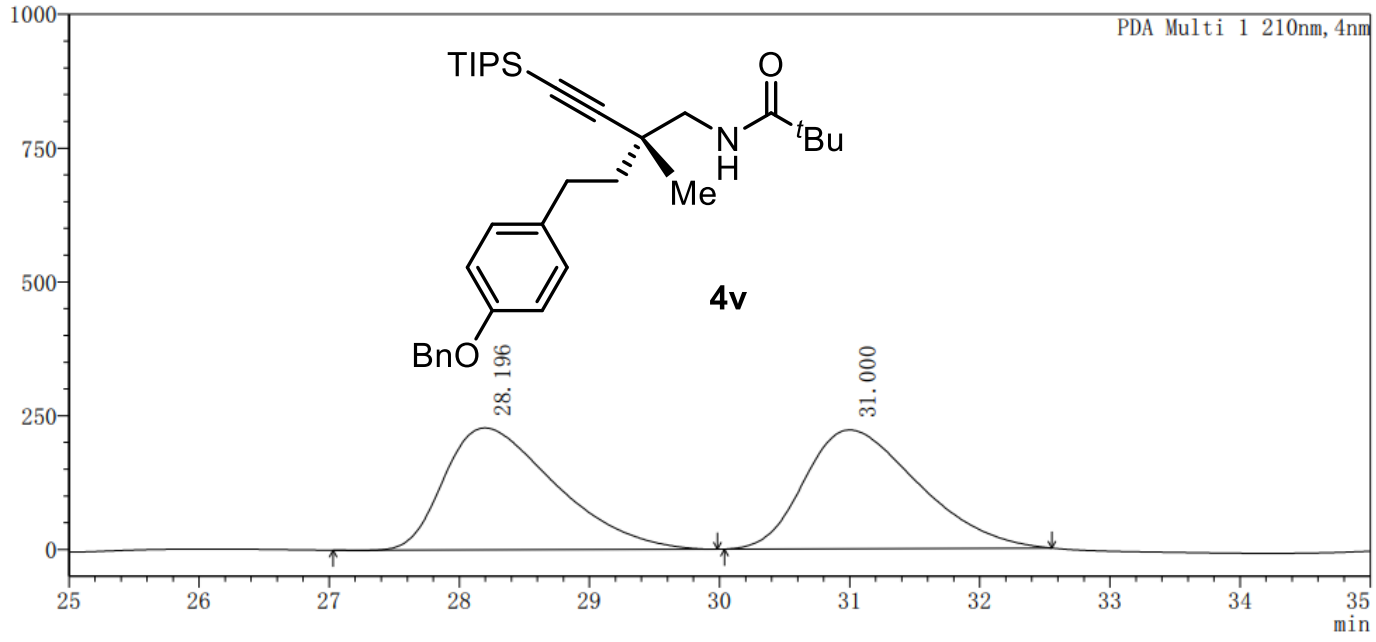

PDA Ch1 210nm

\begin{tabular}{|r|r|r|r|r|}
\hline Peak & Ret. Time & \multicolumn{1}{c|}{ Height } & \multicolumn{1}{c|}{ Area } & \multicolumn{1}{c|}{ Area\% } \\
\hline 1 & 28.196 & 227859 & 13597508 & 50.061 \\
\hline 2 & 31.000 & 222056 & 13564111 & 49.939 \\
\hline
\end{tabular}

Figure S165. HPLC record for racemic 4v

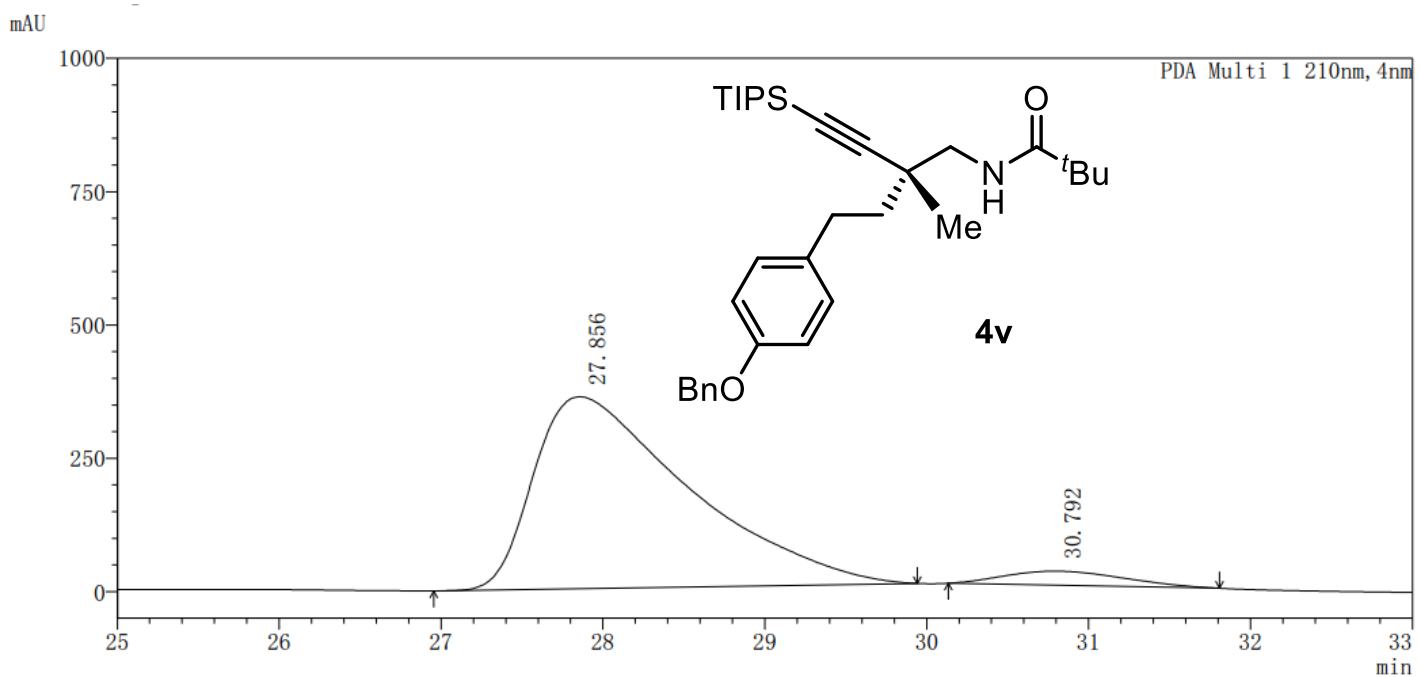

PDA Ch1 210nm

\begin{tabular}{|r|r|r|r|r|}
\hline Peak & Ret. Time & \multicolumn{1}{c|}{ Height } & \multicolumn{1}{c|}{ Area } & \multicolumn{1}{c|}{ Area\% } \\
\hline 1 & 27.856 & 359890 & 23965434 & 94.637 \\
\hline 2 & 30.792 & 26393 & 1358002 & 5.363 \\
\hline
\end{tabular}

Figure S166. HPLC record for chiral 4v 


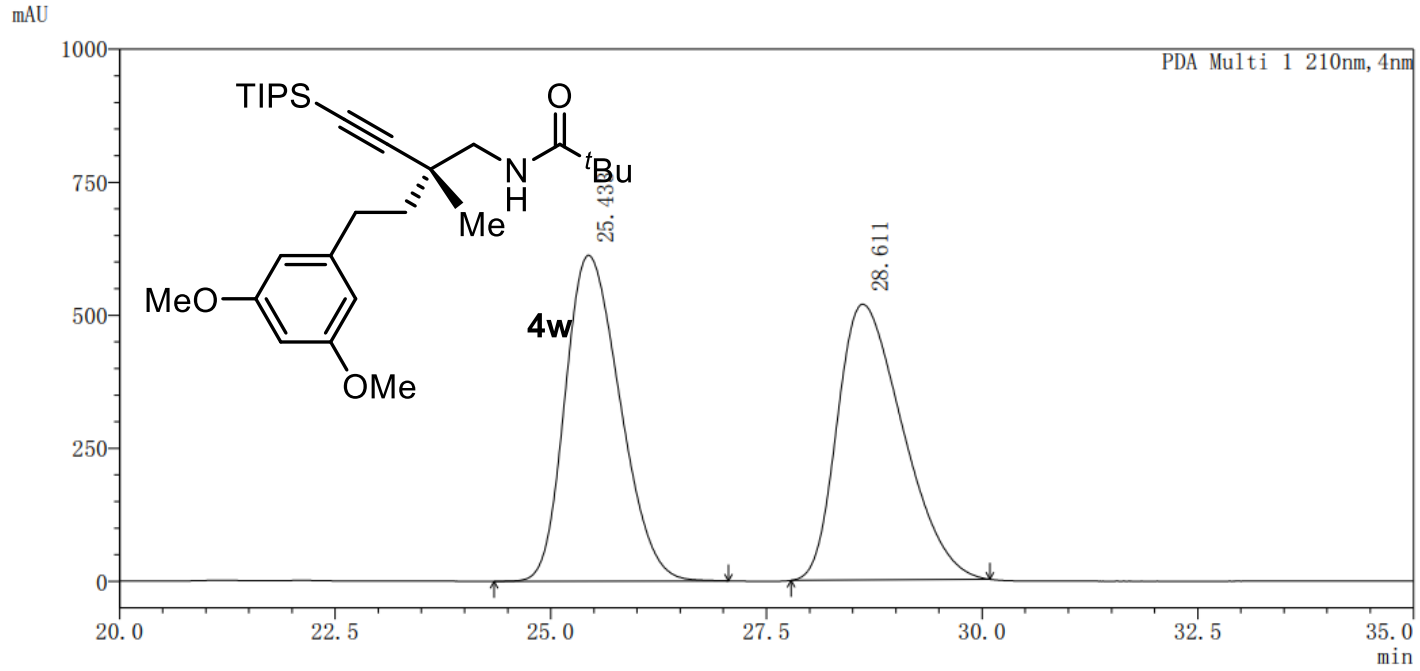

PDA Ch1 210nm

\begin{tabular}{|r|r|r|r|r|}
\hline Peak & Ret. Time & Height & Area & \multicolumn{1}{c|}{ Area\% } \\
\hline 1 & 25.433 & 612460 & 27104153 & 49.762 \\
\hline 2 & 28.611 & 518441 & 27363924 & 50.238 \\
\hline
\end{tabular}

Figure S167. HPLC record for racemic 4w

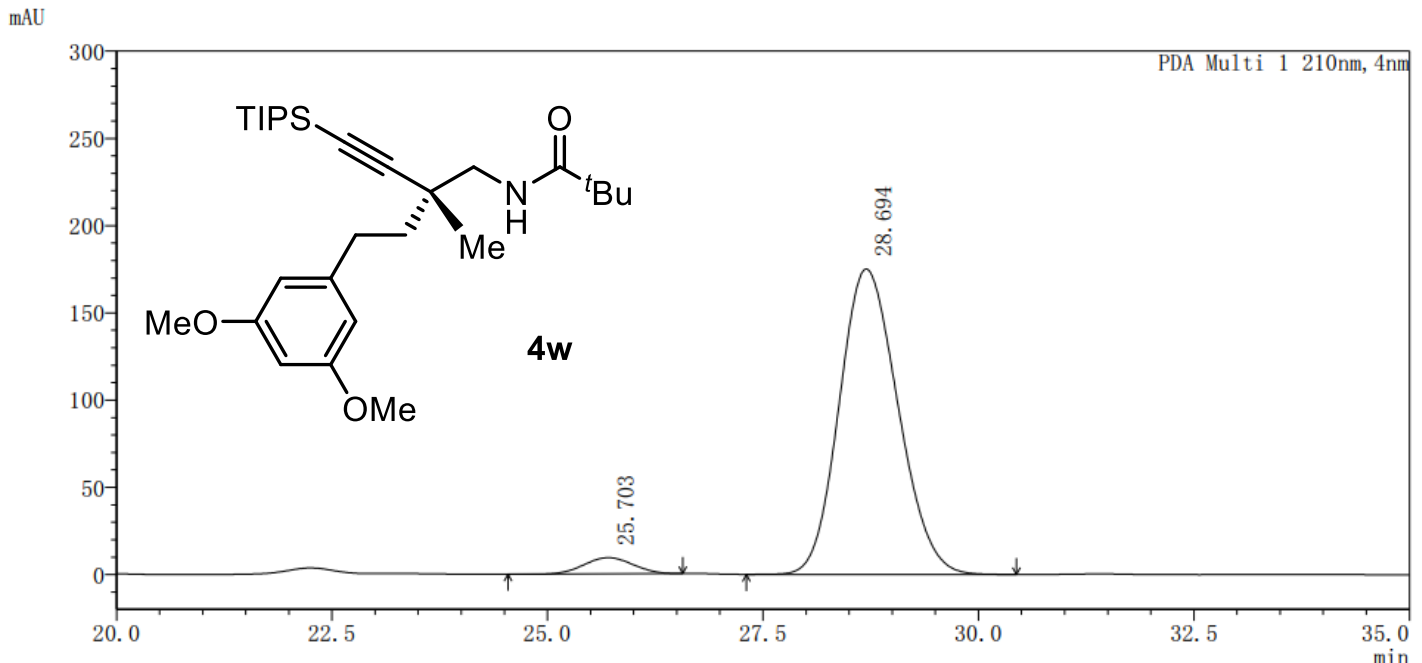

PDA Ch1 210nm
\begin{tabular}{|r|r|r|r|r|}
\hline Peak & Ret. Time & Height & Area & \multicolumn{1}{c|}{ Area $\%$} \\
\hline 1 & 25.703 & 9249 & 363131 & 4.188 \\
\hline 2 & 28.694 & 174975 & 8307568 & 95.812 \\
\hline
\end{tabular}

Figure S168. HPLC record for chiral 4w 
$\mathrm{mAU}$

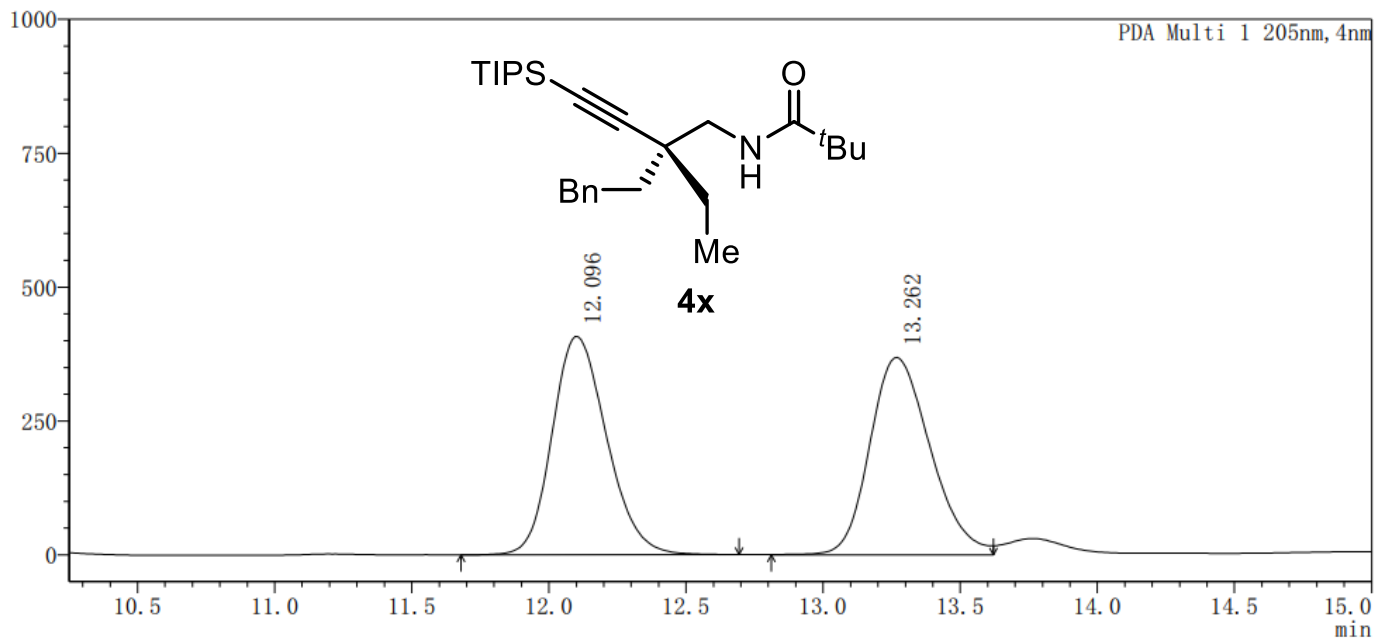

PDA Ch1 205nm

\begin{tabular}{|r|r|r|r|r|}
\hline Peak & Ret. Time & Height & \multicolumn{1}{c|}{ Area } & \multicolumn{1}{c|}{ Area\% } \\
\hline 1 & 12.096 & 407468 & 5647167 & 49.889 \\
\hline 2 & 13.262 & 368858 & 5672307 & 50.111 \\
\hline
\end{tabular}

Figure S169. HPLC record for racemic 4x

$\mathrm{mAU}$

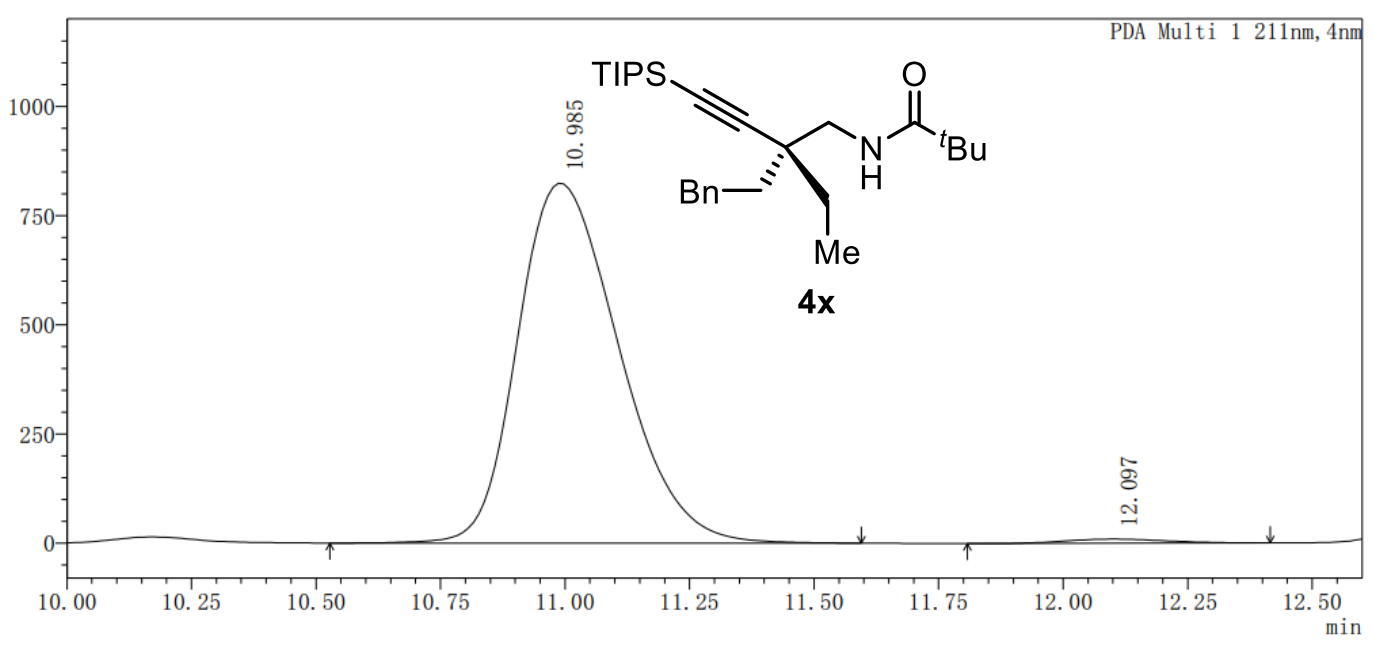

PDA Ch1 211nm

\begin{tabular}{|r|r|r|r|r|}
\hline Peak & Ret. Time & \multicolumn{1}{c|}{ Height } & \multicolumn{1}{c|}{ Area } & \multicolumn{1}{c|}{ Area\% } \\
\hline 1 & 10.985 & 823814 & 11662751 & 98.860 \\
\hline 2 & 12.097 & 9583 & 134498 & 1.140 \\
\hline
\end{tabular}

Figure S170. HPLC record for chiral 4x 


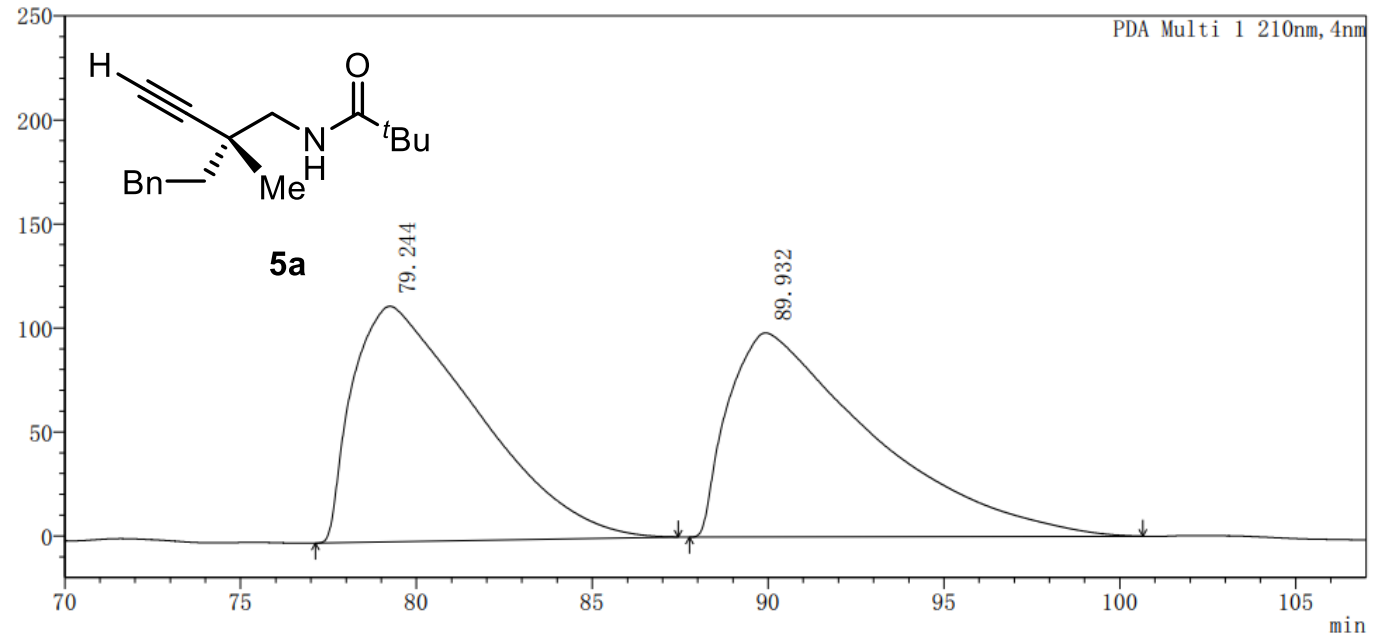

PDA Ch1 210nm

\begin{tabular}{|r|r|r|r|r|}
\hline Peak & Ret. Time & Height & \multicolumn{1}{c|}{ Area } & \multicolumn{1}{c|}{ Area\% } \\
\hline 1 & 79.244 & 113273 & 27860406 & 49.992 \\
\hline 2 & 89.932 & 98101 & 27869459 & 50.008 \\
\hline
\end{tabular}

Figure S171. HPLC record for racemic 5a

mAU

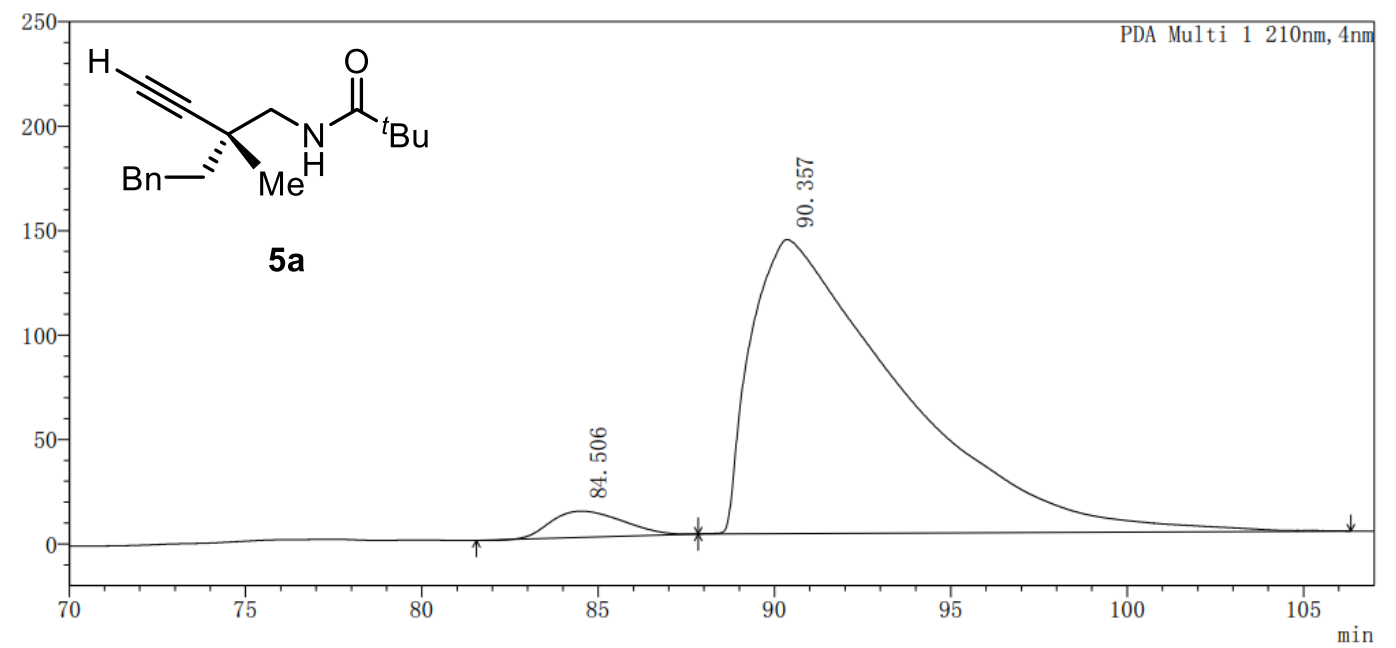

PDA Ch1 210nm

\begin{tabular}{|r|r|r|r|r|}
\hline Peak & Ret. Time & \multicolumn{1}{c|}{ Height } & \multicolumn{1}{c|}{ Area } & \multicolumn{1}{c|}{ Area\% } \\
\hline 1 & 84.506 & 12571 & 1782011 & 4.109 \\
\hline 2 & 90.357 & 140734 & 41586119 & 95.891 \\
\hline
\end{tabular}

Figure S172. HPLC record for chiral 5a 
mAU

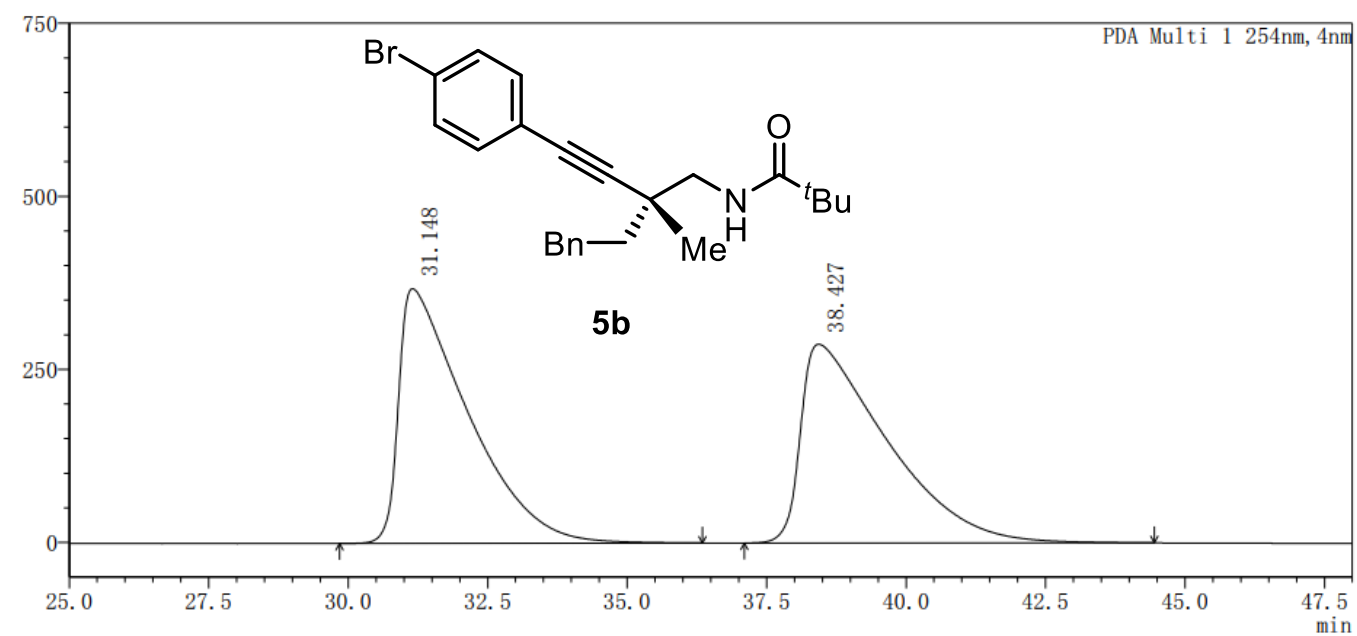

PDA Ch1 254nm

\begin{tabular}{|r|r|r|r|r|r|}
\hline 峰号 & 保留时间 & 峰宽(高度 50\%) & 高度 & 面积 & 面积\% \\
\hline 1 & 31.148 & 1.292 & 367864 & 31823210 & 50.719 \\
\hline 2 & 38.427 & 1.616 & 287141 & 30921268 & 49.281 \\
\hline
\end{tabular}

Figure S173. HPLC record for racemic 5b

mAU

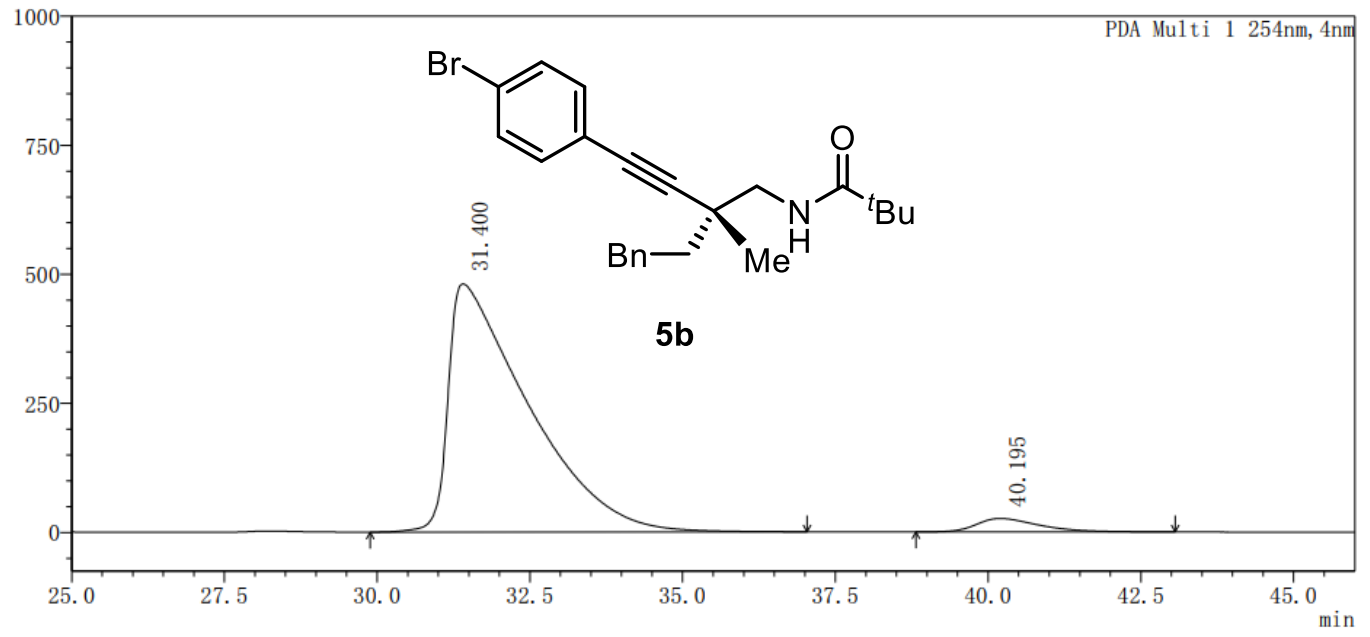

PDA Ch1 254nm

\begin{tabular}{|r|r|r|r|r|r|}
\hline 峰号 & 保留时间 & 峰宽 (高度 50\%) & 高度 & \multicolumn{1}{c|}{ 面积 } & \multicolumn{1}{c|}{ 面积\% } \\
\hline 1 & 31.400 & 1.359 & 480739 & 43853362 & 95.883 \\
\hline 2 & 40.195 & 1.072 & 26129 & 1883004 & 4.117 \\
\hline
\end{tabular}

Figure S174. HPLC record for chiral $\mathbf{5 b}$ 


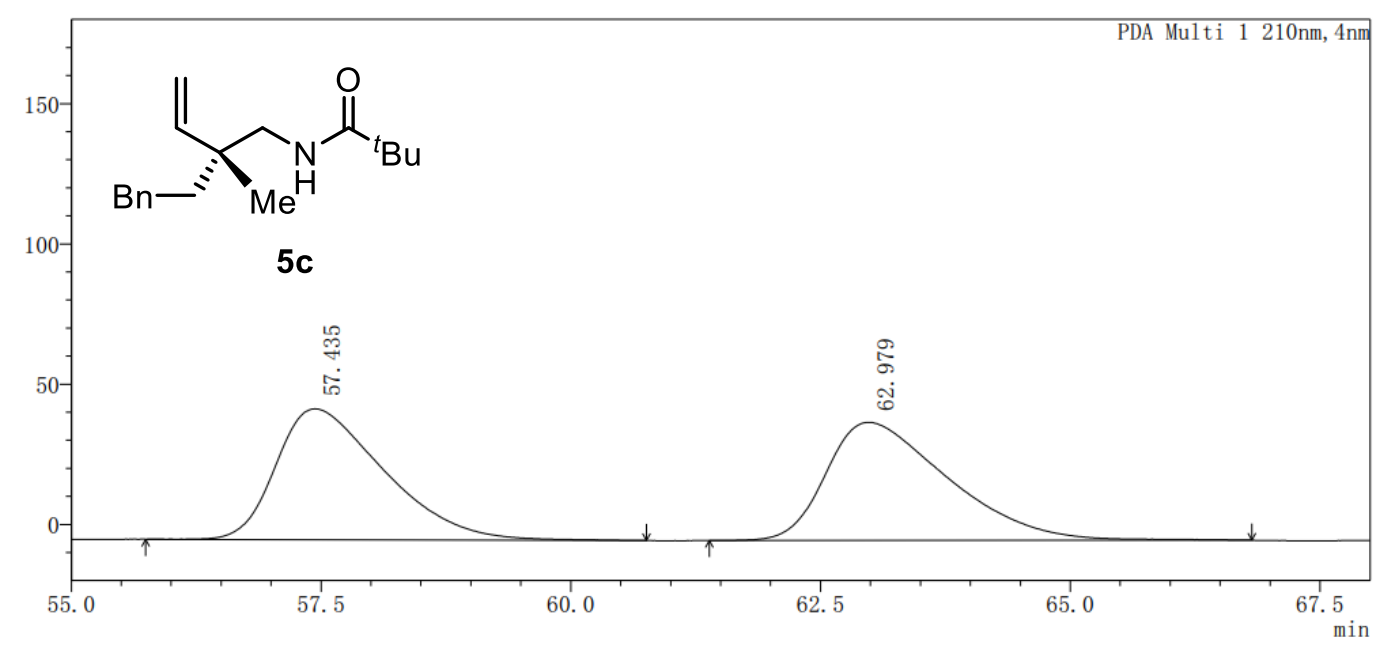

PDA Ch1 210nm
\begin{tabular}{|r|r|r|l|r|}
\hline Peak & Ret. Time & Height & \multicolumn{1}{|l|}{ Area } & \multicolumn{1}{c|}{ Area $\%$} \\
\hline 1 & 57.435 & 46649 & 3519154 & 49.584 \\
\hline 2 & 62.979 & 42094 & 3578207 & 50.416 \\
\hline
\end{tabular}

Figure S175. HPLC record for racemic 5c

mAU

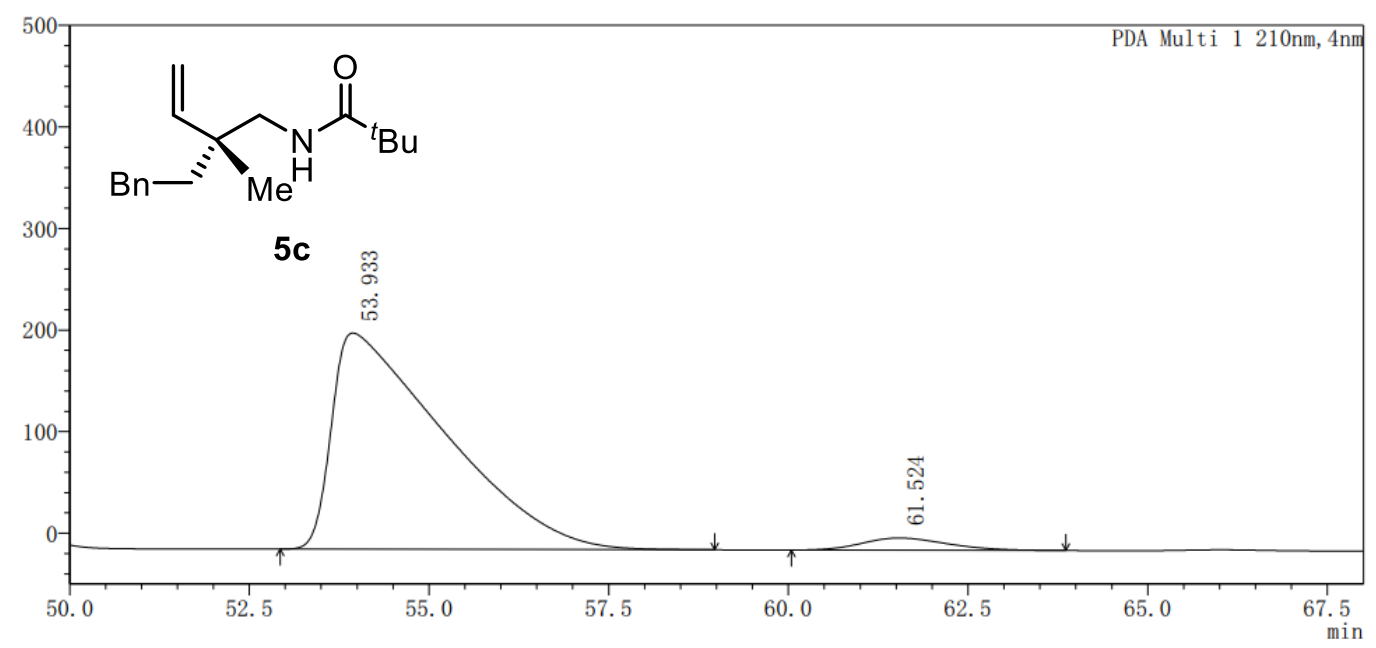

PDA Ch1 210nm

\begin{tabular}{|r|r|r|r|r|}
\hline Peak & Ret. Time & Height & \multicolumn{1}{c|}{ Area } & \multicolumn{1}{c|}{ Area\% } \\
\hline 1 & 53.933 & 212845 & 23254582 & 95.875 \\
\hline 2 & 61.524 & 12058 & 1000496 & 4.125 \\
\hline
\end{tabular}

Figure S176. HPLC record for chiral 5c 
$\mathrm{mAU}$

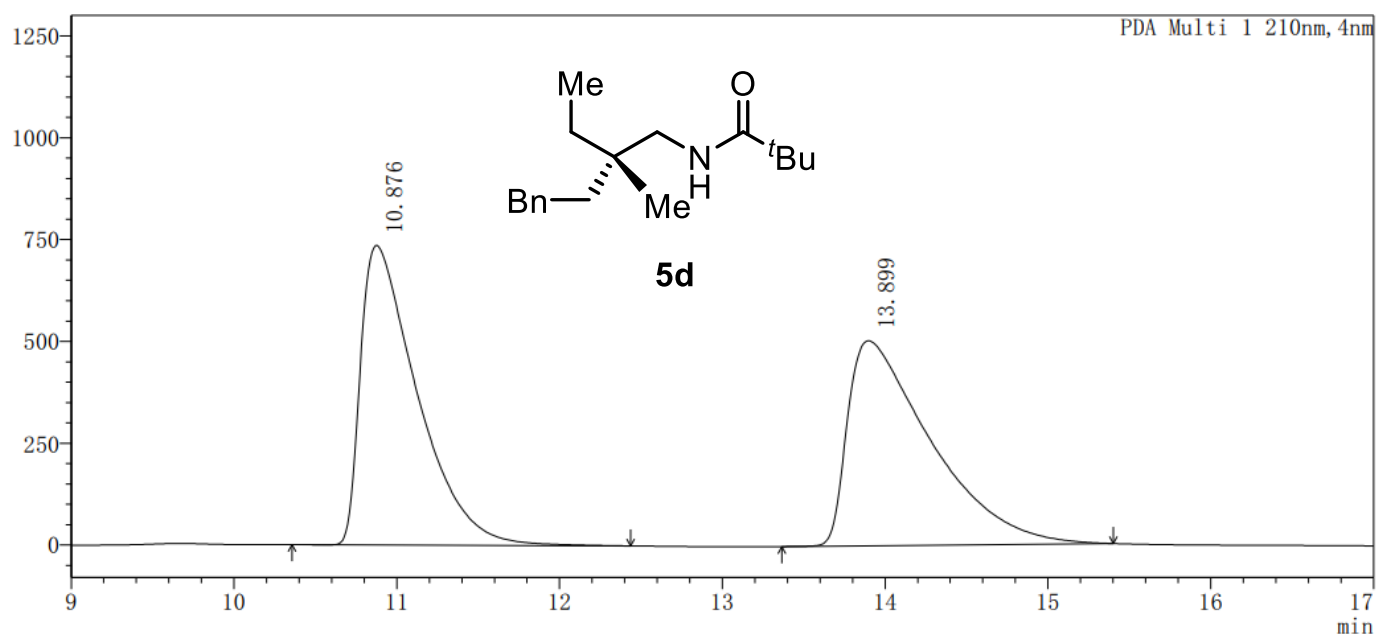

PDA Ch1 210nm

\begin{tabular}{|r|r|r|r|r|}
\hline Peak & Ret. Time & \multicolumn{1}{c|}{ Height } & \multicolumn{1}{c|}{ Area } & \multicolumn{1}{c|}{ Area\% } \\
\hline 1 & 10.876 & 735439 & 17835972 & 49.885 \\
\hline 2 & 13.899 & 503167 & 17918509 & 50.115 \\
\hline
\end{tabular}

Figure S177. HPLC record for racemic 5d

mAU

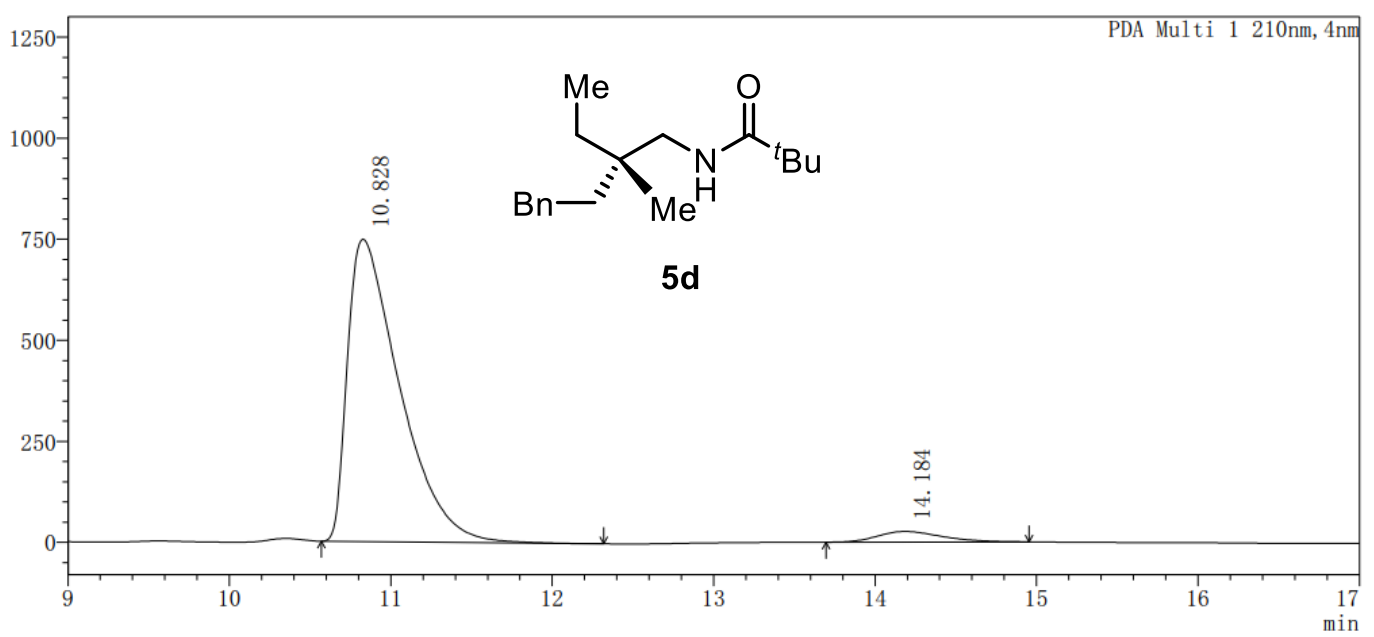

PDA Ch1 210nm

\begin{tabular}{|r|r|r|r|r|}
\hline Peak & Ret. Time & Height & \multicolumn{1}{c|}{ Area } & \multicolumn{1}{c|}{ Area\% } \\
\hline 1 & 10.828 & 748401 & 16829782 & 95.866 \\
\hline 2 & 14.184 & 26222 & 725817 & 4.134 \\
\hline
\end{tabular}

Figure S178. HPLC record for chiral 5d 


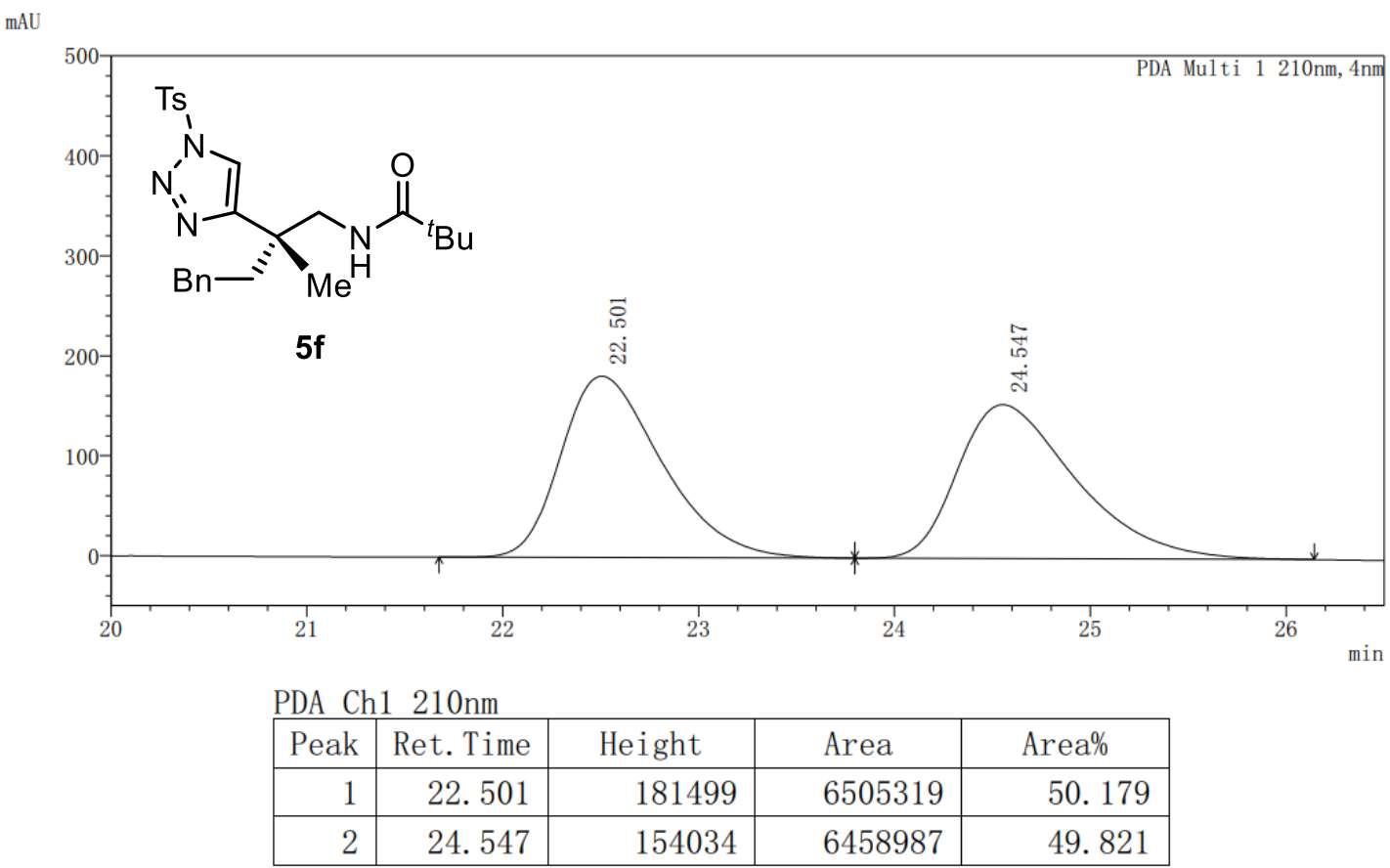

Figure S179. HPLC record for racemic $5 f$

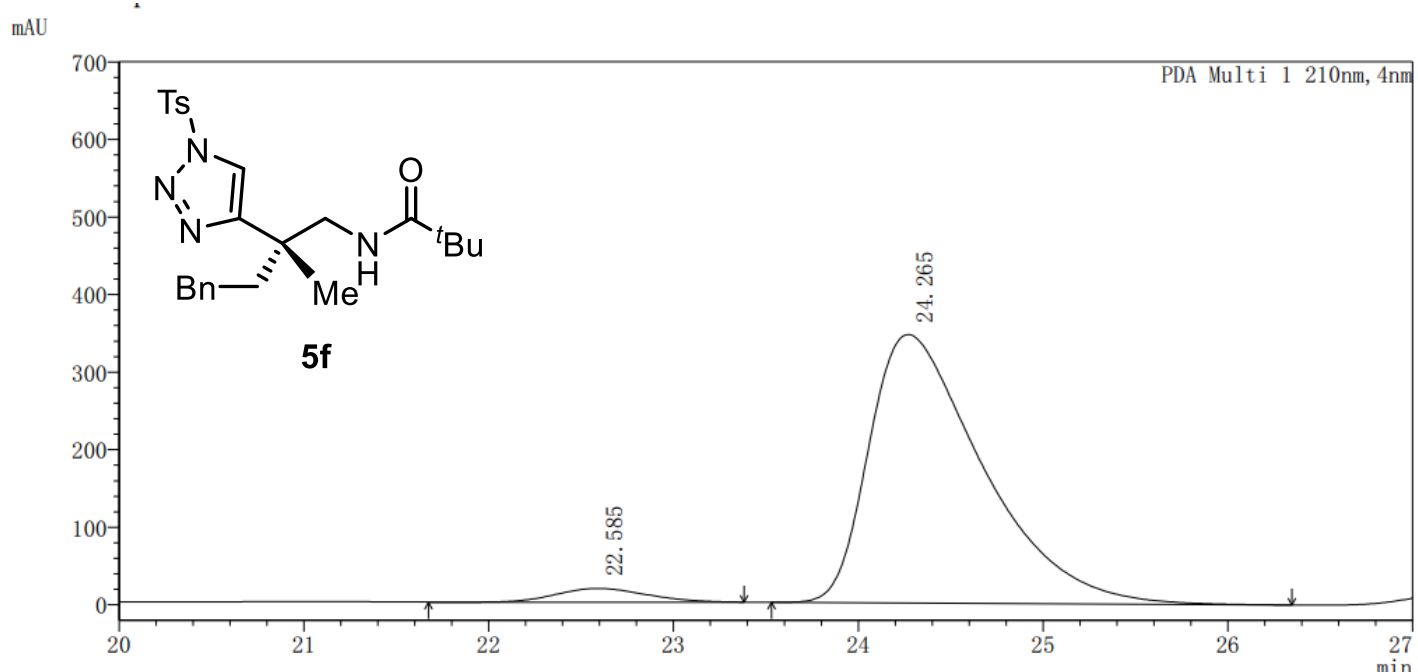

PDA Ch1 210nm
\begin{tabular}{|r|r|r|r|r|}
\hline Peak & Ret. Time & Height & \multicolumn{1}{c|}{ Area } & \multicolumn{1}{c|}{ Area $\%$} \\
\hline 1 & 22.585 & 17685 & 600825 & 3.893 \\
\hline 2 & 24.265 & 346182 & 14833592 & 96.107 \\
\hline
\end{tabular}

Figure S180. HPLC record for chiral $\mathbf{5 f}$ 
$\mathrm{mAU}$

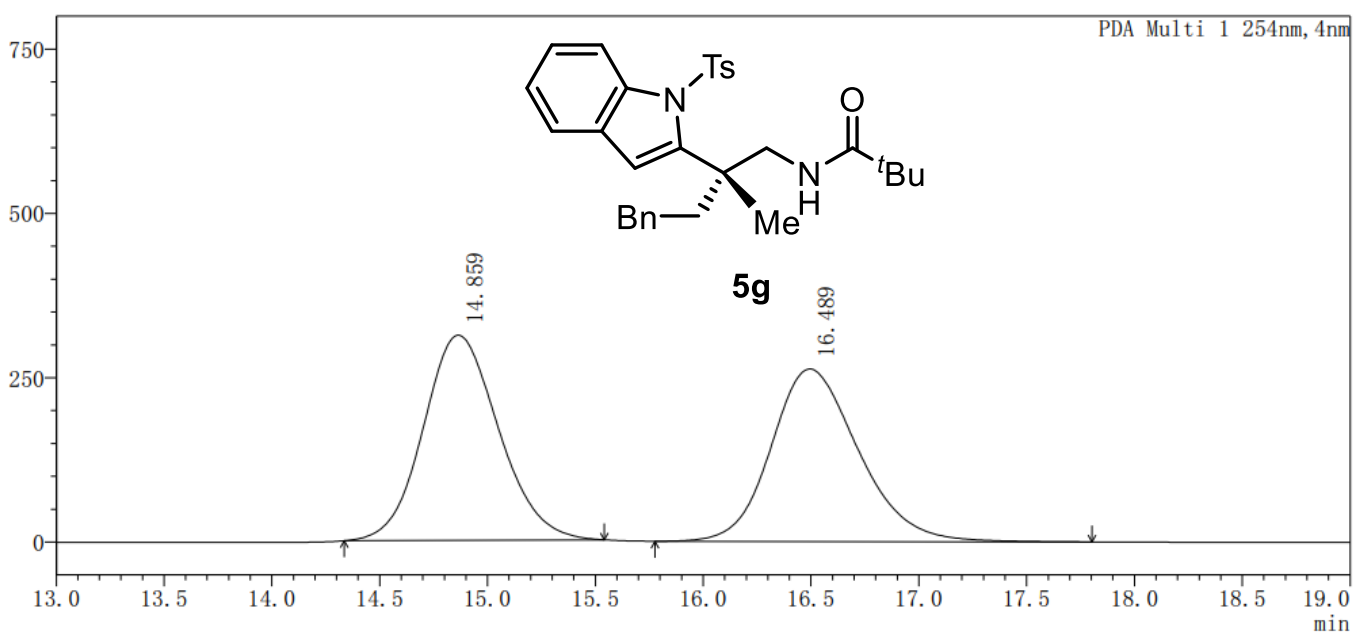

PDA Ch1 254nm

\begin{tabular}{|r|r|r|l|l|}
\hline Peak & Ret. Time & \multicolumn{1}{c|}{ Height } & \multicolumn{1}{l|}{ Area } & \multicolumn{1}{c|}{ Area\% } \\
\hline 1 & 14.859 & 312131 & 7603384 & 50.616 \\
\hline 2 & 16.489 & 262446 & 7418219 & 49.384 \\
\hline
\end{tabular}

Figure S181. HPLC record for racemic 5g

mAU

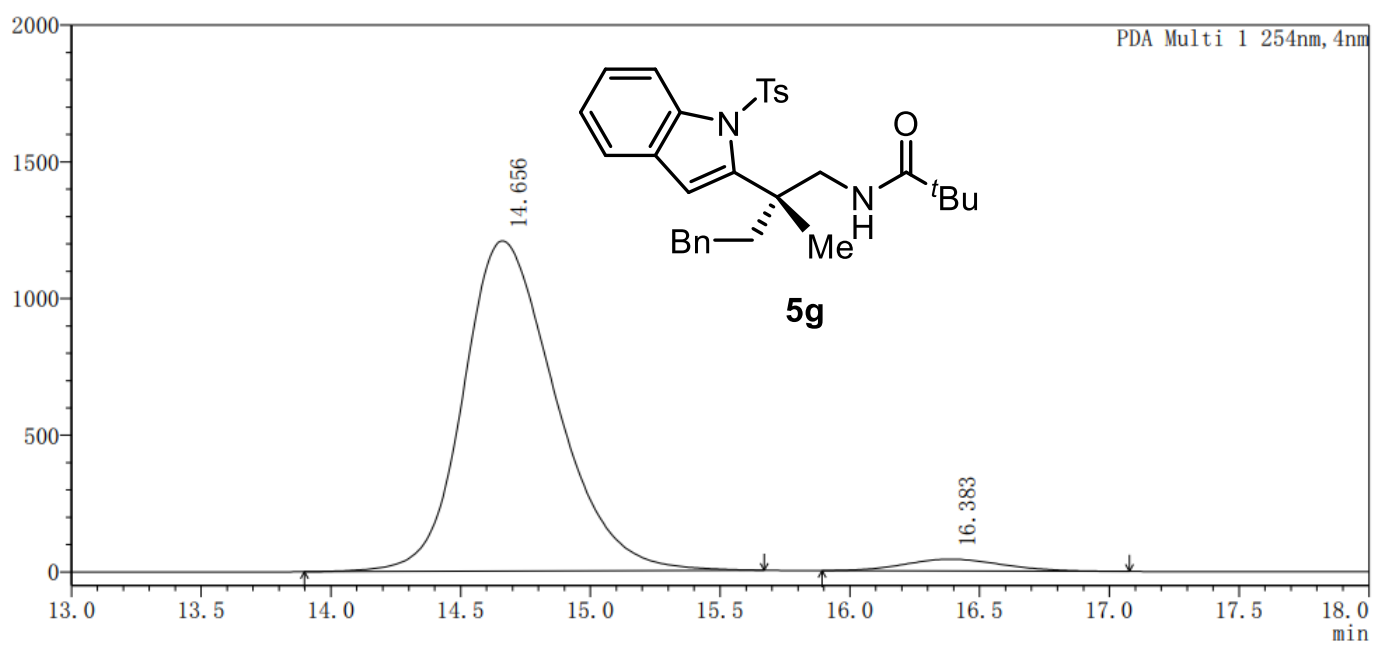

PDA Ch1 254nm

\begin{tabular}{|r|r|r|r|r|}
\hline Peak & Ret. Time & \multicolumn{1}{c|}{ Height } & \multicolumn{1}{c|}{ Area } & \multicolumn{1}{c|}{ Area\% } \\
\hline 1 & 14.656 & 1208022 & 29949998 & 96.367 \\
\hline 2 & 16.383 & 42922 & 1129068 & 3.633 \\
\hline
\end{tabular}

Figure S182. HPLC record for chiral 5g 
mAU

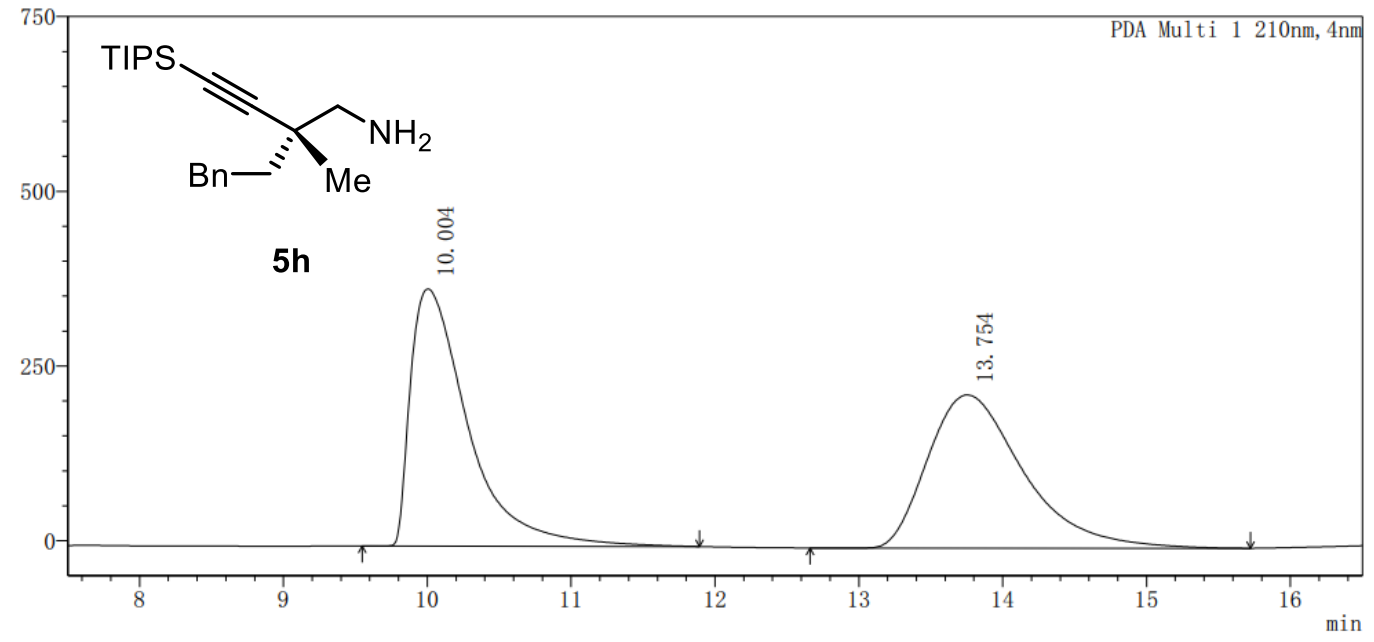

PDA Ch1 210nm

\begin{tabular}{|r|r|r|r|r|}
\hline Peak & Ret. Time & Height & \multicolumn{1}{c|}{ Area } & \multicolumn{1}{c|}{ Area\% } \\
\hline 1 & 10.004 & 368111 & 10239938 & 50.280 \\
\hline 2 & 13.754 & 219208 & 10125867 & 49.720 \\
\hline
\end{tabular}

Figure S183. HPLC record for racemic $5 h$

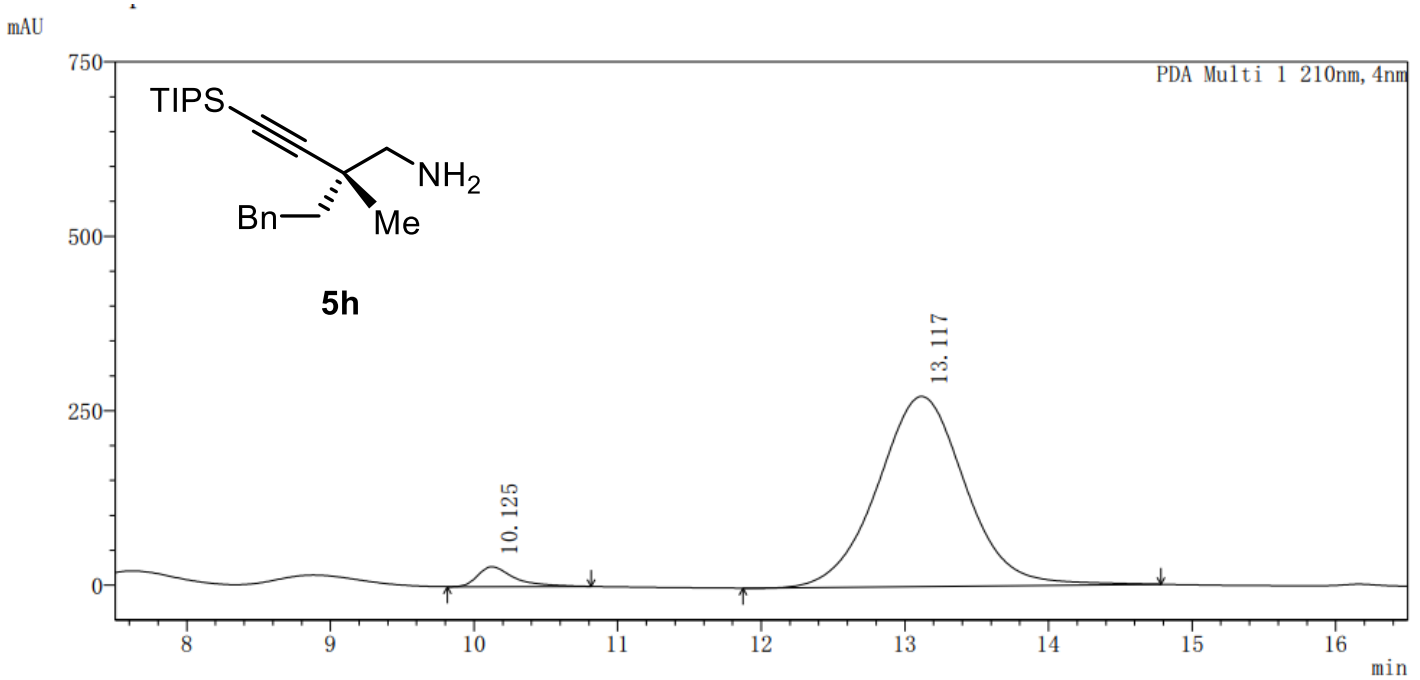

PDA Ch1 210nm

\begin{tabular}{|r|r|r|r|r|}
\hline Peak & Ret. Time & \multicolumn{1}{c|}{ Height } & \multicolumn{1}{c|}{ Area } & \multicolumn{1}{c|}{ Area\% } \\
\hline 1 & 10.125 & 28433 & 500789 & 4.136 \\
\hline 2 & 13.117 & 272401 & 11606010 & 95.864 \\
\hline
\end{tabular}

Figure S184. HPLC record for chiral $5 \mathrm{~h}$ 
mAU

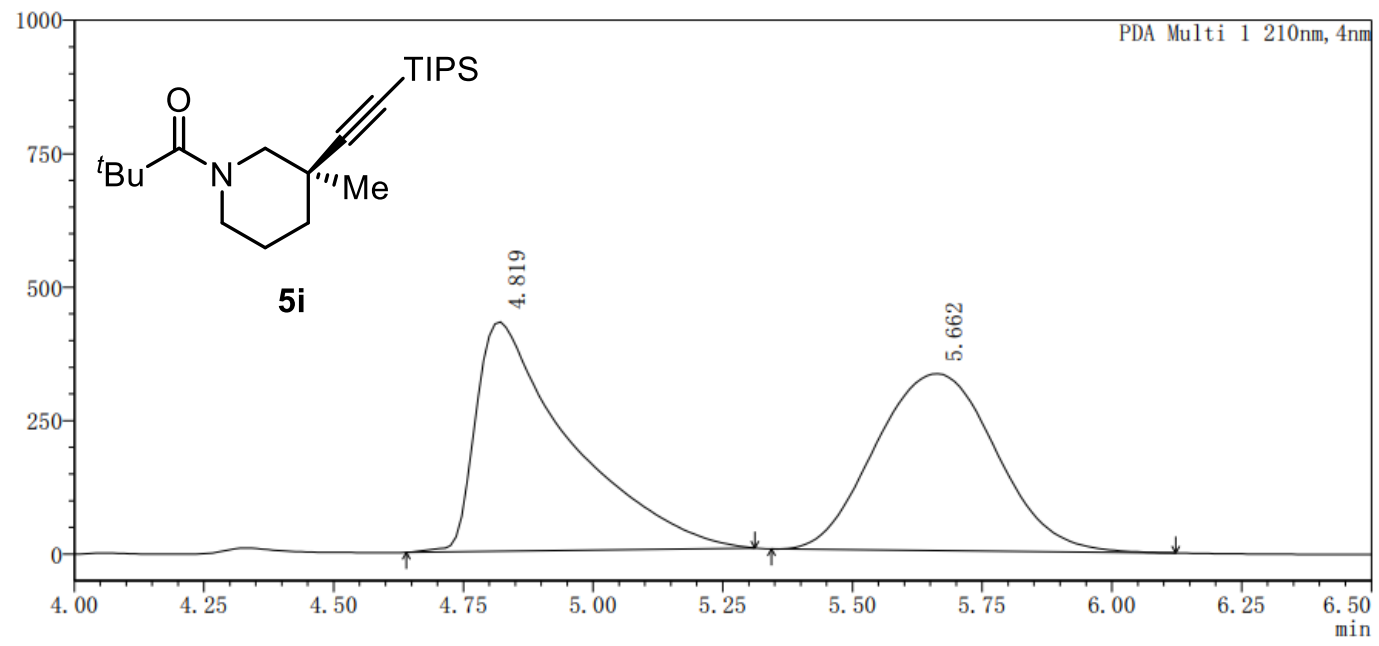

PDA Ch1 210nm

\begin{tabular}{|r|r|r|l|r|}
\hline Peak & Ret. Time & Height & \multicolumn{1}{|l|}{ Area } & \multicolumn{1}{c|}{ Area\% } \\
\hline 1 & 4.819 & 429586 & 5445016 & 50.323 \\
\hline 2 & 5.662 & 331663 & 5375085 & 49.677 \\
\hline
\end{tabular}

Figure S185. HPLC record for racemic 5i

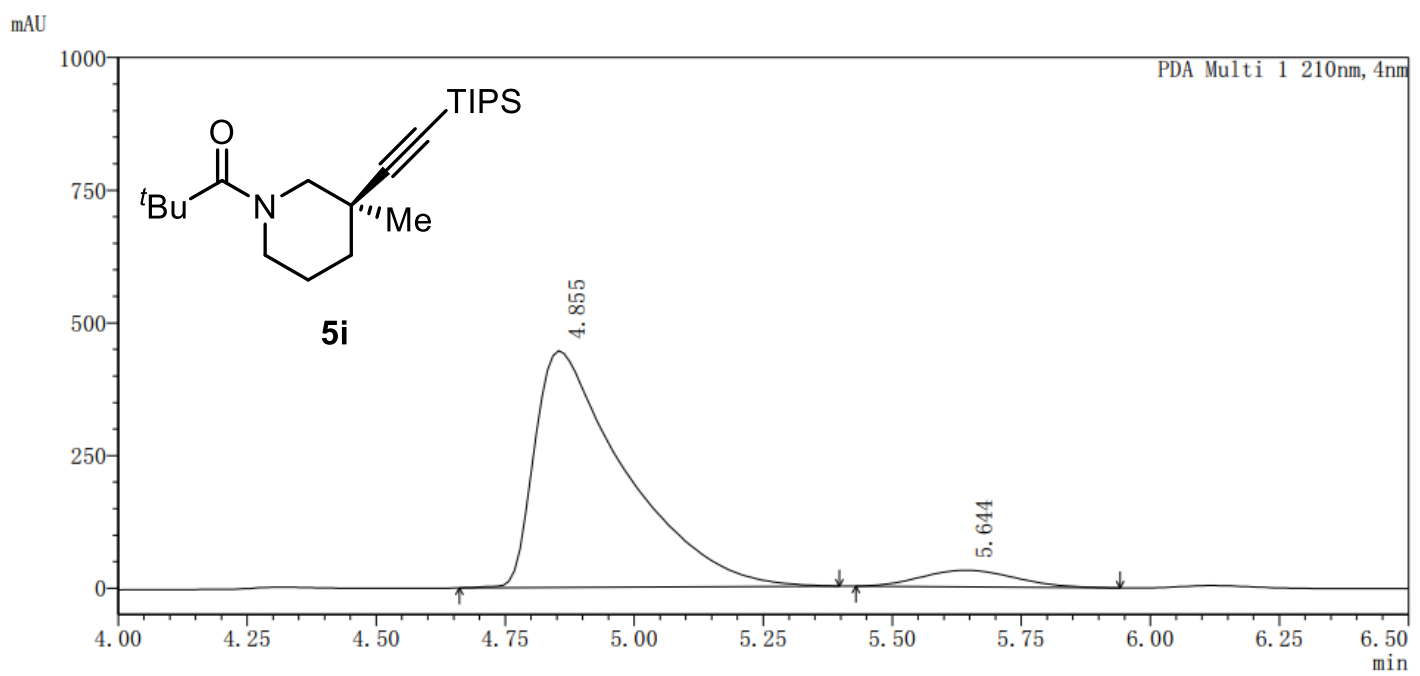

PDA Ch1 210nm

\begin{tabular}{|r|r|r|r|r|}
\hline Peak & Ret. Time & \multicolumn{1}{c|}{ Height } & \multicolumn{1}{c|}{ Area } & \multicolumn{1}{c|}{ Area\% } \\
\hline 1 & 4.855 & 445936 & 5458409 & 93.222 \\
\hline 2 & 5.644 & 31412 & 396850 & 6.778 \\
\hline
\end{tabular}

Figure S186. HPLC record for chiral 5i 